\title{
Catalog of Environmental Metrics for Hydropower
}

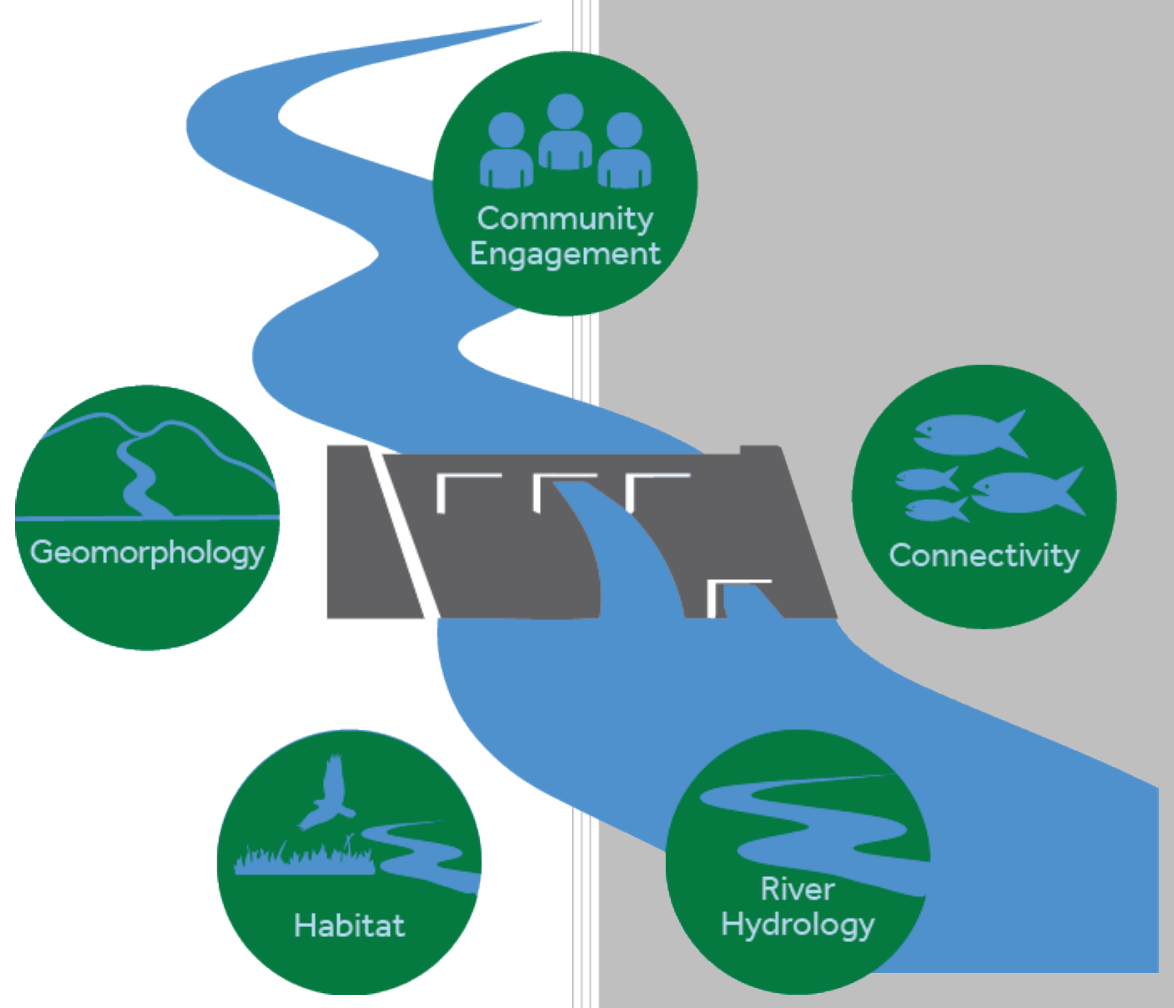

Curd, Shelaine

DeRolph, Chris McManamay, Ryan

Parish, Esther

Pracheil, Brenda

Smith, Brennan

June 2018

Approved for public release.

Distribution is unlimited 


\title{
DOCUMENT AVAILABILITY
}

Reports produced after January 1, 1996, are generally available free via US Department of Energy (DOE) SciTech Connect.

Website http://www.osti.gov/scitech/

Reports produced before January 1, 1996, may be purchased by members of the public from the following source:

\author{
National Technical Information Service \\ 5285 Port Royal Road \\ Springfield, VA 22161 \\ Telephone 703-605-6000 (1-800-553-6847) \\ TDD 703-487-4639 \\ Fax 703-605-6900 \\ E-mail info@ntis.gov \\ Website http://classic.ntis.gov/
}

Reports are available to DOE employees, DOE contractors, Energy Technology Data Exchange representatives, and International Nuclear Information System representatives from the following source:

Office of Scientific and Technical Information

PO Box 62

Oak Ridge, TN 37831

Telephone 865-576-8401

Fax 865-576-5728

E-mail reports@osti.gov

Website http://www.osti.gov/contact.html

This report was prepared as an account of work sponsored by an
agency of the United States Government. Neither the United States
Government nor any agency thereof, nor any of their employees, makes
any warranty, express or implied, or assumes any legal liability or
responsibility for the accuracy, completeness, or usefulness of any
information, apparatus, product, or process disclosed, or represents that
its use would not infringe privately owned rights. Reference herein to
any specific commercial product, process, or service by trade name,
trademark, manufacturer, or otherwise, does not necessarily constitute
or imply its endorsement, recommendation, or favoring by the United
States Government or any agency thereof. The views and opinions of
authors expressed herein do not necessarily state or reflect those of the
United States Government or any agency thereof.


ORNL Environmental Science Division

\title{
Catalog of Environmental Metrics for Hydropower
}

\author{
Curd, Shelaine \\ DeRolph, Chris \\ McManamay, Ryan \\ Parish, Esther \\ Pracheil, Brenda \\ Smith, Brennan
}

22 June 2018

Prepared by OAK RIDGE NATIONAL LABORATORY

Oak Ridge, TN 37831-6283

managed by

UT-BATTELLE, LLC

for the

US DEPARTMENT OF ENERGY

under contract DE-AC05-00OR22725 



\section{Contents}

\section{List of Figures}

\section{List of Tables}

1. Introduction

$1.1 \quad$ Origin \& Method / Proposal evolution

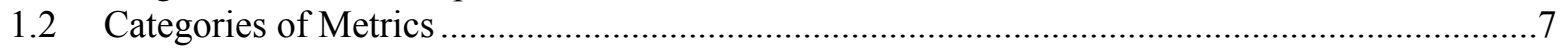

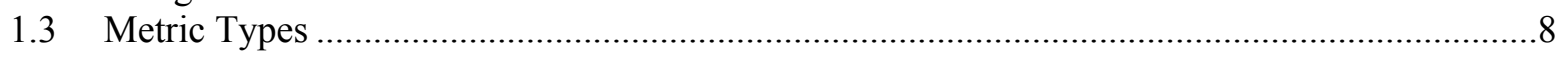

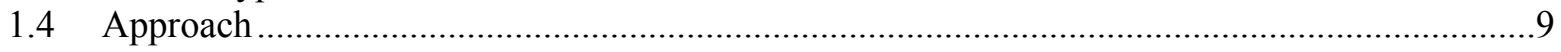

1.4.1 International Hydropower Association (IHA) Projects ...............................................

1.4.2 Low Impact Hydropower Institute (LIHI)/ Federal Energy Regulatory

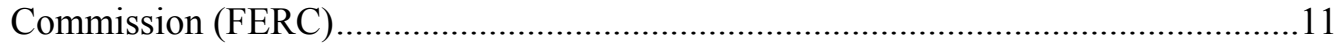

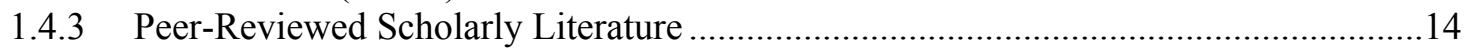

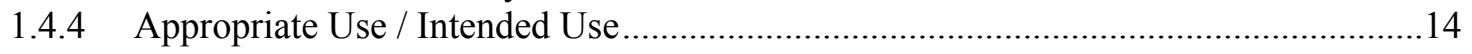

2. Other Types of Information Extracted

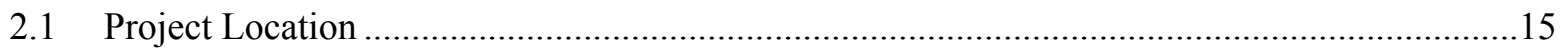

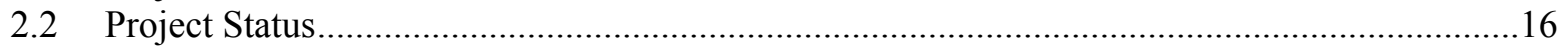

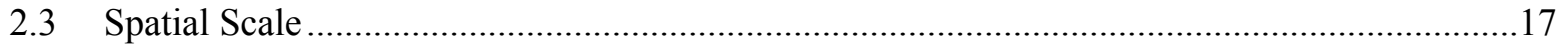

3. Resulting set of environmental metrics

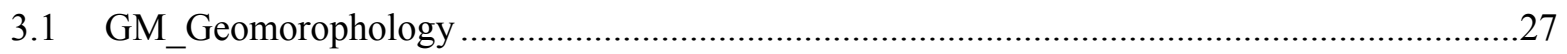

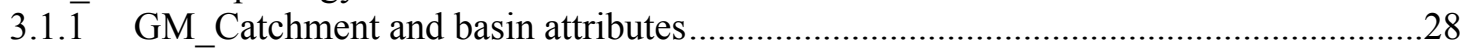

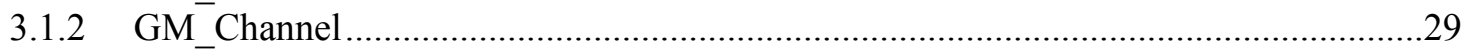

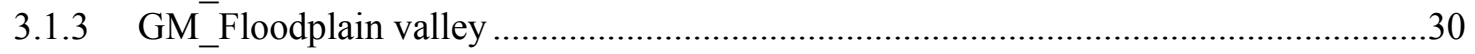

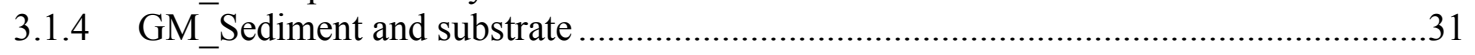

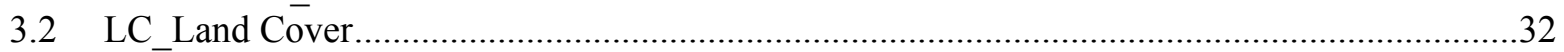

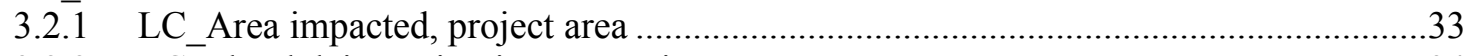

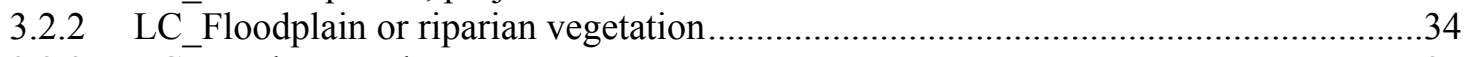

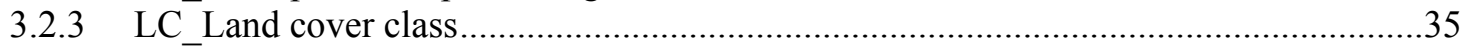

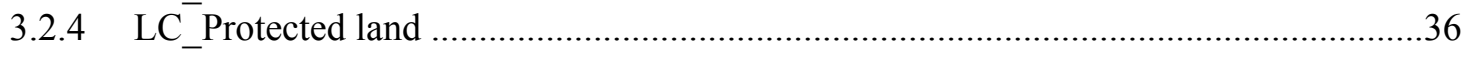

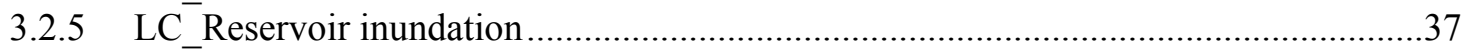

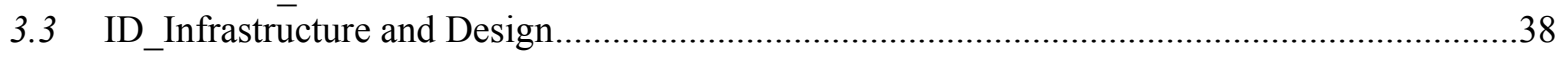

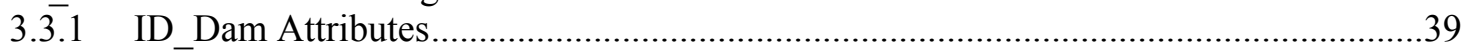

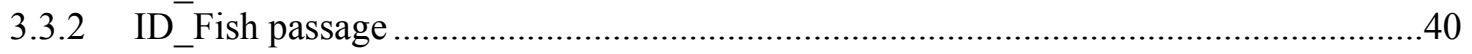

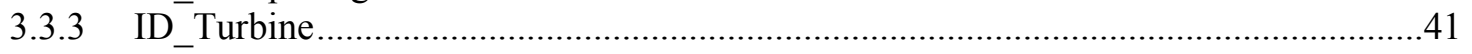

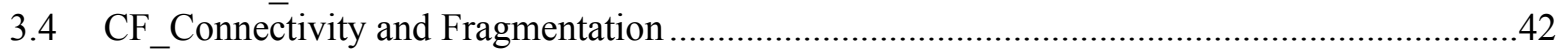

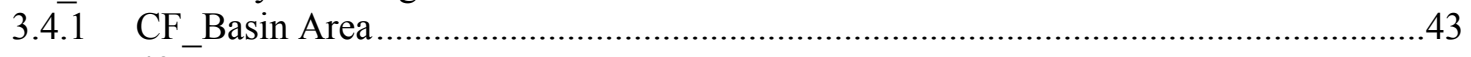

43

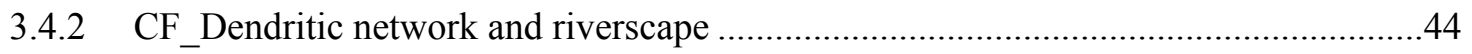

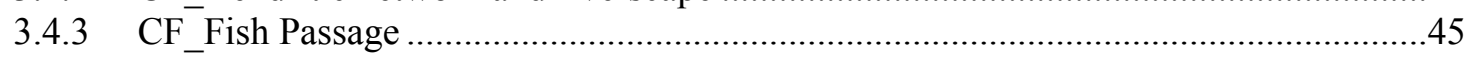

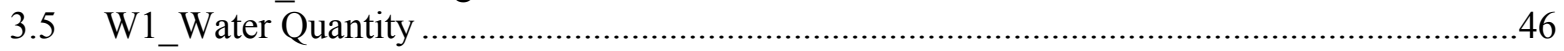

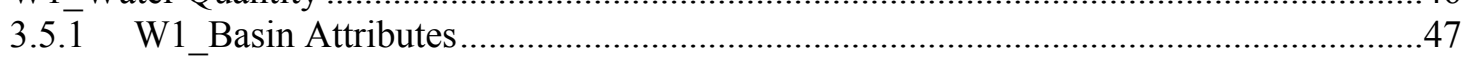

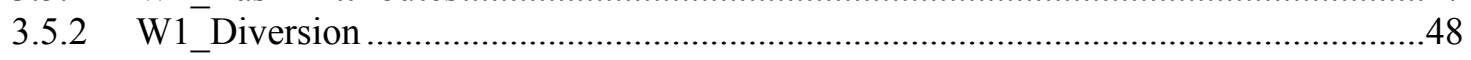

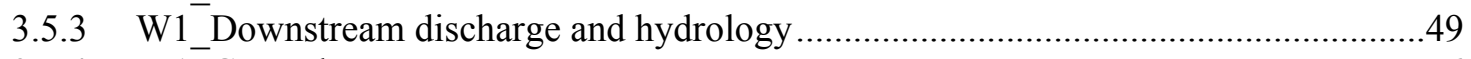

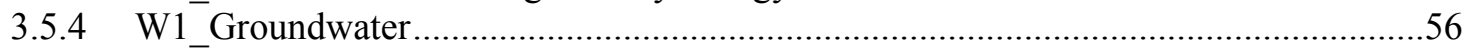

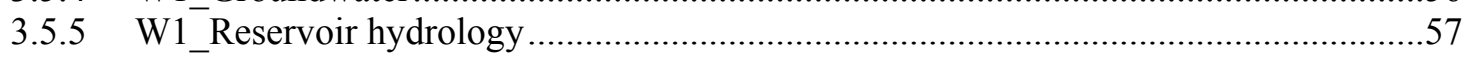




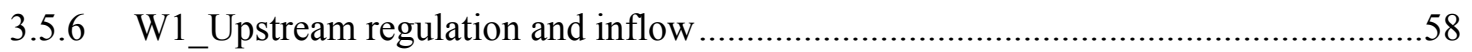

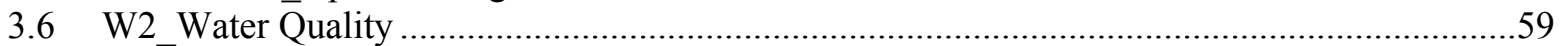

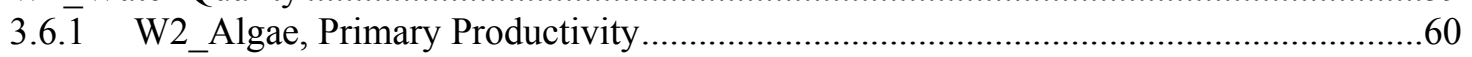

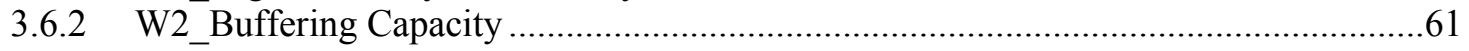

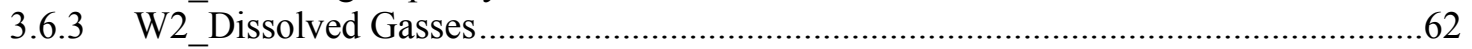

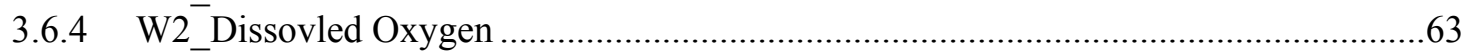

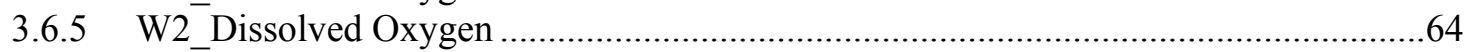

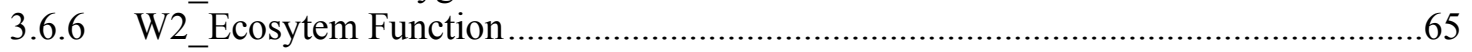

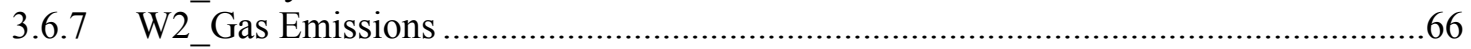

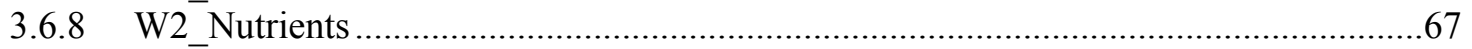

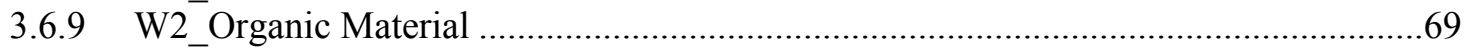

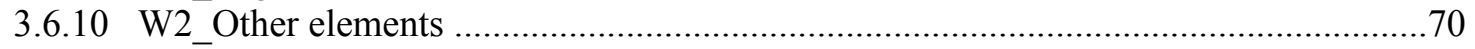

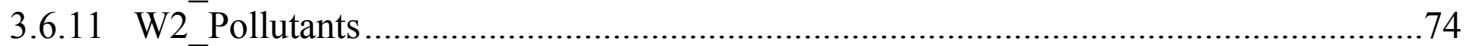

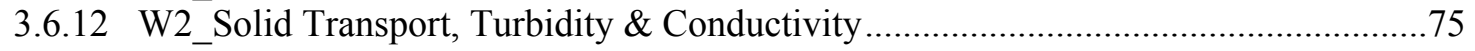

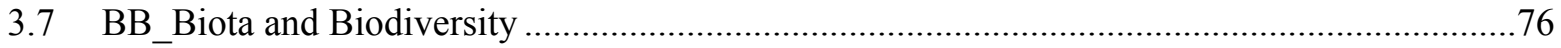

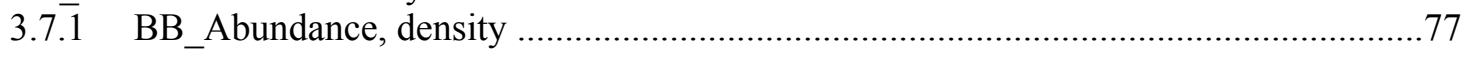

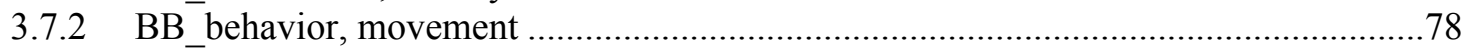

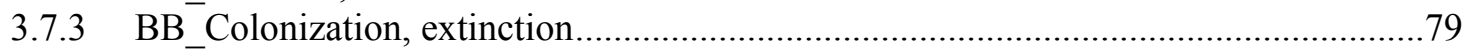

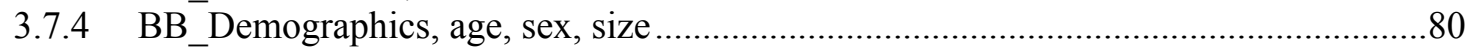

3.7.5 BB_Fitness, survival, growth, condition, reproduction, mortality .................................

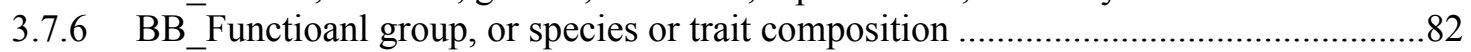

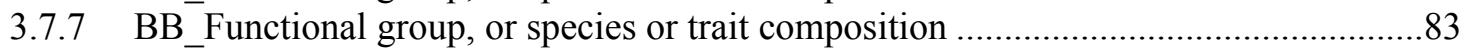

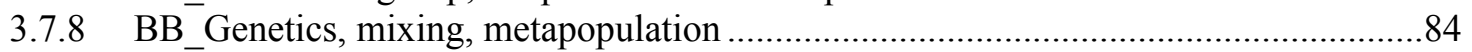

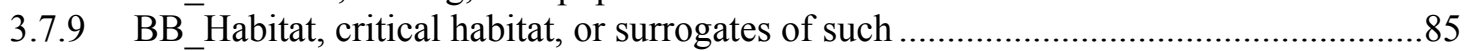

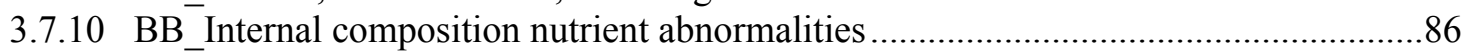

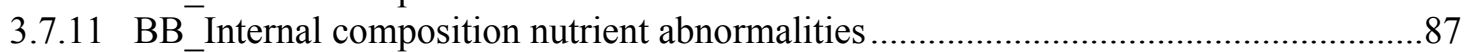

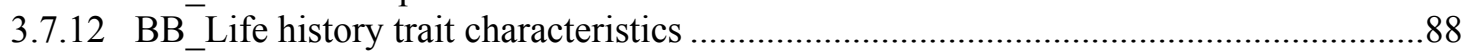

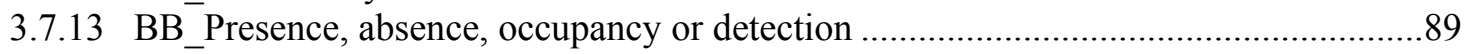

References

3.7.14 BB_Richness, diversity, evenness, or IBI types of measures ....................................90

AppendixA. Systematic review of peer-reviewed scholarly literature on hydropower metrics

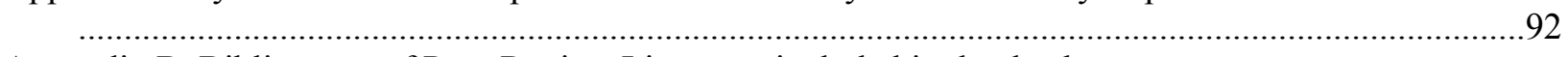

Appendix B. Bibliograpy of Peer-Review Literature included in the database

Appendix C. Summary of study locations and environmental metric categories extracted from the

97 peer-reviewed journal articles.

Appendix D. Bibliography of peer-review literature that passed the initial review filter but was not included in the database 


\section{LIST OF FIGURES}

Figure 1 - Environmental metrics relate to only a portion of the rigorous process of hydropower design and permitting, which also involves socioeconomic considerations...

Figure 2 - Representation of metric types: measure, statistic, and indicator

Figure 3- Raw metric extraction to Catalog

Figure 4 - Logo for IHA International Hydropower Sustainability Assessment Protocol

Figure 5 - International Hydropower Sustainability Assessment projects

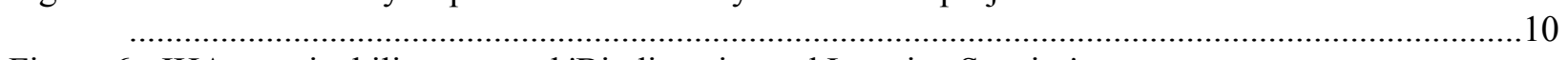

Figure 6 - IHA sustainability protocol 'Biodiversity and Invasive Species' scores

Figure 7. Locations of non-Federal U.S. Hydropower projects certified by LIHI and recently

Figure 8 - Systematic Review of Scholarly Peer-Reviewed Literature

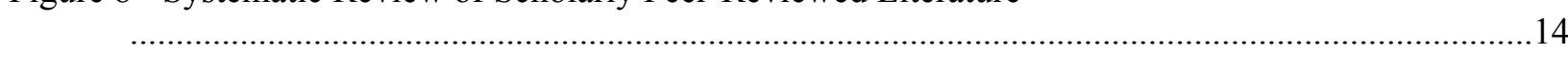

Figure 9 - Map showing the 231 study locations used to collect the metrics recorded for this analysis of the environmental effects of hydropower.................................................................

Figure 10 - The represented phases of hydropower development associated with the extracted

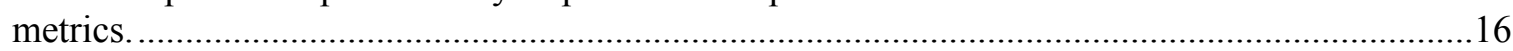

Figure 11 - Spatial scale associated with catalogued metrics.

Figure 12 - Size and ownership distribution of U.S. hydropower projects captured by this environmental metrics for hydropower literature review (left) relative to the entire U.S. hydropower fleet (right).

Figure 13 - Summary of captured environmental metrics by category, sector and type.

Figure 14- Distribution of the environmental metrics by category and document type

Figure 15- Geographic distribution of the collected environmental metrics by continent (top) and by U.S. region (bottom). .

\section{LIST OF TABLES}

Table 1 - List of IHA hydropower projects reviewed for environmental metrics

Table 2. List of five FERC/LIHI projects reviewed for environmental metrics

Table 3 Spatial scales of environmental metrics related to hydropower 
Table 4 Emergent sub-categories (45) of the environmental metrics (3183) collected through this literature review of 117 documents. Metrics totals are also shown by type: $M=$ Measures, $\mathrm{S}=$ Statistics, and $\mathrm{I}=$ Indicators. 


\section{INTRODUCTION}

\subsection{ORIGIN \& METHOD / PROPOSAL EVOLUTION}

The catalog evolved as a project from discussions on hydropower new stream reach development and sustainability. While sustainability includes balancing economic, social and environmental aspects, this catalog focuses on environmental metrics used in assessing the environmental effects of new hydropower development. Environmental effects in this context refers to the effects on the physical and ecological functions of streams and biota. This catalog of metrics, and metric methodology will enable stakeholders to define, assess, and communicate their own concepts of environmental sustainability with efficacy, clarity, and transparency.

Cataloging environmental metrics should not be confused with the outcomes of existing regulatory processes since those Acts stipulate what agencies must do, must not do, and may choose to do based upon a thorough assessment of the project's potential impacts and developed protection, mitigation and enhancement measures. Environmental metrics, on the other hand, represent the most fundamental levels of environmental information, upon which the procedural stipulations mentioned above are based. Examining the raw information underlying the basis of existing protocols, certifications, regulations, and their outcomes is a level of transparency needed to determine the current state of practice and the adequacy of those processes. Environmental metrics also enable stakeholder input into regulatory decision-making that often results in trade-offs. This Environmental Metrics for Hydropower project will translate science findings into useful metrics, thereby enabling stakeholders and agencies to be more efficient, concise, transparent, and collaborative in expressing their priorities and concerns about hydropower development.

The overall long-term intent of the project is to identify a measurable, repeatable, and broadly understandable suite of metrics for quantifying the environmental effects of hydropower. This project will also document the variability of metrics used to evaluate environmental impact. The importance of these metrics is to help identify and quantify the benefits and cost options during project development.

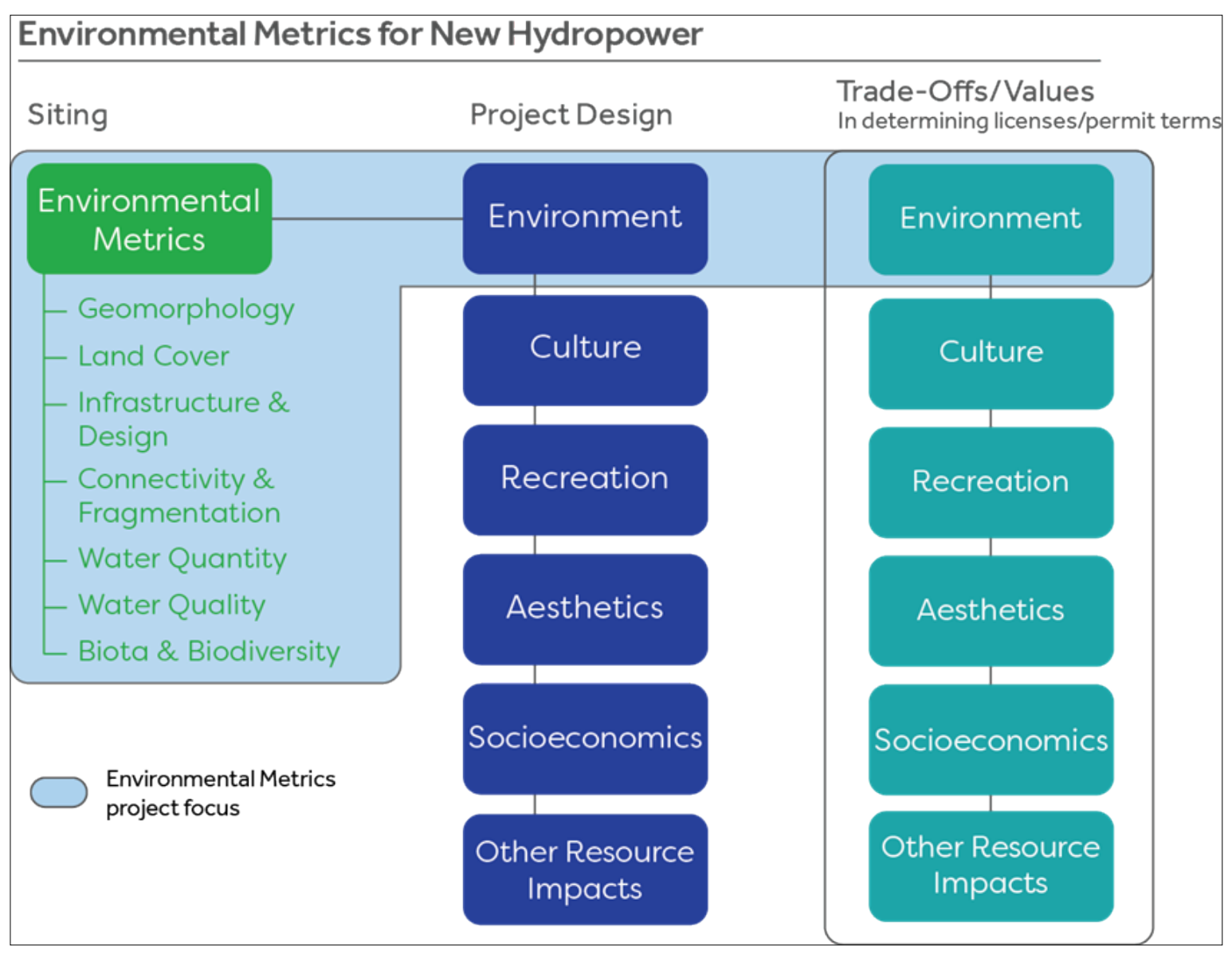

Figure 1 - Environmental metrics relate to only a portion of the rigorous process of hydropower design and permitting, which also involves socioeconomic considerations.

ORNL Catalog of Environmental Metrics for Hydropower - 7 
Therefore, these metrics must have measurable units that are agreed upon and can be consistently applied across the U.S.

\subsection{CATEGORIES OF METRICS}

In the development of standard communications avenue for this study a shared terminology document (Pracheil et al. 2016) was developed that is more than a simple glossary. To capture the general environmental concepts that govern river ecology, enable thematic analysis and consistent visualization of findings, the Environmental Metrics for Hydropower (EMH) project classifies information into 7 categories which are applicable across siting, project design and permitting (Figure 1). These 7 categories are Geomorphology, Land Cover, Infrastructure and Design, Connectivity and Fragmentation, Water Quantity, Water Quality and Biota and Biodiversity. As metrics were identified, they were assigned one category.

1. Geomorphology -Geomorphology is the dynamic evolution of topographic and bathymetric features created within an ecosystem. Hydropower development can disrupt a river system's geomorphologic equilibrium through altered sediment and flow regimes. These changes have the potential to impact the availability and quality of habitat for plants and animals within the system.

2. Land cover - Land cover type (the physical material on the earth's surface) is an important measure of ecosystem health because it influences many other environmental properties ranging from river and floodplain sedimentation rates to fragmentation of habitats and wildlife populations at scales ranging from site to landscape. Land cover changes can be used to more-fully describe ecosystem changes associated with hydropower development, such as increases in wetted surface from reservoir formation, and fragmentation of the surrounding landscape through installation of supporting infrastructure (e.g., transmission lines, roads). Land cover characterization pre- and post-hydropower development is an important component of understanding hydropoweraffected ecosystems.

3. Infrastructure and Design-Hydropower infrastructure involves construction of structures in-stream (for impounding water and generating power) and in adjacent riparian and terrestrial lands (for transmitting power and accessing the site). The selection of hydropower equipment, associated infrastructure and management practices can bear directly and indirectly on the other six Science Core Concepts through a variety of factors such as increased land cover fragmentation for running transmission lines, exposure of animals and humans to electromagnetic fields, changes in the volume and timing of water releases, and the use of industrial lubricants needed to keep hydropower turbines properly working.

4. Connectivity and Fragmentation - Ecosystem connectivity (the degree to which a land cover type or ecosystem maintains continuity) and fragmentation (the degree to which an ecosystem or land cover type is disconnected) can affect the habitat quantity and quality for organisms in an ecosystem. Dams and their associated infrastructure can disrupt aquatic, riparian, and terrestrial connectivity, as well as groundwater connectivity, all of which can directly impact biota. Quantifying connectivity changes is therefore important for a full accounting of the environmental effects of hydropower.

5. Water Quantity -Water quantity refers to the amount of water found in-stream, in a reservoir or in groundwater stores and includes flow magnitude, duration, frequency, timing, and rate-of-change of flows. Hydropower development can alter the quantity of water in several ways through water storage in reservoirs, increased evaporation rates, change availability of in-channel water downstream of a dam, as well as change the quantity of ground water both upstream and downstream of dams. Because hydropower systems may be operated to fill a variety of purposes, changes to water quantity may occur at a variety of temporal scales ranging from diel to annual. Changes to water quantity can ultimately affect human and wildlife populations through altered water supplies for a variety of end uses (e.g., agricultural, drinking, municipal, industrial, recreational) and through changes in habitat availability.

6. Water Quality - Water quality characteristics such as temperature and concentrations of dissolved oxygen, velocity, nutrients and contaminants can be directly or indirectly affected by hydropower development and operation. Changes in water quality can adversely affect the health of humans and wildlife.

7. Biota and biodiversity - The types of plant and animal species found in a watershed, as well as their absolute abundance and relative abundance to each other, reflect the overall health of an ecosystem. Shifts in aquatic, riparian and terrestrial populations and communities have been linked to several aspects of hydropower construction and operation, including decreased longitudinal connectivity and changes in flow velocities in rivers, inundation of uplands

ORNL Catalog of Environmental Metrics for Hydropower - 8 
upstream of dams, changes in ground water depth both up and downstream of dams, and changes in sediment and flow regimes. Identifying metrics to accurately assess population and community changes are essential to understanding the environmental effects of hydropower.

\subsection{METRIC TYPES}

It is recognized that different metrics require different levels of analysis and interpretation. Throughout this study metrics are assigned a type that best describes this level of analysis. These are broken into three types, measurement, statistic and indicator.

Additional attributes were assigned including the type of metric:

- Measure - a direct measurement of environmental phenomenon (e.g., temperature readings, species counts)

- Statistic - a mathematical summarization of collected environmental measures (e.g., hydrograph)

- Indicator - a measure or statistic whose values have been used to indicate positive or negative movement toward or away from a goal established by stakeholders (e.g., reforestation)

A measurement with a threshold should be a red flag to look for additional information/rationale behind it (i.e., we need to know the rationale taken to raise the measurement to the level of an indicator).

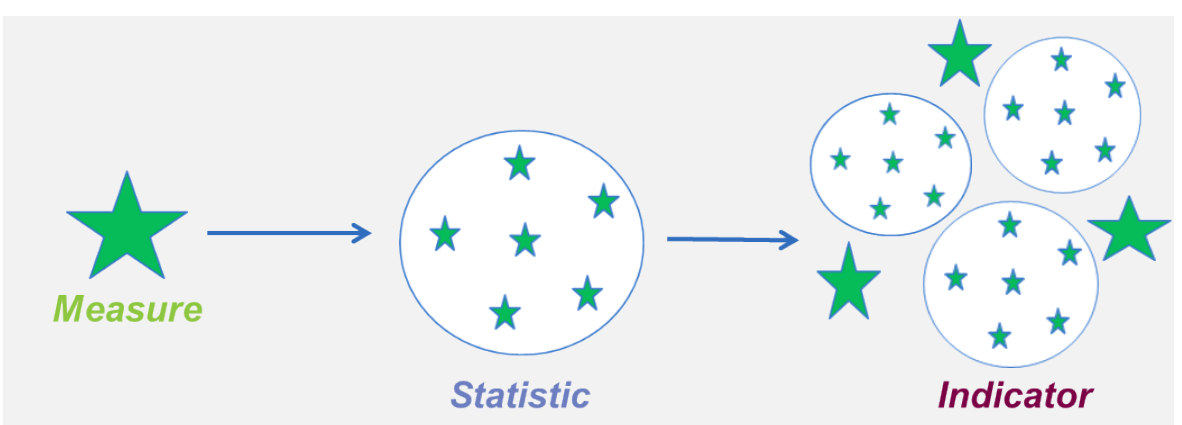

Figure 2 - Representation of metric types: measure, statistic, and indicator 


\subsection{APPROACH}

Documenting the metrics used to describe the environmental effects of hydropower is a difficult task, as the 'environment' includes aquatic and terrestrial ecosystems and spans multiple disciplines of environmental and ecological sciences. Executing this task must start at a very broad level, principally coming to a common understanding of what the "environment" includes and a common terminology of how we go about describing it. Hence, an organizational framework is required to catalog environmental metrics across the many dimensions of the environmental science space. In developing the framework for the metric extraction, ORNL developed a working document titled Environmental Metrics for New Hydropower: Terminology and Categories (Pracheil, et. al. 2016) which is more than a simple glossary. It specifies and categorizes environmental metrics (Section I), defines relevant environmental terminology (Section II), and defines general ecological concepts (Section III) needed to foster clear communication about environmental issues pertaining to hydropower development and operation.

Environmental metrics were extracted from multiple sources: (1) International Hydropower Association (IHA) Hydropower Sustainability Assessment Protocol (HSAP) (2) Low Impact Hydropower Institute (LIHI) (3) Federal Energy Regulatory Commission (FERC) and (4) Systematic review of the peer-reviewed scholarly literature using guidelines established by the Center for Environmental Evidence (CEE 2013) The environmental metrics used by hydropower certificatory bodies (LIHI, IHA) and the current U.S hydropower regulatory domain (FERC licenses) including their satisfaction of other pieces of the current hydropower regulation such as National Environmental Policy Act (NEPA) and Council on Environmental Quality (CEQ), which require several metrics to be evaluated. Because the dimensions of environmental information can be described in multiple levels of detail, a hierarchical template was developed to organize the catalog of metrics and their attributes. This included the development of 7 main environmental categories, which are divided into 45 more specific parameter categories, in addition to a number of attributes describing the hydropower project and systems under consideration for each metric. Additionally, a template was devised to describe the nature of how metrics convey information, such as in raw measurements, statistical summarization, or in the form of indicators that inherently contain heuristic values.

The process of extracting metrics for each of these sectors is provided in more detail below.

\subsubsection{International Hydropower Association (IHA) Projects}

Environmental metrics were captured from a subset of 13 hydropower Hydropower
Sustainability Assessment Protocol

Figure 4 - Logo for IHA International Hydropower Sustainability Assessment Protocol projects that have been reviewed and published using the International Hydropower Association's (IHA's) Hydropower Sustainability Assessment Protocol (HSAP). All 13 published IHA HSAP projects were reviewed for sustainability scores and gaps, dam locations were determined using Google Earth, and a crosswalk chart of definitions was developed to facilitate understanding of the different sustainability topics which apply at each of the four stages of HSAP application (i.e., Early stage, Preparation stage, Implementation stage, or Operation stage).

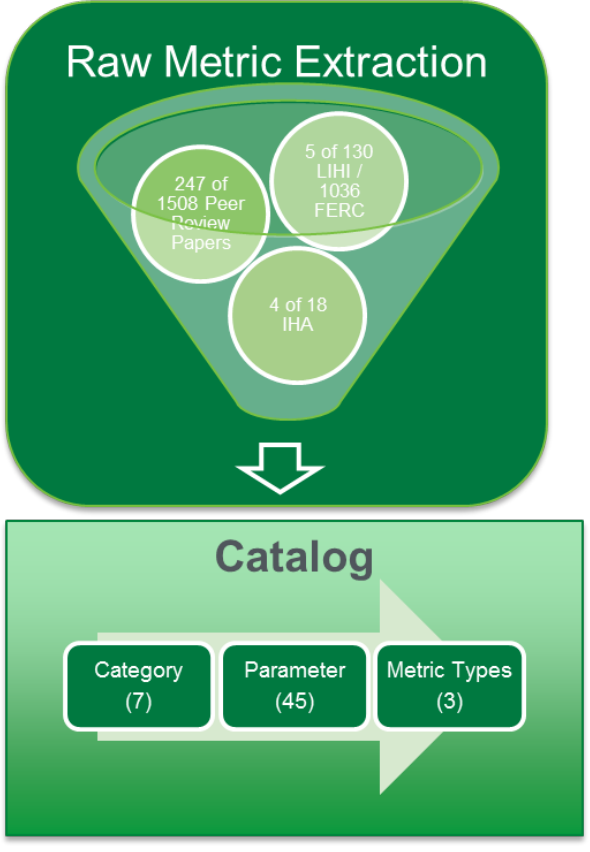

Figure 3- Raw metric extraction to Catalog 
An initial subset of IHA projects was selected to span the globe, as shown in Figure 5. Table 1 lists details of the 4 IHA projects selected for detailed environmental metrics extraction.

Table 1 - List of IHA hydropower projects reviewed for environmental metrics

\begin{tabular}{lllllll}
\hline $\begin{array}{l}\text { Hydropower } \\
\text { Project }\end{array}$ & Country & River & Owner & $\begin{array}{l}\text { Capacity } \\
\text { (MW) }\end{array}$ & $\begin{array}{l}\text { IHA HSAP } \\
\text { Protocol Stage }\end{array}$ & Metrics \\
\hline Chaglla & Peru & Huallaga & $\begin{array}{l}\text { Empresa de } \\
\text { Generación } \\
\text { Huallaga S.A. }\end{array}$ & 456 & Implementation & 43 \\
Kabeli A & Nepal & Kabeli & $\begin{array}{l}\text { Kabeli Energy } \\
\text { Limited }\end{array}$ & 37.6 & Preparation & 40 \\
Walchensee- & Germany & Isar & E.ON Hydro Fleet & 124 & Operation & 8 \\
$\begin{array}{l}\text { kraftverk } \\
\text { Trevallyn }\end{array}$ & Australia & Esk & Hydro Tasmania & 96 & Operation & 16 \\
\hline
\end{tabular}

Three elements to consider in an IHA Hydropower Sustainability Assessment:

1. "The graded performance within each sustainability topic provides the opportunity to promote structured continuous improvement." (HSAP Nov2010)

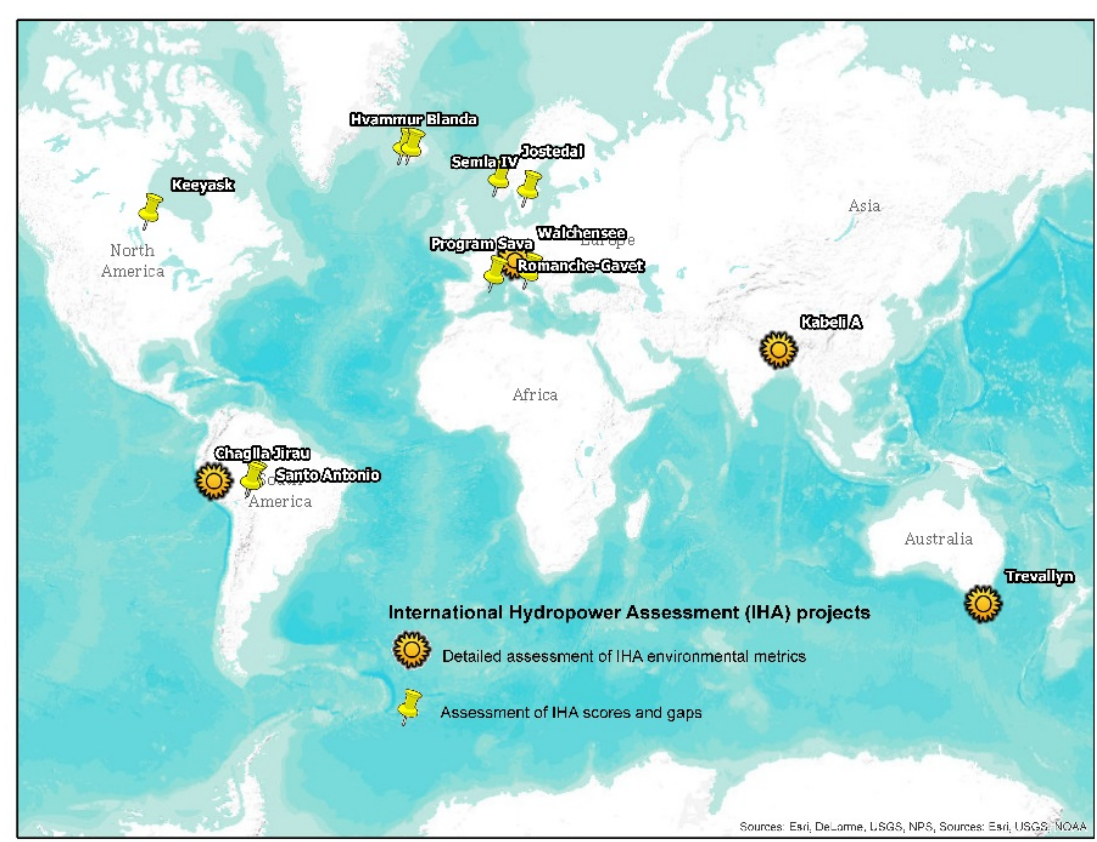

Figure 5 - International Hydropower Sustainability Assessment projects

2. "The HSAP should be applied in a collaborative way, to ensure the best availability of information and points of view. The development and evaluation of a hydropower project will involve many actors with different roles and responsibilities. It is recognized that both development and operation may involve public entities, private companies or combined partnerships, and responsibilities may change as the project progresses through its life cycle." (HSAP Nov2010)

3. "The terms and conditions define an official assessment as one which: (1) Is carried out by an independent accredited assessor; (2) Involves the principal organisation responsible for the project [at the current stage], demonstrated by their written support; and (3) Meets any other guidelines for official assessment published by the Hydropower Sustainability Assessment Council." (HSAP Nov2010)

Each HSAP sustainability score reflects the graded performance of the project from lowest level (1) to highest level (5) relative to good and best practices across the industry. The recorded scores and gaps are meant to provide the opportunity to promote structured continuous improvement and are not intended for pass/fail determination. Figure 6 provides an example of how all of the published IHA

ORNL Catalog of Environmental $\mathrm{N}$

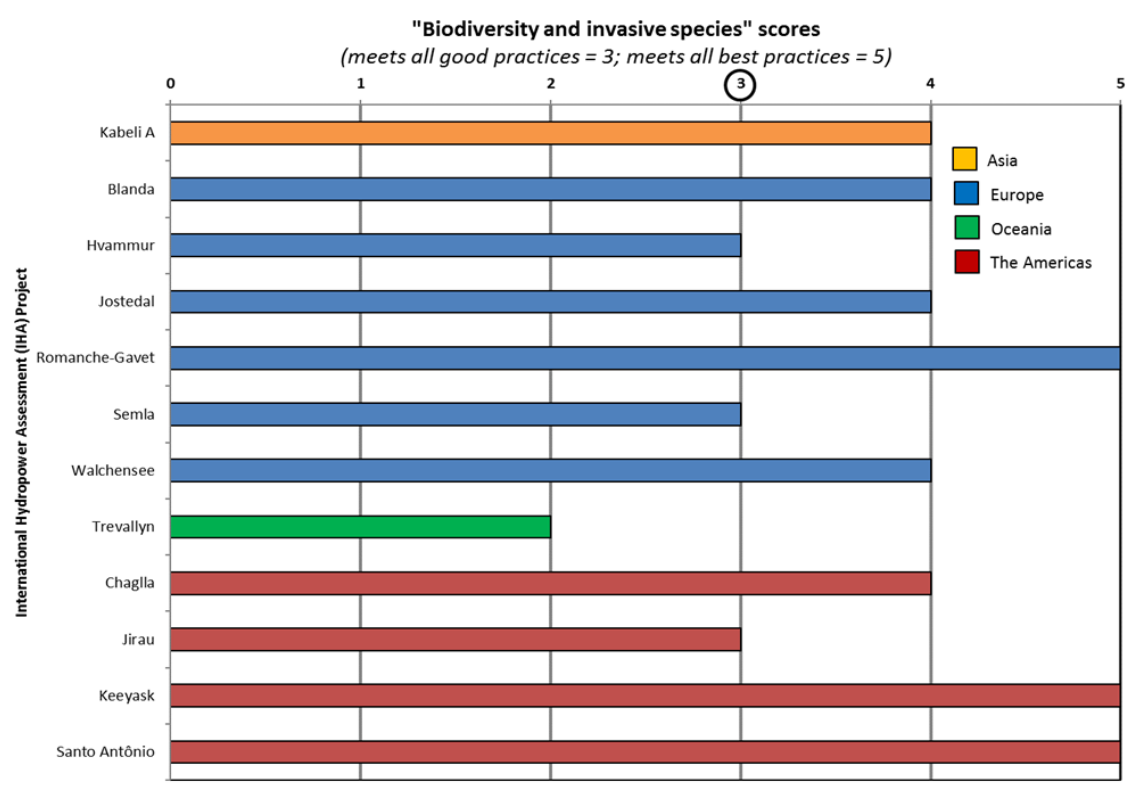

Figure 6 - IHA sustainability protocol 'Biodiversity and Invasive Species' scores 
projects scored with regard to the 'Biodiversity and invasive species' sustainability topic. For this topic, only one project (Trevallyn) showed a significant gap relative to good practices. Three projects (Romanche-Gavet, Keeyask, and Santo Antônio) were found to meet the best possible practices with regard to biodiversity.

Disentangling environmental metrics from socioeconomic metrics proved difficult due to IHA's integrated evaluation approach.

Environmental metrics were found to be closely associated with these HSAP sustainability topic areas:

- Biodiversity and invasive species

- Downstream flow regimes

- Erosion and sedimentation

- Reservoir planning

- Waste, noise and air quality

- Water quality

A total of 100 environmental metrics corresponding to all of the 7 EMH Categories were gleaned from the analysis of the four 4 IHA projects selected for detailed analysis. An additional 7 metrics not pertaining to the EMH categories were also captured in the metrics database for future consideration. These 7 metrics related to air quality, noise, solid waste generation, soil contamination and electromagnetism.

\subsubsection{Low Impact Hydropower Institute (LIHI)/ Federal Energy Regulatory Commission (FERC)}

In order to provide a sub-sample of environmental metrics applied within LIHI protocols and FERC regulations, five U.S. non-federal hydropower projects were chosen that were both (a) LIHI certified, and (b) have recently undergone FERC relicensing (Figure 7). Projects were selected to represent different geographical regions of the U.S. and a diversity in project sizes (megawatt capacity and infrastructure). Selected projects and their characteristics are listed in Table 1.

For each project, all LIHI documentation was openly available through the institute's webpage and reviewed. The structure of the LIHI certification process is

\section{LIHI impact criteria}

- Ecological Flow Regimes

- Water Quality Protection

- Upstream Fish Passage

- Downstream Fish Passage and Protection

- Watershed and Shoreline Protection

- Threatened and Endangered Species Protection

- Cultural and Historic Resource Protection**

- Recreational Resources**

**Not addressed defined by eight social

and environmental

impact criteria (listed

below), each addressed

through a series of goal

statements that define

the purpose or objective

that must be satisfied

(Sale et al. 2016).

A series of alternative

standards are provided

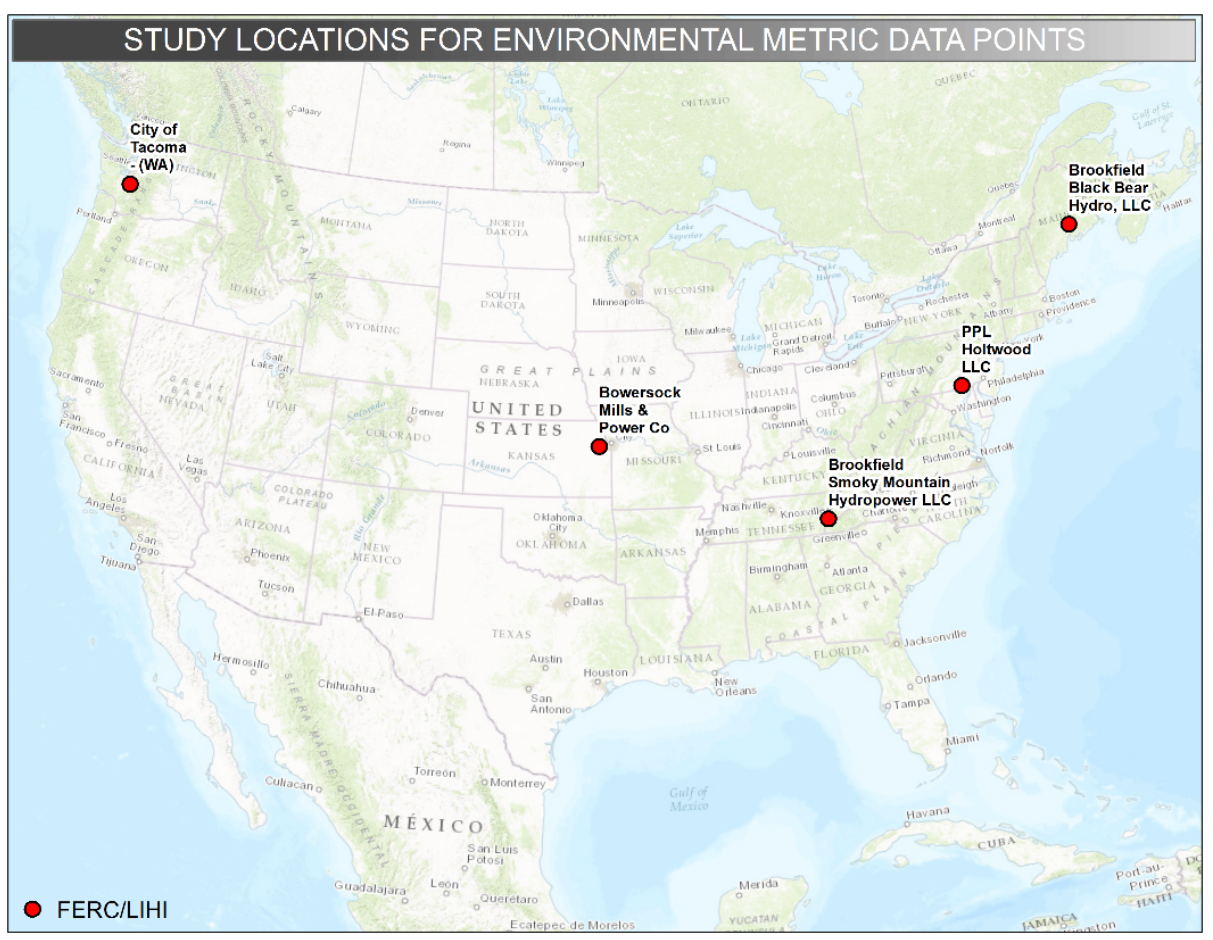


by which each criteria goal can be met. After consultation with LIHI staff, applicants prepare an application, which includes a description of project facilities and a standardized LIHI questionnaire. The questionnaire is structured to document how the applicant has addressed each of the eight criteria through a standard(s).

Additional supporting documents, such as fish passage plans, monitoring plans, and maps of facilities are provided.

For the same projects, FERC orders issuing new licenses and notices of environmental assessments (including the environmental impact assessment) were obtained from FERC e-library and reviewed. Typically, FERC orders are structured to provide a description of project facilities, followed by discussion of major environmental elements and stakeholder concerns, and then subsequent articles specifying the approved facilities and operations, including how environmental impacts are addressed. For a review of the detail of environmental information (including mitigation requirements) provided by FERC order approvals, please see Schramm et al. (2016). Because FERC specifies facility dimensions and capacities (e.g., dam storage) as apart of licensed projects, these elements are interpreted as metrics describing environmental impact along with traditional metrics (e.g., water temperature). For instance, if the licensee increases the capacity of a project, this will

Figure 7. Locations of non-Federal U.S. Hydropower projects certified by LIHI and likely require re-opening a license, as potential subsequent environmental impacts must be reassessed.

Table 2. List of five FERC/LIHI projects reviewed for environmental metrics*

\begin{tabular}{|c|c|c|c|c|c|c|c|c|}
\hline Hydropower Project & FERC No. & $\begin{array}{l}\text { LIHI } \\
\text { No. }\end{array}$ & $\begin{array}{l}\text { U.S. } \\
\text { State }\end{array}$ & River & Owner & $\begin{array}{l}\text { Capacity } \\
\text { (MW) }\end{array}$ & $\begin{array}{l}\text { Average } \\
\text { Annual } \\
\text { Gener-ation } \\
\text { (MWh) } \\
\end{array}$ & Metrics \\
\hline Bowersock Project & 13526 & 15 & $\mathrm{KS}$ & Kansas River & $\begin{array}{l}\text { Bowersock Mills } \\
\text { and Power } \\
\text { Company }\end{array}$ & 7 & 32,726 & $\begin{array}{l}71 \text { FERC, } 46 \\
\text { LIHI }\end{array}$ \\
\hline $\begin{array}{l}\text { Holtwood Hydroelectric } \\
\text { Project }\end{array}$ & 1881 & 116 & PA & $\begin{array}{l}\text { Susque-hanna } \\
\text { River }\end{array}$ & $\begin{array}{l}\text { PPL Holtwood, } \\
\text { LLC }\end{array}$ & 252 & 590,044 & $\begin{array}{l}132 \text { FERC, } 32 \\
\text { LIHI }\end{array}$ \\
\hline $\begin{array}{l}\text { Milford Hydroelectric } \\
\text { Project (includes Milford } \\
\text { Dam \& Gilmans Falls } \\
\text { Dam) }\end{array}$ & 2534 & 113 & ME & $\begin{array}{l}\text { Penobscot River; } \\
\text { Stillwater } \\
\text { Branch }\end{array}$ & $\begin{array}{l}\text { Black Bear Hydro } \\
\text { Partners, LLC }\end{array}$ & 7.8 & 55,186 & $\begin{array}{l}39 \text { FERC, } 16 \\
\text { LIHI }\end{array}$ \\
\hline $\begin{array}{l}\text { Nisqually Project (includes } \\
\text { La Grande and Alder } \\
\text { dams) }\end{array}$ & 1862 & 8 & WA & Nisqually River & City of Tacoma & 114 & 573,000 & $41 \mathrm{LIHI}$ \\
\hline Smoky Mountain Project & 2169 & 18 & $\mathrm{NC}, \mathrm{TN}$ & Little Tennessee & Brookfield Smoky & 376.6 & $1,361,821$ & 461 FERC, 30 \\
\hline
\end{tabular}

ORNL Catalog of Environmental Metrics for Hydropower - 13 


\section{*Source Documents:}

FERC. (1998). Order Issuing New License. Project No. 2534-005. Bangor-Hydro-Electric Company, Milford Project. April $20,1998$.

FERC. (2004). Notice of availability of environmental assessment. Alcoa Power Generating, Inc. Tapoco Project No. 2169-020. March 15, 2004

FERC. (2004). Notice of availability of final environmental assessment. Bangor-Pacific Hydro Associates. Project No. 2600-056. Final environmental assessment on ammendment of licenses: Veazie Hydroelectric Project, FERC No. 2403-048; Milford Hydroelectric Project, FERC No. 2534-068; West Enfield Hydroelectric Project, FERC No. 2600-056; Medway Hydroelectric Project, FERC No. 2666-023; Stillwater Hydroelectric Project, FERC No. 2712-055. April 18, 2005.

FERC. (2005). Order approving settlement and issuing new license. Project No. 2169-020. Alcoa Power Generating Inc., Tapoco Hydroelectric Project. January 25, 2005.

FERC. (2008). Draft Environmental Impact Statement for License Amendment. Holtwood Hydroelectric Project, FERC Project No. 1881-054, Pennsylvania. July 2008.

FERC. (2009). Order amending license and revising annual charges. Project No. 1881-054. PPL Holtwood, LLC. October 30, 2009.

FERC. (2010). Order Issuing Original License and terminating exemption from license. Project No. 13526-002; Project No. 2644-001. Bowersock Mills and Power Company. August 19, 2010.

FERC. (2010). Notice of availability of environmental assessment. Project No.13526-002. Bowersock Mills and Power Company. August 19, 2010 


\subsubsection{Peer-Reviewed Scholarly Literature}

Using guidelines established by the Center for Environmental Evidence (CEE 2013), a selection of peer-reviewed scholarly literature was identified to extract environmental metrics for Hydropower. The systematic reviews set forth rigorous and repeatable study inclusion criteria and documentation of environmental and hydropower search terms, search dates, and studies included so the review is replicable. This strict methodology of systematic review reduces biases in the studies selected for inclusion. This process has yielded 1,508 papers that are under detailed review extracting relevant environmental metrics. For more detailed explanation of this methodology and search terms please see Appendix A. From these a random subset of 247 papers were chosen to review. Of this subset, 97 articles listed in Appendix B and summarized in Appendix C contained environmental metrics. These environmental metrics were incorporated into a catalog including all information relevant to the metric (e.g., values of the metric, methods of data collection, temporal and spatial resolution).

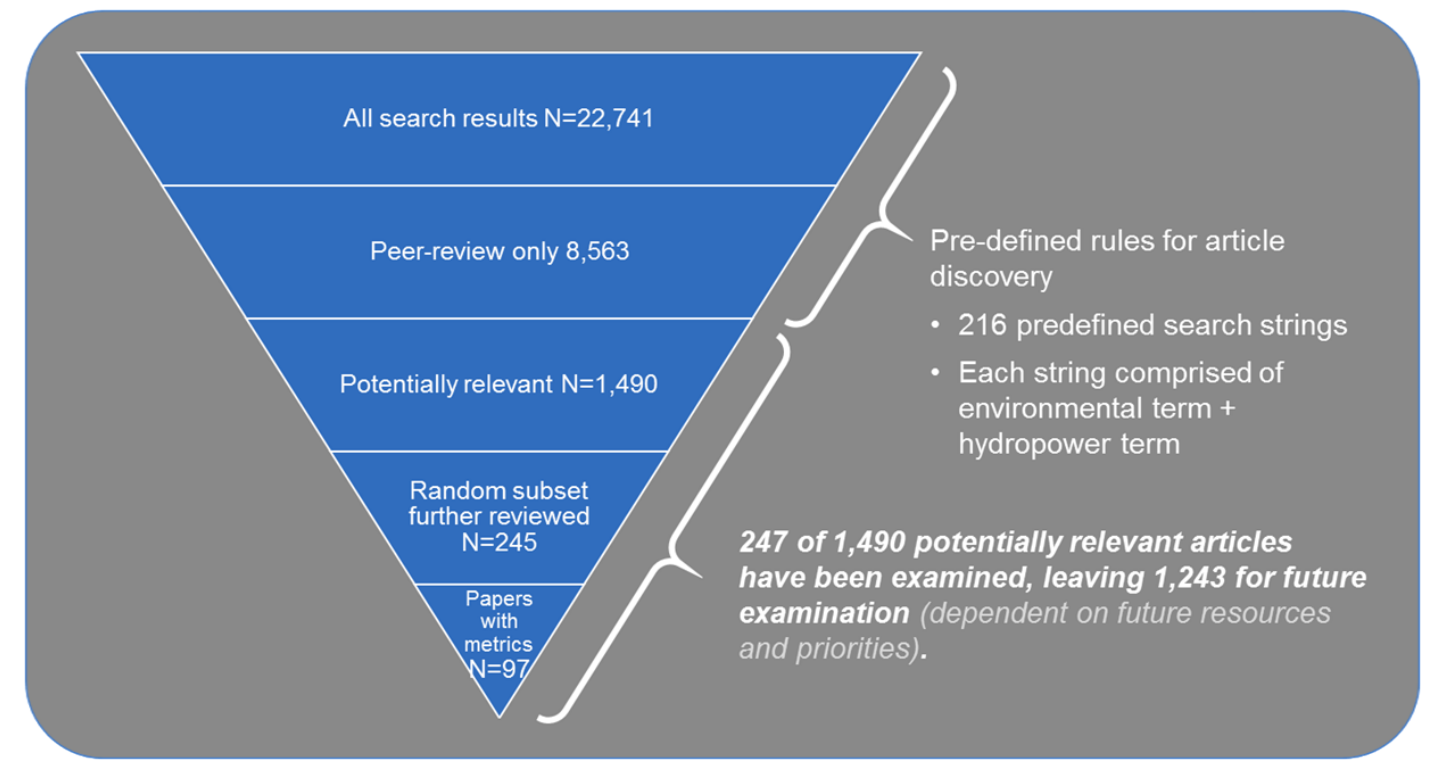

Figure 8 - Systematic Review of Scholarly Peer-Reviewed Literature

\subsubsection{Appropriate Use / Intended Use}

The definition of combinations of metrics that assess environmental sustainability in specific instances is the purview of stakeholders and is beyond the scope of this study. Follow-on research efforts may advance stakeholder-driven sustainability protocols. This study will not address the role of hydropower development in altering carbon cycling, but the metrics developed in this study may prove useful within that research domain. 


\section{OTHER TYPES OF INFORMATION EXTRACTED}

\section{$2.1 \quad$ PROJECT LOCATION}

As environmental metric information was gathered, the site locations of specific studies (Figure 9) were linked in the database to the National Hydropower Asset Assessment Program (NHAAP) database and National Inventory of Dams (NID) for hydropower facilities in the United States and the Global Reservoir and Dam (GRanD) database for non-U.S. hydropower projects. Online searches were conducted for hydropower projects that were not listed in any of these datasets. We also used NHAAP, NID and GRanD obtain ancillary information such as generating capacity, generation, dam characteristics, and reservoir properties:

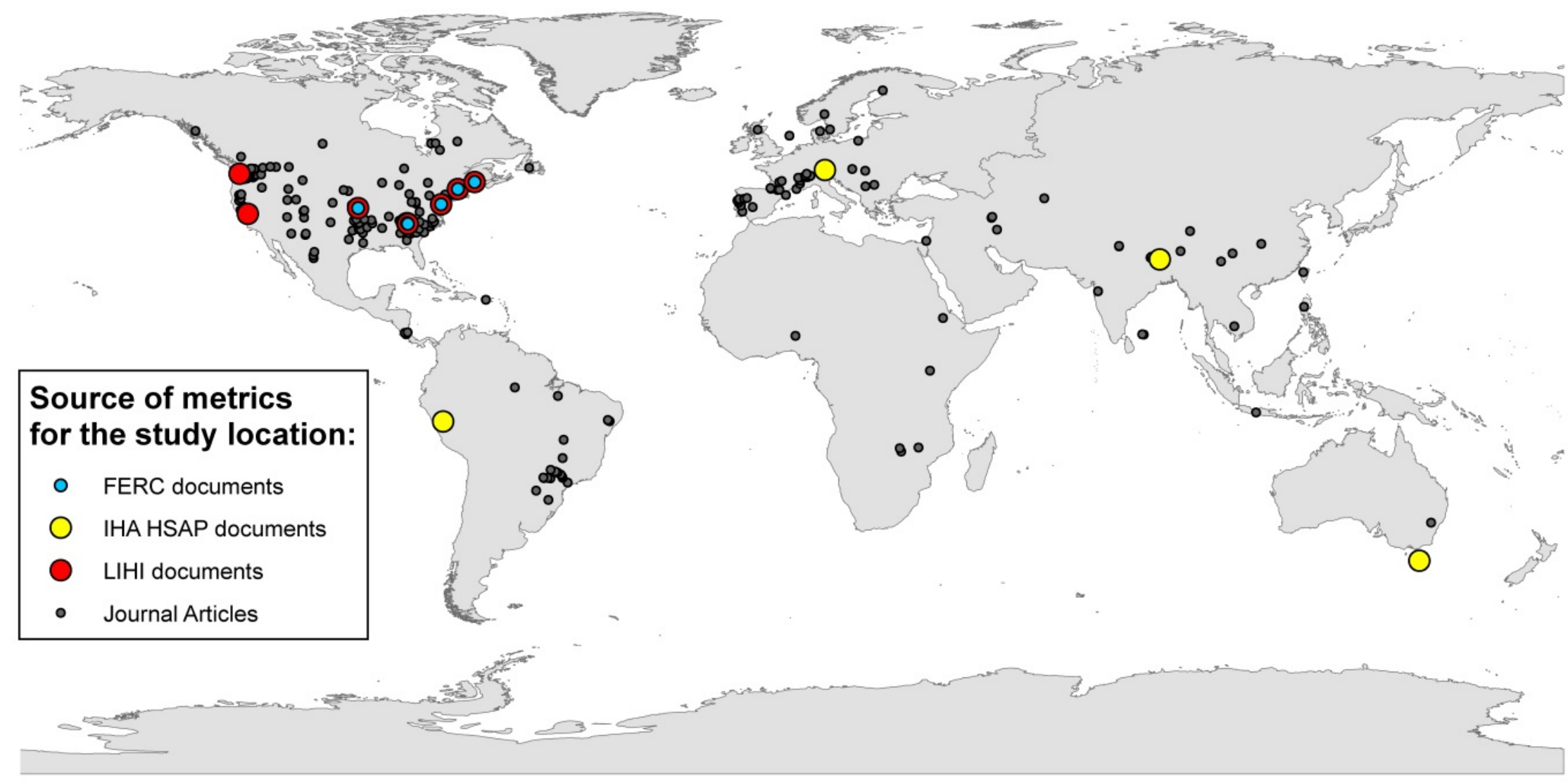

Figure 9 - Map showing the 231 study locations used to collect the metrics recorded for this analysis of the environmental effects of hydropower. 


\subsection{PROJECT STATUS}

Six hydropower life cycles have been defined by the ORNL Standard Modular Hydropower project (reference): (1) initial project determination, (2) permitting \& regulatory approval, (3) pre-commissioning activities, (4) construction, (5) operations \& maintenance, and (6) decommissioning. During metric extraction, the life cycle stage of the dam at the time that the referenced document was prepared is captured.

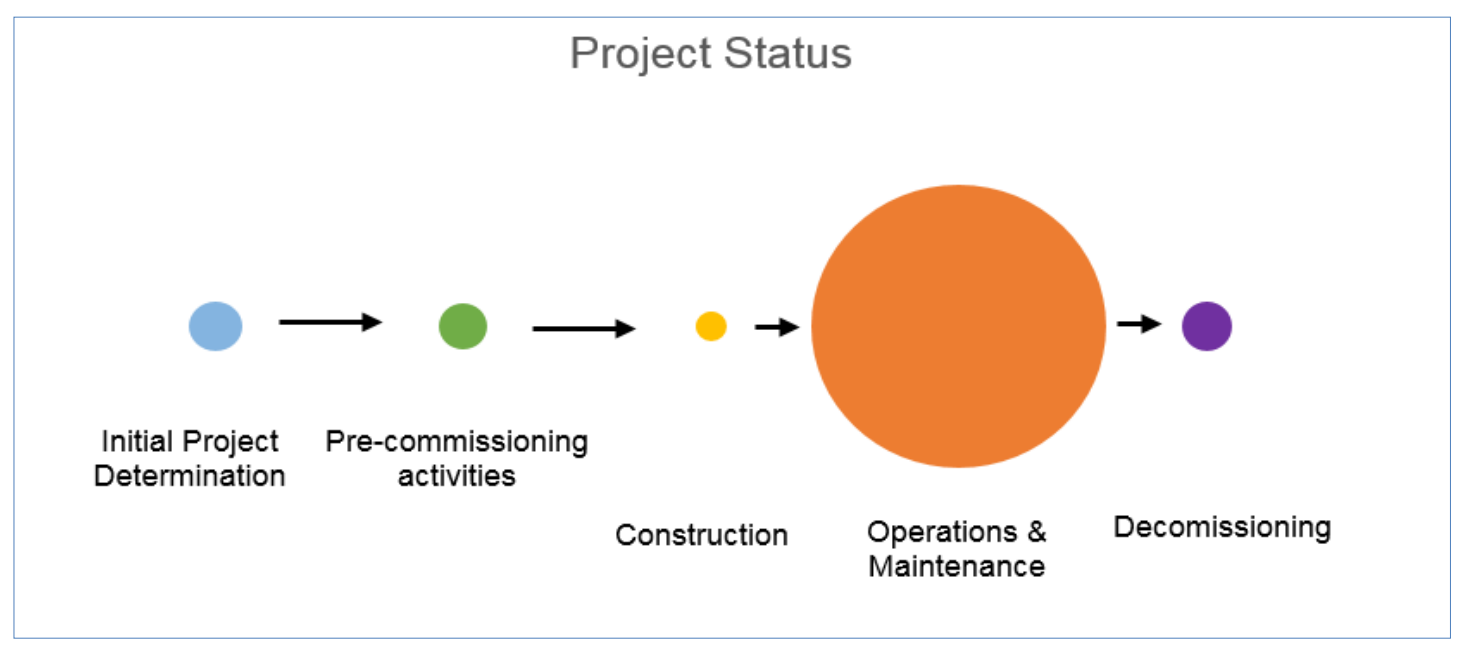

Figure 10 - The represented phases of hydropower development associated with the extracted metrics. 


\subsection{SPATIAL SCALE}

Metrics are applied at different scales, so we recorded spatial information according to the names and definitions shown in Table 2 . The frequency of the environmental metrics captured across these different scales is shown in Figure 12.

Table 3 Spatial scales of environmental metrics related to hydropower

\begin{tabular}{|c|c|c|}
\hline Spatial Scale & Definition & Examples \\
\hline Within dam & Metrics associated with internal dam components & Turbine type \\
\hline Dam & Metrics associated with the dam itself & Fish passage; Seismic stability \\
\hline Reservoir & Metrics associated with the impoundment located immediately upstream of the dam & $\begin{array}{l}\text { Shoreline erosion; Algal blooms; Siltation rates; } \\
\text { Offgassing }\end{array}$ \\
\hline River_downstream & Metrics associated with the river downstream of the dam, including the tailwater & $\begin{array}{l}\text { Flow rate; Dissolved oxygen levels; Water } \\
\text { temperature; Fish counts }\end{array}$ \\
\hline River_upstream & Metrics associated with the upstream mainstem and tributaries & $\begin{array}{l}\text { Flow rate; Dissolved oxygen levels; Water } \\
\text { temperature; Fish counts }\end{array}$ \\
\hline Basin & Metrics associated with the watershed in which the hydropower project is located & $\begin{array}{l}\text { Water consumption rates; Number of stream } \\
\text { tributaries }\end{array}$ \\
\hline Landscape & $\begin{array}{l}\text { Metrics associated with the terrestrial landscape surrounding the hydropower } \\
\text { project }\end{array}$ & $\begin{array}{l}\text { Percent forest cover; Number of road crossings; } \\
\text { Miles of transmission lines }\end{array}$ \\
\hline Project & Metrics associated with the entire hydropower project (e.g., multiple dams) & Water temperature; Fish condition; Genetic diversity \\
\hline
\end{tabular}

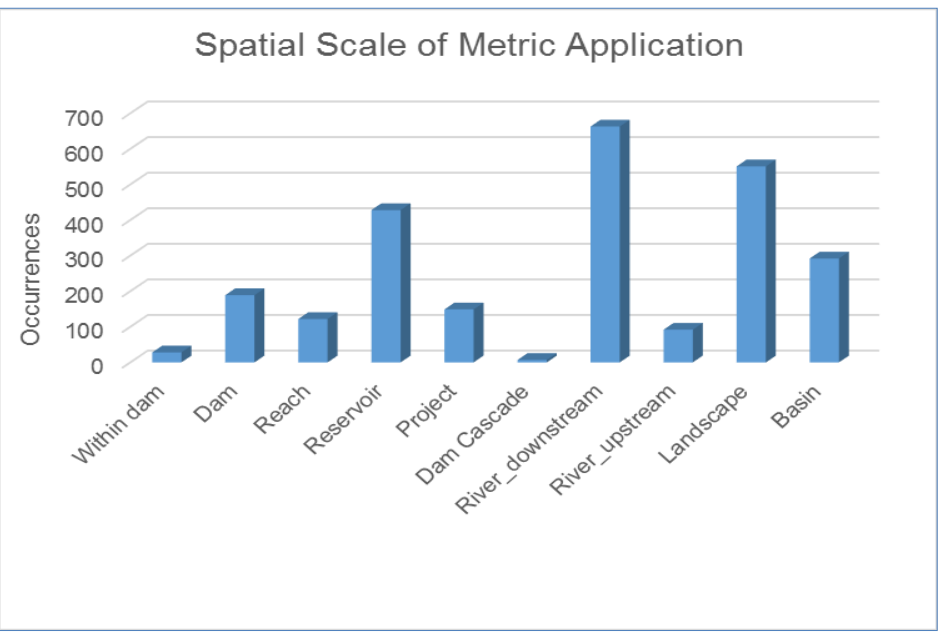

Figure 11 - Spatial scale associated with catalogued metrics.

ORNL Catalog of Environmental Metrics for Hydropower - 18 


\section{RESULTING SET OF ENVIRONMENTAL METRICS}

To capture a broad swath of measurements from multiple sectors concerned with potential hydropower impacts, we based our literature review of environmental metrics on a combination of FERC regulatory documents, LIHI and IHA HSAP certification documents, and peer-reviewed scientific journal articles. Our review of the 117 documents yielded a set of 3183 environmental metrics recorded during a variety of studies related to dams and hydropower projects. These metrics were related to 231 dams and study locations worldwide (Figure 9). Note that several of these points indicate multiple small dams aggregated within a single study. Most of the study sites were in North America (121) and Europe (53), followed by South America (29), Asia (20), Africa (6) and Australia (2). The dams ranged in size from small earthen dams (and even one inflatable dam) built solely for irrigation, flood control, and/or recreational purposes to powered dams with capacities ranging from micro size (i.e., less than $0.1 \mathrm{MW}$ ) to as much as 22,500 MW. The literature review included dams assessed across all lifecycle stages (Figure 10), but most of the metrics were collected from dams in the operations and maintenance stage.

The geographic distribution, size and ownership of the U.S. dams captured by this literature review relative to the entire U.S. hydropower fleet is shown in Figure 12. Note that the nonpowered dams captured by the literature review are dams currently managed for flood control, irrigation and/or recreational purposes with no electric power generation as well as a few dams that no longer generate power because they have been decommissioned). This comparison shows that our literature review ended up being biased toward larger, federally owned dams. Small U.S. dams (0.1 to $10 \mathrm{MW})$ seem to be particularly underrepresented by this dataset of environmental metrics. We were unable to do a similar comparison for the non-U.S. dams due to insufficient hydropower fleet data at the global scale.

A summary of the 3183 captured environmental metrics by category, sector and type is shown in a "dashboard" format in Figure 13. Frequency distributions of the metrics by category and source document type are provided in Figure 14. Irrespective of literature source, most metrics were related to Water Quantity and Water Quality categories, many metrics were related to Biota \& Biodiversity, and relatively few metrics were related to the other four categories of Geomorphology, Land Cover, Connectivity \& Fragmentation, and Infrastructure \& Design. An analysis of the metric distributions broken out for each document type shows that there was not much consistency between the source documents (Figure 14). The journal articles and IHA HSAP documents focused more on Water Quality than Water Quantity. A majority of the Geomorphology metrics came from journal articles and FERC documents, while none were identified in the LIHI documents. Infrastructure \& Design metrics were much more prevalent in the FERC and LIHI documents than the journal articles and IHA HSAP documents.

The geographic distribution of the collected metrics by continent and U.S. region is provided in Figure 15. Figure 15A shows a predominance of water quantity and water quality metrics across all continents with a more even mix of the two categories across Europe, South America, Africa and Asia. Given that the pie sizes indicate the relative number of metrics collected across each continent, one can see that the environmental metrics captured by the database are largely from North America and Europe with very few from Oceania. A distribution of the metrics by category across seven U.S. regions reflecting aggregations of river basins mapped by the U.S. Geological Survey (Figure 15B) shows that a substantial number of environmental metrics were captured from documents pertaining to the Southeastern U.S. and that water quantity metrics predominated in all regions except for the Northeast. The Northeastern U.S. showed a more even distribution of metrics across the seven categories, with the largest number of metrics gathered in the category of Biota and Biodiversity.

Examination of the entire set of collected environmental metrics showed coalescence around 45 parameters, or subcategories, of environmental metrics (Table 4) and that most of these subcategories were represented by all three metric types. Details about metrics collected in each of the seven categories are provided in Sections 3.1-3.7. 

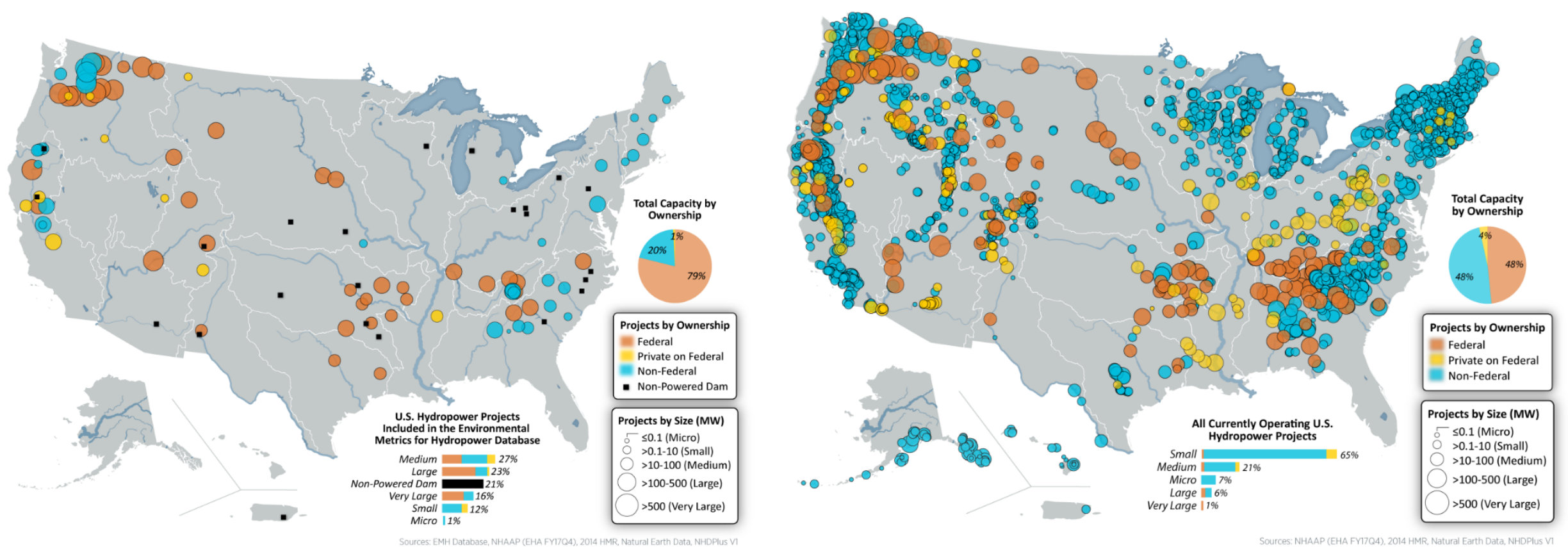

Figure 12 - Size and ownership distribution of U.S. hydropower projects captured by this environmental metrics for hydropower literature review (left) relative to the entire U.S. hydropower fleet (right). 


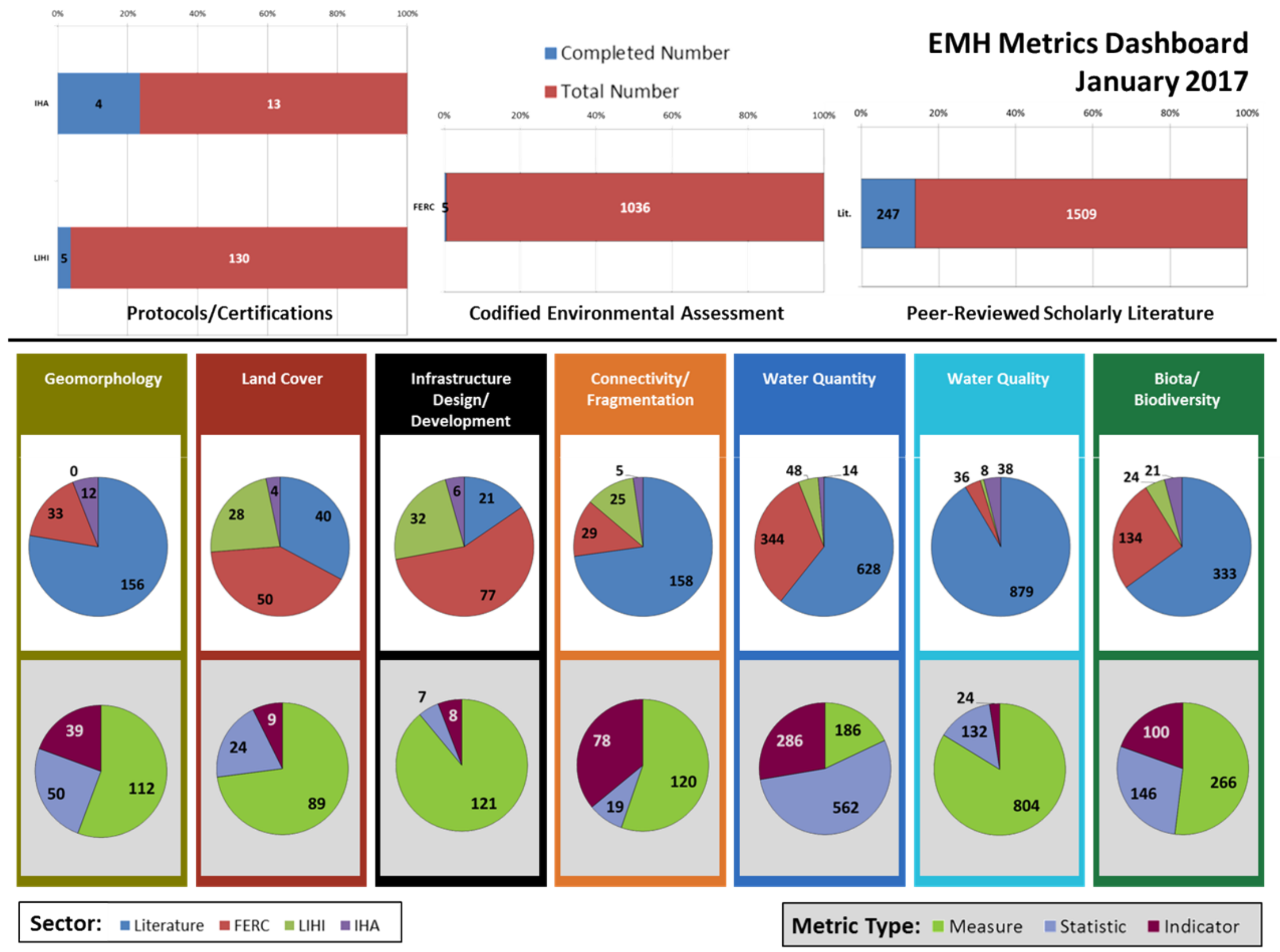

Figure 13 - Summary of captured environmental metrics by category, sector and type.

ORNL Catalog of Environmental Metrics for Hydropower - 21 
All Documents (3183 metrics)

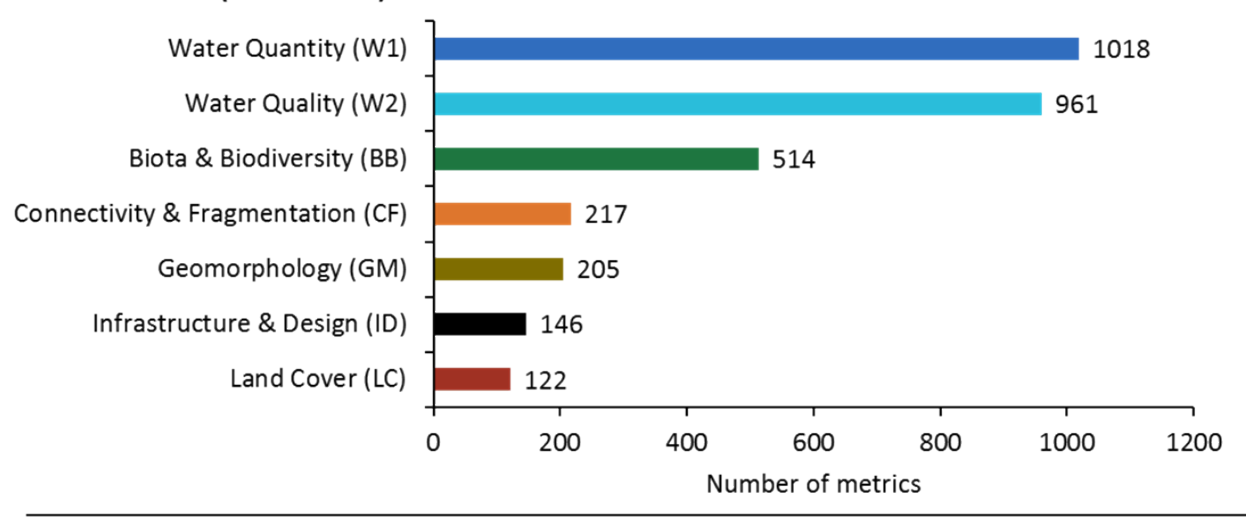

Journal Articles (2215 metrics)

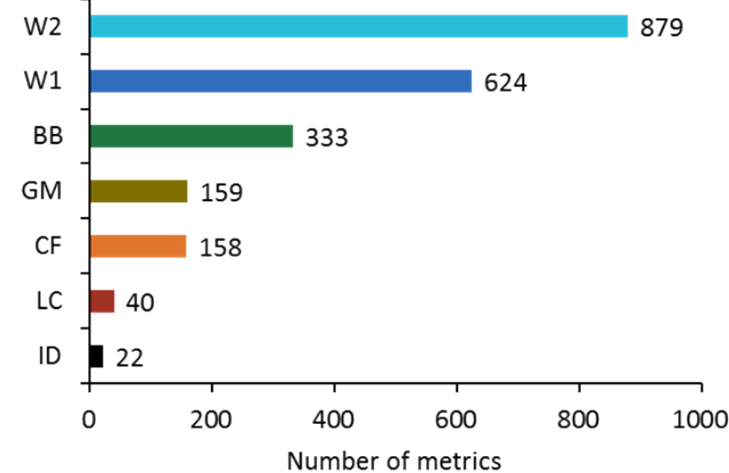

IHA HSAP Documents (100 metrics)

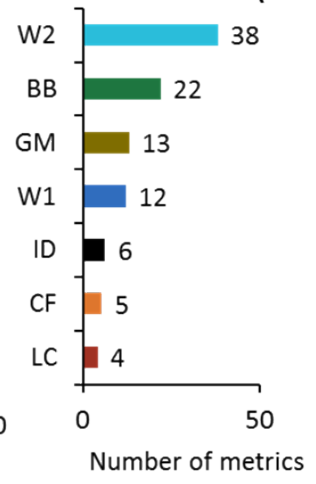

FERC Documents (783 metrics)

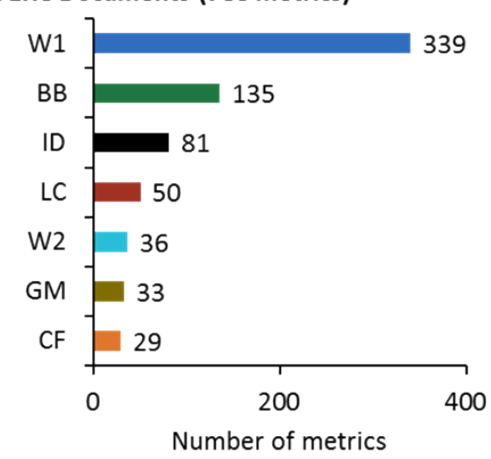

LIHI Documents (165 metrics)

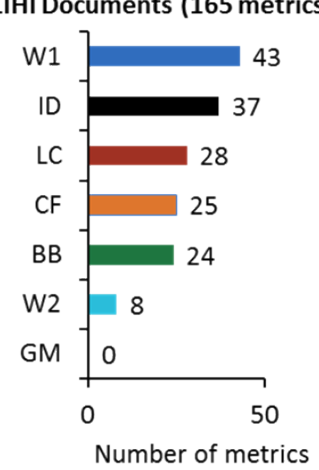

Figure 14- Distribution of the environmental metrics by category and document type

ORNL Catalog of Environmental Metrics for Hydropower - 22 

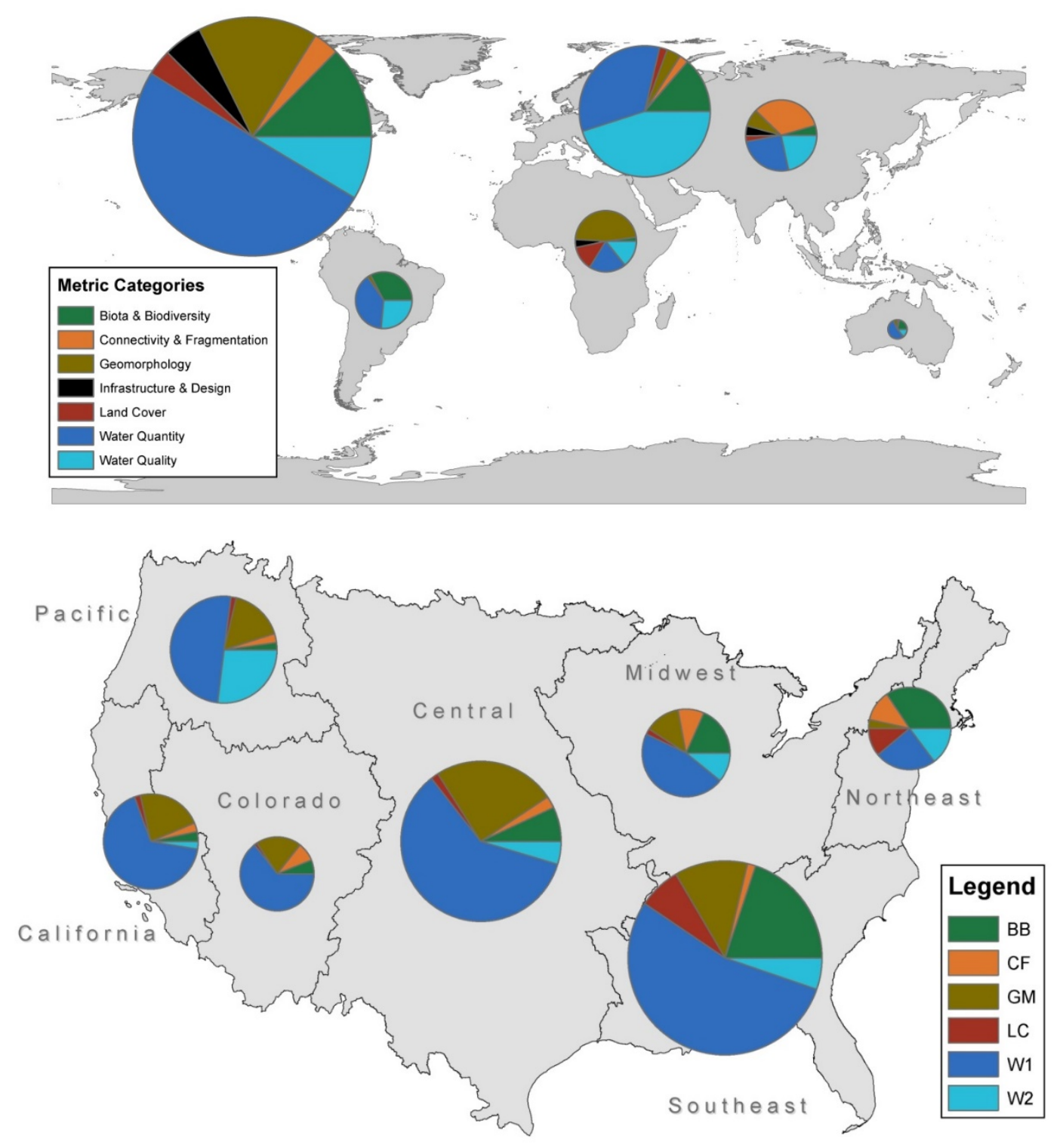

Figure 15- Geographic distribution of the collected environmental metrics by continent (top) and by U.S. region (bottom).

ORNL Catalog of Environmental Metrics for Hydropower - 23 
Table 4 Emergent sub-categories (45) of the environmental metrics (3183) collected through this literature review of 117 documents. Metrics totals are also shown by type: $\mathbf{M}=$ Measures, $\mathrm{S}=$ Statistics, and $\mathrm{I}=$ Indicators.

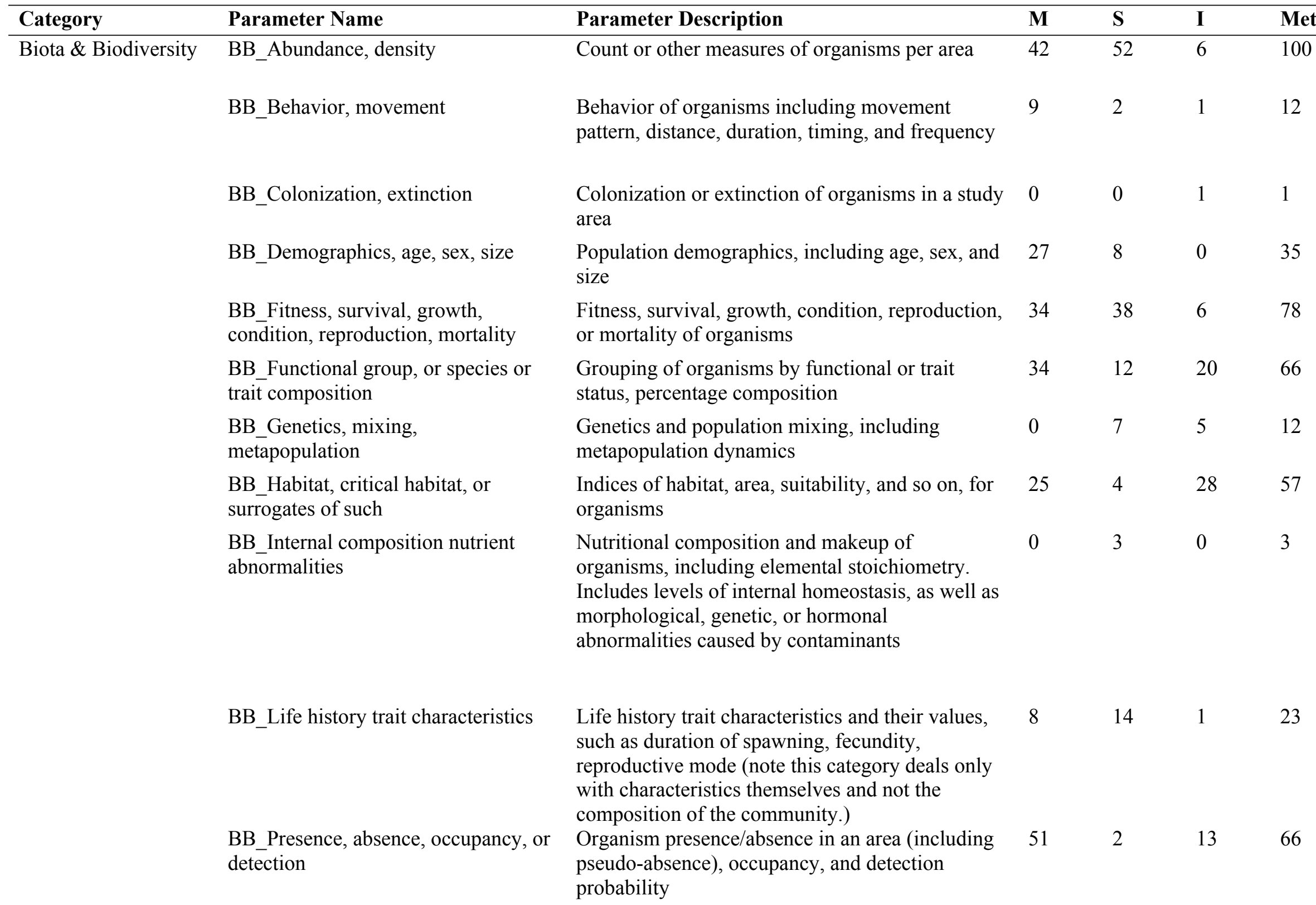

ORNL Catalog of Environmental Metrics for Hydropower - 24 
BB_Richness, diversity, evenness, or IBI types of measures

\section{Connectivity \& Fragmentation}

Geomorphology

GM_Catchment and basin attributes

GM_Channel

GM_Flooplain valley

GM_Sediment and substrate

Infrastructure \& Design

ID_Dam attributes

ID_Fish passage

ID_Turbine
Species richness, diversity, evenness, or indices-

or more components of the biotic community

Some aspect of area of river basin

8

Fragment length, dendritic connectivity index, projects

Mitigated fish passage, including presence of upstream or downstream passage or length of bypass

Upland soil characteristics, topography, and landscape erodibility metrics that could influence soil erosion and wasting related and subsequent sedimentation related to hydropower development

Channel properties such as bankfull width, wetted width, bankfull discharge, channel slope, braided channel, channelization

Metrics related to channel confinement, entrenchment, migration, etc.

Sediment and substrate properties such as substrate particle size, bedload, sediment entrainment or deposition, bedrock composition Head, dam height, spill gate type, bar rack, and so on

Characteristics of fish passage structures such as slope, velocity, and discharge

Turbine characteristics including forces in the turbine environment such as pressure, shear,

cavitation, turbine type, turbine speed, blade strike 
LC_Floodplain or riparian vegetation

LC_Land cover class

LC_Protected land

\section{LC_Reservoir inundation}

Water Quantity

W1_Basin attributes

W1_Diversion

W1_Downstream discharge and hydrology

\section{W1_Groundwater \\ W1_Reservoir hydrology}

W1_Upstream regulation and inflow

Water Quality project as whole, not related to reservoir

inundation or land cover

Properties of floodplain or riparian vegetation such as riparian encroachment or floodplain area

Type of land cover, changes in land cover Spatial properties of protected lands including losses or increases

Reservoir area, upland or floodplain inundation, biomass inundated/lost

Attributes related to factors that influence hydrology (or were used in the context of hydrology), such as climate and precipitation

Quantitative properties of diversions such as volume or discharge of diversion or water for other uses

Measures that describe the magnitude, frequency, duration, periodicity, and timing of flows downstream of a hydropower facility, including changes to these characteristics

Groundwater characteristics

Reservoir hydrological characteristics such as residence time, reservoir fluctuation, reservoir surface area, or degree of regulation

Measures describing the magnitude, frequency, duration, periodicity, and timing of flows upstream of a hydropower facility, including changes to these characteristics

Algal concentration including measures of primary productivity such as chlorophyll A or cyanotoxin.

Characteristics including $\mathrm{pH}$, alkalinity

ORNL Catalog of Environmental Metrics for Hydropower - 26 
0

W2_Dissolved oxygen

W2_Ecosystem function

W2_Gas emissions

W2_Nutrients

W2_Organic material

W2_Other elements

W2_Pollutants

W2_Solid transport, turbidity, and conductivity
Dissolved oxygen in water

Ecosystem vital rates and processes, including gross primary productivity, respiration,

biochemical oxygen demand

Concentration and ebullution of water-origin greenhouse gases

All non-rare elements essential to life: nitrogen, phosphorous, inorganic carbon, potassium, sulfur, and magnesium compounds (rare essential elements are included in "other elements")

Dissolved organic carbon and other organic nonpollutants

Elements and compounds that are not listed on the EPA Toxic and Priority Pollutants list

Pollutants listed on the EPA Toxic and Priority Pollutants list that are not included in other EMH categories

Descriptions of dissolved and suspended solids in water such as turbidity, suspended or dissolved solids, conductance

$\begin{array}{llll}9 & 9 & 2 & 20\end{array}$

$\begin{array}{llll}5 & 7 & 1 & 13\end{array}$

99

Water temperature 


\subsection{GM_Geomorophology}
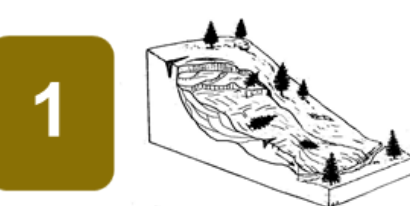

Geomorphology is the dynamic evolution of topographic and bathymetric features created within an ecosystem. Hydropower development can disrupt a river system's geomorphologic equilibrium through altered sediment and flow regimes. These changes have the potential to impact the availability and quality of habitats for plants and animals within the system.

\section{GM Geomorphology}

\begin{tabular}{|c|c|c|}
\hline Sector & \# of studies & $\#$ of metrics \\
\hline IHA & 4 & 12 \\
\hline LIHI & 5 & 0 \\
\hline FERC & 5 & 33 \\
\hline $\begin{array}{l}\text { Peer-Reviewed } \\
\text { Literature }\end{array}$ & 247 & 156 \\
\hline Totals & 261 & 201 \\
\hline
\end{tabular}

Geomorphology parameter frequency at study-level (extraction table)

Primary

Category Parameter

Geomorphology GM_Catchment and basin attributes

GM_Channel

GM_Flooplain valley

GM_Sediment and substrate
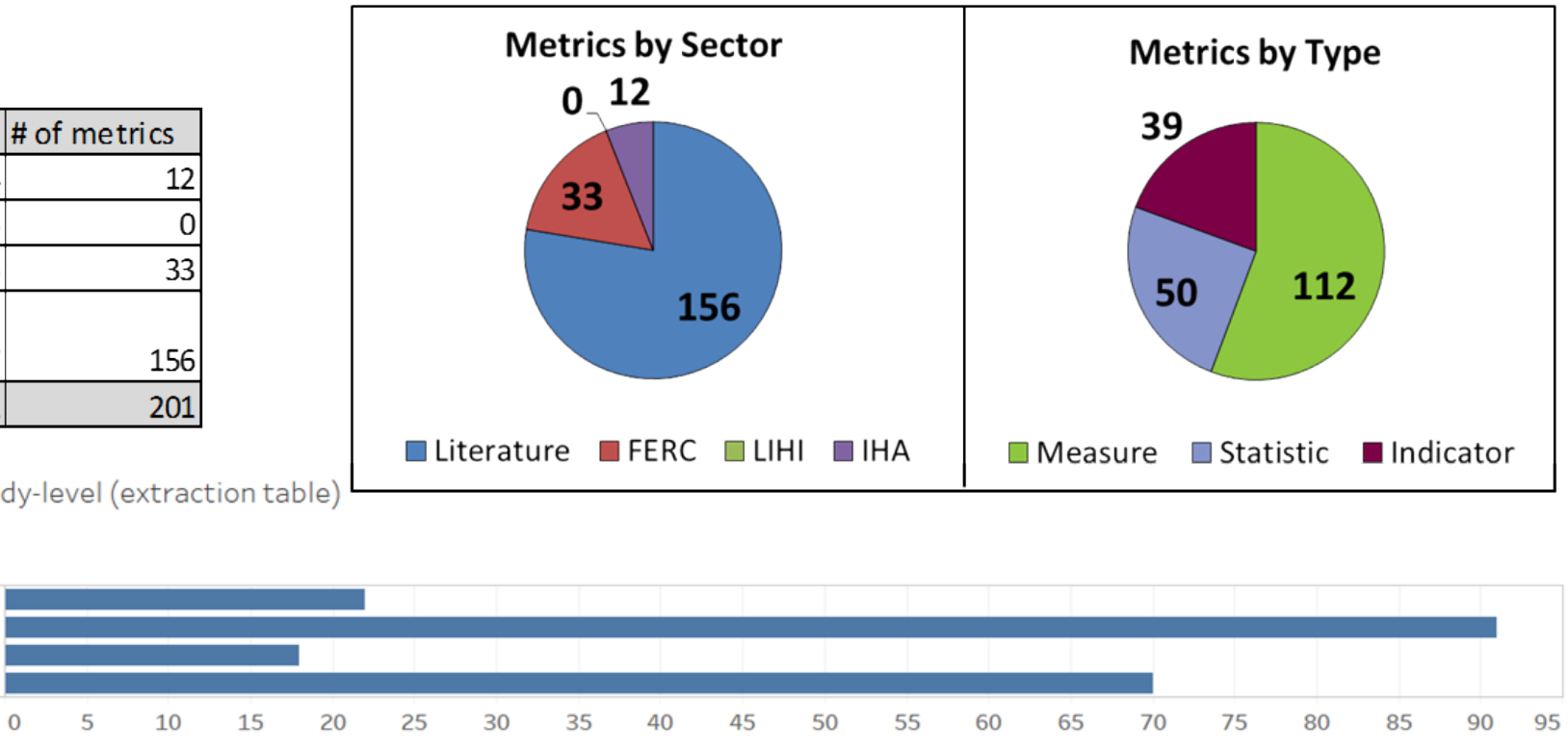

Spatial scales for geomorphology represented at study-level (extraction table)

Primary

Category Spatscale

Geomorphology Basin

Landscape

Project

Reach

Reservoir

River_downstream

River_upstream Within dam

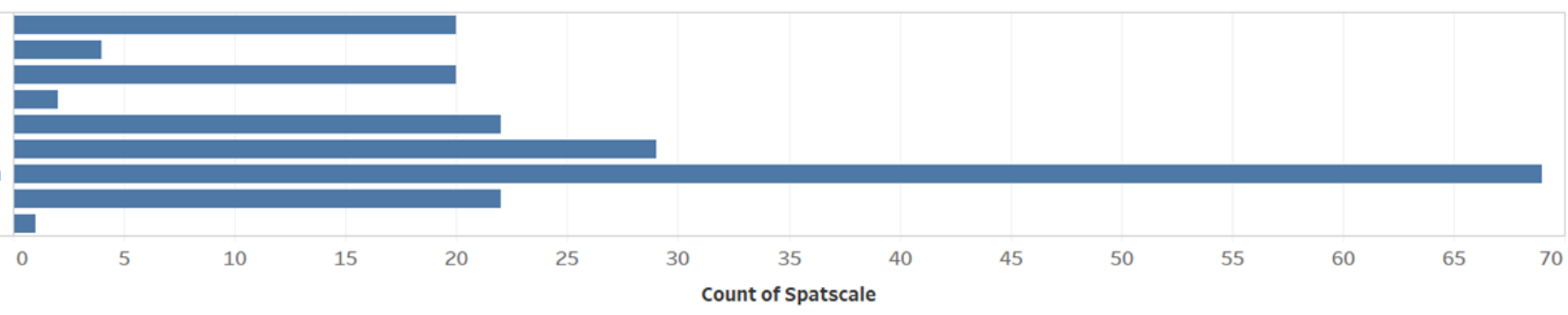

ORNL Catalog of Environmental Metrics for Hydropower - 28 
3.1.1 GM_Catchment and basin attributes

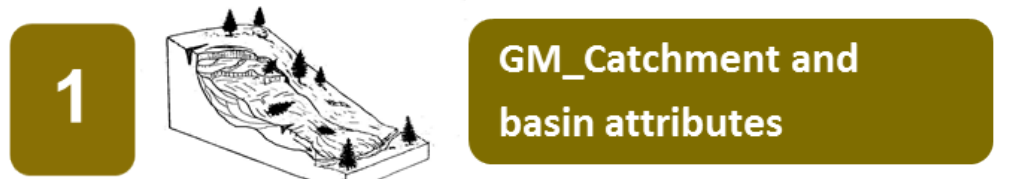

GM Geomorphology

\begin{tabular}{|l|r|r|}
\hline Sector & studies metrics \\
\hline IHA & 4 & 2 \\
\hline LIHI & 5 & 0 \\
\hline FERC & 5 & 4 \\
\hline \begin{tabular}{l|r} 
Peer-Reviewed \\
Literature
\end{tabular} & 247 & 16 \\
\hline \multicolumn{1}{|r|}{ Totals } & 261 & 22 \\
\hline
\end{tabular}

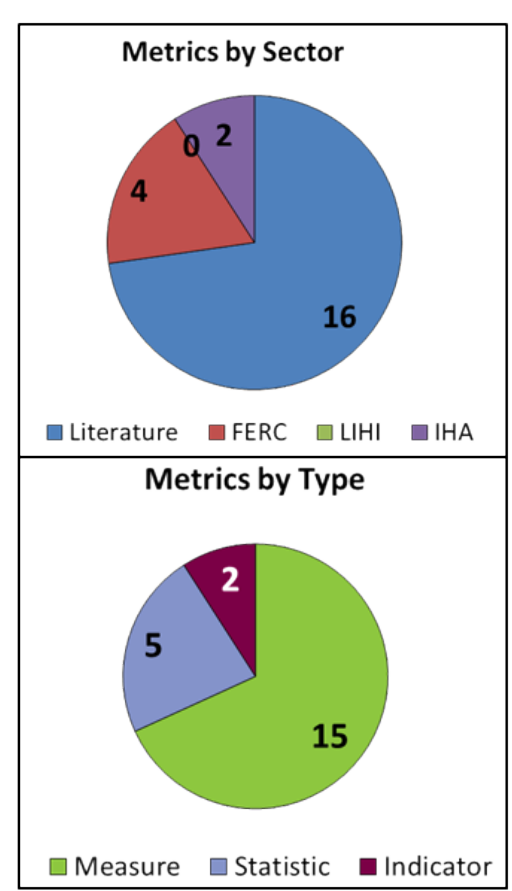

Upland soil characteristics, topography, and landscape erodibility metrics that could influence soil erosion and wasting related and subsequent sedimentation related to hydropower development

\begin{tabular}{|l|l|}
\hline Metric Type & \multicolumn{1}{|c|}{ Metric Name } \\
\hline Measure & Basin area \\
\hline Measure & Catchment size \\
\hline Measure & Channel slope \\
\hline Measure & Digital elevation model \\
\hline Measure & Geology type \\
\hline Measure & Local al titude \\
\hline Measure & Slope \\
\hline Measure & Soil type \\
\hline Measure & Soils \\
\hline Measure & Surface geology \\
\hline Statistic & Catchment altitude-- average \\
\hline Statistic & Matric suction \\
\hline Statistic & Permeability function \\
\hline Statistic & Soil-water characteristic curve \\
\hline Statistic & Water content-- volumetric \\
\hline Indicator & Baseline erosion and sedimentation \\
\hline Indicator & Erosion study \\
\hline
\end{tabular}

ORNL Catalog of Environmental Metrics for Hydropower - 29 


\subsubsection{GM_Channel}

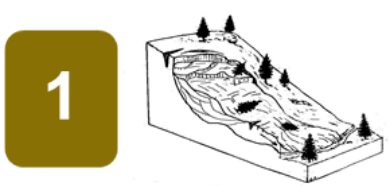

GM Geomorphology
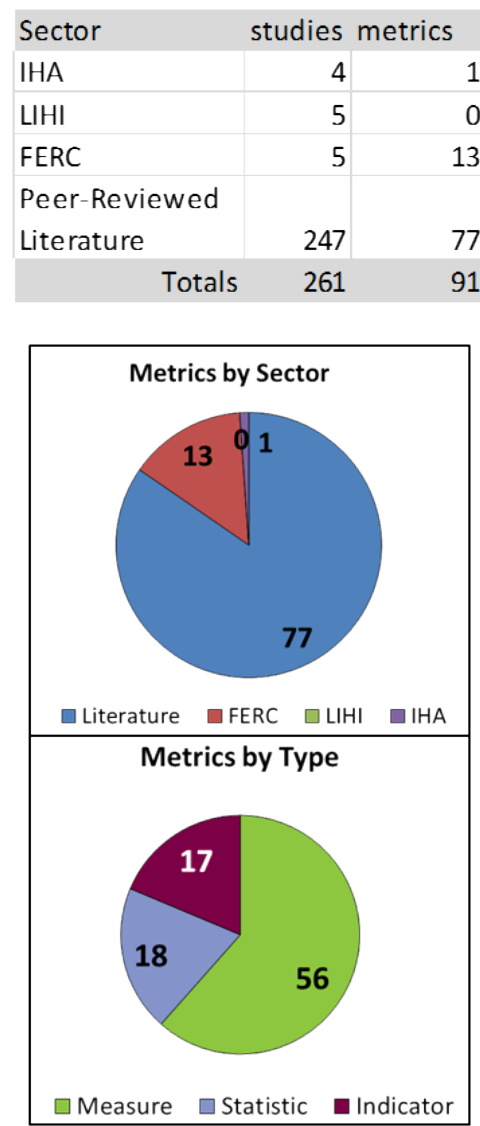

GM_Channel

Channel properties such as bankfull width, wetted width, bankfull

discharge, channel slope, braided channel, channelization

\begin{tabular}{|l|l|}
\hline Metric Type & \multicolumn{1}{|c|}{ Metric Name } \\
\hline Measure & Bank slope \\
\hline Measure & Bankfull cross sectional area \\
\hline Measure & Bankfull depth \\
\hline Measure & Bankfull width \\
\hline Measure & $\begin{array}{l}\text { Channel cross-section-- distance from } \\
\text { left bank }\end{array}$ \\
\hline Measure & Channel elevation \\
\hline Measure & $\begin{array}{l}\text { Channel elevation cross-section } \\
\text { longitudinal }\end{array}$ \\
\hline Measure & Channel gradient \\
\hline Measure & Channel pattern \\
\hline Measure & Channel soil type \\
\hline Measure & Channel width \\
\hline Measure & Channel width active \\
\hline Measure & Elevation of sandbar above sea level \\
\hline Measure & Engineered surface area \\
\hline Measure & Erosion coastal \\
\hline Measure & Flow vel ocity \\
\hline Measure & High bar area \\
\hline Measure & High-flow channel area \\
\hline Measure & Island area \\
\hline Measure & Low bar area \\
\hline Measure & Low-flow channel area \\
\hline Measure & Reach length \\
\hline Measure & River slope \\
\hline Measure & River type \\
\hline Measure & River width \\
\hline Measure & Sediment deposition \\
\hline Measure & Water depth \\
\hline Measure & Water depth-- regulated stream \\
\hline Measure & Water depth--unregulated stream \\
\hline Measure & Water velocity \\
\hline Measure & Water velocity-- channel \\
\hline Measure & $\begin{array}{l}\text { Water veocity surface-- regulated } \\
\text { stream }\end{array}$ \\
\hline
\end{tabular}

\begin{tabular}{|l|l|}
\hline Metric Type & \multicolumn{1}{|c|}{ Metric Name } \\
\hline Measure & $\begin{array}{l}\text { Water veocity surface-- unregulated } \\
\text { stream }\end{array}$ \\
\hline Measure & Wetted perimeter \\
\hline Measure & Wetted width and depth \\
\hline Measure & Width channel cross-section \\
\hline Statistic & Channel width active \\
\hline Statistic & Channel width active-- maximum \\
\hline Statistic & Channel width active-- minimum \\
\hline Statistic & GlS cells outside of thres hold-- percent \\
\hline Statistic & Pool, run, riffle-- percent \\
\hline Statistic & Stream power \\
\hline Statistic & Stream width-- average \\
\hline Statistic & Water depth-- average \\
\hline Statistic & Water velocity \\
\hline Statistic & Water velocity-- average \\
\hline Indicator & Bank erodability \\
\hline Indicator & Bank erosion \\
\hline Indicator & Bathymetric surveys \\
\hline Indicator & Bed degradation \\
\hline Indicator & Channel cross-section-- measurements \\
\hline Indicator & Channel elevation change \\
\hline Indicator & Channel elevation-- change \\
\hline Indicator & Channel erosion \\
\hline Indicator & Channel width active-- percent change \\
\hline Indicator & Channelization presence \\
\hline Indicator & Lateral migration \\
\hline Indicator & Net land loss \\
\hline Indicator & Rate land loss \\
\hline Indicator & U-shaped cross section \\
\hline Indicator & Width channel cross-section-- change \\
\hline & \\
\hline
\end{tabular}

Frequencies based on EMH Catalog Contents as of January 31, 201

ORNL Catalog of Environmental Metrics for Hydropower - 30 


\subsubsection{GM_Floodplain valley}

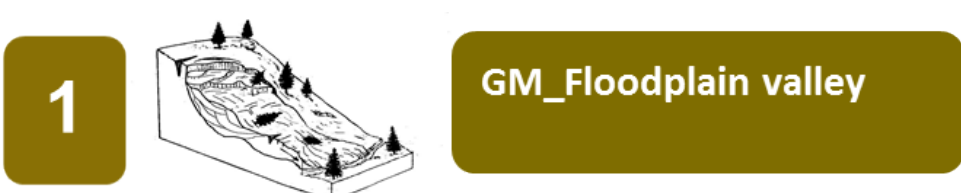

Metrics related to channel confinement, entrenchment, migration, etc.

\section{GM Geomorphology}

\begin{tabular}{|l|r|r|}
\hline Sector & studies metrics \\
\hline IHA & 4 & 0 \\
\hline LIHI & 5 & 0 \\
\hline FERC & 5 & 2 \\
\hline \begin{tabular}{l|r|} 
Peer-Reviewed \\
Literature
\end{tabular} \\
\hline \multicolumn{1}{|r|}{ Totals } & 247 & 16 \\
\hline
\end{tabular}

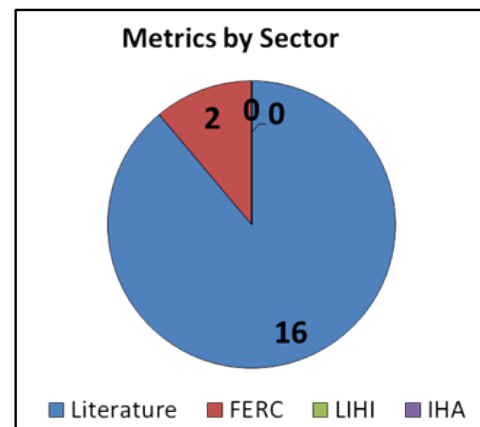

Metrics by Type

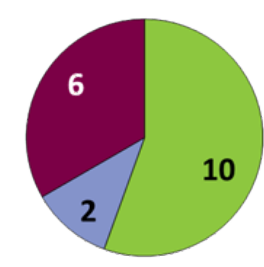

$\square$ Measure $\square$ Statistic $\square$ Indicator

\begin{tabular}{|c|c|}
\hline Metric Type & Metric Name \\
\hline Measure & Floodplain active area \\
\hline Measure & Floodplain characteristics \\
\hline Measure & Floodplain extent \\
\hline Measure & Floodplain inactive area \\
\hline Measure & Shoreline length \\
\hline Measure & Valley form \\
\hline Measure & Valley slope \\
\hline Statistic & $\begin{array}{l}\text { Accumulated deformation } \\
\text { landface }\end{array}$ \\
\hline Statistic & Watershed erosion \\
\hline Indicator & Impact factor \\
\hline Indicator & $\begin{array}{l}\text { Precipitation patterns for } \\
\text { estimating sediment yield-- } \\
\text { qualitative }\end{array}$ \\
\hline Indicator & Ratio of factor of safety-- change \\
\hline Indicator & $\begin{array}{l}\text { Runoff patterns for estimating } \\
\text { sediment yield-- qualitative }\end{array}$ \\
\hline Indicator & Soil stratigraphy \\
\hline Indicator & Upland erosion \\
\hline
\end{tabular}

ORNL Catalog of Environmental Metrics for Hydropower - 31 
3.1.4 GM_Sediment and substrate

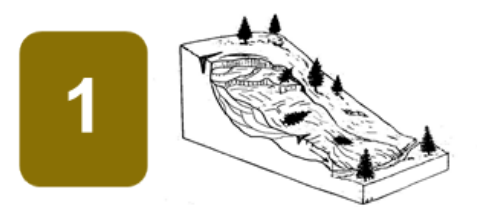

GM Geomorphology

\begin{tabular}{|l|r|r|}
\hline Sector & studies metrics \\
\hline IHA & 4 & 9 \\
\hline LIHI & 5 & 0 \\
\hline FERC & 5 & 14 \\
\hline \begin{tabular}{l|r} 
Peer-Reviewed \\
Literature
\end{tabular} & 247 & 47 \\
\hline \multicolumn{1}{|r|}{ Totals } & 261 & 70 \\
\hline
\end{tabular}

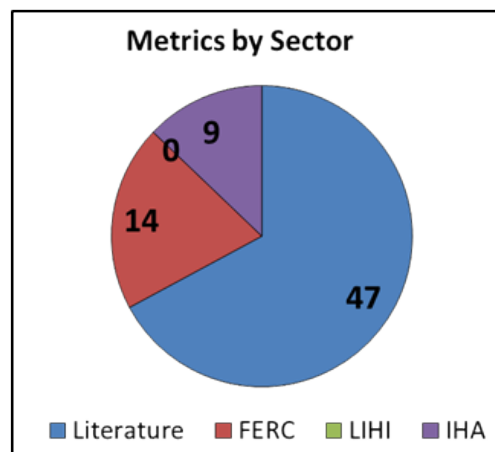

Metrics by Type

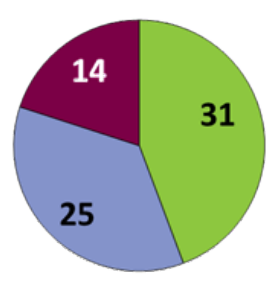

$\square$ Measure $\square$ Statistic

\section{GM_Sediment and} substrate
Sediment and substrate properties such as substrate particle size, bedload, sediment entrainment or deposition, bedrock composition

\begin{tabular}{|l|l|}
\hline \multicolumn{1}{|c|}{ Metric Type } & \multicolumn{1}{|c|}{ Metric Name } \\
\hline Measure & Bedload trans port \\
\hline Measure & Dry bulk density of sediment \\
\hline Measure & Excavation volume \\
\hline Measure & Gravel deposit area \\
\hline Measure & Gravel deposit depth \\
\hline Measure & Natural sediment \\
\hline Measure & Particle size analysis \\
\hline Measure & $\begin{array}{l}\text { Periodic ass ess ments of lake } \\
\text { sedimentation }\end{array}$ \\
\hline Measure & Reservoir sediment volume \\
\hline Measure & Sediment export \\
\hline Measure & Sediment grain size \\
\hline Measure & Sediment inflow \\
\hline Measure & Sediment layer depth and thickness \\
\hline Measure & Sediment type \\
\hline Measure & Specific degradation land \\
\hline Measure & Substrate composition qualitative \\
\hline Measure & Substrate size \\
\hline Measure & Substrate type \\
\hline Measure & Suspended sediment \\
\hline Statistic & GlS cells with deposition-- percent \\
\hline Statistic & GlS cells with scour-- percent \\
\hline Statistic & Minerological analysis \\
\hline Statistic & $\begin{array}{l}\text { Modeling of erosion and } \\
\text { sedimentation scenarios }\end{array}$ \\
\hline Statistic & Reservoir sediment trapping \\
\hline Statistic & Seservoir sediment yield \\
\hline Statistic & Sediment grain size \\
\hline Statistic & $\begin{array}{l}\text { Sediment grain size-- cumulative } \\
\text { frequency distribution }\end{array}$ \\
\hline Statistic & $\begin{array}{l}\text { Sediment grains below threshold-- } \\
\text { percent }\end{array}$ \\
\hline Statistic & Sediment inflow-- mean annual \\
\hline Statistic & Statistic \\
\hline
\end{tabular}

\begin{tabular}{|l|l|}
\hline \multicolumn{1}{|c|}{ Metric Type } & \multicolumn{1}{c|}{ Metric Name } \\
\hline Statistic & Sedimentation-- mean annual \\
\hline Statistic & Substrate coars eness regulated site \\
\hline Statistic & Substrate coars eness unregulated site \\
\hline Statistic & Substrate heterogeneity regulated site \\
\hline Statistic & $\begin{array}{l}\text { Substrate heterogeneity unregulated } \\
\text { site }\end{array}$ \\
\hline Statistic & Substrate particle size -- average \\
\hline Statistic & Substrate particle size -- median \\
\hline Statistic & Transect \& sieve analysis of sediment \\
\hline Indicator & Annual shoreline erosion inspections \\
\hline Indicator & Bathymetric monitoring of reservoir \\
\hline Indicator & Bedload trans port \\
\hline Indicator & Brune's design equations \\
\hline Indicator & $\begin{array}{l}\text { Erosion and subs equent sediment } \\
\text { tranport }\end{array}$ \\
\hline Indicator & Landslides and erosion \\
\hline Indicator & $\begin{array}{l}\text { Percent volume fraction of s ediment }< \\
\text { 8um in diameter }\end{array}$ \\
\hline Indicator & Reservoir sediment trapping efficiency \\
\hline Indicator & Sediment storage-- fine \\
\hline Indicator & Sediment supply \\
\hline Indicator & Sediment transport-- historic \\
\hline Indicator & Suspended sediment transport \\
\hline &
\end{tabular}

ORNL Catalog of Environmental Metrics for Hydropower - 32 


\subsection{LC_Land Cover}

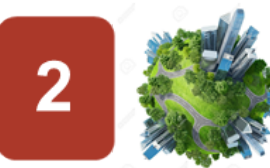

LC Land Cover

Land cover type (the type of physical material on the Earth's surface) is an important measure of ecosystem health because it influences many other environmental properties - ranging from river and floodplain sedimentation rates to fragmentation of habitats and wildlife populations-at scales ranging from site to landscape. Changes in land cover type are an important component of a full description of ecosystem changes associated with hydropower development, such as increases in wetted surface resulting from reservoir formation, or fragmentation of the surrounding landscape due to the installation of supporting infrastructure (e.g., transmission lines, roads). Land cover characterization before and after hydropower development is an important component of understanding hydropower-affected ecosystems.

\begin{tabular}{|c|c|c|}
\hline Sector & \# of studies & \# of metrics \\
\hline IHA & 4 & 4 \\
\hline LIIHI & 5 & 28 \\
\hline FERC & 5 & 50 \\
\hline $\begin{array}{l}\text { Peer-Reviewed } \\
\text { Literature }\end{array}$ & 247 & 40 \\
\hline Totals & 261 & 122 \\
\hline
\end{tabular}

Land cover parameter frequency at study-level (extraction table)

Primary

Category Parameter

Land cover LC_Area impacted, project area

LC_Flooplain or riparian vegetatio

LC_Land cover class

LC_Protected land

LC_Reservoir inundatio

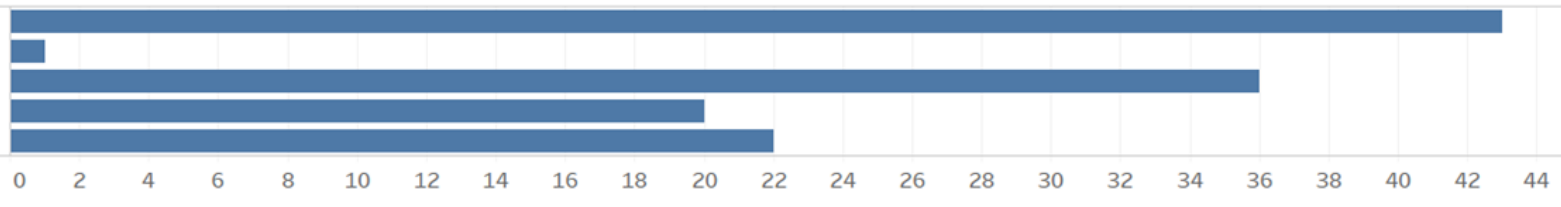

Count of Parameter for each Parameter broten down by Primary Gategory The view is flltered on Primary Gategory which keeps Land cover.

Count of Parameter

Spatial scales for land cover represented at study-level (extraction table)

Primary

Category Spatscale

Land cover Basin

Dam

Landscape

Project

Reservoir

River_downstream

River_upstream

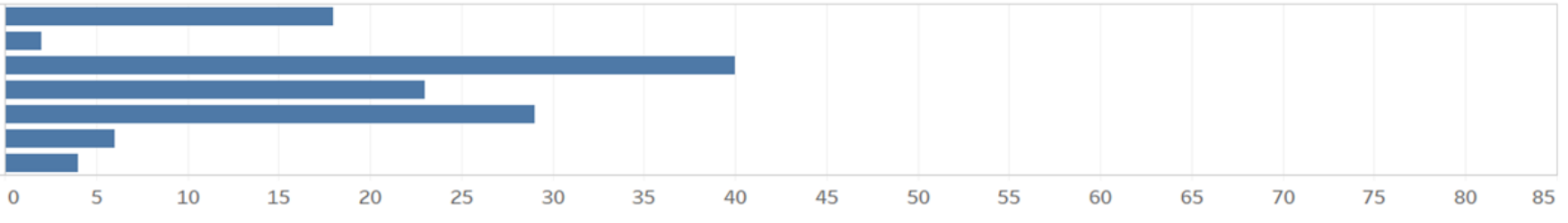

Count of Spatscale

ORNL Catalog of Environmental Metrics for Hydropower - 33 


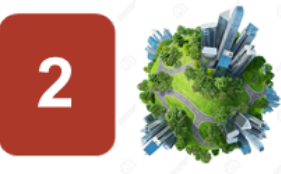

LC Land Cover
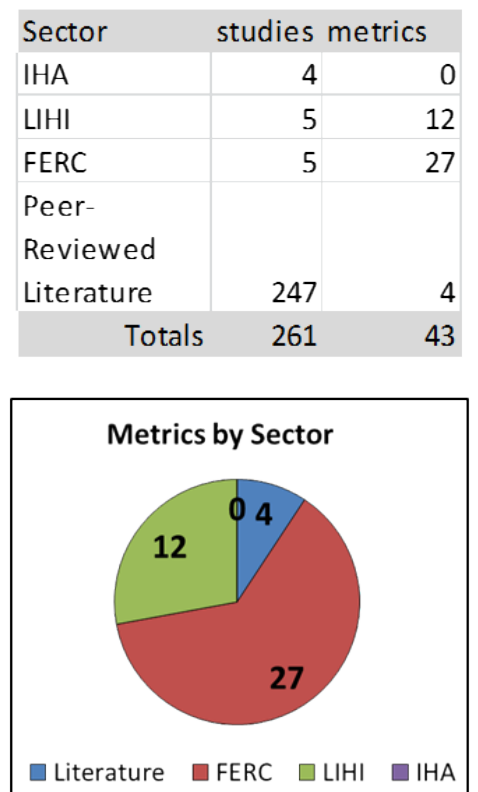

Metrics by Type

0

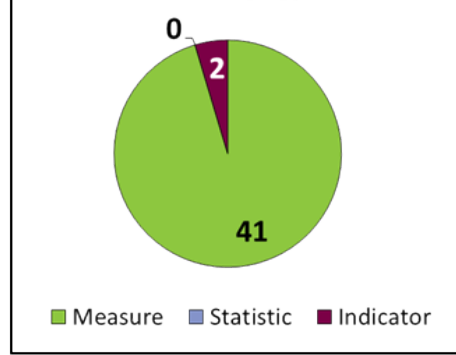

LC_Area impacted,

project area
Project boundary area, area impacted by the project as whole, not related to reservoir inundation or land cover

\begin{tabular}{|l|l|}
\hline \multicolumn{1}{|c|}{ Metric Type } & \multicolumn{1}{|c|}{ Metric Name } \\
\hline Measure & Bypass length \\
\hline Measure & Byproduct disposal area \\
\hline Measure & Dock permit/ access number \\
\hline Measure & Facility footprint \\
\hline Measure & Indian reservation presence \\
\hline Measure & Length dewatered river \\
\hline Measure & Non-reservoir facilities area \\
\hline Measure & Patch area \\
\hline Measure & Patch number \\
\hline Measure & Potential effect area \\
\hline Measure & Project boundary area \\
\hline Measure & Project boundary area-- land \\
\hline Measure & Project boundary area-- water \\
\hline Measure & Recreational land area \\
\hline Measure & Reservoir buffer zone acreage \\
\hline Measure & Reservoir buffer zone area \\
\hline Measure & Reservoir residences number \\
\hline Measure & River length al tered flow \\
\hline Measure & Stream bed impact area \\
\hline Measure & Tailwater length \\
\hline Measure & Transmission line length \\
\hline Measure & Transport distance of materials \\
\hline Measure & Upland forest area impacted \\
\hline Measure & $\begin{array}{l}\text { Upland forest habitat area } \\
\text { impacted }\end{array}$ \\
\hline Measure & Wetland habitat area impacted \\
\hline Indicator & Proximity index \\
\hline Indicator & Wetland area impacted-- deviation \\
\hline
\end{tabular}

ORNL Catalog of Environmental Metrics for Hydropower - 34 


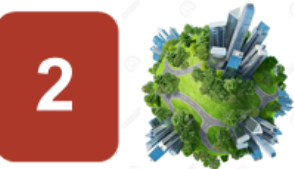

LC Land Cover

\begin{tabular}{|l|r|r|}
\hline Sector & studies metrics \\
\hline IHA & 4 & 0 \\
\hline LIHI & 5 & 0 \\
\hline FERC & 5 & 1 \\
\hline Peer- & & \\
Reviewed & & \\
Literature & 247 & 0 \\
\hline \multicolumn{1}{|r|}{ Totals } & 261 & 1 \\
\hline
\end{tabular}

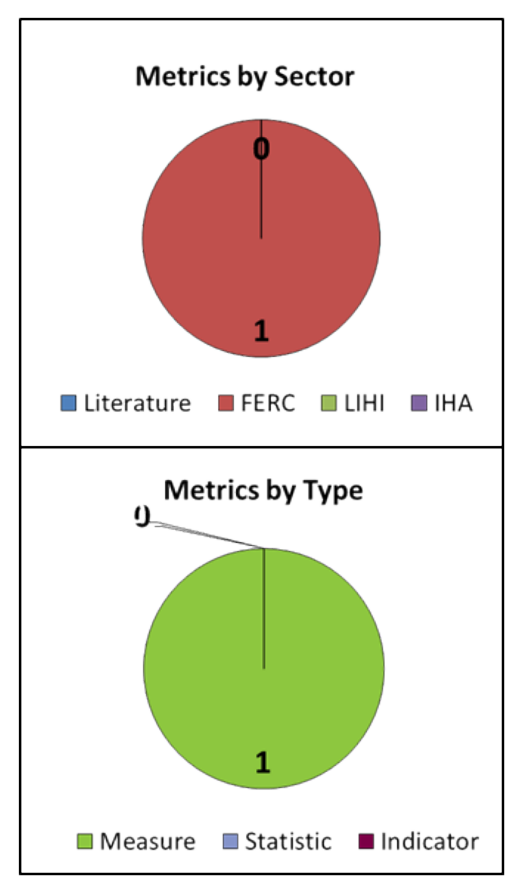

\section{LC_Floodplain or}

riparian vegetation
Properties of floodplain or riparian vegetation such as riparian

encroachment or floodplain area

\begin{tabular}{|l|c|}
\hline Metric Type & Metric Name \\
\hline Measure & River length of vegetation cover \\
\hline
\end{tabular}




\subsubsection{LC_Land cover class}

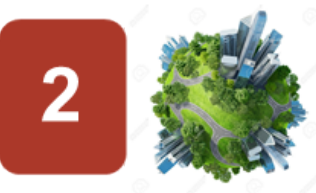

\section{LC Land Cover}

\begin{tabular}{|l|r|r|}
\hline Sector & studies metrics \\
\hline IHA & 4 & 4 \\
\hline LIHI & 5 & 0 \\
\hline FERC & 5 & 5 \\
\hline Peer- & & \\
Reviewed & & \\
Literature & 247 & 27 \\
\hline \multicolumn{1}{r|}{ Totals } & 261 & 36 \\
\hline
\end{tabular}

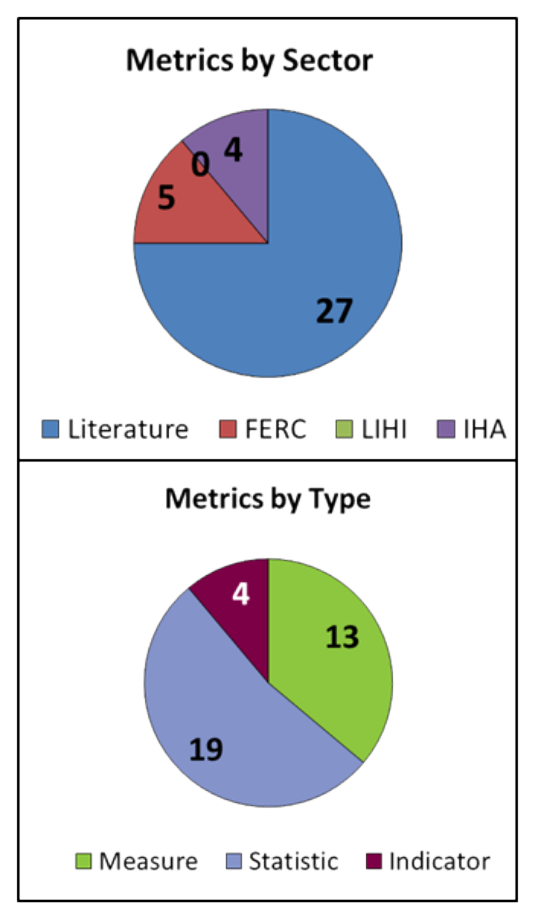

\section{LC_Land cover class}

\begin{tabular}{|l|l|}
\hline \multicolumn{1}{|c|}{ Metric Type } & \multicolumn{1}{|c|}{ Metric Name } \\
\hline Measure & $\begin{array}{l}\text { Fluvial marsh area large regulated } \\
\text { tributaries }\end{array}$ \\
\hline Measure & $\begin{array}{l}\text { Fluvial marsh area large } \\
\text { unregulated tributaries }\end{array}$ \\
\hline Measure & Forest area \\
\hline Measure & Forest cover change \\
\hline Measure & Ground cover \\
\hline Measure & Lake area \\
\hline Measure & Land development class \\
\hline Measure & Land use \\
\hline Measure & Open land area \\
\hline Measure & Shoreline development length \\
\hline Measure & Vegetation type \\
\hline Measure & Wetland area \\
\hline Statistic & Deforestation modeling \\
\hline Statistic & Forest area-- percent \\
\hline Statistic & Human population density \\
\hline Statistic & Open land-- percent \\
\hline Statistic & Reservoir area-- percent \\
\hline Statistic & Shoreline developed-- percent \\
\hline Statistic & Shoreline forested-- percent \\
\hline Statistic & Watershed artificial land cover \\
\hline Statistic & Watershed bare land cover \\
\hline Statistic & Watershed coniferous land cover \\
\hline Statistic & Watershed cultivated land cover \\
\hline Statistic & Watershed deciduous land cover \\
\hline Statistic & Watershed herbaceous land cover \\
\hline Statistic & Watershed mixed forest land cover \\
\hline & \\
\hline & \\
\hline
\end{tabular}

Type of land cover, changes in land cover

\begin{tabular}{|l|l|}
\hline \multicolumn{1}{|c|}{ Metric Type } & \multicolumn{1}{c|}{ Metric Name } \\
\hline Statistic & Watershed snow/ice land cover \\
\hline Statistic & Watershed wetland land cover \\
\hline Statistic & Watershed wetted land cover \\
\hline Statistic & Wetland area-- percent \\
\hline Indicator & Baseline land use \\
\hline Indicator & Change in vegetation cover \\
\hline Indicator & Cover \\
\hline Indicator & $\begin{array}{l}\text { Satellite imagery analysis of land } \\
\text { use trends }\end{array}$ \\
\hline
\end{tabular}

Frequencies based on EMH Catalog Contents as of January 31, 2017

ORNL Catalog of Environmental Metrics for Hydropower - 36 


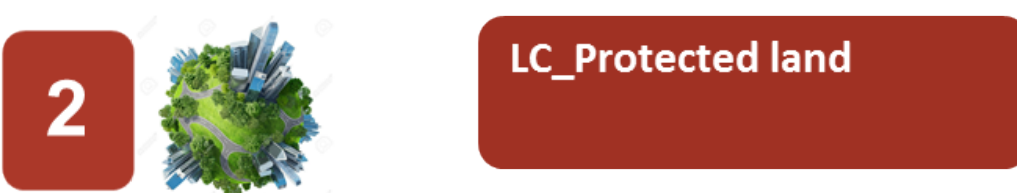

\section{LC Land Cover}

\begin{tabular}{|l|r|r|}
\hline Sector & studies metrics \\
\hline IHA & 4 & 0 \\
\hline LIHI & 5 & 8 \\
\hline FERC & 5 & 9 \\
\hline Peer- & & \\
Reviewed & & \\
Literature & 247 & 3 \\
\hline \multicolumn{1}{|r|}{ Totals } & 261 & 20 \\
\hline
\end{tabular}

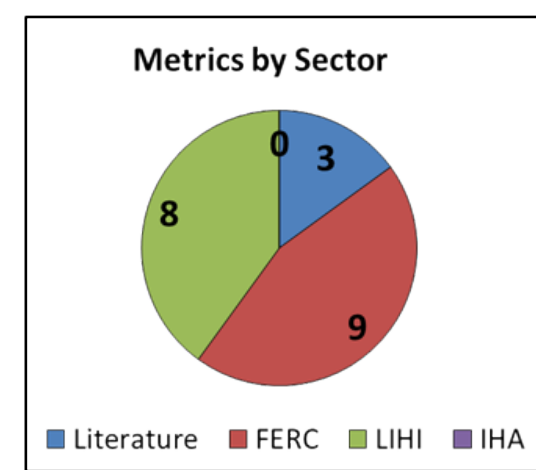

\begin{tabular}{|l|l|}
\hline \multicolumn{1}{|c|}{ type } & \multicolumn{1}{c|}{ MetricName } \\
\hline Measure & Conservation buffer zone presence \\
\hline Measure & Conservation land area \\
\hline Measure & Federal land area \\
\hline Measure & Federal land presence \\
\hline Measure & $\begin{array}{l}\text { Federal/conservation land } \\
\text { perimeter bounding }\end{array}$ \\
\hline Measure & Length shoreline protected \\
\hline Measure & Reservoir buffer zone area \\
\hline Measure & Reservoir buffer zone width \\
\hline Measure & Wildlife habitat lands \\
\hline Statistic & $\begin{array}{l}\text { Conservation land within reservoir } \\
\text { buffer-- percent }\end{array}$ \\
\hline Statistic & $\begin{array}{l}\text { Shoreline length forested-- } \\
\text { percent }\end{array}$ \\
\hline Statistic & $\begin{array}{l}\text { Shoreline protected/federal land-- } \\
\text { percent }\end{array}$ \\
\hline Indicator & $\begin{array}{l}\text { Payment for ecosystem services } \\
\text { related to forest management }\end{array}$ \\
\hline Indicator & $\begin{array}{l}\text { Value forest conservation for dam } \\
\text { operator }\end{array}$ \\
\hline Indicator & $\begin{array}{l}\text { Value forest conservation for } \\
\text { government }\end{array}$ \\
\hline
\end{tabular}

$\square$ Measure $\square$ Statistic $\square$ Indicator

ORNL Catalog of Environmental Metrics for Hydropowererengigs based on EMH Catalog Contents as of January 31, 2017 


\subsubsection{LC_Reservoir inundation}

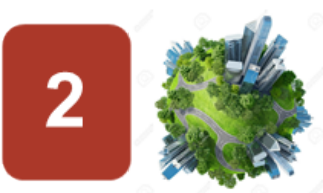

LC Land Cover

\begin{tabular}{|l|r|r|}
\hline Sector & studies metrics \\
\hline IHA & 4 & 0 \\
\hline LIHI & 5 & 8 \\
\hline FERC & 5 & 8 \\
\hline Peer- & & \\
Reviewed & & \\
Literature & 247 & 6 \\
\hline \multicolumn{1}{|r|}{ Totals } & 261 & 22 \\
\hline
\end{tabular}
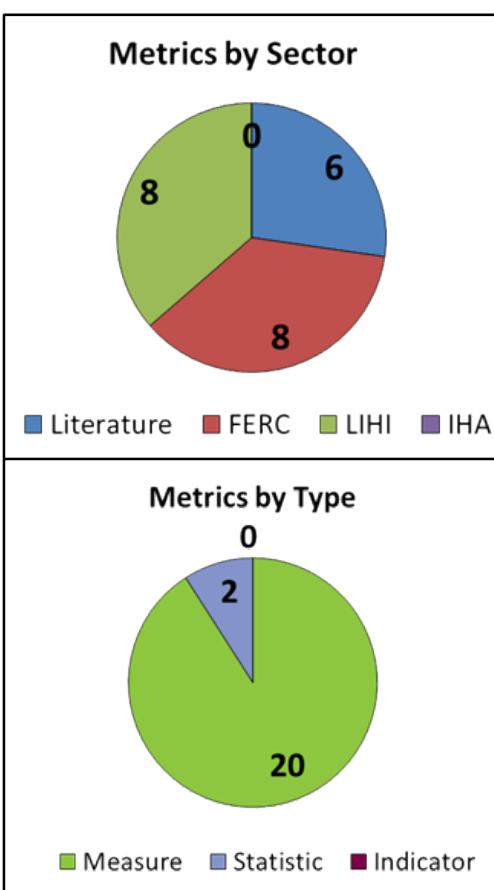

LC_Reservoir inundation

\begin{tabular}{|l|l|}
\hline \multicolumn{1}{|c|}{ Metric Type } & \multicolumn{1}{|c|}{ Metric Name } \\
\hline Measure & Island number \\
\hline Measure & Island size \\
\hline Measure & Normal full pond surface area \\
\hline Measure & Reservoir capacity area \\
\hline Measure & Reservoir inundation area \\
\hline Measure & Reservoir inundation length \\
\hline Measure & $\begin{array}{l}\text { Reservoir length at average } \\
\text { water level }\end{array}$ \\
\hline Measure & Reservoir surface area \\
\hline Measure & Shoreline length \\
\hline Measure & Shoreline perimeter \\
\hline Statistic & Reservoir length-- maximum \\
\hline Statistic & Reservoir width-- maximum \\
\hline
\end{tabular}

Frequencies based on EMH Catalog Contents as of January 31, 2017

ORNL Catalog of Environmental Metrics for Hydropower - 38 


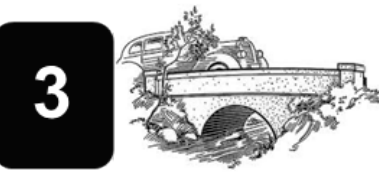

ID Infrastructure and Design

\begin{tabular}{|c|c|c|}
\hline Sector & \# of studies & $\#$ of metrics \\
\hline IHA & 4 & 6 \\
\hline LIIHI & 5 & 32 \\
\hline FERC & 5 & 77 \\
\hline Peer-Reviewed & & \\
\hline Literature & 247 & 21 \\
\hline Totals & 261 & 136 \\
\hline
\end{tabular}

Infrastructure parameter frequency at study-level (extraction table)

Primary

Category Parameter

Infrastructure ID_Dam attributes

design \& ID_Fish passage

development ID_Turbine

Hydropower infrastructure involves the construction of structures in-stream (for impounding water and generating power) and on adjacent riparian and terrestrial lands (for transmitting power and accessing the site). The selection of hydropower equipment, associated infrastructure, and management practices can bear directly and indirectly on the other six categories through a variety of factors. These include increased land cover fragmentation due to transmission lines, exposure of animals and humans to electromagnetic fields, changes in the volume and timing of water releases, and the use of industrial lubricants needed to keep hydropower turbines working properly.

Count of Parameter for each Parameter broken down by Primary Category. The view is filtered on Primary Category, which keeps Infrastructure design \& development.

Spatial scales for infrastructure represented at study-level (extraction table)

Primary

Category Spatscale

Infrastructure Basin

$\begin{array}{ll}\text { design \& } & \text { Dam } \\ \text { Landscape }\end{array}$

development Landscape

Project
Reach

Reservoir

River_downstream

Within dam

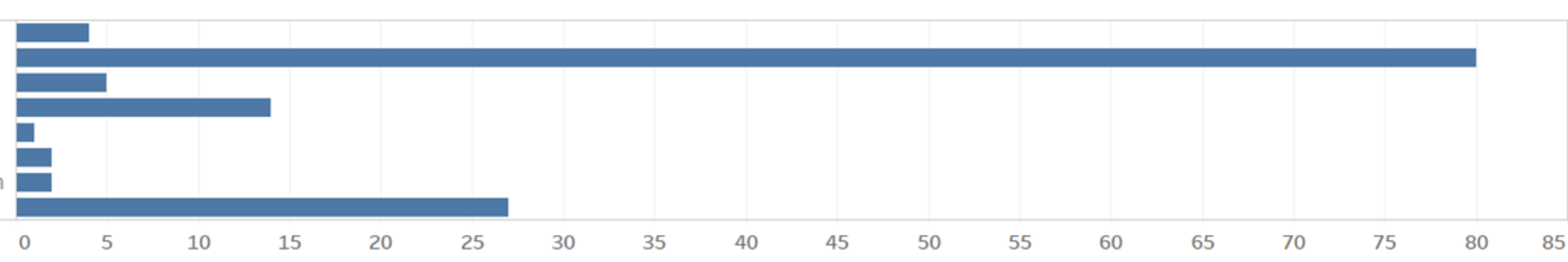

Count of Spatscale

ORNL Catalog of Environmental Metrics for Hydropower - 39 


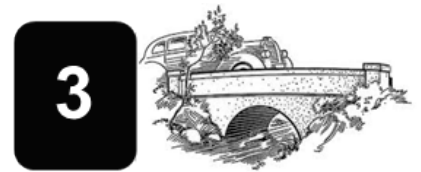

ID Infrastructure and Design

\begin{tabular}{|l|r|r|}
\hline Sector & studies & \multicolumn{2}{|c|}{ metrics } \\
\hline IHA & 4 & 3 \\
\hline LIHI & 5 & 28 \\
\hline FERC & 5 & 60 \\
\hline
\end{tabular}

Peer-

Reviewed

\begin{tabular}{crr} 
Literature & 247 & 13 \\
\hline Totals & 261 & 104
\end{tabular}

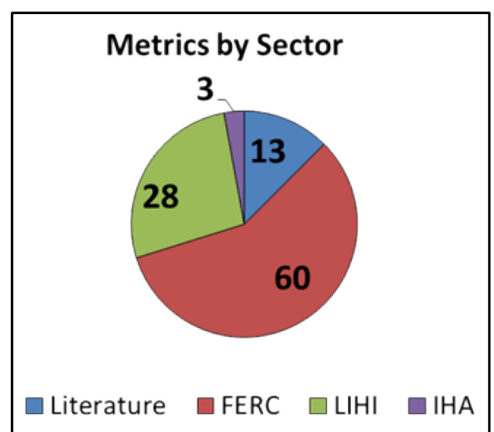

Metrics by Type

2

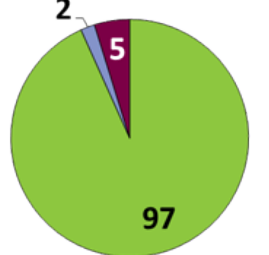

$\square$ Measure $\square$ Statistic

- Indicator

\section{ID_Dam attributes}

Head, dam height, spill gate type, bar rack, and so on

\begin{tabular}{|l|l|}
\hline \multicolumn{1}{|c|}{ Metric Type } & \multicolumn{1}{c|}{ Metric Name } \\
\hline Measure & Complex number \\
\hline Measure & Dam completion date \\
\hline Measure & Dam height \\
\hline Measure & Dam length \\
\hline Measure & Dam number \\
\hline Measure & Dam type \\
\hline Measure & Discharge in flume \\
\hline Measure & Discharge-- spillway \\
\hline Measure & Energy production \\
\hline Measure & Flashboard height \\
\hline Measure & Flashboard pres ence \\
\hline Measure & Flumelength \\
\hline Measure & Generation-- average annual \\
\hline Measure & Head \\
\hline Measure & Hydraulic head \\
\hline Measure & Increased capacity \\
\hline Measure & Increased hydraulic capacity \\
\hline Measure & Inflow design flood \\
\hline Measure & Installed capacity \\
\hline Measure & Intake depth \\
\hline Measure & Intake hydraulic capacity \\
\hline Measure & Intake presence \\
\hline Measure & Penstock length \\
\hline Measure & Penstock number \\
\hline Measure & Penstock/pipeline diameter \\
\hline Measure & Penstock/pi peline length \\
\hline Measure & Pipeline/tunnel/penstock length \\
\hline Measure & Power generation \\
\hline Measure & Powerhouse dimensions \\
\hline Measure & Powerhouse integral \\
\hline Measure & Powerhouse number \\
\hline Measure & Reservoir age \\
\hline Measure & Reservoir number \\
\hline Measure & Reservoir outflow lower outlet \\
\hline Measure & \\
\hline
\end{tabular}

\begin{tabular}{|l|l|}
\hline \multicolumn{1}{|c|}{ Metric Type } & \multicolumn{1}{c|}{ Metric Name } \\
\hline Measure & Sluiceway type \\
\hline Measure & Spill gate di mensions \\
\hline Measure & Spill gate number \\
\hline Measure & Spill gate type \\
\hline Measure & Spillway discharge capacity \\
\hline Measure & Spillway length \\
\hline Measure & Spillway pres ence \\
\hline Measure & Track rack dimensions \\
\hline Measure & Trash rack gate presence \\
\hline Measure & Trashrack presence \\
\hline Measure & Tunnel bypass length \\
\hline Measure & Tunnel length \\
\hline Measure & Tunnel number \\
\hline Measure & Turbine-generator aeration \\
\hline Measure & Turbine-generator unit number \\
\hline Measure & Wall height \\
\hline Measure & Water depth flume \\
\hline Measure & Water release depth qualitative \\
\hline Statistic & Open weir in advance of high flow \\
\hline Statistic & Percent of time firm power demand \\
& met \\
\hline Indicator & Dam-break envelope attenuation \\
\hline Indicator & Increased capacity \\
\hline Indicator & Increased generation \\
\hline Indicator & Sediment-transport through des ander \\
\hline Indicator & Wet season flow \\
\hline & \\
\hline
\end{tabular}

ORNL Catalog of Environmental Metrics for Hydropower - 40 


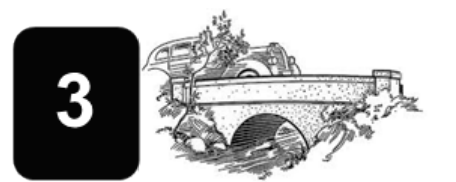

\section{ID_Fish passage}

Characteristics of fish passage structures such as slope, velocity, and discharge

ID Infrastructure and Design

\begin{tabular}{|c|c|c|}
\hline Sector & studies & metrics \\
\hline IHA & 4 & 2 \\
\hline LIHI & 5 & 3 \\
\hline FERC & 5 & 6 \\
\hline $\begin{array}{l}\text { Peer- } \\
\text { Reviewed }\end{array}$ & & \\
\hline Literature & 247 & 0 \\
\hline Totals & 261 & 11 \\
\hline
\end{tabular}

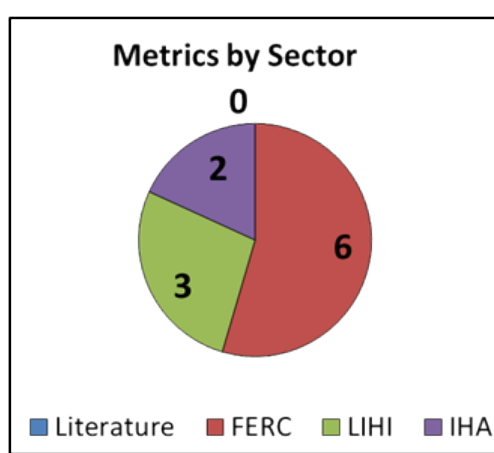

\begin{tabular}{|l|l|}
\hline \multicolumn{1}{|c|}{ Metric Type } & \multicolumn{1}{c|}{ Metric Name } \\
\hline Measure & Design population \\
\hline Measure & $\begin{array}{l}\text { Design population diadromous } \\
\text { fish }\end{array}$ \\
\hline Measure & Fish lift height \\
\hline Measure & Fish passage facility presence \\
\hline Measure & Fishway biomass capacity \\
\hline Measure & Trash rack spacing \\
\hline Indicator & Fish passage and hatchery \\
\hline Indicator & $\begin{array}{l}\text { Threshold water velocity at } \\
\text { intake }\end{array}$ \\
\hline
\end{tabular}

Literature $\quad$ FERC $\square \mathrm{LIHI} \square \mathrm{IHA}$ Metrics by Type

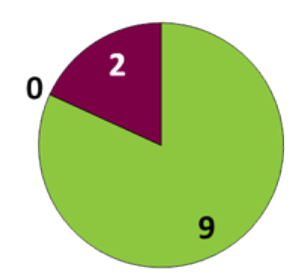

$\square$ Measure $\square$ Statistic $\quad$ Indicator 


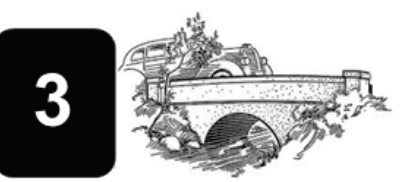

ID Infrastructure and Design

\begin{tabular}{|l|r|r|}
\hline Sector & studies & metrics \\
\hline IHA & 4 & 1 \\
\hline LIHI & 5 & 1 \\
\hline FERC & 5 & 11 \\
\hline \begin{tabular}{l|} 
Peer- \\
Reviewed \\
Literature
\end{tabular} & & \\
\hline \multicolumn{1}{|r|}{ Totals } & 247 & 8 \\
\hline
\end{tabular}

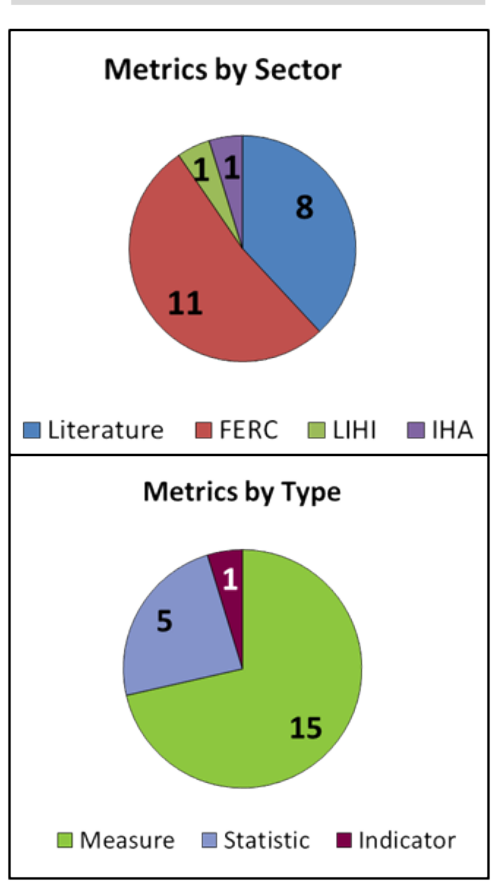

\section{ID_Turbine}

\begin{tabular}{|l|l|}
\hline \multicolumn{1}{|c|}{ type } & \multicolumn{1}{c|}{ MetricName } \\
\hline Measure & Approach velocity \\
\hline Measure & Dam hydraulic capacity \\
\hline Measure & Discharge-- turbine \\
\hline Measure & Sweep velocity \\
\hline Measure & Turbine hydraulic capacity \\
\hline Measure & Turbine type \\
\hline Measure & Turbine-generator aeration \\
\hline Measure & $\begin{array}{l}\text { Turbine-generator hydraulic } \\
\text { capacity }\end{array}$ \\
\hline Measure & Turbine-generator unit number \\
\hline Statistic & Approach velocity-- average \\
\hline Statistic & Percent of velocities $>0.12 \mathrm{~m} / \mathrm{s}$ \\
\hline Statistic & $\begin{array}{l}\text { Root mean square of approach } \\
\text { velocity fluctuations }\end{array}$ \\
\hline Statistic & Sweep velocity-- average \\
\hline Statistic & $\begin{array}{l}\text { Sweep velocity to approach } \\
\text { velocity ratio }\end{array}$ \\
\hline Indicator & Turbine abrasion \\
\hline
\end{tabular}
such as pressure, shear, cavitation, turbine type, turbine speed, blade strike

ORNL Catalog of Environmental Metrics for Hydropower - 42 


\subsection{CF_Connectivity and Fragmentation}

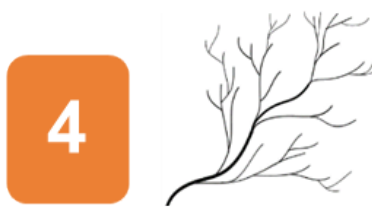

CF Connectivity

and

Fragmentation

\begin{tabular}{|l|r|r|}
\hline Sector & \# of studies & \# of metrics \\
\hline IHA & 4 & 5 \\
\hline LIHI & 5 & 25 \\
\hline FERC & 5 & 29 \\
\hline $\begin{array}{l}\text { Peer-Reviewed } \\
\text { Literature }\end{array}$ & 247 & 158 \\
\hline \multicolumn{1}{|r|}{ Totals } & 261 & 217 \\
\hline
\end{tabular}

Ecosystem connectivity (the degree to which a land cover type or ecosystem maintains continuity) and fragmentation (the degree to which an ecosystem or land cover type is disconnected) can affect the habitat quantity and quality for organisms in an ecosystem. Dams and their associated infrastructure can disrupt aquatic, riparian, and terrestrial connectivity, as well as groundwater connectivity, all of which can directly impact biota. Quantifying connectivity changes is therefore important for a full accounting of the environmental effects of hydropower.

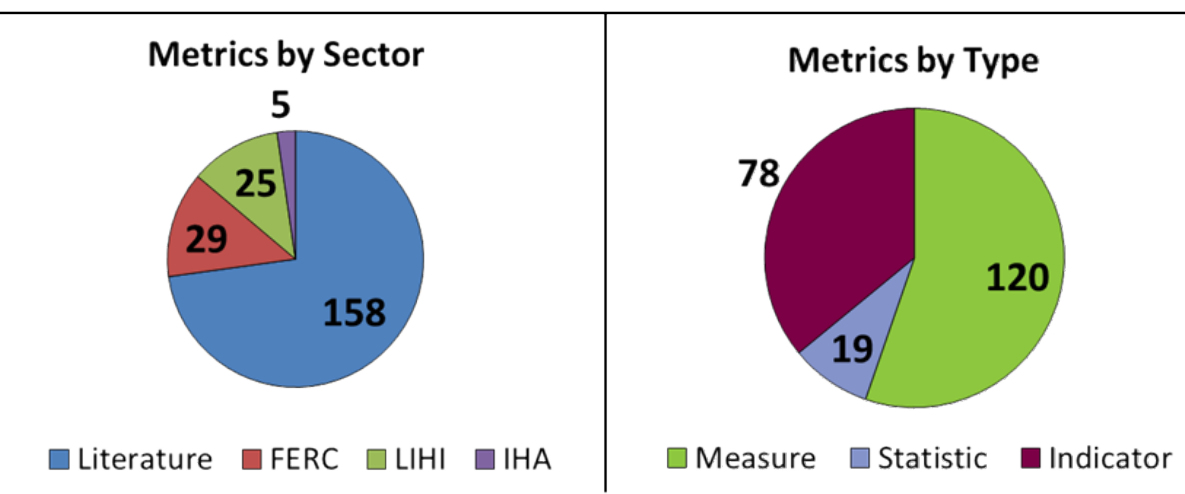

Connectivity parameter frequency at study-level (extraction table)

Primary

Category Parameter

Connectivity/ CF_Basin Area

fragmentation CF_Dendritic network and riverscape CF_Fish passage

$\begin{array}{lllllllllllllllllll}0 & 10 & 20 & 30 & 40 & 50 & 60 & 70 & 80 & 90 & 100 & 110 & 120 & 130 & 140 & 150 & 160 & 170 & 180\end{array}$ Count of Parameter

Count of Parameter for each Parameter broken down by Primary Category The view is filtered on Primary Category, which keeps Connectivity/fragmentation

Spatial scales for connectivity represented at study-level (extraction table)
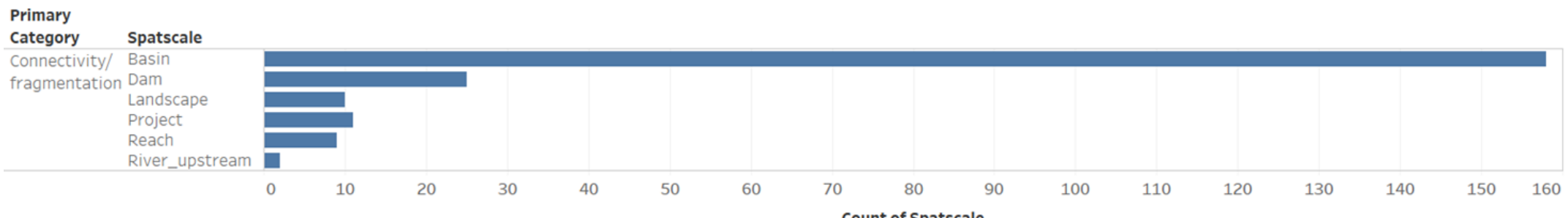

ORNL Catalog of Environmental Metrics for Hydropower - 43 


\subsubsection{CF_Basin Area}

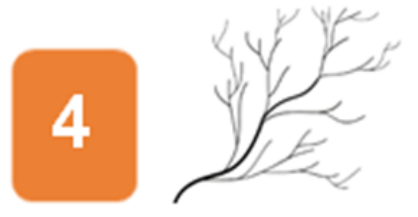

CF Connectivity

and

\section{Fragmentation}

\begin{tabular}{|c|c|c|}
\hline Sector & studies & metrics \\
\hline IHA & 4 & 0 \\
\hline LIHI & 5 & 2 \\
\hline FERC & 5 & 2 \\
\hline Peer-Reviewed & & \\
\hline Literature & 247 & 5 \\
\hline Totals & 261 & 9 \\
\hline
\end{tabular}

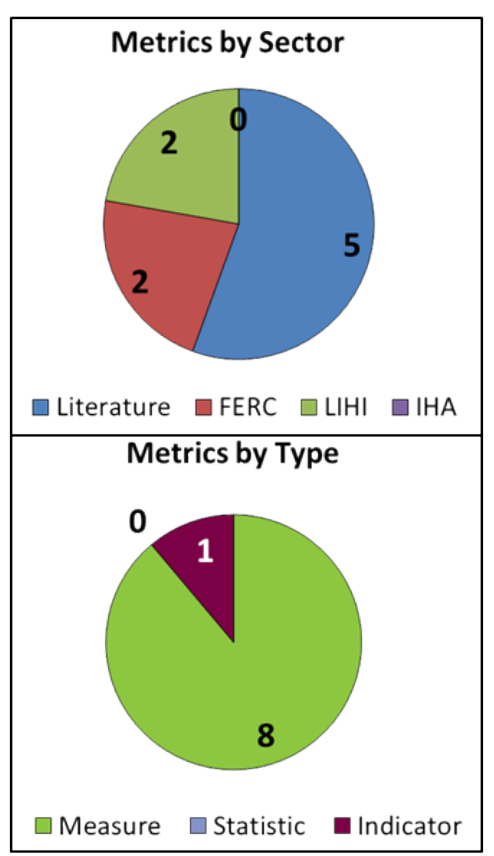

\section{CF_Basin area}

\begin{tabular}{|c|c|}
\hline Metric Type & Metric Name \\
\hline Measure & $\begin{array}{l}\text { Annual flow coefficient of } \\
\text { variation }\end{array}$ \\
\hline Measure & Catchment area \\
\hline Measure & $\begin{array}{l}\text { Percent difference in pre-dam } \\
\text { and post-dam annual flow } \\
\text { coefficient of variation }\end{array}$ \\
\hline Measure & River drainage area \\
\hline Indicator & $\begin{array}{l}\text { Percent difference between } \\
\text { drainage area at flow-regulated } \\
\text { and free-flowing fish survey } \\
\text { pairs (calculated as [(reference) } \\
\text { dam) } / \text { dam] } \cdot 100 \text { ) a }\end{array}$ \\
\hline
\end{tabular}

\section{Some aspect of area of river basin}

ORNL Catalog of Environmental Metrics for Hydropower - 44 


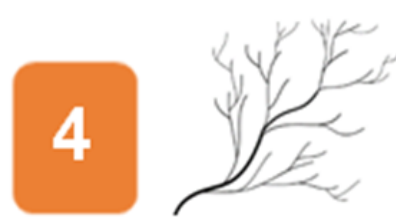

CF Connectivity

and

Fragmentation

\begin{tabular}{|l|r|r|}
\hline Sector & studies metrics \\
\hline IHA & 4 & 1 \\
\hline LIHI & 5 & 12 \\
\hline FERC & 5 & 11 \\
\hline \begin{tabular}{l|r|} 
Peer-Reviewed \\
Literature
\end{tabular} \\
\hline \multicolumn{1}{|r|}{ Totals } & 247 & 150 \\
\hline
\end{tabular}

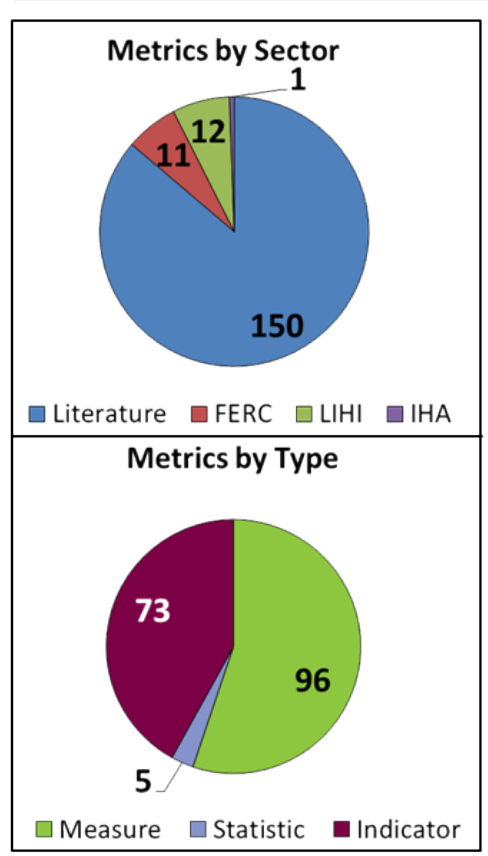

CF_Dendritic network and riverscape

\begin{tabular}{|l|l|}
\hline Metric Type & \multicolumn{1}{|c|}{ Metric Name } \\
\hline Measure & Distance from dam \\
\hline Measure & Distance from estuary \\
\hline Measure & Distance from mouth \\
\hline Measure & Distance from river mouth \\
\hline Measure & Distance pre-/ post-dam survey \\
\hline Measure & Distance to source \\
\hline Measure & Length 1st order upstream \\
\hline Measure & Length 2nd order upstream \\
\hline Measure & Length 3rd order upstream \\
\hline Measure & Length 4th order upstream \\
\hline Measure & Length river fragments \\
\hline Measure & Length river network \\
\hline Measure & Length river upstream \\
\hline Measure & Linear distance between al tered \\
& and unaltered sites \\
\hline Measure & Number barriers \\
\hline Measure & Number barriers downstream \\
\hline Measure & Number barriers upstream \\
\hline Measure & Number existing dams \\
\hline Measure & Number facilities in basin \\
\hline Measure & Number natural barriers \\
\hline Measure & Number new dams \\
\hline Measure & Number other dams \\
\hline Measure & Number reservoir tributaries \\
\hline Measure & Number river fragments \\
\hline Measure & River distance between dams \\
\hline Measure & River mile \\
\hline Measure & Total river length \\
\hline Measure & Total river water volume fragment \\
\hline
\end{tabular}

Fragment length, dendritic connectivity index, barrier index, river distance between dams and projects

\begin{tabular}{|l|l|}
\hline Metric Type & \multicolumn{1}{|c|}{ Metric Name } \\
\hline Measure & $\begin{array}{l}\text { Total water volume entire river } \\
\text { network }\end{array}$ \\
\hline Measure & Upstream habitat length \\
\hline Statistic & $\begin{array}{l}\text { \%total river length 1st order } \\
\text { upstream }\end{array}$ \\
\hline Statistic & $\begin{array}{l}\text { \%total river length 2nd order } \\
\text { upstream }\end{array}$ \\
\hline Statistic & $\begin{array}{l}\text { \%total river length 3rd order } \\
\text { upstream }\end{array}$ \\
\hline Statistic & Distance from confluence \\
\hline Statistic & $\begin{array}{l}\text { Maximum distance between any } \\
\text { two components (gauge(s), fish } \\
\text { survey and dam) }\end{array}$ \\
\hline Indicator & Dam permeability by aquatic biota \\
\hline Indicator & Degree of regulation \\
\hline Indicator & Dendritic connectivity index \\
\hline Indicator & Ensured tribuatary connectivity \\
\hline Indicator & $\begin{array}{l}\text { Percent difference drainage area } \\
\text { between regulated and } \\
\text { unregulated area }\end{array}$ \\
\hline Indicator & River connectivity index-- fish \\
\hline Indicator & $\begin{array}{l}\text { River connectivity index } \\
\text { disconnections }\end{array}$ \\
\hline Indies based on EMH Catalog Contents as of January 31,2017
\end{tabular}

ORNL Catalog of Environmental Metrics for Hydropower - 45 


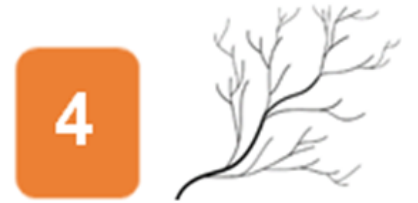

CF Connectivity

and

\section{Fragmentation}

\begin{tabular}{|l|r|r|}
\hline Sector & studies metrics \\
\hline IHA & 4 & 4 \\
\hline LIHI & 5 & 11 \\
\hline FERC & 5 & 16 \\
\hline \begin{tabular}{l|r|} 
Peer-Reviewed \\
Literature
\end{tabular} \\
\multicolumn{1}{|l|}{ Totals } & 247 & 3 \\
\hline
\end{tabular}

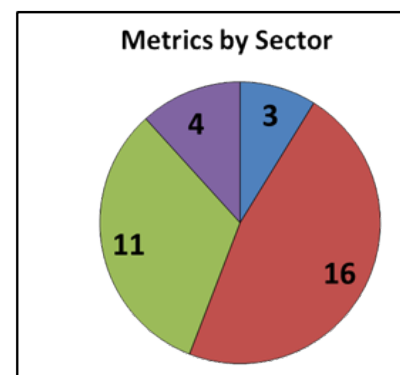

$\square$ Literature $\square$ FERC $\square$ LIHI $\square$ IHA Metrics by Type

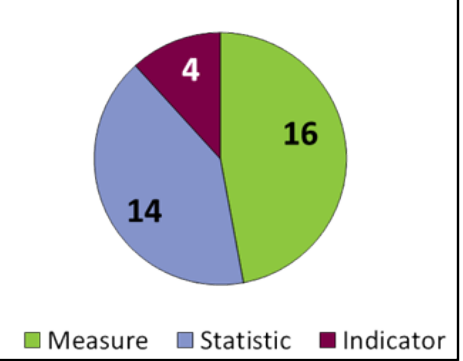

CF_Fish passage

Mitigated fish passage, including presence of upstream or downstream passage or length of bypass

\begin{tabular}{|l|l|}
\hline Metric Type & \multicolumn{1}{|c|}{ Metric Name } \\
\hline Measure & Entrainment/impingment presence \\
\hline Measure & Fish passage abundance \\
\hline Measure & Fish passage presence-- diadromous \\
\hline Measure & Fish passage presence-- non-native \\
\hline Measure & Fish passage presence-- potamodromous \\
\hline Measure & Fish passage success-- count \\
\hline Measure & Fishway biomass capacity \\
\hline Measure & Number fish successfully passing dam \\
\hline Measure & Number of elvers navigating the elver \\
& ladder \\
\hline Measure & Turbine passage mortality \\
\hline Statistic & Downstream passage survival-- minimum \\
\hline Statistic & Fish passage abundance mean \\
\hline Statistic & Fish passage abundance variation \\
\hline Statistic & Fish passage richness \\
\hline Statistic & Fish passage species composition \\
\hline Statistic & Fish passage success \\
\hline Statistic & Fish passage success-- average \\
\hline Statistic & Fish passage survival \\
\hline Statistic & Fishway genetic exchange \\
\hline Statistic & Number of emigrants \\
\hline Statistic & Passed fish-- maximum \\
\hline Statistic & Survival rate fish passage \\
\hline Statistic & Turbine passage survival \\
\hline Indicator & Fish passage and restocking \\
\hline Indicator & Fish passage efficiency \\
\hline Indicator & Flow verification at fish ladder \\
\hline Indicator & Rescued stranded fish \\
\hline
\end{tabular}

Indicator Rescued stranded fish

ORNL Catalog of Environmental Metrics for Hydropower - 46 


\subsection{W1_Water Quantity}

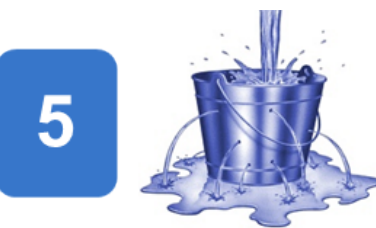

Water quantity refers to the amount of water found in-stream, in a reservoir, or in groundwater stores. It includes flow magnitude, duration, frequency, timing, and the rate of change of flows. Hydropower development can alter the quantity of water in several ways - through water storage in reservoirs, increased evaporation rates, changes in the availability of in-channel water downstream of a dam, and changes in the quantity of groundwater both upstream and downstream of dams. Because hydropower systems may be operated to fill a variety of purposes, changes to water quantity may occur at a variety of temporal scales ranging from diel to annual. Changes in water quantity can ultimately affect human and wildlife populations by altering water supplies for a variety of

W1 Water Quantity

end uses (e.g., agricultural, drinking, municipal, industrial, recreational) and by changing habitat availability.

\begin{tabular}{|c|c|c|}
\hline Sector & \# of studies & \# of metrics \\
\hline IHA & 4 & 14 \\
\hline LIIHI & 5 & 48 \\
\hline FERC & 5 & 344 \\
\hline Peer-Reviewed & & \\
\hline Literature & 247 & 628 \\
\hline Totals & 261 & 1034 \\
\hline
\end{tabular}

Water quantity parameter frequency at study-level (extraction table) Primary

Category Parameter

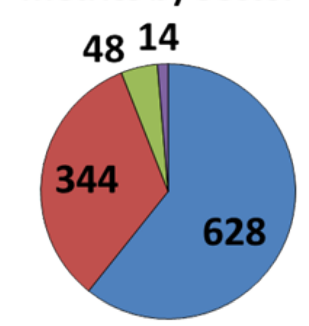

Metrics by Type

Paramet

HD_Basin attributes

HD_Diversion

HD_Reservoir hydrology

HD_Upstream regulation and inflow

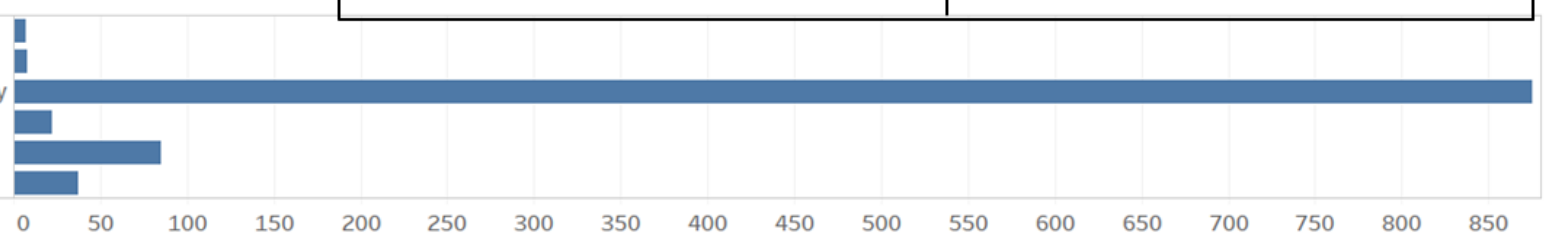

Count of Parameter

Count of Parameter for each Parameter broken down by Primary Category The vien is filtered on Primasy Cotegory, which keeps Water quantity.

Spatial scales for water quantity represented at study-level (extraction table)

Primary

Category Spatscale

Water Basin

quantity Dam

Landscap

Project

Reach

Reservoir

River_downstream

River_upstream

Within dam

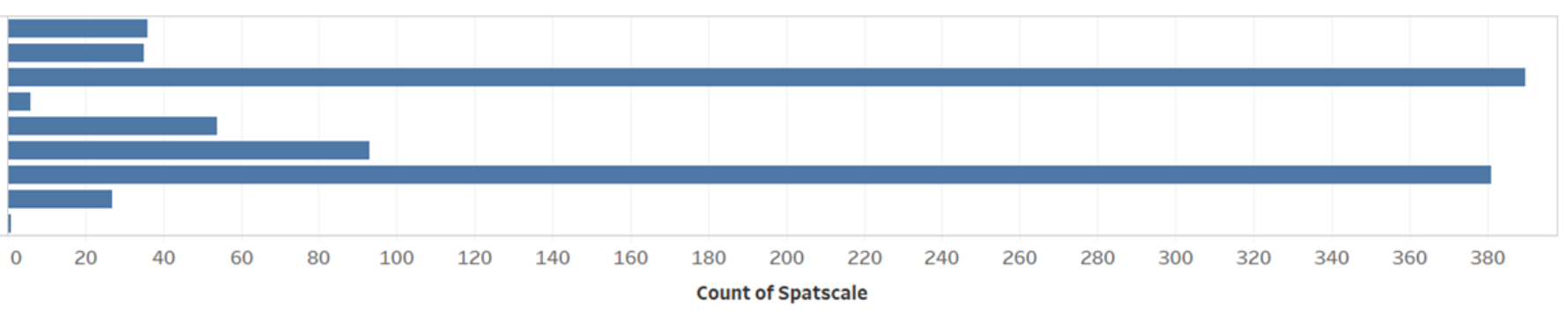

ORNL Catalog of Environmental Metrics for Hydropower - 47 


\subsubsection{W1_Basin Attributes}

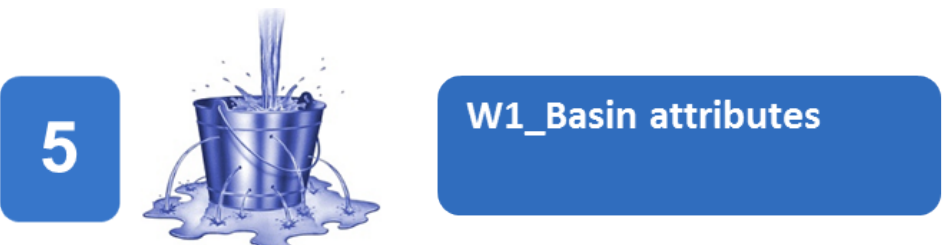

Attributes related to factors that influence hydrology (or were used in the context of hydrology), such as climate and precipitation

\section{W1 Water Quantity}

\begin{tabular}{|l|r|r|}
\hline Sector & studies metrics \\
\hline IHA & 4 & 4 \\
\hline LIHI & 5 & 0 \\
\hline FERC & 5 & 0 \\
\hline Peer- & & \\
Reviewed & & \\
Literature & 247 & 3 \\
\hline \multicolumn{1}{|r|}{ Totals } & 261 & 7 \\
\hline
\end{tabular}

\begin{tabular}{|l|l|}
\hline Metric Type & \multicolumn{1}{|c|}{ Metric Name } \\
\hline Measure & Precipitation \\
\hline Measure & Temperatur \\
\hline Statistic & Air temperature-- average \\
\hline Indicator & Baseline hydrology \\
\hline Indicator & Cataract Gorge flows review \\
\hline Indicator & Change in river morphology \\
\hline Indicator & River behavior \\
\hline
\end{tabular}

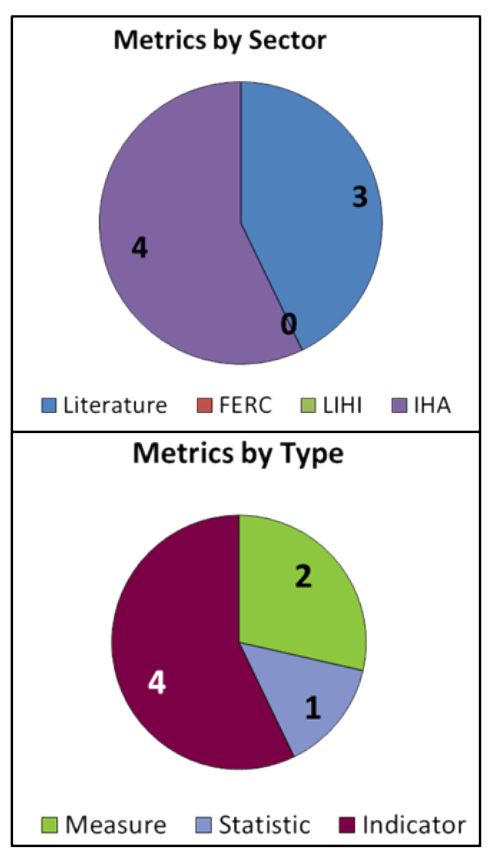




\subsubsection{W1_Diversion}

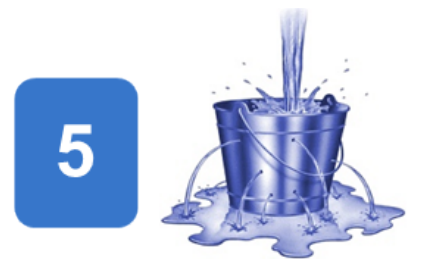

W1 Water Quantity

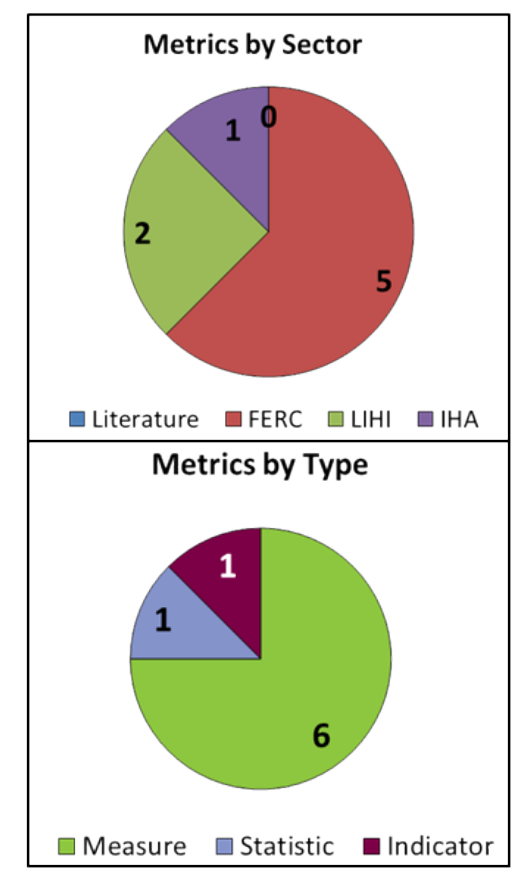

\begin{tabular}{|c|c|c|}
\hline Sector & studies & \\
\hline IHA & 4 & 1 \\
\hline LIHI & 5 & 2 \\
\hline FERC & 5 & 5 \\
\hline \multicolumn{3}{|l|}{ Peer- } \\
\hline Reviewed & & \\
\hline Literature & 247 & 0 \\
\hline Totals & 261 & 8 \\
\hline
\end{tabular}

\section{W1_Diversion}

\begin{tabular}{|l|l|}
\hline Metric Type & \multicolumn{1}{|c|}{ Metric Name } \\
\hline Measure & Bypass reach presence \\
\hline Measure & Consumptive withdrawal \\
\hline Measure & Diversion presence \\
\hline Statistic & Social water-use analysis \\
\hline Indicator & Discharge-- flow reduction bypass \\
\hline
\end{tabular}

\section{W_Diversion}

Quantitative properties of diversions such as volume or discharge of diversion or water for other uses 


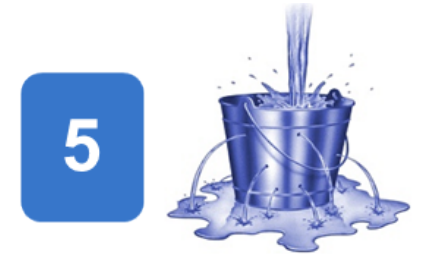

W1 Water Quantity
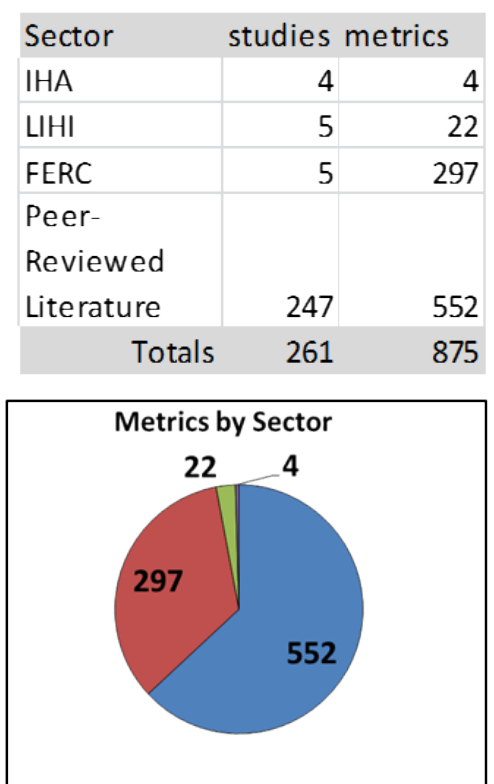

घiterature $\square$ FERC $\square$ LIHI $\square$ IHA

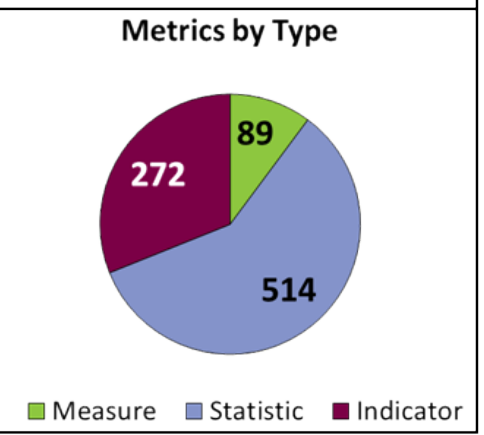

W1_Downstream discharge and hydrology
Measures that describe the magnitude, frequency, duration, periodicity, and timing of flows downstream of a hydropower facility, including changes to these characteristics

\begin{tabular}{|l|l|}
\hline Metric Type & \multicolumn{1}{|c|}{ Metric Name } \\
\hline Measure & Bankful discharge \\
\hline Measure & Boatable flow days \\
\hline Measure & Capacity water flow regulation \\
\hline Measure & Date water was first rel eased from dam spillway \\
\hline Measure & Discharge \\
\hline Measure & Discharge-- attraction flow \\
\hline Measure & Discharge-- channel creation flows \\
\hline Measure & Discharge-- date high flow \\
\hline Measure & Discharge-- discharge \\
\hline Measure & Discharge-- frequency high flow \\
\hline Measure & Discharge-- instantaneous outflow \\
\hline Measure & Discharge-- Julian date maximum \\
\hline Measure & Discharge-- magnitude high flow \\
\hline Measure & Discharge-- minimum \\
\hline Measure & Discharge-- minimum bypass \\
\hline Measure & Discharge-- minimum seasonal \\
\hline Measure & Discharge-- minimum target \\
\hline Measure & Discharge of canal outflows \\
\hline Measure & Discharge-- outfl ow \\
\hline Measure & Discharge-- outfl ow fluctuation \\
\hline Measure & Discharge-- regulated outflow \\
\hline Measure & Discharge-- subdaily flow fluctuations \\
\hline Measure & Discharge-- threshold seasonal low pulse \\
\hline Measure & $\begin{array}{l}\text { Discharge-- timing of greatest variation annual } \\
\text { hydrograph }\end{array}$ \\
\hline Measure & Discharge-- total hourly \\
\hline Measure & Discharge-- variable minimum magnitude \\
\hline Measure & Energy gradient elevation \\
\hline Measure & Hydropeaking scheme \\
\hline Measure & Mean flow \\
\hline Measure & Mode of operation \\
\hline Measure & Monthly flow \\
\hline Measure & Number of days water released from dam spillway \\
\hline Measure & potamodromous fish presence \\
\hline Measure & Spill Discharge \\
\hline
\end{tabular}

\begin{tabular}{|l|l|}
\hline Metric Type & \multicolumn{1}{|c|}{ Metric Name } \\
\hline Measure & Spill event magnitude \\
\hline Measure & Stream channel water depth \\
\hline Measure & Tailwater stage height \\
\hline Measure & Total discharge \\
\hline Measure & Total spill water volume-- seasonal \\
\hline Measure & Volumetric flow-- daily \\
\hline Measure & Water el evation downstream of dam \\
\hline Statistic & Bas eflow index \\
\hline Statistic & Catastrophic flood discharge \\
\hline Statistic & Change discharge--- standardized percent \\
\hline Statistic & Dam seepage discharge \\
\hline Statistic & Discharge-- coefficient of variation rate and \\
& frequency of change in conditions \\
\hline Statistic & Discharge-- minimum annual 3 day maximum \\
\hline Statistic & Discharge-- 1 day maximum \\
\hline Statistic & Discharge-- 1 day mi nimum \\
\hline Statistic & Discharge-- 3 day maximum \\
\hline Statistic & Discharge-- 30 day maximum \\
\hline Statistic & Discharge-- 30 day mi ni mum \\
\hline Statistic & Discharge-- 7 day maximum \\
\hline Statistic & Discharge-- 90 day maximum \\
\hline Statistic & Discharge-- annual \\
\hline Statistic & Discharge-- annual 1 day maximum \\
\hline Statistic & Discharge-- annual 1 day minimum \\
\hline Statistic & Discharge-- annual 3 day maximum \\
\hline Statistic & Discharge-- annual 3 day minimum \\
\hline Statistic & Discharge-- annual 30 day maximum \\
\hline Statistic & Discharge-- annual 30 day minimum \\
\hline Statistic & Discharge-- annual 7 day maximum \\
\hline Statistic & Discharge-- annual 7 day minimum \\
\hline Statistic & Discharge-- annual 90 day maximum \\
\hline Statistic & Discharge-- annual 90 day minimum \\
\hline Statistic & Discharge-- annual coefficient of variation \\
\hline Statistic & Discharge-- annual maximum \\
\hline Statistic & Discharge-- average annual \\
\hline
\end{tabular}

ORNL Catalog of Environmental Metrics for Hydropower - 50 


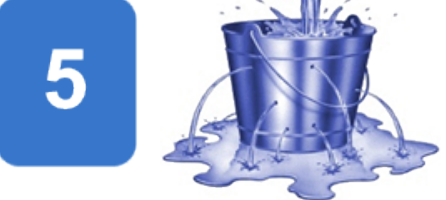

W1 Water Quantity

\begin{tabular}{|l|l|}
\hline Metric Type & \multicolumn{1}{|c|}{ Metric Name } \\
\hline Statistic & $\begin{array}{l}\text { Discharge-- average annual during } \\
\text { incubation period }\end{array}$ \\
\hline Statistic & Discharge-- average annual flow previous \\
\hline Statistic & Discharge-- average April \\
\hline Sta tistic & Discharge-- average August \\
\hline Statistic & Discharge-- average daily \\
\hline Statistic & Discharge-- average daily first year \\
\hline Statistic & Discharge-- average daily gate discharge \\
\hline Statistic & Discharge-- average daily turbine discharge \\
\hline Statistic & Discharge-- average December \\
\hline Statistic & Discharge-- average duration high pulses \\
\hline Statistic & Discharge-- average duration low pulses \\
\hline Statistic & Discharge-- average during rearing period \\
\hline Statistic & Discharge-- average during spawning period \\
\hline Statistic & Discharge-- average February \\
\hline Statistic & Discharge-- average hourly \\
\hline Statistic & Discharge-- average January \\
\hline Statistic & Discharge-- average July \\
\hline Statistic & Discharge-- average June \\
\hline Statistic & Discharge-- average March \\
\hline Statistic & Discharge-- average May \\
\hline Statistic & Discharge-- average monthly \\
\hline Statistic & Discharge-- average monthly dry year \\
\hline Statistic & $\begin{array}{l}\text { Discharge-- average monthly median } \\
\text { precipitation year }\end{array}$ \\
\hline Statistic & Discharge-- average November \\
\hline Statistic & Discharge-- average October \\
\hline Statistic & $\begin{array}{l}\text { Discharge-- average percent total dam } \\
\text { discharge released from spillway }\end{array}$ \\
\hline Statistic & $\begin{array}{l}\text { Discharge-- average score frequency and } \\
\text { duration of high and low pulses }\end{array}$ \\
\hline dtatistic & $\begin{array}{l}\text { Discharge-- average score magnitude and } \\
\text { duration of annual extremes }\end{array}$ \\
\hline
\end{tabular}

W1_Downstream discharge and hydrology
Measures that describe the magnitude, frequency, duration, periodicity, and timing of flows downstream of a hydropower facility, including changes to these characteristics

\begin{tabular}{|c|c|}
\hline Metric Type & Metric Name \\
\hline Statistic & $\begin{array}{l}\text { Discharge-- average score magnitude of } \\
\text { monthly water conditions }\end{array}$ \\
\hline Statistic & $\begin{array}{l}\text { Discharge-- average score rate and frequency } \\
\text { of change in conditions }\end{array}$ \\
\hline Statistic & Discharge-- average score timing of annual \\
\hline Statistic & Discharge-- average September \\
\hline Statistic & Discharge-- baseflowindex \\
\hline Statistic & Discharge change from the previous day \\
\hline Statistic & $\begin{array}{l}\text { Discharge-- coefficient of variation frequency } \\
\text { and duration high and low-flow pulses }\end{array}$ \\
\hline Statistic & $\begin{array}{l}\text { Discharge-- coefficient of variation } \\
\text { magnitude and duration of a nnual extremes }\end{array}$ \\
\hline Statistic & $\begin{array}{l}\text { Discharge-- coefficient of variation } \\
\text { magnitude of monthly water conditions }\end{array}$ \\
\hline Statistic & Discharge-- daily \\
\hline Statistic & Discharge-- daily annual maximum \\
\hline Statistic & Discharge-- daily maximum \\
\hline Statistic & Discharge-- days flow exceeds $25 \%$ first year \\
\hline Statistic & $\begin{array}{l}\text { Discharge-- days flow exceeds } 25 \% \\
\text { incubation period records }\end{array}$ \\
\hline Statistic & $\begin{array}{l}\text { Discharge-- days flow exceeds } 25 \% \text { previous } \\
\text { year flow }\end{array}$ \\
\hline Statistic & Discharge-- days flow exceeds $75 \%$ first year \\
\hline Statistic & $\begin{array}{l}\text { Discharge-- days flow exceeds } 75 \% \\
\text { incubation period records }\end{array}$ \\
\hline Statistic & $\begin{array}{l}\text { Discharge-- days flow exceeds } 75 \% \text { previous } \\
\text { year flow }\end{array}$ \\
\hline Statistic & Discharge-- days since high-flow \\
\hline Statistic & Discharge-- days since low-flow \\
\hline Statistic & Discharge-- days since reversal \\
\hline Statistic & Discha rge-- duration high-flow \\
\hline Statistic & Discharge-- duration Iow-flow \\
\hline Statistic & Discharge-- fall rate \\
\hline Statistic & Discharge flux index \\
\hline $\begin{array}{l}\text { Stptistifalog } \\
\text { Statistic }\end{array}$ & 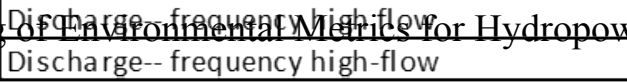 \\
\hline
\end{tabular}

\begin{tabular}{|c|c|}
\hline Metric Type & Metric Name \\
\hline Statistic & Discharge--frequency high-flow pulse \\
\hline Statistic & Discharge--frequency low-flow \\
\hline Statistic & Discharge-- frequency low-flow pulse \\
\hline Statistic & Discharge-- frequency reversals \\
\hline Statistic & Discharge-- frequency spill high flow \\
\hline Statistic & Discharge-- frequency zero-flow days \\
\hline Statistic & Discharge-- high pulse duration \\
\hline Statistic & Discharge-- high-flow frequency \\
\hline Statistic & Discharge-- Julian date a nnual maximum \\
\hline Statistic & Discharge-- Julian date a nnual minimum \\
\hline Statistic & Discharge-- Julian date maximum \\
\hline Statistic & Discharge-- maximum \\
\hline Statistic & Discharge-- maximum 10 day during rea ring \\
\hline Sta tistic & Discharge-- maximum 10 day during \\
\hline Statistic & Discharge-- maximum annual \\
\hline Statistic & Discharge-- maximum a nnual 1 day \\
\hline Statistic & Discharge-- maximum a nnual 1 day \\
\hline Statistic & Discharge-- maximum annual 1 day minimum \\
\hline Sta tistic & Discharge-- maximum a nnual 3 day \\
\hline Sta tistic & Discharge-- maximum a nnual 3 day \\
\hline Statistic & Discharge-- maximum annual 3 day minimum \\
\hline Statistic & Discharge-- maximum a nnual 30 day \\
\hline Sta tistic & Discharge-- maximum a nnual 30 day \\
\hline Statistic & Discharge-- maximum a nnual 30 day \\
\hline Statistic & Discharge-- maximum a nnual 7 day \\
\hline Statistic & Discharge-- maximum a nnual 7 day \\
\hline Statistic & Discharge-- maximum a nnual 7 day minimum \\
\hline Statistic & Discharge-- maximum a nnual 90 day \\
\hline Statistic & Discharge-- maximum a nnual 90 day \\
\hline Statistic & Discharge-- maximum a nnual 90 day \\
\hline Sta tis tic & Discharge-- maximum average April \\
\hline Statistic & Discharge-- maximum a verage August \\
\hline Statistic & Discharge-- maximum average December \\
\hline Statistic & Discharge-- maximum a verage Februa ry \\
\hline Statjstic & Discha rge-- maximum a verage Ja nua ry \\
\hline Statistic & Discharge-- maximum a verage July \\
\hline
\end{tabular}




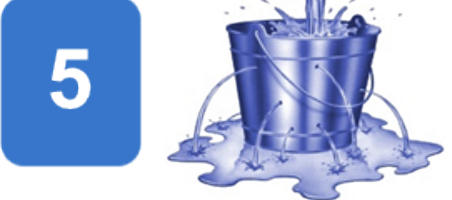

W1 Water Quantity

\begin{tabular}{|c|c|}
\hline Metric Type & Metric Name \\
\hline Statistic & Discharge-- maximum average June \\
\hline Statistic & Discharge--maximum average March \\
\hline Statistic & Discharge-- maximum average May \\
\hline Statistic & Discharge--maximum average November \\
\hline Statistic & Discharge--maximum average October \\
\hline Statistic & Discharge-- maximum average September \\
\hline Statistic & Discharge- maximum bas eflow index \\
\hline Statistic & Discharge-- maximum daily a verage outflow \\
\hline Statistic & Discharge-- maximum daily during \\
\hline Statistic & Discharge-- maximum daily first year \\
\hline Statistic & Discharge-- maximum daily flow previous \\
\hline Statistic & Discharge-- maximum duration high-flow \\
\hline Statistic & Discharge- maximum duration low-flow \\
\hline Statistic & Discharge--maximum fall rate \\
\hline Statistic & Discharge-- maximum frequency high-flow \\
\hline Statistic & Discharge- maximum frequency low-flow \\
\hline Statistic & Discharge- maximum generating \\
\hline Statistic & Discharge--maximum instantaneous \\
\hline Statistic & Discharge-- maximum Julian date annual \\
\hline Statistic & Discharge-- maximum Julian date annual \\
\hline Statistic & Discharge-- maximum number of zero-flow \\
\hline Statistic & Discharge- maximum rise rate \\
\hline Statistic & Discharge--maximum/ minimum flow ratio \\
\hline Statistic & Discharge-- maxumum \\
\hline Statistic & Discharge-- median daily \\
\hline Statistic & Discharge-- minimum 10 day during rearing \\
\hline Statistic & Discharge-- minimum 10 day during \\
\hline Statistic & Discharge-- minimum annual \\
\hline Statistic & Discharge-- minimum annual 1 day \\
\hline Statistic & Discharge-- minimum annual 1 day maximum \\
\hline Statistic & Discharge--minimum annual 1 day minimum \\
\hline
\end{tabular}

W1_Downstream discharge and hydrology
Measures that describe the magnitude, frequency, duration, periodicity, and timing of flows downstream of a hydropower facility, including changes to these characteristics

\begin{tabular}{|c|c|c|c|}
\hline Metric Type & Metric Name & Metric Type & Metric Na me \\
\hline Statistic & Discharge-- minimum a nnual 3 day minimum & Statistic & Discharge--minimum rise rate \\
\hline Statistic & Discharge-- minimum a nnual 30 day & Statistic & Discharge--moderate flood \\
\hline Statistic & Discharge-- minimum a nnual 30 day & Statistic & Discharge-- monthly \\
\hline Statistic & Discharge-- minimum a nnual 30 day & Statistic & Discharge-- monthly baseflow duration \\
\hline Statistic & Discharge-- minimum a nnual 7 day & Statistic & Discharge--monthly baseflow magnitude \\
\hline Statistic & Discharge-- minimum a nnual 7 day maximum & Statistic & Discharge-- normal flood \\
\hline Statistic & Discharge-- minimum a nnual 7 day minimum & \multirow[t]{2}{*}{ Statistic } & \multirow{2}{*}{$\begin{array}{l}\text { Discharge- number days within } 1 \text { standard } \\
\text { deviation of maximum }\end{array}$} \\
\hline Statistic & Discharge-- minimum a nnual 90 day & & \\
\hline Statistic & Discharge-- minimum a nnual 90 day & Statistic & Discharge--percent days flow equalled or \\
\hline Statistic & Discharge-- minimum a nnual 90 day & \multirow[t]{2}{*}{ Sta tistic } & \multirow{2}{*}{$\begin{array}{l}\text { Discharge- percent exceedance flow } \\
\text { threshold values average monthly discharge }\end{array}$} \\
\hline Statistic & Discharge-- minimum a nnual flow previous & & \\
\hline Statistic & Discharge-- minimum a verage April & \multirow[t]{2}{*}{ Sta tistic } & \multirow{2}{*}{$\begin{array}{l}\text { Discharge-- percent exceedance flow } \\
\text { threshold values maximum } 1 \text { day discharge }\end{array}$} \\
\hline Statistic & Discharge-- minimum a verage August & & \\
\hline Statistic & Discharge-- minimum average December & \multirow[t]{2}{*}{ Statistic } & \multirow{2}{*}{$\begin{array}{l}\text { Discharge- percent exceedance flow } \\
\text { threshold values maximum } 3 \text { day discharge }\end{array}$} \\
\hline Statistic & Discha rge-- minimum a verage February & & \\
\hline Statistic & Discharge-- minimum a verage Ja nuary & \multirow[t]{2}{*}{ Sta tistic } & \multirow{2}{*}{$\begin{array}{l}\text { Discharge- percent exceedance flow } \\
\text { threshold values maximum } 30 \text { day discharge }\end{array}$} \\
\hline Statistic & Discharge-- minimum a verage July & & \\
\hline Statistic & Discharge-- minimum average June & \multirow[t]{2}{*}{ Statistic } & \multirow{2}{*}{$\begin{array}{l}\text { Discharge- percent exceedance flow } \\
\text { threshold values maximum } 7 \text { day discharge }\end{array}$} \\
\hline Statistic & Discharge-- minimum a verage March & & \\
\hline Statistic & Discharge-- minimum a verage May & \multirow[t]{2}{*}{ Statistic } & \multirow{2}{*}{$\begin{array}{l}\text { Discharge- percent exceedance flow } \\
\text { threshold values maximum } 90 \text { day discharge }\end{array}$} \\
\hline Statistic & Discharge-- minimum a verage November & & \\
\hline Statistic & Discharge-- minimum a verage October & \multirow[t]{2}{*}{ Statistic } & \multirow{2}{*}{$\begin{array}{l}\text { Discharge- percent exceedance flow } \\
\text { threshold values minimum } 1 \text { day discharge }\end{array}$} \\
\hline Statistic & Discharge-- minimum a verage September & & \\
\hline Statistic & Discharge-- minimum baseflow index & \multirow[t]{2}{*}{ Sta tistic } & \multirow{2}{*}{$\begin{array}{l}\text { Discharge- percent exceedance flow } \\
\text { threshold values minimum } 3 \text { day discharge }\end{array}$} \\
\hline Statistic & Discharge-- minimum daily during incubation & & \\
\hline Statistic & Discharge-- minimum daily first year & \multirow[t]{2}{*}{ Statistic } & \multirow{2}{*}{$\begin{array}{l}\text { Discharge- percent exceedance flow } \\
\text { threshold values minimum } 30 \text { day discharge }\end{array}$} \\
\hline Statistic & Discharge-- minimum duration high-flow & & \\
\hline Statistic & Discharge-- minimum duration low-flow & \multirow[t]{2}{*}{ Sta tistic } & \multirow{2}{*}{$\begin{array}{l}\text { Discharge- percent exceedance flow } \\
\text { threshold values minimum } 7 \text { day discharge }\end{array}$} \\
\hline Statistic & Discharge-- minimum fall rate & & \\
\hline Statistic & Discharge-- minimum frequency high-flow & \multirow[t]{2}{*}{ Sta tistic } & \multirow{2}{*}{$\begin{array}{l}\text { Discharge- percent exceedance flow } \\
\text { threshold values minimum } 90 \text { day discharge }\end{array}$} \\
\hline Statistic & Discharge-- minimum frequency low-flow & & \\
\hline Statistic & Discharge-- minimum instantaneous & Statistic & Discharge-- percent exceedance flows \\
\hline Statistic & Discharge-- minimum Julian date annual & Statistic & Discharge-- Q7-10 flow \\
\hline Statistic & Discharge-- minimum Julian date annual & Statistic & Discharge--range daily \\
\hline Stetistatal & 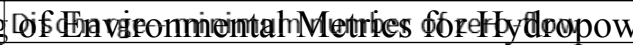 & & Discharge--ratio average month \\
\hline
\end{tabular}




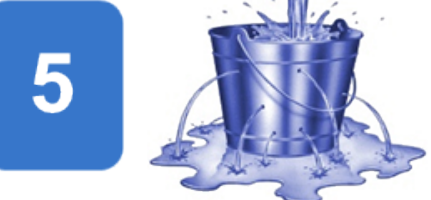

W1 Water Quantity

\begin{tabular}{|c|c|}
\hline Metric Type & Metric Na me \\
\hline Statistic & Discharge- rise rate \\
\hline Statistic & Discharge--severe flood \\
\hline Statistic & $\begin{array}{l}\text { Discharge-- standard deviation average } \\
\text { discharge during rearing period }\end{array}$ \\
\hline Statistic & $\begin{array}{l}\text { Discharge-- standard deviation average } \\
\text { discharge during spawning period }\end{array}$ \\
\hline Statistic & $\begin{array}{l}\text { Discharge-- standard deviation minimum } 10 \\
\text { d discharge during rearing period }\end{array}$ \\
\hline Statistic & $\begin{array}{l}\text { Discharge-- standard deviation minimum } 10 \\
\text { d discharge during spawning period }\end{array}$ \\
\hline Statistic & Discharge-- subdaily flow fluctuation \\
\hline Statistic & Discharge-- timinimumg high flow events \\
\hline Statistic & Discharge- total daily fres hwater inflow into \\
\hline Statistic & $\begin{array}{l}\text { Discharge-- total monthly freshwater inflow } \\
\text { into estuary }\end{array}$ \\
\hline Statistic & Discharge--va riability annual 1 day \\
\hline Statistic & Discharge--variability annual 1 day \\
\hline Statistic & Discharge--va riability annual 3 day \\
\hline Statistic & Discharge- va riability annual 3 day \\
\hline Statistic & Discharge- va riability annual 30 day \\
\hline Statistic & Discharge-- va riability annual 30 day \\
\hline Statistic & Discharge- va riability annual 7 day \\
\hline Statistic & Discharge- va riability annual 7 day \\
\hline Statistic & Discharge- va riability annual 90 day \\
\hline Statistic & Discharge- va riability annual 90 day \\
\hline Statistic & Discharge- va riability average April \\
\hline Statistic & Discharge- va riability average August \\
\hline Statistic & Discharge- va riability average December \\
\hline Statistic & Discharge- va riability average February \\
\hline Statistic & Discharge--va riability average Ja nua ry \\
\hline Statistic & Discharge-- va riability average July \\
\hline Statistic & Discharge--va riability average June \\
\hline Statistic & Discharge-- va riability average March \\
\hline
\end{tabular}

W1_Downstream discharge and hydrology
Measures that describe the magnitude, frequency, duration, periodicity, and timing of flows downstream of a hydropower facility, including changes to these characteristics

\begin{tabular}{|c|c|}
\hline Metric Type & Metric Name \\
\hline Statistic & Discharge-- va riability average May \\
\hline Statistic & Discharge- va riability average November \\
\hline Statistic & Discharge- va riability average October \\
\hline Statistic & Discharge- va riability average September \\
\hline Statistic & Discharge- va riability bas eflow index \\
\hline Statistic & Discharge--variability duration high-flow \\
\hline Statistic & Discharge- va riability duration low-flow \\
\hline Statistic & Discharge- va riability fall rate \\
\hline Statistic & Discharge--variability frequency high-flow \\
\hline Statistic & Discharge--variability frequency low-flow \\
\hline Statistic & Discharge--variability high-flow frequency \\
\hline Statistic & Discharge--variability Julian date annual \\
\hline Statistic & Discharge-- va riability Julian date annual \\
\hline Statistic & Discharge--va riability number of zero-flow \\
\hline Statistic & Discharge- va riability rise rate \\
\hline Statistic & Discharge wet year-- average monthly \\
\hline Statistic & Discharge-frequency zero-flow days \\
\hline Statistic & Down-ra mp rate-- average \\
\hline Statistic & Duration maximum variation annual \\
\hline Statistic & Exceptional flood discharge \\
\hline Statistic & Flow predictability \\
\hline Statistic & Ramping rate \\
\hline Statistic & Rate of channel dewatering \\
\hline Statistic & Rate of channel dewatering - maximum \\
\hline Statistic & Rate of channel dewatering - median \\
\hline Statistic & Ratio of flow constancy to flow predictability \\
\hline Statistic & Runoff \\
\hline Statistic & Spill event frequency \\
\hline Statistic & Spill event magnitude-- maximum \\
\hline Statistic & Spill water volume-- average daily \\
\hline Statistic & Tailwa ter el evation decrease \\
\hline Statistic & $\begin{array}{l}\text { Timing a nnual extremes Indicators of } \\
\text { Hydrologic Alteration variables-- coefficient }\end{array}$ \\
\hline Statistic & Total annual flow \\
\hline
\end{tabular}

\begin{tabular}{|l|l|}
\hline Metric Type & \multicolumn{1}{|c|}{ Metric Name } \\
\hline Statistic & Years between peak flows \\
\hline Indicator & Degree of regulation \\
\hline Indicator & Deviation baseflow index \\
\hline Indicator & Deviation frequency low-flow \\
\hline Indicator & Deviation high-flow frequency \\
\hline Indicator & $\begin{array}{l}\text { Discharge-- coefficient of variation percent } \\
\text { change pre-/ post-dam annual baseflow }\end{array}$ \\
\hline Indicator & Discharge-- deviation annual 1 day \\
\hline Indicator & Discharge-- deviation annual 1 day \\
\hline Indicator & Discharge-- deviation annual 1 day minimum \\
\hline Indicator & Discharge-- deviation annual 3 day \\
\hline Indicator & Discharge-- deviation annual 3 day minimum \\
\hline Indicator & Discharge-- deviation annual 3 day minimum \\
\hline Indicator & Discharge-- deviation annual 30 day \\
\hline Indicator & Discharge-- deviation annual 30 day \\
\hline Indicator & Discharge-- deviation annual 7 day \\
\hline Indicator & Discharge-- deviation annual 7 day minimum \\
\hline Indicator & Discharge-- deviation annual 90 day \\
\hline Indicator & Discharge-- deviation annual 90 day \\
\hline Indicator & Discharge-- deviation average April \\
\hline Indicator & Discharge-- deviation average August \\
\hline Indicator & Discharge-- deviation average December \\
\hline Indicator & Discharge-- deviation average February \\
\hline Indicator & Discharge-- deviation average January \\
\hline Indicator & Discharge-- deviation average July \\
\hline Indicator & Discharge-- deviation average June \\
\hline Indicator & Discharge-- deviation average March \\
\hline Indicator & Discharge-- deviation average May \\
\hline Indicator & Discharge-- deviation average November \\
\hline Indicator & Discharge-- deviation average October \\
\hline Indicator & Discharge-- deviation average September \\
\hline Indicator & Discharge-- deviation ba seflow index \\
\hline Indicator & Discharge-- deviation duration high-flow \\
\hline Indicator & Discharge-- deviation duration Iow-flow \\
\hline Indfizator & Discharge-- deviation fall rate \\
\hline
\end{tabular}




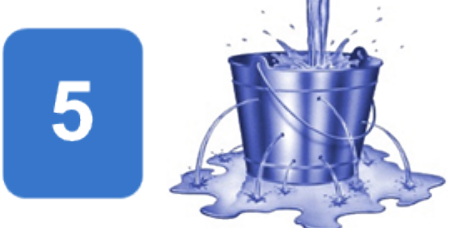

W1 Water Quantity

\begin{tabular}{|c|c|}
\hline Metric Type & Metric Name \\
\hline Indicator & Discharge-- deviation frequency low-flow \\
\hline Indicator & Discharge-- deviation high-flow frequency \\
\hline Indicator & Discharge-- deviation Julian date annual \\
\hline Indicator & Discharge-- deviation Julian date annual \\
\hline Indicator & Discharge-- deviation number of zero-flow \\
\hline Indicator & Discharge-- deviation rise rate \\
\hline Indicator & Discharge-- deviation annual 1 day maximum \\
\hline Indicator & Discharge-- deviation annual 1 day minimum \\
\hline Indicator & Discharge-- deviation annual 3 day maximum \\
\hline Indicator & Discharge-- deviation annual 3 day minimum \\
\hline Indicator & Discharge-- deviation annual 30 day \\
\hline Indicator & Discharge-- deviation annual 30 day \\
\hline Indicator & Discharge-- deviation annual 7 day maximum \\
\hline Indicator & Discharge-- deviation annual 7 day minimum \\
\hline Indicator & Discharge-- deviation annual 90 day \\
\hline Indicator & Discharge-- deviation annual 90 day \\
\hline Indicator & Discharge-- deviation average April \\
\hline Indicator & Discharge-- deviation average August \\
\hline Indicator & Discharge-- deviation average December \\
\hline Indicator & Discharge-- deviation average February \\
\hline Indicator & Discharge-- deviation average January \\
\hline Indicator & Discharge-- deviation avera ge July \\
\hline Indicator & Discharge-- deviation average June \\
\hline Indicator & Discharge-- deviation average March \\
\hline Indicator & Discharge-- deviation average May \\
\hline Indicator & Discharge-- deviation average November \\
\hline Indicator & Discharge-- deviation average October \\
\hline Indicator & Discharge-- deviation average September \\
\hline Indicator & Discharge-- deviation baseflow index \\
\hline Indicator & Discharge-- deviation duration high-flow \\
\hline Indicator & Discharge-- deviation duration Iow-flow \\
\hline
\end{tabular}

W1_Downstream discharge and hydrology
Measures that describe the magnitude, frequency, duration, periodicity, and timing of flows downstream of a hydropower facility,

\section{including changes to these characteristics}

\begin{tabular}{|c|c|}
\hline Metric Type & $\begin{array}{r}\text { Metric Na me } \\
\end{array}$ \\
\hline Indicator & Discharge-- deviation fall rate \\
\hline Indicator & Discharge-- deviation frequency high-flow \\
\hline Indicator & Discharge-- deviation frequency low-flow \\
\hline Indicator & Discharge-- deviation in high flow event \\
\hline Indicator & Discharge-- deviation Julian date annual \\
\hline Indicator & Discharge-- deviation Julian date annual \\
\hline Indicator & Discharge-- deviation number of zero-flow \\
\hline Indicator & Discharge-- deviation rise rate \\
\hline Indicator & $\begin{array}{l}\text { Discharge-- number days discharge exceeds } \\
\text { thresholds }\end{array}$ \\
\hline Indicator & Discharge-- percent change annual 1 day \\
\hline Indicator & Discharge-- percent change annual 1 day \\
\hline Indicator & Discharge-- percent change annual 3 day \\
\hline Indicator & Discharge-- percent change annual 3 day \\
\hline Indicator & Discharge-- percent change annual 30 day \\
\hline Indicator & Discharge-- percent change annual 30 day \\
\hline Indicator & Discharge-- percent change annual 7 day \\
\hline Indicator & Discharge-- percent change annual 7 day \\
\hline Indicator & Discharge-- percent change annual 90 day \\
\hline Indicator & Discharge-- percent change annual 90 day \\
\hline Indicator & Discharge-- percent change average April \\
\hline Indicator & Discharge-- percent change average August \\
\hline Indicator & Discharge-- percent change average \\
\hline Indicator & Discharge-- percent change average February \\
\hline Indicator & Discharge-- percent change average January \\
\hline Indicator & Discharge-- percent change average July \\
\hline Indicator & Discharge-- percent change average June \\
\hline Indicator & Discharge-- percent change average March \\
\hline Indicator & Discharge-- percent change average May \\
\hline Indicator & Discharge-- percent change average \\
\hline Indicator & Discharge-- percent change average October \\
\hline Indicator & Discharge-- percent change average \\
\hline Indicator & Discharge-- percent change baseflow index \\
\hline Indicator & Discharge-- percent change duration high- \\
\hline Indicator & Discharge-- percent change duration high- \\
\hline
\end{tabular}

\begin{tabular}{|c|c|}
\hline Metric Type & Metric Name \\
\hline Indicator & Discharge-- percent change duration low-flow \\
\hline Indicator & Discharge-- percent change fall rate \\
\hline Indicator & Discharge-- percent change frequency high- \\
\hline Indicator & Discharge-- percent change frequency low- \\
\hline Indicator & Discharge-- percent change high-flow \\
\hline Indicator & $\begin{array}{l}\text { Discharge-- percent change Julian date } \\
\text { a nnual maximum }\end{array}$ \\
\hline Indicator & $\begin{array}{l}\text { Discharge-- percent change Julian date } \\
\text { a nnual minimum }\end{array}$ \\
\hline Indicator & Discharge-- percent change number of zero- \\
\hline Indicator & $\begin{array}{l}\text { Discharge-- percent change pre-/ post-dam } \\
\text { a nnual baseflow }\end{array}$ \\
\hline Indicator & $\begin{array}{l}\text { Discharge-- percent change pre-/ post-dam } \\
\text { a nnual predictability }\end{array}$ \\
\hline Indicator & $\begin{array}{l}\text { Discharge-- percent change pre-/ post-dam } \\
\text { count high-flow pulse }\end{array}$ \\
\hline Indicator & $\begin{array}{l}\text { Discharge-- percent change pre-/ post-dam } \\
\text { duration high-flow pulse }\end{array}$ \\
\hline Indicator & $\begin{array}{l}\text { Discharge-- percent change pre-/ post-dam } \\
\text { ratio of flow constancy to flow predictability }\end{array}$ \\
\hline Indicator & Discharge-- percent change rise rate \\
\hline Indicator & $\begin{array}{l}\text { Discharge-- percent months environmental } \\
\text { flow requirement met }\end{array}$ \\
\hline Indicator & Discharge-- rva target high annual 1 day \\
\hline Indicator & Discharge-- rva target high annual 1 day \\
\hline Indicator & Discharge-- rva target high annual 3 day \\
\hline Indicator & Discharge-- rva target high annual 3 day \\
\hline Indicator & Discharge-- rva target high annual 30 day \\
\hline Indicator & Discharge-- rva target high annual 30 day \\
\hline Indicator & Discharge-- rva target high annual 7 day \\
\hline Indicator & Discharge-- rva target high annual 7 day \\
\hline Indicator & Discharge-- rva target high annual 90 day \\
\hline Indicator & Discharge--rva target high annual 90 day \\
\hline Indicator & Discharge-- rva target high average April \\
\hline & Discharge-- rva target high average Augu \\
\hline
\end{tabular}




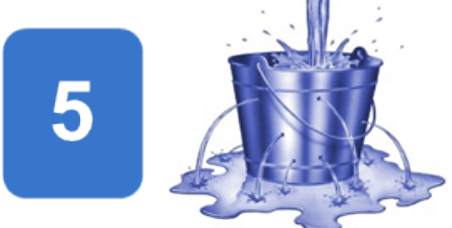

W1 Water Quantity

\begin{tabular}{|c|c|}
\hline Metric Type & Metric Name \\
\hline Indicator & Discharge-- deviation frequency low-flow \\
\hline Indicator & Discharge-- deviation high-flow frequency \\
\hline Indicator & Discharge-- deviation Julian date annual \\
\hline Indicator & Discharge-- deviation Julian date annual \\
\hline Indicator & Discharge-- deviation number of zero-flow \\
\hline Indicator & Discharge-- deviation rise rate \\
\hline Indicator & Discharge-- deviation annual 1 day maximum \\
\hline Indicator & Discharge-- deviation annual 1 day minimum \\
\hline Indicator & Discharge-- deviation annual 3 day maximum \\
\hline Indicator & Discharge-- deviation annual 3 day minimum \\
\hline Indicator & Discharge-- deviation annual 30 day \\
\hline Indicator & Discharge-- deviation annual 30 day \\
\hline Indicator & Discharge-- deviation annual 7 day maximum \\
\hline Indicator & Discharge-- deviation annual 7 day minimum \\
\hline Indicator & Discharge-- deviation annual 90 day \\
\hline Indicator & Discharge-- deviation annual 90 day \\
\hline Indicator & Discharge-- deviation average April \\
\hline Indicator & Discharge-- deviation average August \\
\hline Indicator & Discharge-- deviation average December \\
\hline Indicator & Discharge-- deviation average February \\
\hline Indicator & Discharge-- deviation average January \\
\hline Indicator & Discharge-- deviation avera ge July \\
\hline Indicator & Discharge-- deviation average June \\
\hline Indicator & Discharge-- deviation average March \\
\hline Indicator & Discharge-- deviation average May \\
\hline Indicator & Discharge-- deviation average November \\
\hline Indicator & Discharge-- deviation average October \\
\hline Indicator & Discharge-- deviation average September \\
\hline Indicator & Discharge-- deviation baseflow index \\
\hline Indicator & Discharge-- deviation duration high-flow \\
\hline Indicator & Discharge-- deviation duration Iow-flow \\
\hline
\end{tabular}

W1_Downstream discharge and hydrology
Measures that describe the magnitude, frequency, duration, periodicity, and timing of flows downstream of a hydropower facility,

\section{including changes to these characteristics}

\begin{tabular}{|c|c|}
\hline Metric Type & $\begin{array}{r}\text { Metric Na me } \\
\end{array}$ \\
\hline Indicator & Discharge-- deviation fall rate \\
\hline Indicator & Discharge-- deviation frequency high-flow \\
\hline Indicator & Discharge-- deviation frequency low-flow \\
\hline Indicator & Discharge-- deviation in high flow event \\
\hline Indicator & Discharge-- deviation Julian date annual \\
\hline Indicator & Discharge-- deviation Julian date annual \\
\hline Indicator & Discharge-- deviation number of zero-flow \\
\hline Indicator & Discharge-- deviation rise rate \\
\hline Indicator & $\begin{array}{l}\text { Discharge-- number days discharge exceeds } \\
\text { thresholds }\end{array}$ \\
\hline Indicator & Discharge-- percent change annual 1 day \\
\hline Indicator & Discharge-- percent change annual 1 day \\
\hline Indicator & Discharge-- percent change annual 3 day \\
\hline Indicator & Discharge-- percent change annual 3 day \\
\hline Indicator & Discharge-- percent change annual 30 day \\
\hline Indicator & Discharge-- percent change annual 30 day \\
\hline Indicator & Discharge-- percent change annual 7 day \\
\hline Indicator & Discharge-- percent change annual 7 day \\
\hline Indicator & Discharge-- percent change annual 90 day \\
\hline Indicator & Discharge-- percent change annual 90 day \\
\hline Indicator & Discharge-- percent change average April \\
\hline Indicator & Discharge-- percent change average August \\
\hline Indicator & Discharge-- percent change average \\
\hline Indicator & Discharge-- percent change average February \\
\hline Indicator & Discharge-- percent change average January \\
\hline Indicator & Discharge-- percent change average July \\
\hline Indicator & Discharge-- percent change average June \\
\hline Indicator & Discharge-- percent change average March \\
\hline Indicator & Discharge-- percent change average May \\
\hline Indicator & Discharge-- percent change average \\
\hline Indicator & Discharge-- percent change average October \\
\hline Indicator & Discharge-- percent change average \\
\hline Indicator & Discharge-- percent change baseflow index \\
\hline Indicator & Discharge-- percent change duration high- \\
\hline Indicator & Discharge-- percent change duration high- \\
\hline
\end{tabular}

\begin{tabular}{|c|c|}
\hline Metric Type & Metric Name \\
\hline Indicator & Discharge-- percent change duration low-flow \\
\hline Indicator & Discharge-- percent change fall rate \\
\hline Indicator & Discharge-- percent change frequency high- \\
\hline Indicator & Discharge-- percent change frequency low- \\
\hline Indicator & Discharge-- percent change high-flow \\
\hline Indicator & $\begin{array}{l}\text { Discharge-- percent change Julian date } \\
\text { a nnual maximum }\end{array}$ \\
\hline Indicator & $\begin{array}{l}\text { Discharge-- percent change Julian date } \\
\text { a nnual minimum }\end{array}$ \\
\hline Indicator & Discharge-- percent change number of zero- \\
\hline Indicator & $\begin{array}{l}\text { Discharge-- percent change pre-/ post-dam } \\
\text { a nnual baseflow }\end{array}$ \\
\hline Indicator & $\begin{array}{l}\text { Discharge-- percent change pre-/ post-dam } \\
\text { a nnual predictability }\end{array}$ \\
\hline Indicator & $\begin{array}{l}\text { Discharge-- percent change pre-/ post-dam } \\
\text { count high-flow pulse }\end{array}$ \\
\hline Indicator & $\begin{array}{l}\text { Discharge-- percent change pre-/ post-dam } \\
\text { duration high-flow pulse }\end{array}$ \\
\hline Indicator & $\begin{array}{l}\text { Discharge-- percent change pre-/ post-dam } \\
\text { ratio of flow constancy to flow predictability }\end{array}$ \\
\hline Indicator & Discharge-- percent change rise rate \\
\hline Indicator & $\begin{array}{l}\text { Discharge-- percent months environmental } \\
\text { flow requirement met }\end{array}$ \\
\hline Indicator & Discharge-- rva target high annual 1 day \\
\hline Indicator & Discharge-- rva target high annual 1 day \\
\hline Indicator & Discharge-- rva target high annual 3 day \\
\hline Indicator & Discharge-- rva target high annual 3 day \\
\hline Indicator & Discharge-- rva target high annual 30 day \\
\hline Indicator & Discharge-- rva target high annual 30 day \\
\hline Indicator & Discharge-- rva target high annual 7 day \\
\hline Indicator & Discharge-- rva target high annual 7 day \\
\hline Indicator & Discharge-- rva target high annual 90 day \\
\hline Indicator & Discharge--rva target high annual 90 day \\
\hline Indicator & Discharge-- rva target high average April \\
\hline & Discharge-- rva target high average Augu \\
\hline
\end{tabular}


W1_Downstream

discharge and hydrology
Measures that describe the magnitude, frequency, duration, periodicity, and timing of flows downstream of a hydropower facility, including changes to these characteristics

\section{W1 Water Quantity}

\begin{tabular}{|c|c|}
\hline Metric Type & Metric Name \\
\hline Indicator & Discharge-- rva target high average December \\
\hline Indicator & Discharge-- rva target high average February \\
\hline Indicator & Discharge-- rva target high average January \\
\hline Indicator & Discharge-- rva target high average July \\
\hline Indicator & Discharge-- rva target high average June \\
\hline Indicator & Discharge-- rva target high average March \\
\hline Indicator & Discharge-- rva target high average May \\
\hline Indicator & Discharge-- rva target high average November \\
\hline Indicator & Discharge-- rva target high average October \\
\hline Indicator & Discharge-- rva target high average \\
\hline Indicator & Discharge-- rva target high baseflow index \\
\hline Indicator & Discharge-- rva target high duration high- \\
\hline Indicator & Discharge-- rva target high duration low-flow \\
\hline Indicator & Discharge-- rva target high fall rate \\
\hline Indicator & Discharge-- rva target high frequency high- \\
\hline Indicator & Discharge-- rva target high frequency low- \\
\hline Indicator & $\begin{array}{l}\text { Discharge-- rva target high Julian date annual } \\
\text { maximum }\end{array}$ \\
\hline Indicator & $\begin{array}{l}\text { Discharge-- rva target high Julian date annual } \\
\text { minimum }\end{array}$ \\
\hline Indicator & Discharge-- rva target high number of zero- \\
\hline Indicator & Discharge-- rva target high rise rate \\
\hline Indicator & Discharge-- rva target low annual 1 day \\
\hline Indicator & Discharge-- rva target low annual 1 day \\
\hline Indicator & Discharge-- rva target low annual 3 day \\
\hline Indicator & Discharge-- rva target low annual 3 day \\
\hline Indicator & Discharge-- rva target low annual 30 day \\
\hline Indicator & Discharge-- rva target low annual 30 day \\
\hline Indicator & Discharge-- rva target low annual 7 day \\
\hline Indicator & Discharge-- rva target low annual 7 day \\
\hline Indicator & Discharge-- rva target low annual 90 day \\
\hline Indicator & Discharge-- rva target low annual 90 day \\
\hline Indicator & Discharge-- rva target low average April \\
\hline Indicator & Discharge- rva target low average Augus \\
\hline Indicator & \\
\hline
\end{tabular}

\begin{tabular}{|c|c|}
\hline Metric Type & Metric Na me \\
\hline Indicator & Discharge-- rva target low a verage February \\
\hline Indicator & Discharge- rva ta rget low a verage January \\
\hline Indicator & Discharge--rva ta rget low a verage July \\
\hline Indicator & Discharge- rva target low a verage June \\
\hline Indicator & Discharge--rva target low a verage March \\
\hline Indicator & Discharge--rva target low a verage May \\
\hline Indicator & Discharge- rva ta rget low a verage November \\
\hline Indicator & Discharge- rva ta rget low a verage October \\
\hline Indicator & Discharge-- rva ta rget low a verage September \\
\hline Indicator & Discharge- rva target low baseflow index \\
\hline Indicator & Discharge--rva target low duration high-flow \\
\hline Indicator & Discharge--rva target low duration low-flow \\
\hline Indicator & Discharge- rva ta rget low fall rate \\
\hline Indicator & Discharge-- rva ta rget low frequency high- \\
\hline Indicator & Discharge-- rva target low frequency low-flow \\
\hline Indicator & $\begin{array}{l}\text { Discharge- rva ta rget low Julian date annual } \\
\text { maximum }\end{array}$ \\
\hline Indicator & $\begin{array}{l}\text { Discharge- rva ta rget low Julian date annual } \\
\text { minimum }\end{array}$ \\
\hline Indicator & Discharge-- rva target low number of zero- \\
\hline Indicator & Discharge--rva target low rise rate \\
\hline Indicator & Dry season minimum flow \\
\hline Indicator & Dundee hydrological regime assessment \\
\hline Indicator & General release monitoring \\
\hline Indicator & Maximum distance between points \\
\hline Indicator & $\begin{array}{l}\text { Studies of risks of flow allocation and } \\
\text { variation on aquatic biota }\end{array}$ \\
\hline Indicator & Tailwater el evation fluctuation \\
\hline
\end{tabular}

Indicator Discharge--rva targe Iow average December 
3.5.4 W1_Groundwater

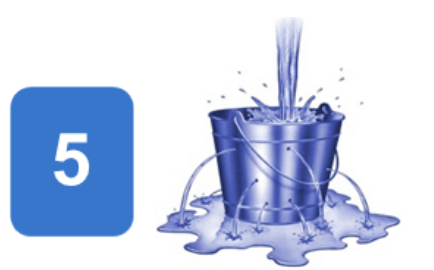

W1_Groundwater

Groundwater characteristics

W1 Water Quantity

\begin{tabular}{|l|r|r|}
\hline Sector & studies metrics \\
\hline IHA & 4 & 1 \\
\hline LIHI & 5 & 0 \\
\hline FERC & 5 & 0 \\
\hline Peer- & & \\
Reviewed & & \\
Literature & 247 & 21 \\
\hline \multicolumn{1}{|r|}{ Totals } & 261 & 22 \\
\hline
\end{tabular}

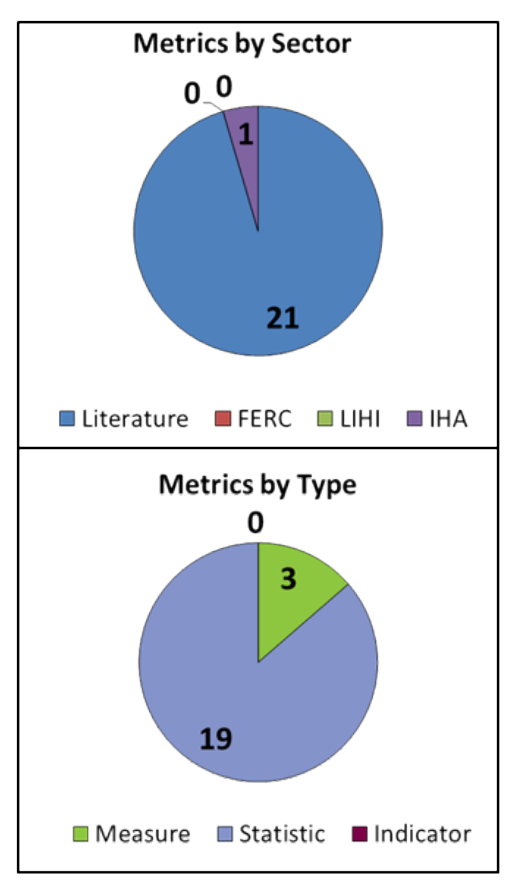

\begin{tabular}{|l|l|}
\hline Metric Type & \multicolumn{1}{|c|}{ Metric Name } \\
\hline Measure & Groundwater discharge \\
\hline Measure & Groundwater elevation \\
\hline Measure & Groundwater storage \\
\hline Statistic & Groundwater elevation-- average post-dam \\
\hline Statistic & Groundwater elevation-- average pre-dam dry year \\
\hline Statistic & Groundwater elevation-- average standard devation pre-dam dry year \\
\hline Statistic & Groundwater elevation-- average standard deviation post-dam \\
\hline Statistic & Groundwater elevation-- average standard deviation pre-dam dry year \\
\hline Statistic & Groundwater elevation-- skewness post-dam \\
\hline Statistic & Groundwater elevation-- skewness pre-dam \\
\hline Statistic & Groundwater elevation-- skewness pre-dam dry year \\
\hline Statistic & Groundwater elevation-- standard deviation of standard deviations post-dam \\
\hline Statistic & Groundwater elevation-- standard deviation of standard deviations pre-dam \\
\hline Statistic & Groundwater elevation-- standard deviation of standard deviations pre-dam dry year \\
\hline Statistic & Groundwater elevation-- standard deviation post-dam \\
\hline Statistic & Groundwater elevation-- standard deviation pre-dam \\
\hline Statistic & Groundwater elevation-- standard deviation pre-dam dry year \\
\hline Statistic & Groundwater runoff contributions \\
\hline Statistic & Grounwater elevation-- skewness of standard deviations post-dam \\
\hline Statistic & Grounwater elevation-- skewness of standard deviations pre-dam \\
\hline Statistic & Grounwater elevation-- skewness of standard deviations pre-dam dry year \\
\hline
\end{tabular}

ORNL Catalog of Environmental Metrics for Hydropower - 57 


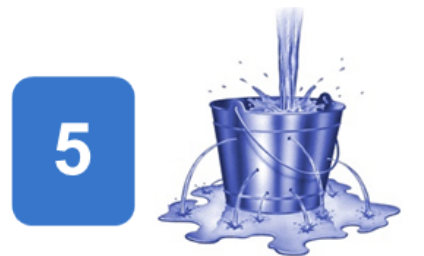

\section{W1 Water Quantity}

\begin{tabular}{|l|r|r|}
\hline Sector & studies metrics \\
\hline IHA & 4 & 3 \\
\hline LIHI & 5 & 18 \\
\hline FERC & 5 & 25 \\
\hline Peer- & & \\
Reviewed & & \\
Literature & 247 & 39 \\
\hline \multicolumn{1}{|r|}{ Totals } & 261 & 85 \\
\hline
\end{tabular}

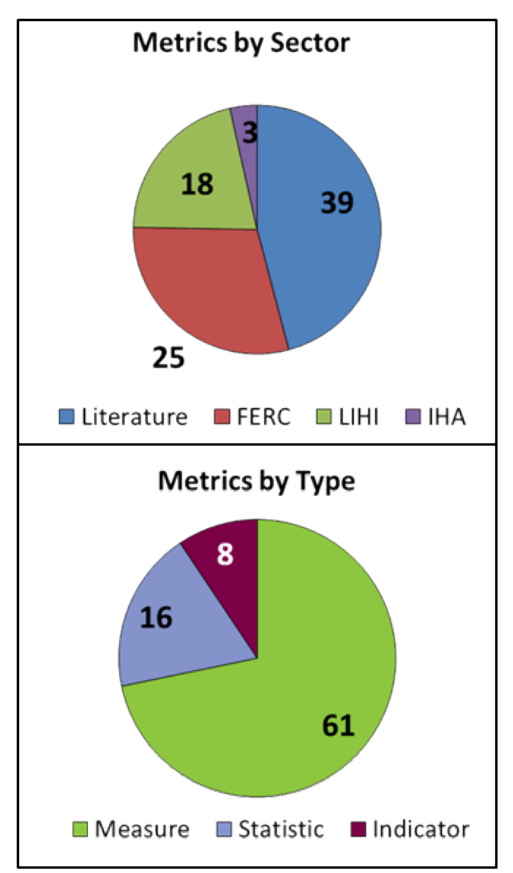

W1_Reservoir hydrology

Reservoir hydrological characteristics such as residence time, reservoir fluctuation, reservoir surface area, or degree of regulation

\begin{tabular}{|c|c|}
\hline Metric Type & Metric Name \\
\hline Me asure & Active reservoir storage \\
\hline Me asure & Dead reservoir storage \\
\hline Measure & Detailed water level measurements \\
\hline Me asure & Headwater elevations-- daily \\
\hline Measure & Hydraulic residence time \\
\hline Measure & Hydraulic residence time-- minimum \\
\hline Measure & Impounded water volume \\
\hline Me asure & Impoundment hydrology \\
\hline Me asure & Inundation volume-- average \\
\hline Measure & Number reservoir tributaries \\
\hline Me asure & Reservoir drawdown-- seasonal \\
\hline Measure & Reservoir elevation \\
\hline Measure & Reservoir elevation-- daily \\
\hline Measure & Reservoir elevation full pool \\
\hline Measure & Reservoir elevation-- hourly \\
\hline Me asure & Reservoirfill timing \\
\hline Measure & Reservoirinflow \\
\hline Me asure & $\begin{array}{l}\text { Reservoir precipitation minimum } \\
\text { evaporation }\end{array}$ \\
\hline Me asure & Reservoir storage \\
\hline Me asure & Reservoir storage-- maximum \\
\hline Measure & Reservoir surface area \\
\hline Me asure & Reservoir surface evaporation \\
\hline Me asure & Reservoir use class \\
\hline Me asure & Reservoir volume \\
\hline Me asure & Reservoir water depth \\
\hline Me asure & Stream water de pth \\
\hline Measure & Useable reservoir storage volume \\
\hline
\end{tabular}

\begin{tabular}{|l|l|}
\hline Metric Type & \multicolumn{1}{|c|}{ Metric Name } \\
\hline Measure & Water column dispersive transport \\
\hline Measure & Water depth \\
\hline Measure & Water depth reservoir \\
\hline Measure & Water depth reservoir-- maximum \\
\hline Statistic & $\begin{array}{l}\text { Discharge-- 25th percentile average } \\
\text { monthly flow }\end{array}$ \\
\hline Statistic & $\begin{array}{l}\text { Discharge-- percent useable reservoir } \\
\text { storage volume }\end{array}$ \\
\hline Statistic & Gauge height \\
\hline Statistic & Hydraulic residence time-- average \\
\hline Statistic & Hydraulic residence time-- maximum \\
\hline Statistic & $\begin{array}{l}\text { Net evaporation depth-- average } \\
\text { monthly }\end{array}$ \\
\hline Statistic & Normal daily reservoir drawdown \\
\hline Statistic & Reservoir drawdown-- maximum \\
\hline Statistic & $\begin{array}{l}\text { Reservoir elevation-- average daily } \\
\text { fluctuation }\end{array}$ \\
\hline Statistic & Reservoir elevation-- average hourly \\
\hline Statistic & $\begin{array}{l}\text { Reservoir elevation fluctuation-- } \\
\text { maximum }\end{array}$ \\
\hline Statistic & Water depth-- average \\
\hline Statistic & Water depth-- maximum \\
\hline Statistic & Water depth reservoir-- average \\
\hline Indicator & $\begin{array}{l}\text { Continuous monitoring of water levels } \\
\text { \& flow }\end{array}$ \\
\hline Indicator & Reservoir elevation fluctuation \\
\hline Indicator & Reservoir level \\
\hline
\end{tabular}

ORNL Catalog of Environmental Metrics for Hydropower - 58 


\subsubsection{W1_Upstream regulation and inflow}
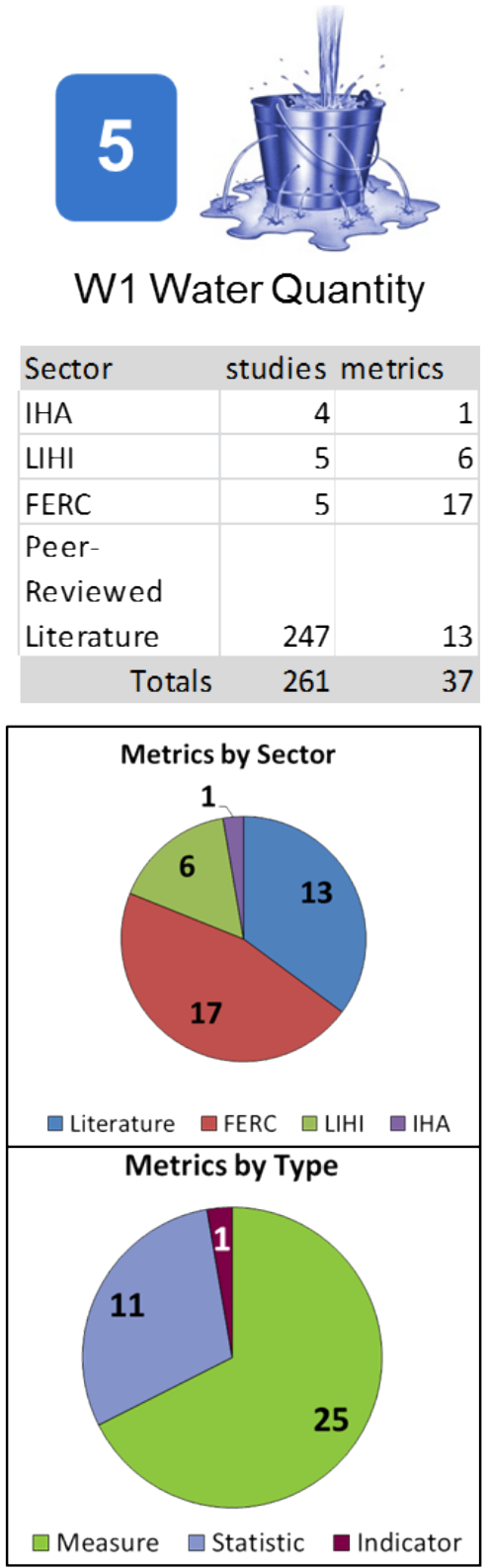

W1_Upstream

regulation and inflow
Measures describing the magnitude, frequency, duration, periodicity, and timing of flows upstream of a hydropower facility, including changes to these characteristics

\begin{tabular}{|l|l|}
\hline Metric Type & \multicolumn{1}{|c|}{ Metric Name } \\
\hline Measure & Count upstream dams \\
\hline Measure & Discharge-- average daily inflow \\
\hline Measure & Discharge-- inflow \\
\hline Measure & Discharge-- instantaneous inflow \\
\hline Measure & Discharge-- median inflow \\
\hline Measure & Discharge-- net inflow \\
\hline Measure & Discharge of small tributary inflows \\
\hline Measure & Discharge-- regulated inflow \\
\hline Measure & Discharge-- reservoir inflow \\
\hline Measure & Discharge-- river inflow \\
\hline Measure & Discharge-- unregulated inflow \\
\hline Measure & Discharge-- water balance estimated \\
\hline Measure & Distance upstream dams \\
\hline Measure & Number upstream dams \\
\hline Measure & Precipitation \\
\hline Measure & $\begin{array}{l}\text { Precipitation falling directly into the } \\
\text { reservoir }\end{array}$ \\
\hline Measure & Reservoir inflow \\
\hline Measure & Upstream area regulated \\
\hline Measure & Water use in region \\
\hline Statistic & Air temperature-- average July \\
\hline Statistic & $\begin{array}{l}\text { Air temperature-- difference average } \\
\text { January and July }\end{array}$ \\
\hline Statistic & Discharge-- average tributary inflow \\
\hline Statistic & $\begin{array}{l}\text { Discharge-- average unregulated } \\
\text { inflow }\end{array}$ \\
\hline Statistic & Discharge-- median daily inflow \\
\hline
\end{tabular}

\begin{tabular}{|l|l|}
\hline Metric Type & \multicolumn{1}{|c|}{ Metric Name } \\
\hline Statistic & $\begin{array}{l}\text { Discharge-- percent inflow to } \\
\text { mainstem }\end{array}$ \\
\hline Statistic & $\begin{array}{l}\text { Discharge-- percent of inflow from } \\
\text { upstream facility }\end{array}$ \\
\hline Statistic & $\begin{array}{l}\text { Discharge-- percent upstream area } \\
\text { regulated }\end{array}$ \\
\hline Statistic & Drainage area be tween facilities \\
\hline Statistic & $\begin{array}{l}\text { Precipitation-- ave rage annual in } \\
\text { catchment }\end{array}$ \\
\hline Statistic & Rainfall-- average annual \\
\hline Indicator & Flow regime of upper Isar \\
\hline
\end{tabular}

ORNL Catalog of Environmental Metrics for Hydropower - 59 
3.6 W2_Water Quality

6

Water quality characteristics such as the temperature and concentrations of dissolved oxygen, velocity, nutrients, and contaminants can be directly or indirectly affected by hydropower development and operation. Changes in water quality can adversely affect the health of humans and wildlife.

\section{W2 Water Quality}

\begin{tabular}{|c|c|c|}
\hline Sector & \# of studies & \# of metrics \\
\hline IHA & 4 & 38 \\
\hline LIHI & 5 & 8 \\
\hline FERC & 5 & 36 \\
\hline Peer-Reviewed & & \\
\hline Lite rature & 247 & 879 \\
\hline Totals & 261 & 961 \\
\hline
\end{tabular}

Water quality parameter frequency at study-level (extraction table) Primary

Category Parameter

Water WQ_Algae, primary productivity

WQ_Buffering capacity

WQ_Dissolved gasses

WQ_Dissolved oxygen

WQ_Ecosystem function

WQ_Gas emissions

WQ_Gas emmissions

WQ_Nutrients

WQ_Organic material

WQ_Other elements

WQ_Pollutants

WQ_Solid transport, turbidity, and conductivity

WQ_Temperature

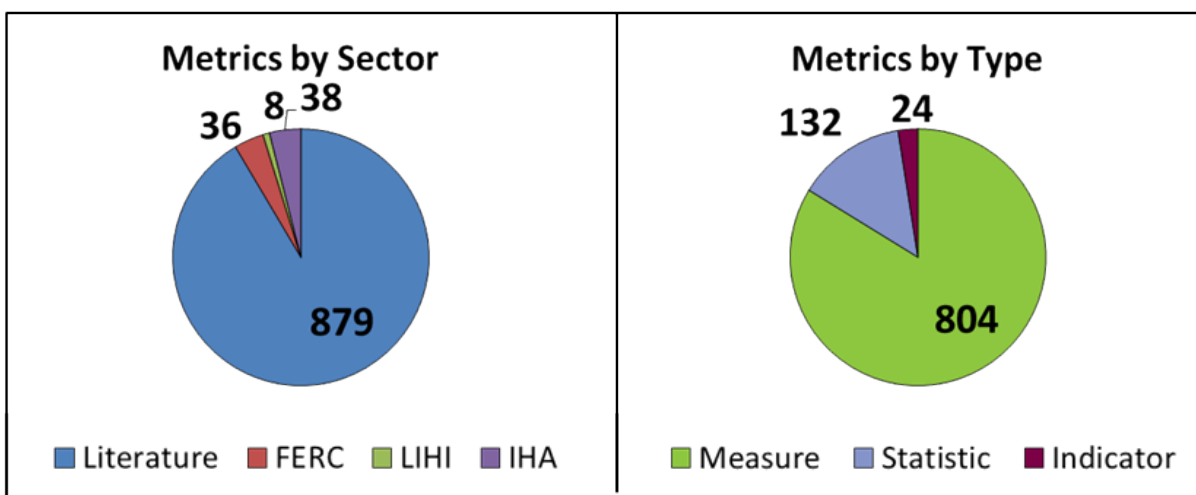

Spatial scales for water quality represented at study-level (extraction table)

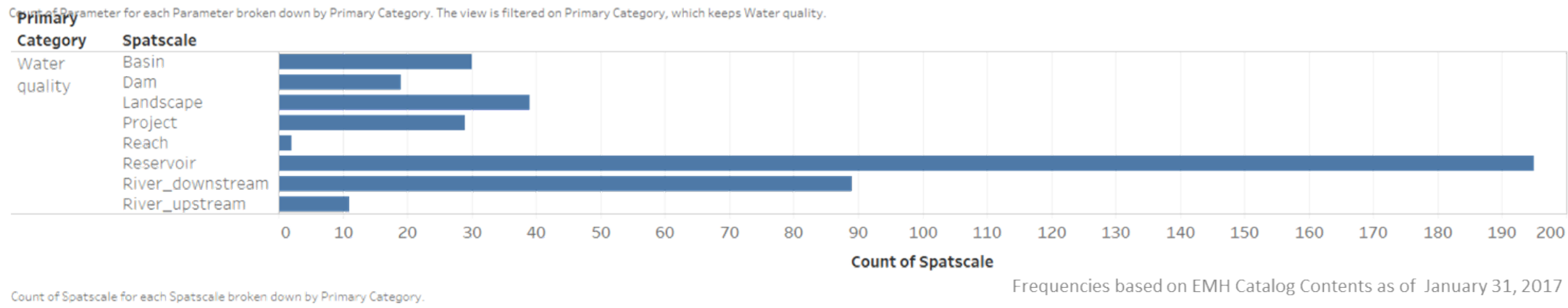

ORNL Catalog of Environmental Metrics for Hydropower - 60 
3.6.1 W2_Algae, Primary Productivity

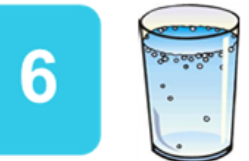

W2 Water Quality

\begin{tabular}{|l|r|r|}
\hline Sector & studies metrics \\
\hline IHA & 4 & 3 \\
\hline LIHI & 5 & 0 \\
\hline FERC & 5 & 0 \\
\hline Peer- & & \\
Reviewed & & \\
Literature & 247 & 31 \\
\hline \multicolumn{1}{|r|}{ Totals } & 261 & 34 \\
\hline
\end{tabular}

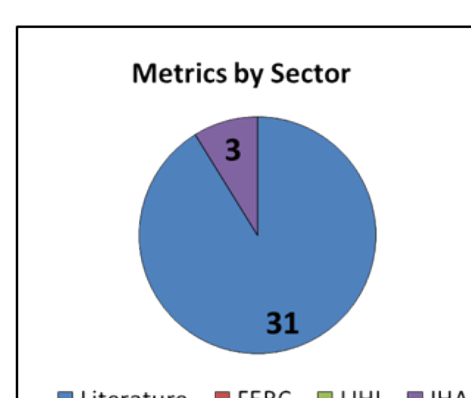

$\square$ Literature $\square$ FERC $\square$ LIHI $\square$ IHA

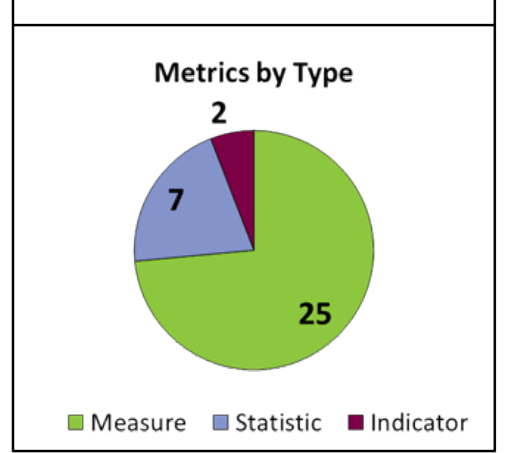

W2_Algae, primary productivity
Algal concentration, including measures of primary productivity such as chlorophyll $\mathrm{A}$ or cyanotoxin

\begin{tabular}{|l|l|}
\hline Metric Type & \multicolumn{1}{|c|}{ Metric Name } \\
\hline Measure & Benthic light availability \\
\hline Measure & Biochemical oxygen demand \\
\hline Measure & $\begin{array}{l}\text { Broad band photosynthetically active } \\
\text { radiation }\end{array}$ \\
\hline Measure & Chlorophyl a abundance \\
\hline Measure & Chlorophyll a \\
\hline Measure & Chlorophyll a abundance \\
\hline Measure & Downwelling irradiance \\
\hline Measure & $\begin{array}{l}\text { Downwelling irradiance; Attenuation } \\
\text { coefficient at depth z }\end{array}$ \\
\hline Measure & Eutrophication \\
\hline Measure & $\begin{array}{l}\text { Individual particle analysis in per area } \\
\text { volume }\end{array}$ \\
\hline Measure & Irradiance \\
\hline Measure & Irradiance cosine \\
\hline Measure & $\begin{array}{l}\text { Irradiance; Attenuation coefficient at } \\
\text { depth z }\end{array}$ \\
\hline Measure & Reflectance \\
\hline Measure & Scalar irradiance \\
\hline Measure & Seston \\
\hline Measure & Spectral downwelling irradiance \\
\hline Measure & Total chlorophyll \\
\hline Measure & Transimissometry \\
\hline Measure & Upwelling irradiance \\
\hline Measure & Water column food availability \\
\hline Statistic & Absorption coefficient \\
\hline Statistic & Modeled reservoir algae levels \\
\hline Statistic & Scattering coefficient \\
\hline
\end{tabular}

\begin{tabular}{|l|l|}
\hline Metric Type & \multicolumn{1}{|c|}{ Metric Name } \\
\hline Statistic & $\begin{array}{l}\text { Spectral average absorption } \\
\text { coefficient for gelbstoff }\end{array}$ \\
\hline Statistic & $\begin{array}{l}\text { Spectral average absorption } \\
\text { coefficient for particles }\end{array}$ \\
\hline Statistic & $\begin{array}{l}\text { Spectral average absorption } \\
\text { coefficient for water }\end{array}$ \\
\hline Statistic & Water depth euphotic zone \\
\hline Indicator & Monitoring of algal blooms \\
\hline Indicator & $\begin{array}{l}\text { Monitoring of algal proliferation } \\
\text { compared to baseline }\end{array}$ \\
\hline
\end{tabular}

ORNL Catalog of Environmental Metrics for Hydropower - 61 


\subsubsection{W2_Buffering Capacity}

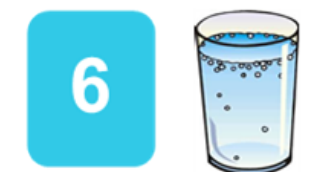

W2 Water Quality

\begin{tabular}{|l|r|r|}
\hline Sector & studies metrics \\
\hline IHA & 4 & 3 \\
\hline LIHI & 5 & 0 \\
\hline FERC & 5 & 2 \\
\hline Peer- & & \\
Reviewed & & \\
Literature & 247 & 50 \\
\hline \multicolumn{1}{|r|}{ Totals } & 261 & 55 \\
\hline
\end{tabular}

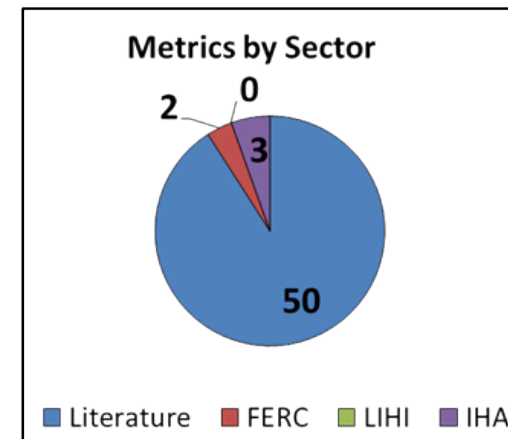

Metrics by Type

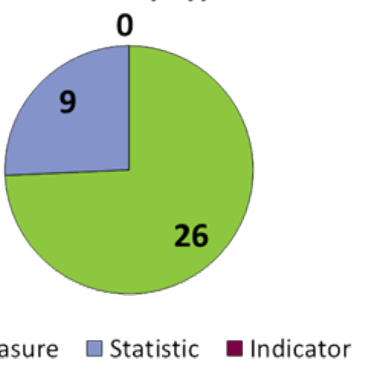

W2_Buffering capacity

Characteristics including $\mathrm{pH}$, alkalinity

\begin{tabular}{|l|l|}
\hline Metric Type & \multicolumn{1}{|c|}{ Metric Name } \\
\hline Measure & Alkalinity \\
\hline Measure & Auditing of $\mathrm{pH}$ \\
\hline Measure & $\begin{array}{l}\text { Baseline sampling of } \mathrm{pH} \text { in river and } \\
\text { tributaries }\end{array}$ \\
\hline Measure & ph \\
\hline Measure & pH groundwater \\
\hline Measure & pH reservoir outlet \\
\hline Measure & pH river \\
\hline Measure & pH river downstream \\
\hline Measure & Quarterly pH measurements \\
\hline Measure & Reservoir alkalinity \\
\hline Measure & Reservoir $\mathrm{pH}$ \\
\hline Measure & Stream alkalinity \\
\hline Measure & Stream pH \\
\hline Measure & Total alkalinity \\
\hline Measure & Total hardness \\
\hline Statistic & Conductivity epilimnion-- average \\
\hline Statistic & Conductivity hypolimnion-- average \\
\hline Statistic & Conductivity surface-- average \\
\hline Statistic & pH epilimnion-- average \\
\hline Statistic & pH hypolimnion-- average \\
\hline Statistic & pH surface-- average \\
\hline Statistic & $\begin{array}{l}\text { Total dissolved solids epilimnion--- } \\
\text { average }\end{array}$ \\
\hline Statistic & $\begin{array}{l}\text { Total dissolved solids hypolimnion-- } \\
\text { average }\end{array}$ \\
\hline Statistic & Total dissolved solids surface-- \\
average \\
\hline
\end{tabular}

ORNL Catalog of Environmental Metrics for Hydropower - 62 
3.6.3 W2_Dissolved Gasses

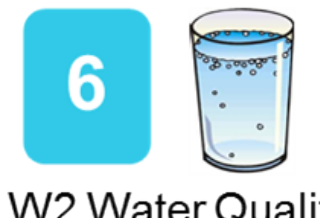

W2 Water Quality

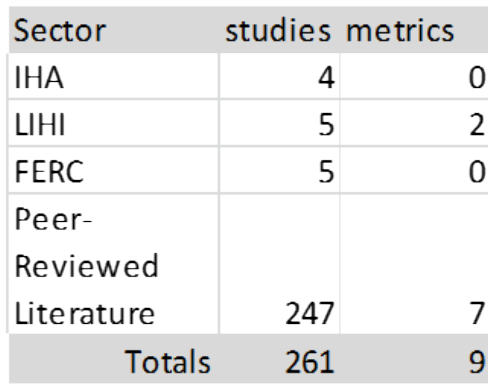
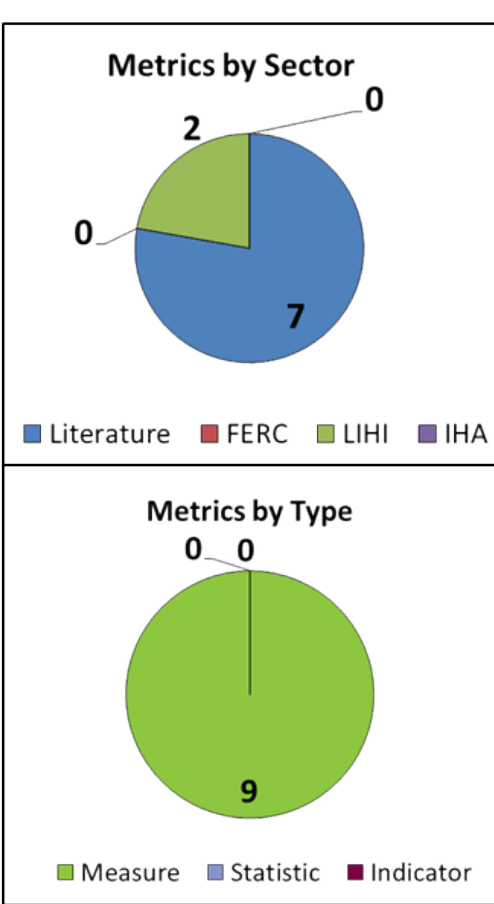

W2_Dissolved gasses

Concentration of non-greenhouse gases in water

\begin{tabular}{|l|l|}
\hline Metric Type & \multicolumn{1}{|c|}{ Metric Name } \\
\hline Measure & Carbon dioxide concentration in water \\
\hline Measure & Gas bubble trauma present \\
\hline Measure & Methane concentration in water \\
\hline Measure & Methane in surface water \\
\hline Measure & Reservoir DO \\
\hline Measure & Stream DO \\
\hline Measure & Total dissolved gas \\
\hline Measure & Total dissolved gases \\
\hline Measure & $\begin{array}{l}\text { Total dissolved gases-- percent } \\
\text { saturation }\end{array}$ \\
\hline
\end{tabular}




\subsubsection{W2_Dissovled Oxygen}

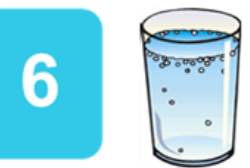

W2 Water Quality

\begin{tabular}{|l|r|r|}
\hline Sector & studies metrics \\
\hline IHA & 4 & 4 \\
\hline LIHI & 5 & 0 \\
\hline FERC & 5 & 7 \\
\hline Peer- & & \\
\hline Reviewed & & \\
Literature & 247 & 9 \\
\hline \multicolumn{1}{|r|}{ Totals } & 261 & 20 \\
\hline
\end{tabular}
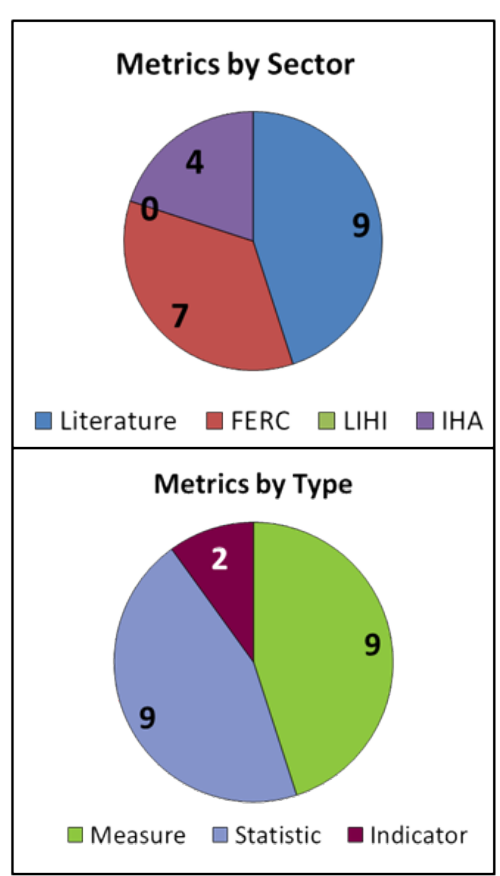

W2_Dissolved oxygen

Dissolved oxygen in water

\begin{tabular}{|c|c|}
\hline Metric Type & Metric Name \\
\hline Measure & Auditing of dissolved oxygen \\
\hline Measure & Dissolved oxygen \\
\hline Measure & Quarterly oxygen level measurements \\
\hline Measure & $\begin{array}{l}\text { Sediment interstitial water dissolved } \\
\text { oxygen }\end{array}$ \\
\hline Statistic & $\begin{array}{l}\text { Dissolved oxygen-- minimum average } \\
\text { daily }\end{array}$ \\
\hline Statistic & Dissolved oxygen profile \\
\hline Statistic & $\begin{array}{l}\text { Dissolved oxygen reservoir-- } \\
\text { minimum }\end{array}$ \\
\hline Statistic & Dissolved oxygen reservoir-- range \\
\hline Statistic & $\begin{array}{l}\text { Dissolved oxygen river downstream-- } \\
\text { range subdaily }\end{array}$ \\
\hline Statistic & $\begin{array}{l}\text { Dissolved oxygen river downtream-- } \\
\text { range }\end{array}$ \\
\hline Statistic & $\begin{array}{l}\text { Modeled downstream dissolved } \\
\text { oxygen levels }\end{array}$ \\
\hline Statistic & $\begin{array}{l}\text { Modeled reservoir dissolved oxygen } \\
\text { levels }\end{array}$ \\
\hline Statistic & $\begin{array}{l}\text { Sediment interstitial water dissolved } \\
\text { oxygen-- profile }\end{array}$ \\
\hline Indicator & $\begin{array}{l}\text { Dissolved oxygen longitudinal/ } \\
\text { development change }\end{array}$ \\
\hline Indicator & $\begin{array}{l}\text { Dissolved oxygen percent criteria } \\
\text { exceedance }\end{array}$ \\
\hline
\end{tabular}

ORNL Catalog of Environmental Metrics for Hydropower - 64 


\subsubsection{W2_Dissolved Oxygen}

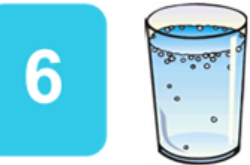

W2 Water Quality

\begin{tabular}{|l|r|r|}
\hline Sector & studies metrics \\
\hline IHA & 4 & 4 \\
\hline LIHI & 5 & 0 \\
\hline FERC & 5 & 7 \\
\hline Peer- & & \\
Reviewed & & \\
Literature & 247 & 9 \\
\hline \multicolumn{1}{|r|}{ Totals } & 261 & 20 \\
\hline
\end{tabular}

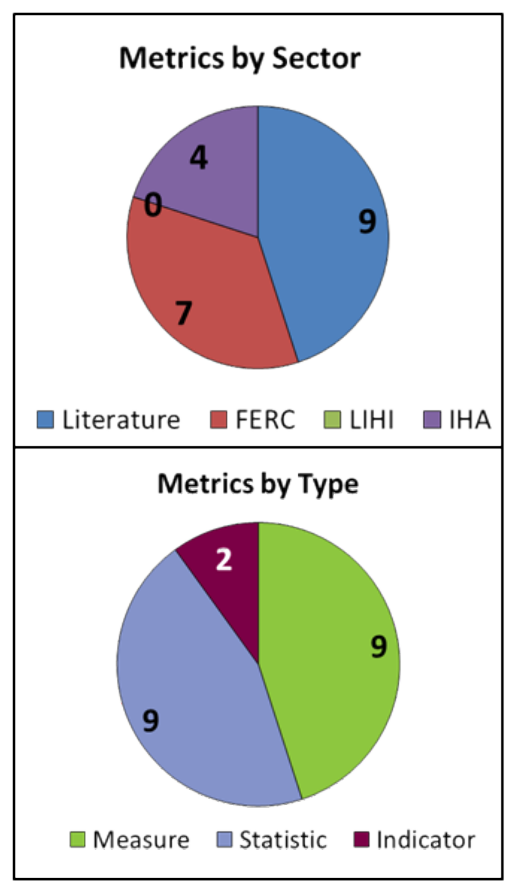

W2_Dissolved oxygen

\section{Dissolved oxygen in water}

\begin{tabular}{|l|l|}
\hline Metric Type & \multicolumn{1}{|c|}{ Metric Name } \\
\hline Measure & Auditing of dissolved oxygen \\
\hline Measure & Dissolved oxygen \\
\hline Measure & Quarterly oxygen level measurements \\
\hline Measure & $\begin{array}{l}\text { Sediment interstitial water dissolved } \\
\text { oxygen }\end{array}$ \\
\hline Statistic & $\begin{array}{l}\text { Dissolved oxygen-- minimum average } \\
\text { daily }\end{array}$ \\
\hline Statistic & Dissolved oxygen profile \\
\hline Statistic & $\begin{array}{l}\text { Dissolved oxygen reservoir-- } \\
\text { minimum }\end{array}$ \\
\hline Statistic & \begin{tabular}{l} 
Dissolved oxygen reservoir-- range \\
\hline Statistic
\end{tabular} $\begin{array}{l}\text { Dissolved oxygen river downstream-- } \\
\text { range subdaily }\end{array}$ \\
\hline Statistic & $\begin{array}{l}\text { Dissolved oxygen river downtream-- } \\
\text { range }\end{array}$ \\
\hline Statistic & $\begin{array}{l}\text { Modeled downstream dissolved } \\
\text { oxygen levels }\end{array}$ \\
\hline Statistic & $\begin{array}{l}\text { Modeled reservoir dissolved oxygen } \\
\text { levels }\end{array}$ \\
\hline Statistic & $\begin{array}{l}\text { Sediment interstitial water dissolved } \\
\text { oxygen-- profile }\end{array}$ \\
\hline Indicator & $\begin{array}{l}\text { Dissolved oxygen longitudinal/ } \\
\text { development change }\end{array}$ \\
\hline Indicator & $\begin{array}{l}\text { Dissolved oxygen percent criteria } \\
\text { exceedance }\end{array}$ \\
\hline
\end{tabular}




\subsubsection{W2_Ecosytem Function}

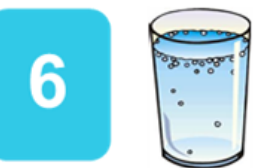

Ecosystem vital rates and processes, including gross primary productivity, respiration, biochemical oxygen demand

\section{W2 Water Quality}

\begin{tabular}{|l|r|r|}
\hline Sector & studies metrics \\
\hline IHA & 4 & 2 \\
\hline LIHI & 5 & 0 \\
\hline FERC & 5 & 1 \\
\hline Peer- & & \\
Reviewed & & \\
Literature & 247 & 10 \\
\hline \multicolumn{1}{|r|}{ Totals } & 261 & 13 \\
\hline
\end{tabular}

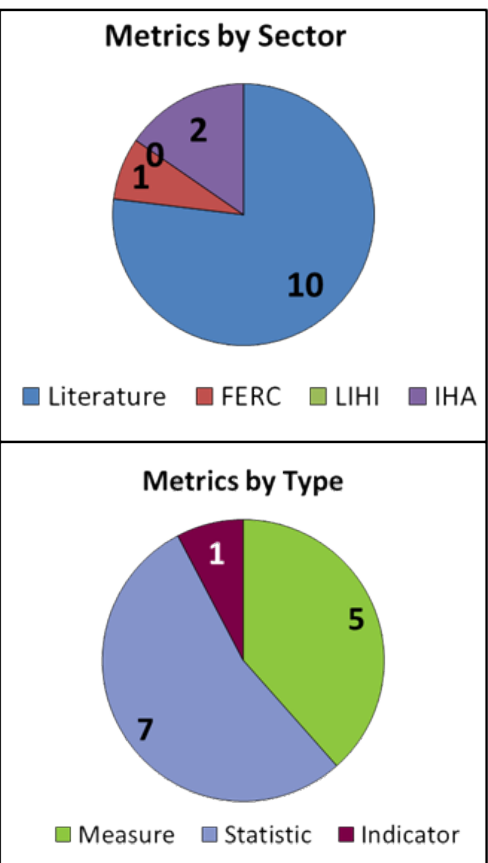

\begin{tabular}{|l|l|}
\hline Metric Type & \multicolumn{1}{|c|}{ Metric Name } \\
\hline Measure & Auditing of BOD \\
\hline Measure & $\begin{array}{l}\text { Baseline sampling of biological } \\
\text { oxygen demand in river and } \\
\text { tributaries }\end{array}$ \\
\hline Measure & Biological oxygen demand \\
\hline Measure & Chemical oxygen demand \\
\hline Statistic & $\begin{array}{l}\text { Biochemical oxygen demand } \\
\text { deoxygenation rate }\end{array}$ \\
\hline Statistic & $\begin{array}{l}\text { Fraction of dead phytoplankton } \\
\text { recycled to dissolved organic nitrogen }\end{array}$ \\
\hline Statistic & $\begin{array}{l}\text { Fraction of dead phytoplankton } \\
\text { recycled to dissolved organic } \\
\text { phosphorous }\end{array}$ \\
\hline Statistic & Mineralization rate \\
\hline Statistic & $\begin{array}{l}\text { Mineralization rate of dissolved } \\
\text { organic phosphorous }\end{array}$ \\
\hline Statistic & Nitrification rate \\
\hline Statistic & Sediment oxygen demand \\
\hline Indicator & Wetland function loss \\
\hline
\end{tabular}

ORNL Catalog of Environmental Metrics for Hydropower - 66 
3.6.7 W2_Gas Emissions

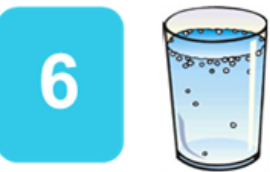

W2 Water Quality

\begin{tabular}{|l|r|r|}
\hline Sector & studies metrics \\
\hline IHA & 4 & 2 \\
\hline LIHI & 5 & 0 \\
\hline FERC & 5 & 0 \\
\hline Peer- & & \\
Reviewed & & \\
Literature & 247 & 28 \\
\hline \multicolumn{1}{|r|}{ Totals } & 261 & 30 \\
\hline
\end{tabular}

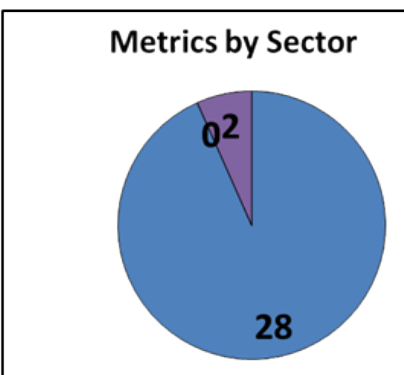

$\square$ Literature $\square$ FERC $\square \mathrm{LIHI} \quad \square \mathrm{IHA}$

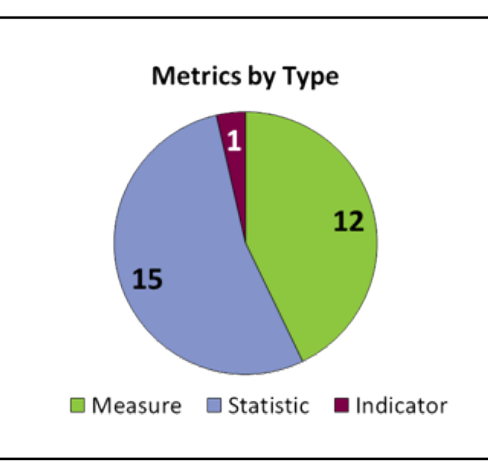

W2_Gas emissions

Concentration and ebullition of water-origin greenhouse gasses

\begin{tabular}{|l|l|}
\hline Metric Type & \\
\hline Measure & Carbon dioxide emissions-- air-water interface \\
\hline Measure & Carbon dioxide emissions at soil-water interface \\
\hline Measure & Carbon dioxide emissions-- average soil \\
\hline Measure & Carbon dioxide flux in air \\
\hline Measure & Methane emissions \\
\hline Measure & Methane emissions-- air-water interface \\
\hline Measure & Methane emissions at soil-water interface \\
\hline Measure & Methane emissions-- average soil \\
\hline Measure & Methane flux in air \\
\hline Measure & Spillway methane emissions \\
\hline Measure & Surface methane emissions \\
\hline Measure & Turbine methane emissions \\
\hline Statistic & Carbon dioxide emissions-- average flooded forest \\
\hline Statistic & Carbon dioxide emissions-- average flooded peatland \\
\hline Statistic & Carbon dioxide emissions-- average soil forest \\
\hline Statistic & Carbon dioxide emissions-- average soil peatland \\
\hline Statistic & Carbon dioxide emissions-- average soil-water interface forest \\
\hline Statistic & Carbon dioxide emissions-- average soil-water interface peatland \\
\hline Statistic & Greenhouse gas emissions \\
\hline Statistic & Methane emissions-- average flooded forest \\
\hline Statistic & Methane emissions-- average flooded peatland \\
\hline Statistic & Methane emissions-- average soil forest \\
\hline Statistic & Methane emissions-- average soil peatland \\
\hline Statistic & Methane emissions-- average soil-water interface forest \\
\hline Statistic & Methane emissions-- average soil-water interface peatland \\
\hline Statistic & Methane flux-- annual \\
\hline Statistic & Post-impoundment greenhouse gas emission \\
\hline Indicator & Environmental premium \\
\hline
\end{tabular}

Frequencies based on EMH Catalog Contents as of January 31, 2017

ORNL Catalog of Environmental Metrics for Hydropower - 67 

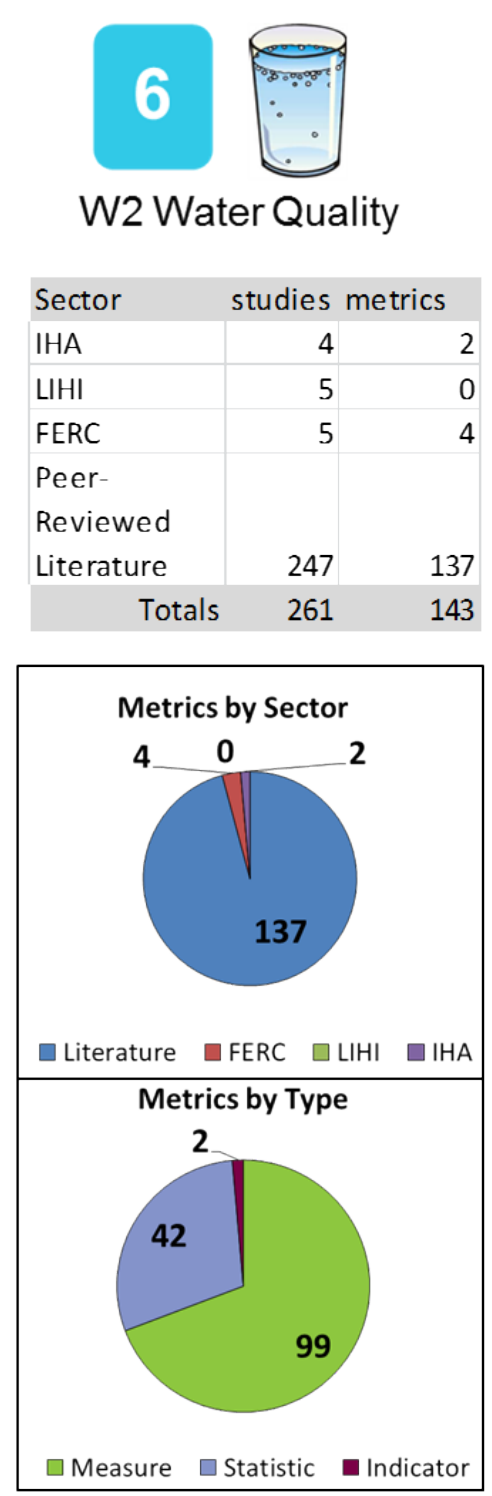

\section{W2_Nutrients}

All non-rare elements essential to life: nitrogen, phosphorous,

inorganic carbon, potassium, sulfur, and magnesium compounds (rare essential elements are included in "other elements")

\begin{tabular}{|l|l|}
\hline Metric Type & \multicolumn{1}{|c|}{ Metric Name } \\
\hline Measure & Algae bloom res ervoir present \\
\hline Measure & Ammonia \\
\hline Measure & Ammonium \\
\hline Measure & Ammoni um reservoir outlet \\
\hline Measure & Bank sediment-- calcium concentration \\
\hline Measure & Bank sediment-- chlorine concentration \\
\hline Measure & Bank sediment-- magnesium concentration \\
\hline Measure & Bank sediment-- phos phorous concentration \\
\hline Measure & Bank sediment-- potas sium concentration \\
\hline Measure & Bank sediment-- sodium concentration \\
\hline Measure & Bank sediment--sulfur concentration \\
\hline Measure & $\begin{array}{l}\text { Bas eline sampling of nutrients in river and } \\
\text { tributaries }\end{array}$ \\
\hline Measure & Calcium \\
\hline Measure & Calcium concentration groundwater \\
\hline Measure & Calcium concentration river \\
\hline Measure & Calcium concentrations sediment \\
\hline Measure & Carbon dioxide concentration river \\
\hline Measure & Dissolved inorganic nitrogen concentration \\
\hline Measure & Dissolved inorganic nitrogen res ervoir outlet \\
\hline Measure & $\begin{array}{l}\text { Dissolved inorganic phosphorous } \\
\text { concentration }\end{array}$ \\
\hline Measure & Magnesium concentration sediment \\
\hline Measure & Nitrate \\
\hline Measure & Nitrogen saturation below turbines \\
\hline Measure & Nitrogen saturation for ebay \\
\hline Measure & Nitrogen saturation forebay above turbines \\
\hline Measure & Nitrogen saturation stilling basin \\
\hline Measure & Nutrient levels reservoir \\
\hline Measure & Nutrient levels-- reservoir \\
\hline Measure & Organic nitrogen \\
\hline Measure & Organic phosphorous \\
\hline Measure & Orthophos phate \\
\hline
\end{tabular}

\begin{tabular}{|c|c|}
\hline Metric Type & Metric Name \\
\hline Measure & Particulate nitrogen reservoir outlet \\
\hline Measure & Phos phate \\
\hline Measure & Phos phorous concentration sediment \\
\hline Measure & Potassium concentration river \\
\hline Measure & Potassium concentration sediment \\
\hline Measure & Sodium \\
\hline Measure & Sodium oxate concentration \\
\hline Measure & Soluble reactive phos phorous \\
\hline Measure & Soluble reactive phos phorous res ervoir outlet \\
\hline Measure & Suspended sediment-- calcium concentration \\
\hline Measure & $\begin{array}{l}\text { Suspended s ediment-- magnesium } \\
\text { concentration }\end{array}$ \\
\hline Measure & $\begin{array}{l}\text { Suspended s ediment-- phosphorous } \\
\text { concentration }\end{array}$ \\
\hline Measure & $\begin{array}{l}\text { Suspended s ediment-- potassium } \\
\text { concentration }\end{array}$ \\
\hline Measure & Suspended s ediment-- sodium concentration \\
\hline Measure & Suspended sediment-- sulfur concentration \\
\hline Measure & Total dissolved nitrogen reservoir outl et \\
\hline Measure & Total Kjel dahl nitrogen \\
\hline Measure & Total nitrogen \\
\hline Measure & Total organic matter in sediment \\
\hline Measure & Total phosphorous \\
\hline Measure & Total phosphorous in sediment \\
\hline Measure & Total phosphorous inflow \\
\hline Measure & Total phosphorous outflow \\
\hline Measure & $\begin{array}{l}\text { Totalk Kjeldahl nitrogen minus ammonia/ } \\
\text { ammonium }\end{array}$ \\
\hline Measure & Water nutrients - Ammonium \\
\hline Measure & Water nutrients - Nitrate \\
\hline Measure & Water nutrients - Nitrite \\
\hline Measure & Water nutrients - SRP \\
\hline
\end{tabular}

Frequencies based on EMH Catalog Contents as of January 31, 2017

ORNL Catalog of Environmental Metrics for Hydropower - 68 
All non-rare elements essential to life: nitrogen, phosphorous, inorganic carbon, potassium, sulfur, and magnesium compounds (rare essential elements are included in "other elements")

\section{W2 Water Quality}

\begin{tabular}{|l|l|}
\hline Metric Type & \multicolumn{1}{|c|}{ Metric Name } \\
\hline Measure & Water nutrients - Total P \\
\hline Statistic & Ammonia epilimnion-- average \\
\hline Statistic & Ammonia hypolimnion-- average \\
\hline Statistic & Ammonia surface-- average \\
\hline Statistic & Bicarbonate epilimnion-- average \\
\hline Statistic & Bicarbonate hypolimnion-- average \\
\hline Statistic & Bicarbonate surface-- average \\
\hline Statistic & Calcium epilimnion-- average \\
\hline Statistic & Calcium hypolimnion-- average \\
\hline Statistic & Calcium surface-- average \\
\hline Statistic & Dissolved inorganic nitrogen load \\
\hline Statistic & Dissolved inorganic phosphorous Ioad \\
\hline Statistic & Magnesium epilimnion-- average \\
\hline Statistic & Magnesium hypolimnion-- average \\
\hline Statistic & Magnesium surface-- average \\
\hline Statistic & Net accumulation of phosphorous in reservoir \\
\hline Statistic & Nitrate epilimnion-- average \\
\hline Statistic & Nitrate hypolimnion-- average \\
\hline Statistic & Nitrate surface-- average \\
\hline Statistic & $\begin{array}{l}\text { Nitrogen saturation below turbines-- average } \\
\text { daily }\end{array}$ \\
\hline Statistic & $\begin{array}{l}\text { Nitrogen saturation below turbines-- average } \\
\text { seasonal }\end{array}$ \\
\hline Statistic & $\begin{array}{l}\text { Nitrogen saturation forebay above spillway-- } \\
\text { average daily }\end{array}$ \\
\hline Statistic & $\begin{array}{l}\text { Nitrogen saturation forebay above spillway-- } \\
\text { average seasonal }\end{array}$ \\
\hline Statistic & $\begin{array}{l}\text { Nitrogen saturation for ebay above turbines-- } \\
\text { average daily }\end{array}$ \\
\hline Statistic & $\begin{array}{l}\text { Nitrogen saturation for ebay above turbines-- } \\
\text { average s easonal }\end{array}$ \\
\hline
\end{tabular}

\begin{tabular}{|l|l|}
\hline Metric Type & \multicolumn{1}{|c|}{ Metric Name } \\
\hline Statistic & $\begin{array}{l}\text { Nitrogen saturation stilling basin-- average } \\
\text { daily }\end{array}$ \\
\hline Statistic & $\begin{array}{l}\text { Nitrogen saturation stilling basin-- average } \\
\text { seasonal }\end{array}$ \\
\hline Statistic & Percent by weight carbonate fraction \\
\hline Statistic & Phos phate epilimnion-- average \\
\hline Statistic & Phos phate hypolimnion-- average \\
\hline Statistic & Phosphate surface-- average \\
\hline Statistic & Potassium to gallium ratio \\
\hline Statistic & $\begin{array}{l}\text { Ratio of strontium 87 isotope to strontium 86 } \\
\text { isotope }\end{array}$ \\
\hline Statistic & Sodium epilimnion-- average \\
\hline Statistic & Sodium hypolimnion-- average \\
\hline Statistic & Sodium surface-- average \\
\hline Statistic & Sulfate epilimnion-- average \\
\hline Statistic & Sulfate hypolimnion-- average \\
\hline Statistic & Sulfate surface-- average \\
\hline Indicator & Nutrient levels construction discharge \\
\hline Indicator & Nutrient monitoring \\
\hline
\end{tabular}

ORNL Catalog of Environmental Metrics for Hydropower - 69 
3.6.9 W2_Organic Material

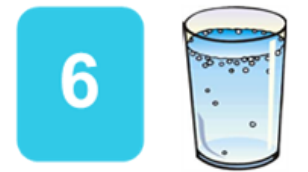

W2 Water Quality

\begin{tabular}{|l|rr|}
\hline Sector & studies metrics \\
\hline IHA & 4 & 2 \\
\hline LIHI & 5 & 0 \\
\hline FERC & 5 & 0 \\
\hline Peer- & & \\
Reviewed & & \\
\hline Literature & 247 & 7 \\
\hline \multicolumn{1}{r|}{ Totals } & 261 & 9 \\
\hline
\end{tabular}

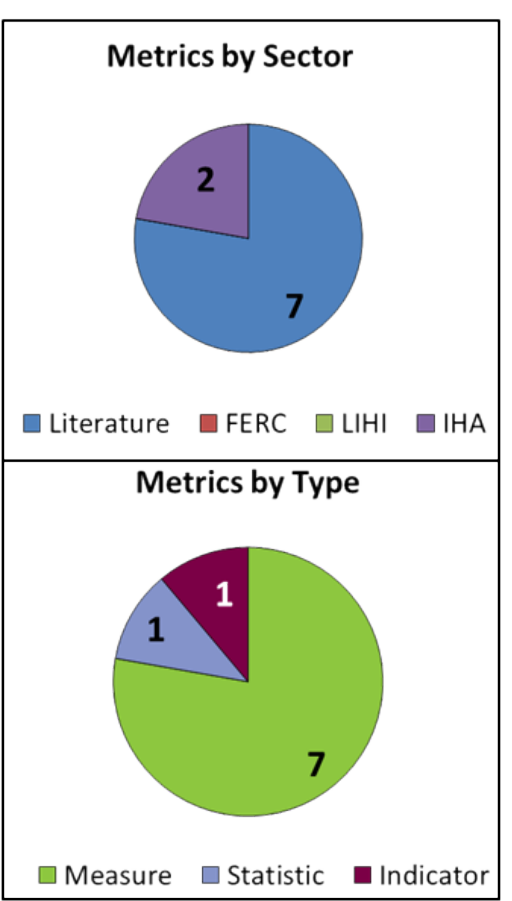

W2_Organic material

Dissolved organic carbon and other organic non-pollutants

\begin{tabular}{|l|l|}
\hline Metric Type & \multicolumn{1}{|c|}{ Metric Name } \\
\hline Measure & Chromorphic organic matter \\
\hline Measure & Dissolved Organic Carbon \\
\hline Measure & Organic siltation \\
\hline Measure & Total organic matter \\
\hline Statistic & Modeled reservoir carbon load \\
\hline Indicator & Baseline microbiological parameters \\
\hline
\end{tabular}




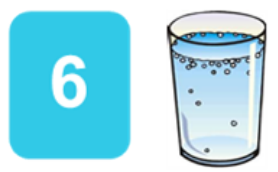

W2 Water Quality

\begin{tabular}{|l|r|r|}
\hline Sector & studies metrics \\
\hline IHA & 4 & 0 \\
\hline LIHI & 5 & 0 \\
\hline FERC & 5 & 0 \\
\hline Peer- & & \\
Reviewed & & \\
Literature & 247 & 470 \\
\hline \multicolumn{1}{|r|}{ Totals } & 261 & 470 \\
\hline
\end{tabular}

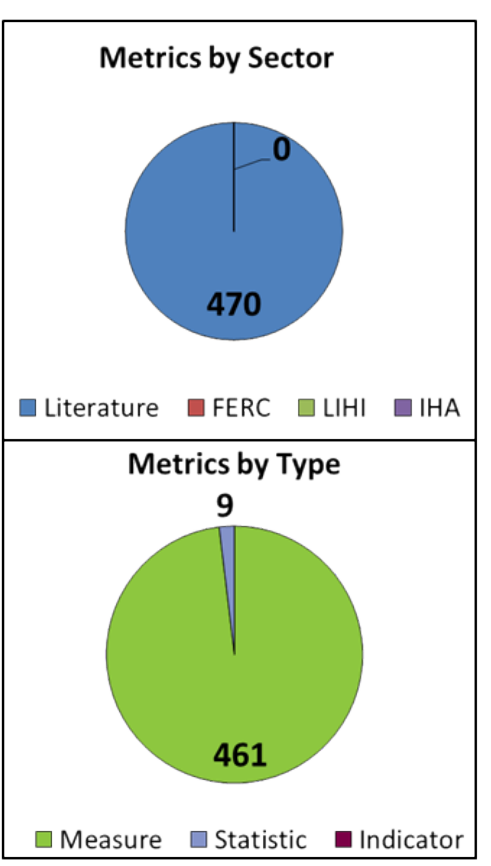

W2_Other elements
Elements and compounds that are not listed on the EPA Toxic and Priority Pollutants list

\begin{tabular}{|c|c|}
\hline Metric Type & Metric Name \\
\hline Measure & $\begin{array}{l}\text { Bank sediment-- antimony } \\
\text { concentration }\end{array}$ \\
\hline Measure & Bank sediment-- barium concentration \\
\hline Measure & $\begin{array}{l}\text { Bank sediment-- beryllium } \\
\text { concentration }\end{array}$ \\
\hline Measure & $\begin{array}{l}\text { Bank sediment-- bis muth } \\
\text { concentration }\end{array}$ \\
\hline Measure & Bank sediment-- boron concentration \\
\hline Measure & $\begin{array}{l}\text { Bank s ediment-- bromium } \\
\text { concentration }\end{array}$ \\
\hline Measure & $\begin{array}{l}\text { Bank sediment-- cadmium } \\
\text { concentration }\end{array}$ \\
\hline Measure & Bank sediment-- cerium concentration \\
\hline Measure & $\begin{array}{l}\text { Bank sediment-- chromium } \\
\text { concentration }\end{array}$ \\
\hline Measure & Bank sediment-- cobalt concentration \\
\hline Measure & Bank sediment-- copper concentration \\
\hline Measure & $\begin{array}{l}\text { Bank sediment-- dysprosium } \\
\text { concentration }\end{array}$ \\
\hline Measure & Bank sediment-- erbium concentration \\
\hline Measure & $\begin{array}{l}\text { Bank sediment-- europium } \\
\text { concentration }\end{array}$ \\
\hline Measure & $\begin{array}{l}\text { Bank sediment-- gadolinium } \\
\text { concentration }\end{array}$ \\
\hline Measure & $\begin{array}{l}\text { Bank sediment-- gallium } \\
\text { concentration }\end{array}$ \\
\hline Measure & Bank sediment-- gold concentration \\
\hline Measure & $\begin{array}{l}\text { Bank sediment-- hafnium } \\
\text { concentration }\end{array}$ \\
\hline
\end{tabular}

\begin{tabular}{|l|l|}
\hline Metric Type & \multicolumn{1}{|c|}{ Metric Name } \\
\hline Measure & $\begin{array}{l}\text { Bank sediment-- holmium } \\
\text { concentration }\end{array}$ \\
\hline Measure & Bank sediment-- iron concentration \\
\hline Measure & $\begin{array}{l}\text { Bank sediment-- Ianthanum } \\
\text { concentration }\end{array}$ \\
\hline Measure & Bank sediment-- lithium concentration \\
\hline Measure & $\begin{array}{l}\text { Bank sediment-- Iutetium } \\
\text { concentration }\end{array}$ \\
\hline Measure & $\begin{array}{l}\text { Bank sediment-- magnesium } \\
\text { concentration }\end{array}$ \\
\hline Measure & $\begin{array}{l}\text { Bank sediment-- mananese } \\
\text { concentration }\end{array}$ \\
\hline Measure & $\begin{array}{l}\text { Bank sediment-- molybdenum } \\
\text { concentration }\end{array}$ \\
\hline Measure & $\begin{array}{l}\text { Bank sediment-- neodymium } \\
\text { concentration }\end{array}$ \\
\hline Measure & Bank sediment-- nickel concentration \\
\hline Measure & $\begin{array}{l}\text { Bank sediment-- niobium } \\
\text { concentration }\end{array}$ \\
\hline Measure & $\begin{array}{l}\text { Bank sediment-- platinum } \\
\text { concentration }\end{array}$ \\
\hline Measure & $\begin{array}{l}\text { Bank sediment-- praseodymium } \\
\text { concentration }\end{array}$ \\
\hline Measure & $\begin{array}{l}\text { Bank sediment-- rubidium } \\
\text { concentration }\end{array}$ \\
\hline Measure & $\begin{array}{l}\text { Bank sediment-- samarium } \\
\text { concentration }\end{array}$ \\
\hline Measure & $\begin{array}{l}\text { Bank sediment-- scandium } \\
\text { concentration }\end{array}$ \\
\hline Bank sediment-- silicon concentration \\
\hline Beasure & Bank sediment-- silver concentration \\
\hline Mand
\end{tabular}

Frequencies based on EMH Catalog Contents as of January 31, 2017

ORNL Catalog of Environmental Metrics for Hydropower - 71 


\section{W2 Water Quality}

\begin{tabular}{|c|c|}
\hline Metric Type & Metric Name \\
\hline Measure & $\begin{array}{l}\text { Bank sediment-- strontium } \\
\text { concentration }\end{array}$ \\
\hline Measure & $\begin{array}{l}\text { Bank sediment-- tantalum } \\
\text { concentration }\end{array}$ \\
\hline Measure & $\begin{array}{l}\text { Bank sediment-- terbium } \\
\text { concentration }\end{array}$ \\
\hline Measure & $\begin{array}{l}\text { Bank sediment-- thallium } \\
\text { concentration }\end{array}$ \\
\hline Measure & $\begin{array}{l}\text { Bank sediment-- thorium } \\
\text { concentration }\end{array}$ \\
\hline Measure & $\begin{array}{l}\text { Bank sediment-- thulium } \\
\text { concentration }\end{array}$ \\
\hline Measure & Bank sediment-- tin concentration \\
\hline Measure & $\begin{array}{l}\text { Bank sediment-- titanium } \\
\text { concentration }\end{array}$ \\
\hline Measure & $\begin{array}{l}\text { Bank sediment-- tungsten } \\
\text { concentration }\end{array}$ \\
\hline Measure & $\begin{array}{l}\text { Bank sediment-- uranium } \\
\text { concentration }\end{array}$ \\
\hline Measure & $\begin{array}{l}\text { Bank sediment-- vanadium } \\
\text { concentration }\end{array}$ \\
\hline Measure & $\begin{array}{l}\text { Bank s ediment-- ytterbium } \\
\text { concentration }\end{array}$ \\
\hline Measure & Bank sediment-- yttrium concentration \\
\hline Measure & Bank sediment-- zinc concentration \\
\hline Measure & $\begin{array}{l}\text { Bank sediment-- zirconium } \\
\text { concentration }\end{array}$ \\
\hline Measure & Chloride \\
\hline Measure & Chromium \\
\hline Measure & Dissolved iron reservoir outl et \\
\hline Measure & Dissolved silica concentration \\
\hline
\end{tabular}

\begin{tabular}{|c|c|}
\hline Metric Type & Metric Name \\
\hline Measure & Sediment-- rubidium concentration \\
\hline Measure & Sediment-- silicon concentration \\
\hline Measure & Sediment-- sodium concentration \\
\hline Measure & Sediment-- strontium concentration \\
\hline Measure & Sediment-- titanium concentration \\
\hline Measure & Sediment-- vanadium concentration \\
\hline Measure & Sediment-- zinc concentration \\
\hline Measure & Sediment-- zirconium concentration \\
\hline Measure & Silicate \\
\hline Measure & Sulfate \\
\hline Measure & $\begin{array}{l}\text { Suspended sediment-- antimony } \\
\text { concentration }\end{array}$ \\
\hline Measure & $\begin{array}{l}\text { Suspended sediment-- arsenic } \\
\text { concentration }\end{array}$ \\
\hline Measure & $\begin{array}{l}\text { Suspended sediment-- barium } \\
\text { concentration }\end{array}$ \\
\hline Measure & $\begin{array}{l}\text { Suspended sediment-- beryllium } \\
\text { concentration }\end{array}$ \\
\hline Measure & $\begin{array}{l}\text { Suspended sediment-- bis muth } \\
\text { concentration }\end{array}$ \\
\hline Measure & $\begin{array}{l}\text { Suspended sediment-- boron } \\
\text { concentration }\end{array}$ \\
\hline Measure & $\begin{array}{l}\text { Suspended sediment-- bromium } \\
\text { concentration }\end{array}$ \\
\hline Measure & $\begin{array}{l}\text { Suspended sediment-- cadmium } \\
\text { concentration }\end{array}$ \\
\hline Measure & $\begin{array}{l}\text { Suspended sediment-- cerium } \\
\text { concentration }\end{array}$ \\
\hline Measure & $\begin{array}{l}\text { Suspended sediment-- chromium } \\
\text { concentration }\end{array}$ \\
\hline $\begin{array}{l}\text { Measure } \\
\text { wer - } 72\end{array}$ & $\begin{array}{l}\text { Suspended sediment-- cobalt } \\
\text { concentration }\end{array}$ \\
\hline
\end{tabular}


Page 3 of 4

\section{W2 Water Quality}

\begin{tabular}{|c|c|}
\hline Metric Type & Metric Name \\
\hline Measure & $\begin{array}{l}\text { Suspended sediment-- copper } \\
\text { concentration }\end{array}$ \\
\hline Measure & $\begin{array}{l}\text { Suspended sediment-- dys prosium } \\
\text { concentration }\end{array}$ \\
\hline Measure & $\begin{array}{l}\text { Suspended sediment-- erbium } \\
\text { concentration }\end{array}$ \\
\hline Measure & $\begin{array}{l}\text { Suspended sediment-- europium } \\
\text { concentration }\end{array}$ \\
\hline Measure & $\begin{array}{l}\text { Suspended sediment-- gadolinium } \\
\text { concentration }\end{array}$ \\
\hline Measure & $\begin{array}{l}\text { Suspended sediment-- gallium } \\
\text { concentration }\end{array}$ \\
\hline Measure & $\begin{array}{l}\text { Suspended sediment-- gold } \\
\text { concentration }\end{array}$ \\
\hline Measure & $\begin{array}{l}\text { Suspended sediment-- hafnium } \\
\text { concentration }\end{array}$ \\
\hline Measure & $\begin{array}{l}\text { Suspended sediment-- holmium } \\
\text { concentration }\end{array}$ \\
\hline Measure & $\begin{array}{l}\text { Suspended sediment-- iron } \\
\text { concentration }\end{array}$ \\
\hline Measure & $\begin{array}{l}\text { Suspended sediment-- lanthanum } \\
\text { concentration }\end{array}$ \\
\hline Measure & $\begin{array}{l}\text { Suspended sediment-- lithium } \\
\text { concentration }\end{array}$ \\
\hline Measure & $\begin{array}{l}\text { Suspended sediment-- lutetium } \\
\text { concentration }\end{array}$ \\
\hline Measure & $\begin{array}{l}\text { Suspended sediment-- mananese } \\
\text { concentration }\end{array}$ \\
\hline Measure & $\begin{array}{l}\text { Suspended sediment-- molybdenum } \\
\text { concentration }\end{array}$ \\
\hline Measure & $\begin{array}{l}\text { Suspended sediment-- neodymium } \\
\text { concentration }\end{array}$ \\
\hline
\end{tabular}

\begin{tabular}{|c|c|}
\hline Metric Type & Metric Name \\
\hline Measure & $\begin{array}{l}\text { Suspended sediment-- nickel } \\
\text { concentration }\end{array}$ \\
\hline Measure & $\begin{array}{l}\text { Suspended sediment-- niobium } \\
\text { concentration }\end{array}$ \\
\hline Measure & $\begin{array}{l}\text { Suspended sediment-- phosphorous } \\
\text { concentration }\end{array}$ \\
\hline Measure & $\begin{array}{l}\text { Suspended sediment-- platinum } \\
\text { concentration }\end{array}$ \\
\hline Measure & $\begin{array}{l}\text { Suspended sediment-- praseodymi um } \\
\text { concentration }\end{array}$ \\
\hline Measure & $\begin{array}{l}\text { Suspended sediment-- rubidium } \\
\text { concentration }\end{array}$ \\
\hline Measure & $\begin{array}{l}\text { Suspended sediment-- samarium } \\
\text { concentration }\end{array}$ \\
\hline Measure & $\begin{array}{l}\text { Suspended sediment-- scandium } \\
\text { concentration }\end{array}$ \\
\hline Measure & $\begin{array}{l}\text { Suspended sediment-- selenium } \\
\text { concentration }\end{array}$ \\
\hline Measure & $\begin{array}{l}\text { Suspended sediment-- silicon } \\
\text { concentration }\end{array}$ \\
\hline Measure & $\begin{array}{l}\text { Suspended sediment-- silver } \\
\text { concentration }\end{array}$ \\
\hline Measure & $\begin{array}{l}\text { Suspended sediment-- sodium } \\
\text { concentration }\end{array}$ \\
\hline Measure & $\begin{array}{l}\text { Suspended sediment-- strontium } \\
\text { concentration }\end{array}$ \\
\hline Measure & $\begin{array}{l}\text { Suspended sediment-- tantalum } \\
\text { concentration }\end{array}$ \\
\hline Measure & $\begin{array}{l}\text { Suspended sediment-- terbium } \\
\text { concentration }\end{array}$ \\
\hline $\begin{array}{l}\text { Measure } \\
\text { RNL Catalo }\end{array}$ & $\begin{array}{l}\text { Suspended sediment-- thallium } \\
\text { gof Environmental Metrics for Hydr } \\
\text { concentration }\end{array}$ \\
\hline
\end{tabular}

\begin{tabular}{|l|l|}
\hline Metric Type & \multicolumn{1}{|c|}{ Metric Name } \\
\hline Measure & $\begin{array}{l}\text { Suspended sediment-- thorium } \\
\text { concentration }\end{array}$ \\
\hline Measure & $\begin{array}{l}\text { Suspended sediment-- thulium } \\
\text { concentration }\end{array}$ \\
\hline Measure & $\begin{array}{l}\text { Suspended sediment-- tin } \\
\text { concentration }\end{array}$ \\
\hline Measure & $\begin{array}{l}\text { Suspended sediment-- titanium } \\
\text { concentration }\end{array}$ \\
\hline Measure & $\begin{array}{l}\text { Suspended sediment-- tungsten } \\
\text { concentration }\end{array}$ \\
\hline Measure & $\begin{array}{l}\text { Suspended sediment-- uranium } \\
\text { concentration }\end{array}$ \\
\hline Measure & $\begin{array}{l}\text { Suspended sediment-- vanadium } \\
\text { concentration }\end{array}$ \\
\hline Measure & $\begin{array}{l}\text { Suspended sediment-- ytterbium } \\
\text { concentration }\end{array}$ \\
\hline Measure & $\begin{array}{l}\text { Suspended sediment-- yttrium } \\
\text { concentration }\end{array}$ \\
\hline Measure & $\begin{array}{l}\text { Suspended sediment-- zinc } \\
\text { concentration }\end{array}$ \\
\hline Measure & $\begin{array}{l}\text { Suspended sediment-- zirconium } \\
\text { concentration }\end{array}$ \\
\hline Measure & Upwelling irradiance \\
\hline Measure & $\begin{array}{l}\text { Water-- bicarbonate concentration } \\
\text { river }\end{array}$ \\
\hline Measure & Water-- cadmium concentration \\
\hline Measure & Water el ements - Chloride \\
\hline Measure & Water-- sodium concentration river \\
\hline Staterstic
\end{tabular}




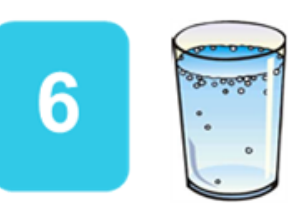

Elements and compounds that are not listed on the EPA Toxic and Priority Pollutants list

\section{W2 Water Quality}

\begin{tabular}{|l|l|}
\hline Metric Type & \multicolumn{1}{|c|}{ Metric Name } \\
\hline Statistic & Iron epilimnion-- average \\
\hline Statistic & Iron hypolimnion-- average \\
\hline Statistic & Iron surface-- average \\
\hline Statistic & Manganese epilimnion-- average \\
\hline Statistic & Manganese hypolimnion-- average \\
\hline Statistic & Manganese surface-- average \\
\hline Statistic & $\begin{array}{l}\text { Ratio of strontium } 87 \text { isotope to } \\
\text { strontium 86 isotope }\end{array}$ \\
\hline
\end{tabular}


3.6.11 W2_Pollutants

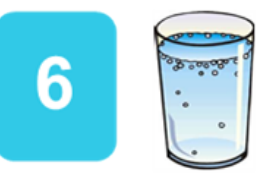

W2 Water Quality

\begin{tabular}{|l|r|r|}
\hline Sector & studies metrics \\
\hline IHA & 4 & 11 \\
\hline LIHI & 5 & 0 \\
\hline FERC & 5 & 3 \\
\hline Peer- & & \\
Reviewed & & \\
\hline Literature & 247 & 57 \\
\hline \multicolumn{1}{|r|}{ Totals } & 261 & 71 \\
\hline
\end{tabular}

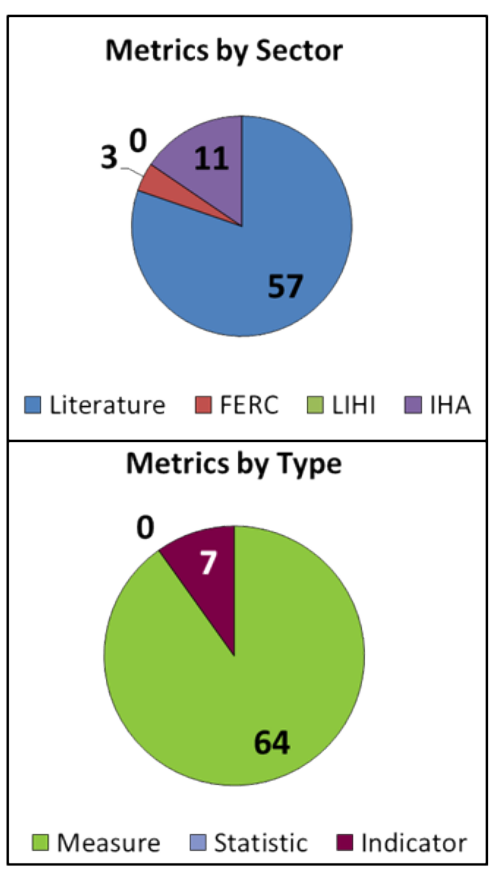

W2_Pollutants
Pollutants listed on the EPA Toxic and Priority Pollutants list that are not included in other EMH categories

\begin{tabular}{|l|l|}
\hline Metric Type & \multicolumn{1}{|c|}{ Metric Name } \\
\hline Measure & Bacterial concentration \\
\hline Measure & Bank sediment-- al umi num concentration \\
\hline Measure & Bank sediment-- ars enic concentration \\
\hline Measure & Bank sediment-- lead concentration \\
\hline Measure & Bank sediment-- sel enium concentration \\
\hline Measure & $\begin{array}{l}\text { Bas eline sampling of toxic and priority } \\
\text { pollutants in river and tributaries }\end{array}$ \\
\hline Measure & Coliform counts \\
\hline Measure & E coli \\
\hline Measure & E. coli monitoring \\
\hline Measure & Fecal coliform \\
\hline Measure & Lead \\
\hline Measure & Lead concentration--sediment \\
\hline Measure & Lead concentration--water \\
\hline Measure & $\begin{array}{l}\text { Monthly water quality tests against } \\
\text { drinking water standards }\end{array}$ \\
\hline Measure & Oil and grease \\
\hline Measure & Organic pollution \\
\hline Measure & Sediment-- ars enic concentration \\
\hline Measure & Sediment-- mercury concentration \\
\hline Measure & $\begin{array}{l}\text { Suspended sediment-- al uminum } \\
\text { concentration }\end{array}$ \\
\hline Measure & $\begin{array}{l}\text { Suspended sediment-- ars enic } \\
\text { concentration }\end{array}$ \\
\hline Measure & Suspended sediment-- lead concentration \\
\hline Measure & $\begin{array}{l}\text { Suspended s ediment-- selenium } \\
\text { concentration }\end{array}$ \\
\hline Measure & Test for industrial effluent limits \\
\hline Measure & Total coliform \\
\hline Measure & Toxic substances \\
\hline Measure & Water-- ars enic concentration \\
\hline Measure & Water-- mercury concentration \\
\hline Indicator & $\begin{array}{l}\text { Bacterial concentration construction } \\
\text { discharge }\end{array}$ \\
\hline
\end{tabular}

\begin{tabular}{|l|l|}
\hline Metric Type & \multicolumn{1}{|c|}{ Metric Name } \\
\hline Indicator & Baseline physical \& chemical parameters \\
\hline Indicator & Baseline water quality \\
\hline Indicator & CCME Water Quality Index \\
\hline Indicator & $\begin{array}{l}\text { Ongoing, comprehensive water quality } \\
\text { monitoring }\end{array}$ \\
\hline Indicator & Toxic construction discharge \\
\hline Indicator & Water quality index score \\
\hline
\end{tabular}

ORNL Catalog of Environmental Metrics for Hydropower - 75 
3.6.12 W2_Solid Transport, Turbidity \& Conductivity

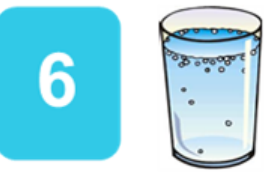

W2 Water Quality

\begin{tabular}{|l|r|r|}
\hline Sector & studies metrics \\
\hline IHA & 4 & 5 \\
\hline LIHI & 5 & 0 \\
\hline FERC & 5 & 7 \\
\hline Peer- & & \\
Reviewed & & \\
Literature & 247 & 58 \\
\hline \multicolumn{1}{|r|}{ Totals } & 261 & 70 \\
\hline
\end{tabular}

Metrics by Sector

0

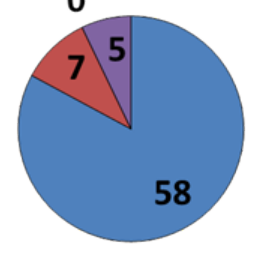

$\square$ Literature $\square$ FERC $\square$ LIHI $\square$ IHA

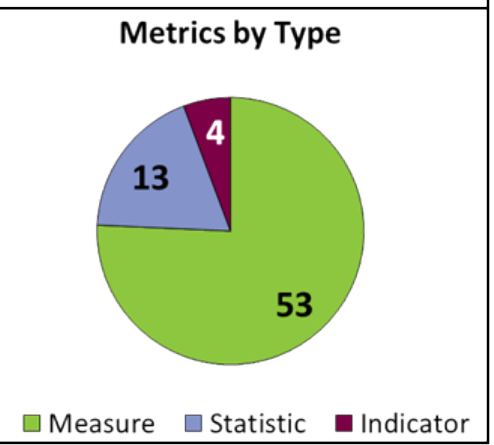

W2_Solid transport, turbidity \& conductivity
Descriptions of dissolved and suspended solids in water such as turbidity, suspended or dissolved solids, conductance

\begin{tabular}{|l|l|}
\hline Metric Type & \multicolumn{1}{|c|}{ Metric Name } \\
\hline Measure & $\begin{array}{l}\text { Baseline sampling of el ectrical } \\
\text { conductivity, dissolved solids and TSS in } \\
\text { river and tributaries }\end{array}$ \\
\hline Measure & Color \\
\hline Measure & Conductivity \\
\hline Measure & Conductivity river downstream \\
\hline Measure & Continuous sediment load monitoring \\
\hline Measure & Quarterly conductivity measurements \\
\hline Measure & Res ervoir conductivity \\
\hline Measure & Res ervoir secchi depth \\
\hline Measure & Salinity \\
\hline Measure & Salinity surface \\
\hline Measure & Secchi depth \\
\hline Measure & Sediment loading reservoir \\
\hline Measure & Specific conductance \\
\hline Measure & Stream conductivity \\
\hline Measure & Stream secchi depth \\
\hline Measure & Sus pended sediment \\
\hline Measure & Suspended sediment discharge \\
\hline Measure & Suspended sediment sampling \\
\hline Measure & Suspended solids \\
\hline Measure & $\begin{array}{l}\text { Total dissolved soilds upper layer of } \\
\text { reservoir water }\end{array}$ \\
\hline Measure & Total dissolved solids \\
\hline Measure & Total dissolved solids concentration river \\
\hline Measure & Total dissolved solids in groundwater \\
\hline Measure & $\begin{array}{l}\text { Total dissolved solids l ower layer of } \\
\text { reservoir water }\end{array}$ \\
\hline Measure & $\begin{array}{l}\text { Total dissolved solids middle layer of } \\
\text { res ervoir water }\end{array}$ \\
\hline
\end{tabular}

ORNL Catalog of Environmental Metrics for Hydropower - 76 


\subsection{BB_Biota and Biodiversity}

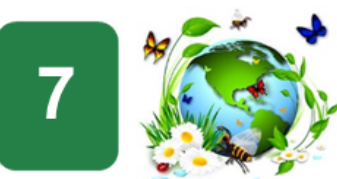

BB Biota and Biodiversity

\begin{tabular}{|l|r|r|}
\hline Sector & \# of studies & \# of metrics \\
\hline IHA & 4 & 21 \\
\hline LIHI & 5 & 24 \\
\hline FERC & 5 & 134 \\
\hline $\begin{array}{l}\text { Peer-Reviewed } \\
\text { Literature }\end{array}$ & 247 & 333 \\
\hline \multicolumn{1}{|r|}{ Totals } & 261 & 512 \\
\hline
\end{tabular}

Biota parameter frequency at study-level (extraction table)

Primary

Parameter

Biota/ BB_Abundance, density

biodiversity BB_Behavior, movement

BB_Colonization, extinction

BB_Demographics, age, sex, size

ition, reproduction, mortality

政

BB_Fuction

作

BB_Internal composition nutrients abnermalities

B _tifenistory trait characteristics

type measures

WQ_Algae, primary productivity

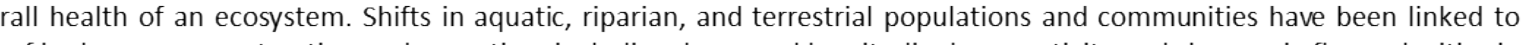
tation of uplands upstream of dams, changes in groundwater depth both up- and downstream of dams, and changes in sediment and flow regimes. Identifying metrics to accurately assess population and community changes is essential to understanding the

Spatial scales for biota represented at study-level (extraction table)

\section{Metrics by Sector}

2421

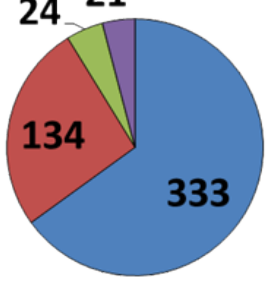

$\square$ Literature

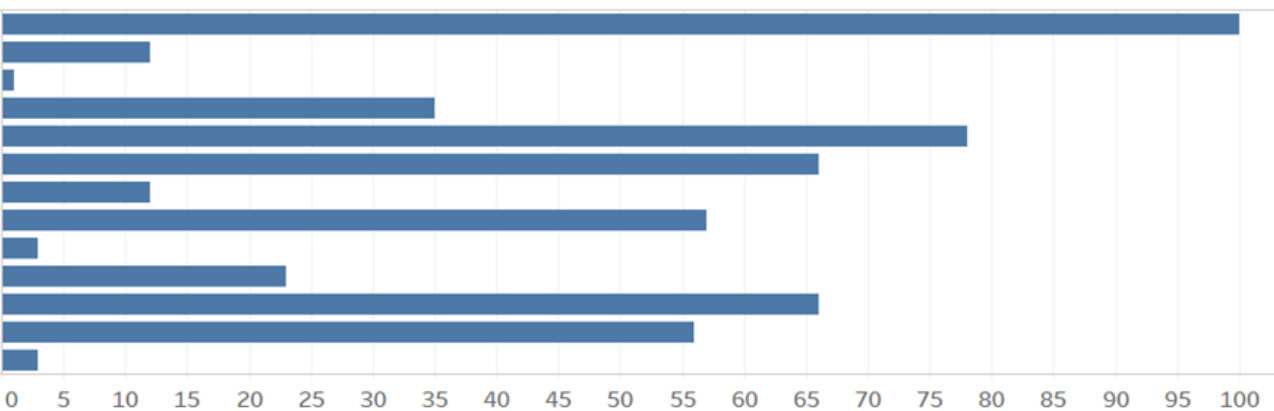

\begin{tabular}{ll}
$\begin{array}{l}\text { Primary } \\
\text { Category }\end{array}$ & Spatscale \\
\hline Biota/ & Basin \\
biodiversity & Dam \\
& Dam Cascade \\
& Landscape \\
& Project \\
& Reach \\
& Reservoir \\
& River_downstream \\
& River_upstream \\
& Within dam
\end{tabular}

$$
=
$$

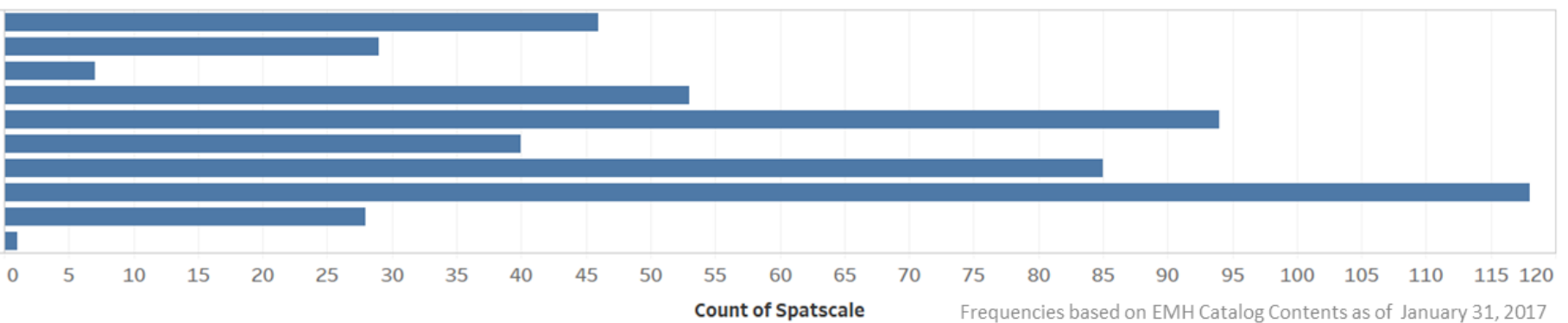

ORNL Catalog of Environmental Metrics for Hydropower - 77 


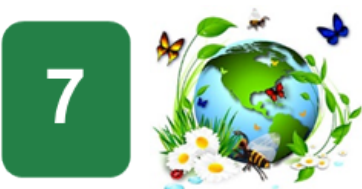

BB Biota and

Biodiversity

\begin{tabular}{|c|c|c|}
\hline Sector & studies & metrics \\
\hline IHA & 4 & 5 \\
\hline LIHI & 5 & 0 \\
\hline FERC & 5 & 8 \\
\hline $\begin{array}{l}\text { Peer-Reviewed } \\
\text { Literature }\end{array}$ & 247 & 87 \\
\hline Totals & 261 & 100 \\
\hline
\end{tabular}

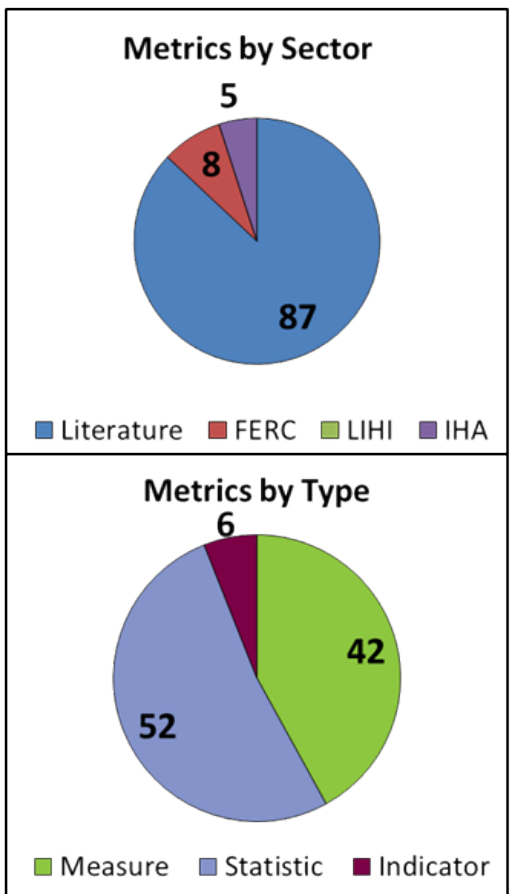

\section{BB_Abundance, density}

\begin{tabular}{|l|l|}
\hline Metric Type & \multicolumn{1}{|c|}{ Metric Name } \\
\hline Measure & Basal cover \\
\hline Measure & Benthic food availability \\
\hline Measure & Bird abundance \\
\hline Measure & Cyanobacteria abundance \\
\hline Measure & Diatom abundance \\
\hline Measure & Fish abundance \\
\hline Measure & Fish biomass \\
\hline Measure & Fish catch per unit effort \\
\hline Measure & Fish larval/egg abundance \\
\hline Measure & Fish migratory abundance \\
\hline Measure & Fish population size \\
\hline Measure & Fish species abundance \\
\hline Measure & Fish species presence-absence \\
\hline Measure & Fish species richness \\
\hline Measure & Host fish abundance \\
\hline Measure & Macroinvertebrate abundance \\
\hline Measure & Macroinvertebrate taxa abundance \\
\hline Measure & Migratory fish abundance \\
\hline Measure & Mussel population size \\
\hline Measure & Quarterly abundance counts of terrestrial \\
\hline species \\
\hline Measure & Vegetation biomass \\
\hline Statistic & Deer abundance \\
\hline Statistic & Fish abundance- max \\
\hline Statistic & Fish abundance - min \\
\hline Statistic & Fish catch per unit effort \\
\hline Statistic & Fish catch per unit effort - average \\
\hline Statistic & Fish population biomass \\
\hline Statistic & Fish population density \\
\hline Statistic & Fish population density age-class \\
\hline Statistic & Fish population size \\
\hline Statistic & Fish total catch \\
\hline Statistic & Fish YOY density \\
\hline Statistic & Goat abundance \\
\hline
\end{tabular}

\begin{tabular}{|l|l|}
\hline Metric Type & \multicolumn{1}{|c|}{ Metric Name } \\
\hline Statistic & Macroinvertebrate density \\
\hline Statistic & Macroinvertebrate drift density \\
\hline Statistic & Macrophyte abundance Domin-Krajina \\
\hline Statistic & Macrophyte coverage \\
\hline Statistic & Mussel population density \\
\hline Statistic & Non-native Mussel density \\
\hline Statistic & Non-native Mussel larval abundance \\
\hline Statistic & Phytoplankton abundance \\
\hline Statistic & Phytoplankton biomass \\
\hline Statistic & Tree abundance- basal area \\
\hline Statistic & Tree abundance - volume \\
\hline Statistic & Tree density \\
\hline Statistic & Vegetation abundance \\
\hline Indicator & Annual fish stock surveys \\
\hline Indicator & Change Phytoplankton abundance \\
\hline Indicator & Deviation fish population size \\
\hline Indicator & Fish \\
\hline Indicator & Macroinvertebrate sampling \\
\hline Indicator & Snail surveys \\
\hline
\end{tabular}

Frequencies based on EMH Catalog Contents as of January 31, 2017

ORNL Catalog of Environmental Metrics for Hydropower - 78 


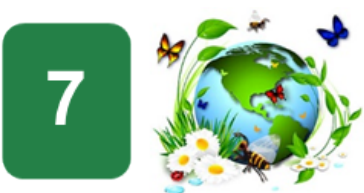

BB Biota and Biodiversity

\begin{tabular}{|c|c|c|}
\hline Sector & studies & metrics \\
\hline IHA & 4 & 0 \\
\hline LIHI & 5 & 0 \\
\hline FERC & 5 & 0 \\
\hline $\begin{array}{l}\text { Peer- } \\
\text { Reviewed }\end{array}$ & & \\
\hline Literature & 247 & 12 \\
\hline Totals & 261 & 12 \\
\hline
\end{tabular}

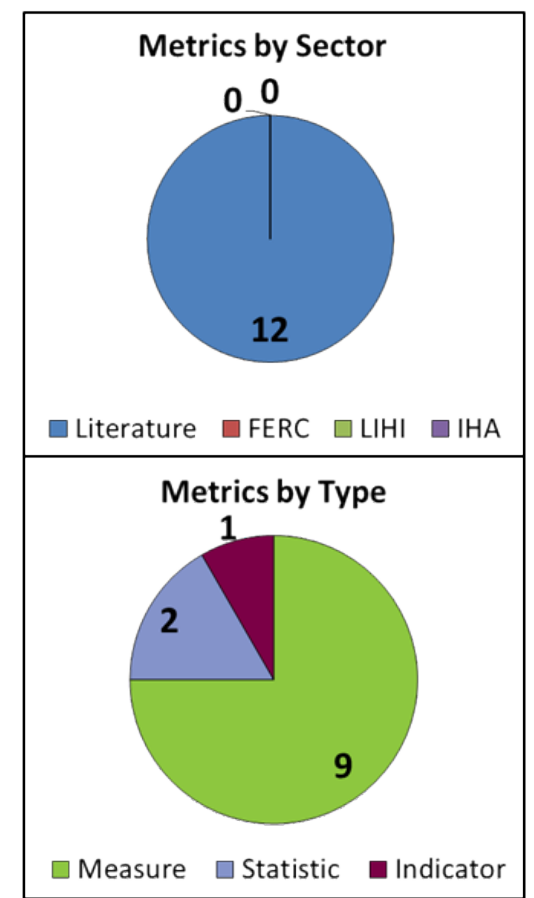

BB_Behavior, movement

Behavior of organisms, including movement pattern, distance, duration, timing, and frequency

\begin{tabular}{|l|l|}
\hline $\begin{array}{c}\text { Metric } \\
\text { Type }\end{array}$ & \multicolumn{1}{|c|}{ Metric Name } \\
\hline Measure & Fish movement \\
\hline Measure & Goat behavior 1 \\
\hline Measure & Goat daily movement \\
\hline Measure & Mussel movement \\
\hline Measure & Turtle movement \\
\hline Statistic & Mussel burial - mean \\
\hline Statistic & Mussel movement - mean \\
\hline Indicator & $\begin{array}{l}\text { Change fish life history } \\
\text { characteristic - spawn 2 }\end{array}$ \\
\hline
\end{tabular}

ORNL Catalog of Environmental Metrics for Hydropower - 79 


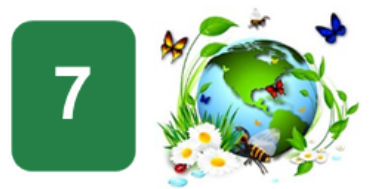

BB Biota and

Biodiversity

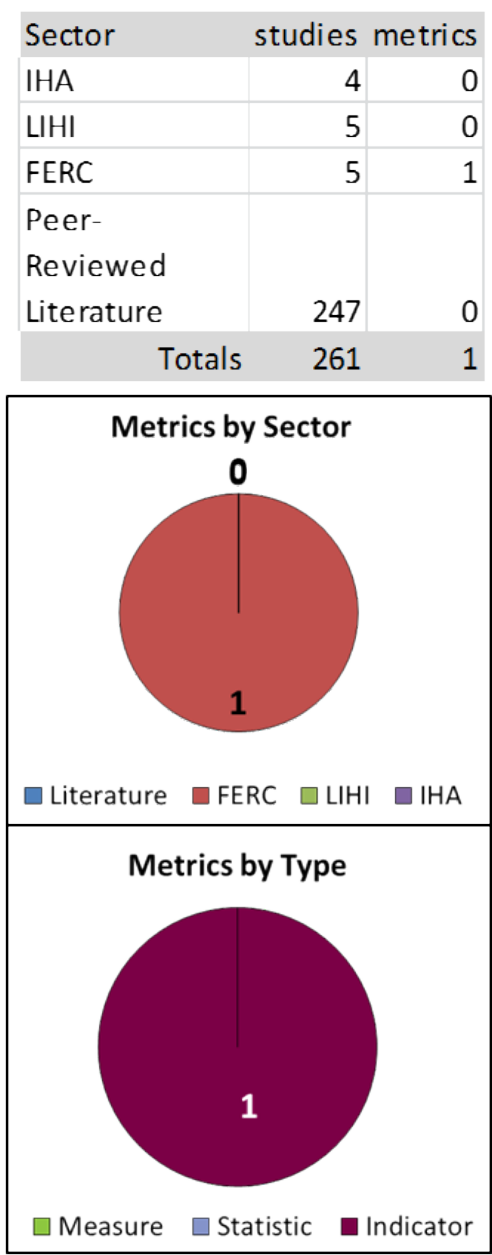

BB_Colonization,

extinction

\begin{tabular}{|c|c|}
\hline $\begin{array}{c}\text { Metric } \\
\text { Type }\end{array}$ & Metric Name \\
\hline Indicator & Macroinvertebrate colonization \\
\hline
\end{tabular}


3.7.4 BB_Demographics, age, sex, size

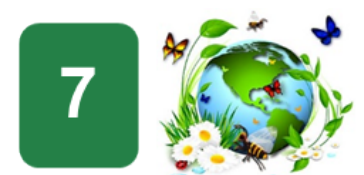

BB Biota and

Biodiversity

\begin{tabular}{|l|r|r|}
\hline Sector & studies & metrics \\
\hline IHA & 4 & 0 \\
\hline LIHI & 5 & 0 \\
\hline FERC & 5 & 0 \\
\hline \begin{tabular}{l|r|} 
Peer- \\
Reviewed \\
Literature
\end{tabular} & & \\
\hline \multicolumn{1}{|l|}{ Totals } & 247 & 35 \\
\hline
\end{tabular}

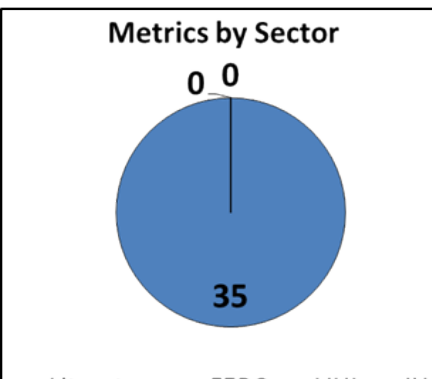

$\square$ Literature $\square$ FERC $\square$ LIHI $\square$ IHA

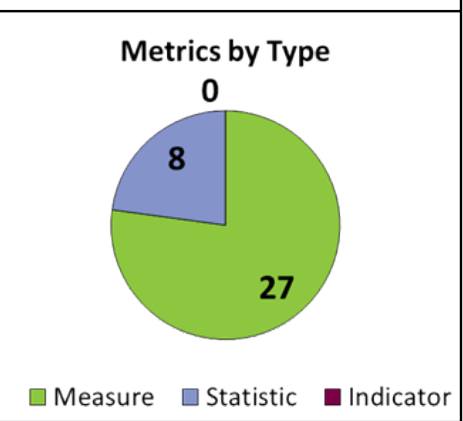

BB_Demographics, age, sex, size

\begin{tabular}{|l|l|}
\hline $\begin{array}{c}\text { Metric } \\
\text { Type }\end{array}$ & \multicolumn{1}{|c|}{ Metric Name } \\
\hline Measure & Fish age \\
\hline Measure & Fish length regulated site \\
\hline Measure & Fish length unregulated site \\
\hline Measure & Fish sex \\
\hline Measure & Fish size \\
\hline Measure & Goat age \\
\hline Measure & Goat sex \\
\hline Measure & Mussel age \\
\hline Measure & Mussel size \\
\hline Measure & Phytoplankton size \\
\hline Measure & Tree size \\
\hline Statistic & Fish size \\
\hline Statistic & Fish size - range \\
\hline Statistic & Fish size age-class \\
\hline Statistic & Mussel age - \% \\
\hline Statistic & Mussel size - \% \\
\hline
\end{tabular}




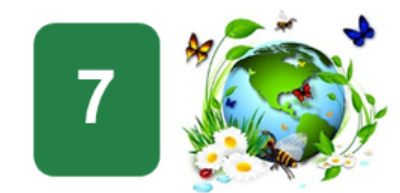

BB Biota and

Biodiversity

\begin{tabular}{|l|r|r|}
\hline Sector & studies & metrics \\
\hline IHA & 4 & 0 \\
\hline LIHI & 5 & 1 \\
\hline FERC & 5 & 9 \\
\hline Peer- & & \\
\hline Reviewed & & \\
Literature & 247 & 68 \\
\hline \multicolumn{1}{|r|}{ Totals } & 261 & 78 \\
\hline
\end{tabular}

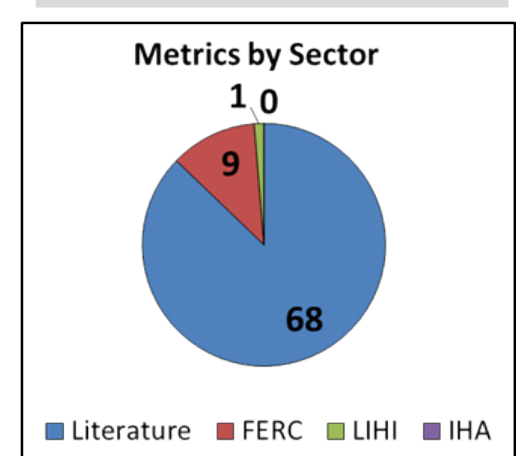

Metrics by Type

Metrics by Type

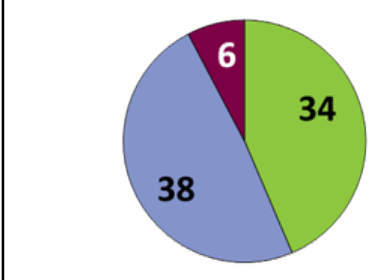

$\square$ Measure $\square$ Statistic
BB_Fitness, survival,

growth, condition,

reproduction, mortality

\begin{tabular}{|l|l|}
\hline \multicolumn{1}{|c|}{ Metric Type } & \multicolumn{1}{|c|}{ Metric Name } \\
\hline Measure & Fish condition - stomach content \\
\hline Measure & Fish contaminant As concentration \\
\hline Measure & Fish contaminant Cd concentration \\
\hline Measure & Fish contaminant Cu concentration \\
\hline Measure & Fish contaminant Fe concentration \\
\hline Measure & Fish contaminant Hg concentration \\
\hline Measure & Fish contaminant Mn concentration \\
\hline Measure & Fish contaminant Pb concentration \\
\hline Measure & Fish kills - presence-absence \\
\hline Measure & Fish mortality \\
\hline Measure & Fish survival - abundance \\
\hline Measure & Mussel larval abundance \\
\hline Measure & Non-native Mussel life history \\
characteristic 1
\end{tabular}

Fitness, survival, growth, condition, reproduction, or mortality of organisms

\begin{tabular}{|l|l|}
\hline \multicolumn{1}{|c|}{ Metric Type } & \multicolumn{1}{|c|}{ Metric Name } \\
\hline Statistic & Fish gonadosomatic index \\
\hline Statistic & Fish growth rate \\
\hline Statistic & Fish growth rate age-class mean \\
\hline Statistic & Fish Iarval/egg abundance \\
\hline Statistic & Fish mortality - \% \\
\hline Statistic & Fish mortality - average \\
\hline Statistic & Fish mortality - maximum \\
\hline Statistic & Fish mortality - minimum \\
\hline Statistic & Fish mortality rate age-class mean \\
\hline Statistic & Fish recruitment \\
\hline Statistic & Fish survival \\
\hline Statistic & Fish survival - \% \\
\hline Statistic & Fish turbine survival \\
\hline Statistic & $\begin{array}{l}\text { Maximum phytoplankton growth } \\
\text { rate }\end{array}$ \\
\hline Statistic & Mussel growth rate \\
\hline Statistic & Mussel mortality - \% \\
\hline Statistic & Mussels stranded - \% \\
\hline Statistic & Percent mortality \\
\hline Indicator & Fish condition age-class Fulton \\
\hline Indicator & Fish gonadosomatic index \\
\hline Indicator & Fish spawning/recruitment \\
\hline Indicator & Fish YoY survival \\
\hline Indicator & Mussel Larval Dispersal Indicator \\
\hline Indicator & Mussel Reproduction Indicator \\
\hline & \\
\hline
\end{tabular}

ORNL Catalog of Environmental Metrics for Hydropower - 82 


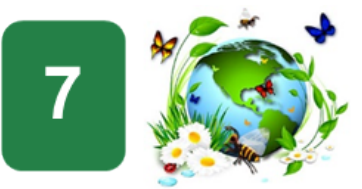

BB Biota and

Biodiversity

\begin{tabular}{|l|r|r|}
\hline Sector & studies & metrics \\
\hline IHA & 4 & 0 \\
\hline LIHI & 5 & 0 \\
\hline FERC & 5 & 30 \\
\hline \begin{tabular}{l|r|} 
Peer- \\
Reviewed \\
Literature
\end{tabular} & 247 & 36 \\
\hline & & \\
\hline Totals & 261 & 66 \\
\hline
\end{tabular}

Metrics by Sector

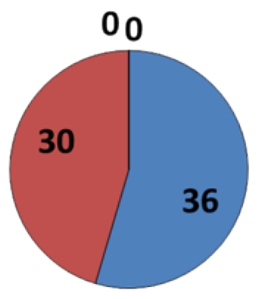

- Literature $\square$ FERC $\square$ LIHI $\square$ IHA

\section{Metrics by Type}

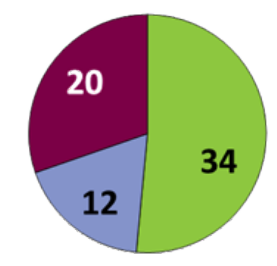

$\square$ Measure $\square$ Statistic

\section{BB_Functional group, or}

species or trait

composition

\begin{tabular}{|l|l|}
\hline Metric Type & \multicolumn{1}{|c|}{ Metric Name } \\
\hline Measure & Amphibian Species Composition \\
\hline Measure & Aquatic Mammal Species Composition \\
\hline Measure & Aquatic Reptile Species Composition \\
\hline Measure & Bird Wildlife Species Composition \\
\hline Measure & Butterfly Species Composition \\
\hline Measure & $\begin{array}{l}\text { Fish community composition - broad cast } \\
\text { spawning }\end{array}$ \\
\hline Measure & $\begin{array}{l}\text { Fish community composition - complex } \\
\text { spawning }\end{array}$ \\
\hline Measure & $\begin{array}{l}\text { Fish community composition - Cruiser } \\
\text { morphology }\end{array}$ \\
\hline Measure & $\begin{array}{l}\text { Fish community composition - Feeding guilds } \\
\text { (Herbivore/Detritivore) }\end{array}$ \\
\hline Measure & $\begin{array}{l}\text { Fish community composition - Feeding guilds } \\
\text { (Insectivore) }\end{array}$ \\
\hline Measure & $\begin{array}{l}\text { Fish community composition - Hugger } \\
\text { morphology }\end{array}$ \\
\hline Measure & $\begin{array}{l}\text { Fish Community Composition - life history } \\
\text { strategies }\end{array}$ \\
\hline Measure & Fish Community Composition - nest type \\
\hline Measure & Fish community composition - Pool habitat \\
\hline Measure & $\begin{array}{l}\text { Fish community composition - Slackwater } \\
\text { habitat }\end{array}$ \\
\hline Measure & Fish community species composition \\
\hline Measure & $\begin{array}{l}\text { Macroinvertebrate functional feeding group } \\
\text { abundance }\end{array}$ \\
\hline Measure & Riparian Vegetation Composition \\
\hline Measure & Riparian Wildlife Composition \\
\hline Measure & Terrestrial Mammal Wildlife Composition \\
\hline Measure & Uplant Fishery - Presence-absence \\
\hline Measure & Vegetation community species composition \\
\hline Measure & Vegetation type \\
\hline
\end{tabular}

Grouping of organisms by functional or trait status, percentage composition

\begin{tabular}{|l|l|}
\hline Metric Type & \multicolumn{1}{|c|}{ Metric Name } \\
\hline Measure & Wetland Vegetation Compostion \\
\hline Statistic & $\begin{array}{l}\text { Change in fish community composition - } \\
\text { functional type }\end{array}$ \\
\hline Statistic & Fish Community Composition - \% \\
\hline Statistic & Fish Community Composition - historical \\
\hline Statistic & Fish Species Composition - \% \\
\hline Statistic & $\begin{array}{l}\text { Macroinvertebrate community composition - \% } \\
\text { taxa }\end{array}$ \\
\hline Statistic & Macroinvertebrate species richness \\
\hline Statistic & Natural vegetation as semblage change \\
\hline Statistic & Natural vegetation assemblage succession \\
\hline Statistic & Recreational fishing effort - \% of time \\
\hline Statistic & Recreational fishing harvest - \% of catch \\
\hline Indicator & Canadian Ecological Flow Generalist Index \\
\hline Indicator & Canadian Ecological Flow Specialist Index \\
\hline Indicator & $\begin{array}{l}\text { Change in fish community composition - } \\
\text { equilibrium life histories }\end{array}$ \\
\hline Indicator & $\begin{array}{l}\text { Change in fish community composition - } \\
\text { opportunistic life his tories }\end{array}$ \\
\hline Indicator & $\begin{array}{l}\text { Change in fish community composition - } \\
\text { periodic life histories }\end{array}$ \\
\hline Indicator & Colwater Fisheries - Pres ence-absence \\
\hline Indicator & Coolwater Fish Community Indicator \\
\hline Indicator & Fish Community Composition - \% \\
\hline Indicator & Fish Community Health \\
\hline Indicator & Fish Taxonomic Distinctiveness Index \\
\hline Indicator & Fis hery Resources Indicator \\
\hline Indicator & Macroinvertebrate Community Health \\
\hline Indicator & Riparian vegetation alteration \\
\hline Indicator & Sportfishing Satisfaction Rating \\
\hline Indicator & $\begin{array}{l}\text { Tree Jaccard's coefficient of community } \\
\text { similarity }\end{array}$ \\
\hline Indicator & Tree Sorensen's Similarity Index \\
\hline Indicator & Warmwater Fish Community Indicator \\
\hline & Fren EM \\
\hline
\end{tabular}

Frequencies based on EMH Catalog Contents as of January 31, 2017

ORNL Catalog of Environmental Metrics for Hydropower - 83 


\subsubsection{BB_Functional group, or species or trait composition}

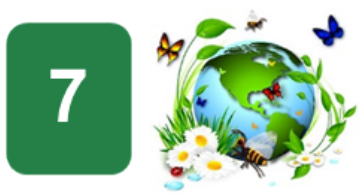

BB Biota and

Biodiversity

\begin{tabular}{|c|c|c|}
\hline Sector & studies & metrics \\
\hline IHA & 4 & 0 \\
\hline LIHI & 5 & 0 \\
\hline FERC & 5 & 30 \\
\hline $\begin{array}{l}\text { Peer- } \\
\text { Reviewed }\end{array}$ & & \\
\hline Literature & 247 & 36 \\
\hline Totals & 261 & 66 \\
\hline
\end{tabular}

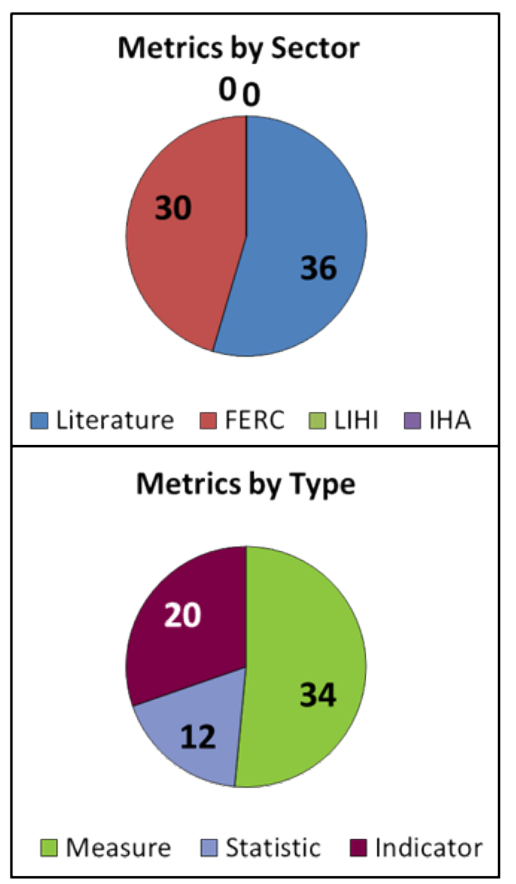

\section{BB_Functional group, or}

species or trait

composition

\begin{tabular}{|l|l|}
\hline Metric Type & \multicolumn{1}{|c|}{ Metric Name } \\
\hline Measure & Amphibian Species Composition \\
\hline Measure & Aquatic Mammal Species Composition \\
\hline Measure & Aquatic Reptile Species Composition \\
\hline Measure & Bird Wildlife Species Composition \\
\hline Measure & Butterfly Species Composition \\
\hline Measure & $\begin{array}{l}\text { Fish community composition - broad cast } \\
\text { spawning }\end{array}$ \\
\hline Measure & $\begin{array}{l}\text { Fish community composition - complex } \\
\text { spawning }\end{array}$ \\
\hline Measure & $\begin{array}{l}\text { Fish community composition - Cruiser } \\
\text { morphology }\end{array}$ \\
\hline Measure & $\begin{array}{l}\text { Fish community composition - Feeding guilds } \\
\text { (Herbivore/Detritivore) }\end{array}$ \\
\hline Measure & $\begin{array}{l}\text { Fish community composition - Feeding guilds } \\
\text { (Insectivore) }\end{array}$ \\
\hline Measure & $\begin{array}{l}\text { Fish community composition - Hugger } \\
\text { morphology }\end{array}$ \\
\hline Measure & $\begin{array}{l}\text { Fish Community Composition - life his tory } \\
\text { strategies }\end{array}$ \\
\hline Measure & Fish Community Composition - nest type \\
\hline Measure & Fish community composition - Pool habitat \\
\hline Measure & $\begin{array}{l}\text { Fish community composition - Slackwater } \\
\text { habitat }\end{array}$ \\
\hline Measure & Fish community species composition \\
\hline Measure & $\begin{array}{l}\text { Macroinvertebrate functional feeding group } \\
\text { abundance }\end{array}$ \\
\hline Measure & Riparian Vegetation Composition \\
\hline Measure & Riparian Wildlife Composition \\
\hline Measure & Terrestrial Mammal Wildlife Composition \\
\hline Measure & Trout Fishery - Presence-absence \\
\hline Measure & Upland Vegetation Composition \\
\hline Measure & Vegetation community species composition \\
\hline Measure & Vegetation type \\
\hline
\end{tabular}

Grouping of organisms by functional or trait status, percentage composition

\begin{tabular}{|l|l|}
\hline Metric Type & \multicolumn{1}{|c|}{ Metric Name } \\
\hline Measure & Wetland Vegetation Compostion \\
\hline Statistic & $\begin{array}{l}\text { Change in fish community composition - } \\
\text { functional type }\end{array}$ \\
\hline Statistic & Fish Community Composition - \% \\
\hline Statistic & Fish Community Composition - historical \\
\hline Statistic & Fish Species Composition - \% \\
\hline Statistic & $\begin{array}{l}\text { Macroinvertebrate community composition - \% } \\
\text { taxa }\end{array}$ \\
\hline Statistic & Macroinvertebrate species richnes s \\
\hline Statistic & Natural vegetation assemblage change \\
\hline Statistic & Natural vegetation as semblage succession \\
\hline Statistic & Recreational fishing effort - \% of time \\
\hline Statistic & Recreational fishing harvest - \% of catch \\
\hline Indicator & Canadian Ecological Flow Generalist Index \\
\hline Indicator & Canadian Ecological Flow Specialist Index \\
\hline Indicator & $\begin{array}{l}\text { Change in fish community composition - } \\
\text { equilibrium life histories }\end{array}$ \\
\hline Indicator & $\begin{array}{l}\text { Change in fish community composition - } \\
\text { opportunistic life histories }\end{array}$ \\
\hline Indicator & $\begin{array}{l}\text { Change in fish community composition - } \\
\text { periodic life histories }\end{array}$ \\
\hline Indicator & Colwater Fisheries - Pres ence-absence \\
\hline Indicator & Coolwater Fish Community Indicator \\
\hline Indicator & Fish Community Composition - \% \\
\hline Indicator & Fish Community Health \\
\hline Indicator & Fish Taxonomic Dis tinctiveness Index \\
\hline Indicator & Fishery Resources Indicator \\
\hline Indicator & Macroinvertebrate Community Health \\
\hline Indicator & Riparian vegetation alteration \\
\hline Indicator & Sportfishing Satisfaction Rating \\
\hline Indicator & $\begin{array}{l}\text { Tree Jaccard's coefficient of community } \\
\text { similarity }\end{array}$ \\
\hline Indicator & Tree Sorens en's Similarity Index \\
\hline Indicator & Warmwater Fish Community Indicator \\
\hline
\end{tabular}

Frequencies based on EMH Catalog Contents as of January 31, 2017

ORNL Catalog of Environmental Metrics for Hydropower - 84 


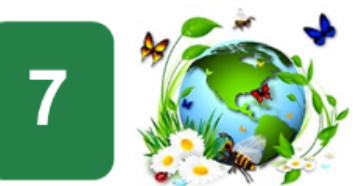

BB Biota and Biodiversity

\begin{tabular}{|l|r|r|}
\hline Sector & studies & metrics \\
\hline IHA & 4 & 1 \\
\hline LIHI & 5 & 1 \\
\hline FERC & 5 & 1 \\
\hline \begin{tabular}{l|r} 
Peer- \\
Reviewed \\
Literature
\end{tabular} & & \\
\hline \multicolumn{1}{|r|}{ Totals } & 247 & 9 \\
\hline
\end{tabular}

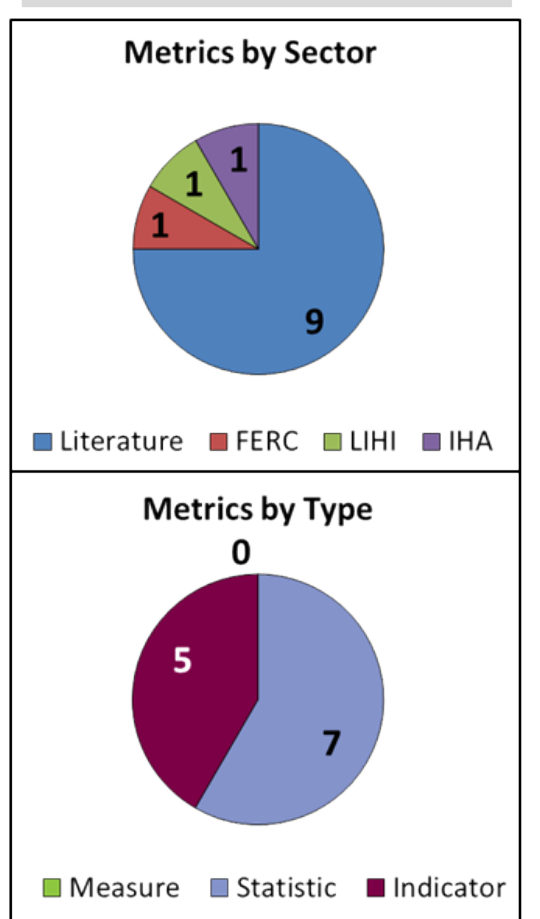

BB_Genetics, mixing,

metapopulation
Genetics and population mixing, including metapopulation dynamics

\begin{tabular}{|l|l|}
\hline \multicolumn{1}{|c|}{$\begin{array}{c}\text { Metric } \\
\text { Type }\end{array}$} & \multicolumn{1}{c|}{ Metric Name } \\
\hline Statistic & Genetic diversity - allelic richness \\
\hline Statistic & Genetic diversity - heterozygosity \\
\hline Statistic & Genetic diversity - inbreeding coeff \\
\hline Statistic & Genetic diversity - population structure \\
\hline Statistic & Proportion polymorphic loci \\
\hline Indicator & Gene flow \\
\hline Indicator & Genetic population mixing \\
\hline Indicator & $\begin{array}{l}\text { Morphological and genetic analyses of } \\
\text { armored catfish }\end{array}$ \\
\hline Indicator & Nei's genetic diversity \\
\hline
\end{tabular}




\subsubsection{BB_Habitat, critical habitat, or surrogates of such}

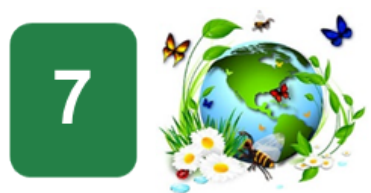

BB Biota and

Biodiversity

\begin{tabular}{|c|c|c|}
\hline Sector & studies & metrics \\
\hline IHA & 4 & 2 \\
\hline LIHI & 5 & 3 \\
\hline FERC & 5 & 35 \\
\hline $\begin{array}{l}\text { Peer- } \\
\text { Reviewed }\end{array}$ & & \\
\hline Literature & 247 & 17 \\
\hline Totals & 261 & 57 \\
\hline
\end{tabular}

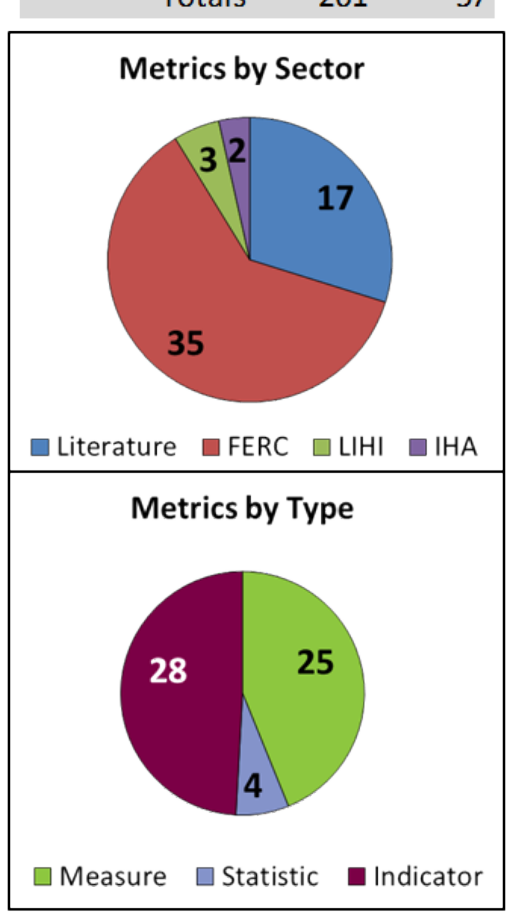

BB_Habitat, critical

Indices of habitat, area, suitability, and so on, for organisms habitat, or surrogates of

such

\begin{tabular}{|l|l|}
\hline Metric Type & \multicolumn{1}{|c|}{ Metric Name } \\
\hline Measure & Avian species nests/roosts \\
\hline Measure & Critical Habitat - length \\
\hline Measure & Critical Habitat - presence-abs ence \\
\hline Measure & EFH - pres ence-absence \\
\hline Measure & Emergent wetland area \\
\hline Measure & Essential Fish Habitat - pres ence-absence \\
\hline Measure & Fish distribution - area \\
\hline Measure & Fish habitat characteristic 1 - cover \\
\hline Measure & Fish spawning gravel volume \\
\hline Measure & Goat habitat characteristic 1 \\
\hline Measure & Goat habitat characteristic 2 \\
\hline Measure & Plant habitat characteristics 1 \\
\hline Measure & Reservoir water depth \\
\hline Measure & Shoreline habitat area \\
\hline Measure & Stream mesohabitat area \\
\hline Measure & Stream vegetation patch size \\
\hline Measure & $\begin{array}{l}\text { Upstream Diadromous Fish Habitat - presence- } \\
\text { absence }\end{array}$ \\
\hline Measure & Wetlant Habitat Type - categorical \\
\hline Statistic & Inundated mesohabitat area - \% \\
\hline Statistic & Relations hip fish density/ habitat type regulated \\
site
\end{tabular}

\begin{tabular}{|l|l|}
\hline Metric Type & \multicolumn{1}{|c|}{ Metric Name } \\
\hline Indicator & Deviation wetted habitat perimeter \\
\hline Indicator & Fish spawning habitat Indicator \\
\hline Indicator & Fish spawning habitat quality indicator \\
\hline Indicator & Habitat Quality Indicator \\
\hline Indicator & Habitat Suitability Index \\
\hline Indicator & Hydrology-fish as sociation correlation \\
\hline Indicator & Instream habitat alteration \\
\hline Indicator & Mussel habitat quality indicator \\
\hline Indicator & RBA score \\
\hline Indicator & Riparian habitat quality index \\
\hline Indicator & Riparian Habitat Quality Indicator \\
\hline Indicator & Stream Habitat Quality Indicator \\
\hline Indicator & Stream vegetation habitat characteristic \\
\hline Indicator & Terrestrial Wildlife Habitat Quality Indicator \\
\hline Indicator & Weighted Useable Area \\
\hline Indicator & Wetted habitat perimeter \\
\hline Indicator & Wetted habitat perimeter breakpoint \\
\hline Indicator & Years since construction \\
\hline
\end{tabular}

Frequencies based on EMH Catalog Contents as of January 31, 2017

ORNL Catalog of Environmental Metrics for Hydropower - 86 
3.7.10 BB_Internal composition nutrient abnormalities

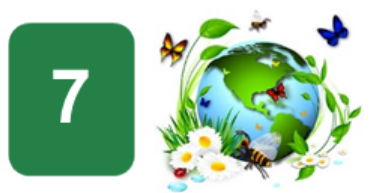

BB Biota and

Biodiversity

\begin{tabular}{|l|r|r|}
\hline Sector & studies metrics \\
\hline IHA & 4 & 0 \\
\hline LIHI & 5 & 0 \\
\hline FERC & 5 & 0 \\
\hline
\end{tabular}

Peer-

Reviewed

$\begin{array}{crr}\text { Literature } & 247 & 3 \\ \text { Totals } & 261 & 3\end{array}$

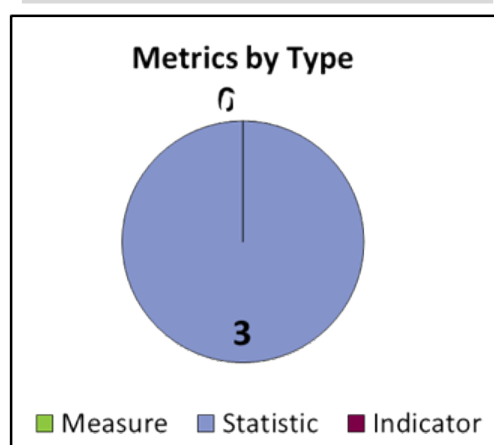

$\square$ Measure $\square$ Statistic

Metrics by Sector

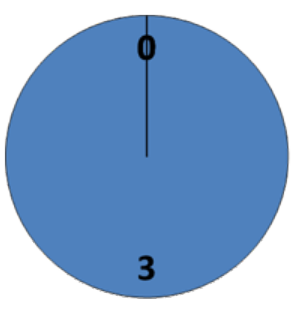

Literature $\square$ FERC $\square$ LIHI $\square$ IHA
BB_Internal composition Nutritional composition and makeup of organisms, including nutrient abnormalities

elemental stoichiometry. It includes levels of internal homeostasis, as well as morphological, genetic, or hormonal abnormalities caused by contaminants

\begin{tabular}{|l|l|}
\hline Metric Type & \multicolumn{1}{|c|}{ Metric Name } \\
\hline Statistic & Nitrogen-to-carbon ratio in phytoplankton \\
\hline Statistic & Oxygen-to-carbon ratio in phytoplankton \\
\hline Statistic & Phosphorous-to-carbon ratio in phytoplankton \\
\hline
\end{tabular}


3.7.11 BB_Internal composition nutrient abnormalities

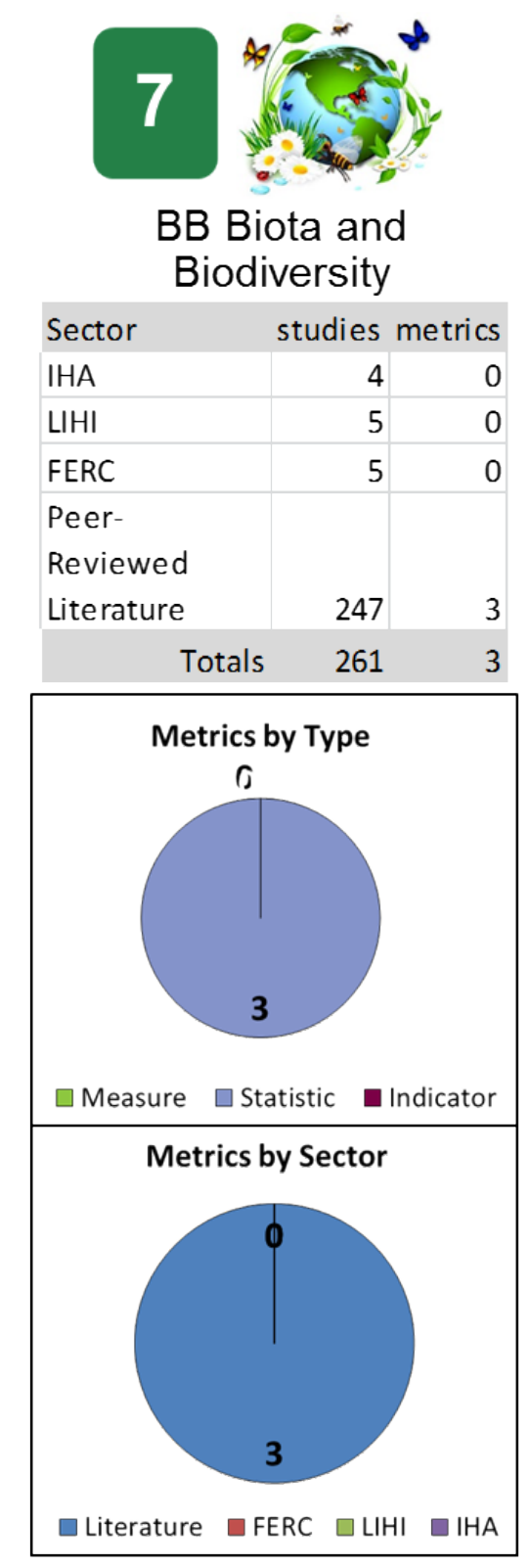
BB_Internal composition nutrient abnormalities
Nutritional composition and makeup of organisms, including elemental stoichiometry. It includes levels of internal homeostasis, as well as morphological, genetic, or hormonal abnormalities caused by contaminants

\begin{tabular}{|l|l|}
\hline Metric Type & \multicolumn{1}{|c|}{ Metric Name } \\
\hline Statistic & Nitrogen-to-carbon ratio in phytoplankton \\
\hline Statistic & Oxygen-to-carbon ratio in phytoplankton \\
\hline Statistic & Phosphorous-to-carbon ratio in phytoplankton \\
\hline
\end{tabular}

\begin{tabular}{|c|c|}
\hline Reviewed & \\
\hline Literature & 247 \\
\hline Totals & 261 \\
\hline
\end{tabular}




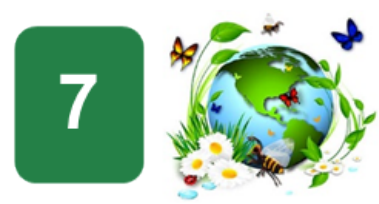

BB Biota and Biodiversity

\begin{tabular}{|c|c|c|}
\hline \multicolumn{3}{|c|}{ - } \\
\hline Sector & studies & metrics \\
\hline IHA & 4 & 0 \\
\hline LIHI & 5 & 0 \\
\hline FERC & 5 & 7 \\
\hline $\begin{array}{l}\text { Peer- } \\
\text { Reviewed }\end{array}$ & & \\
\hline Literature & 247 & 16 \\
\hline Totals & 261 & 23 \\
\hline
\end{tabular}
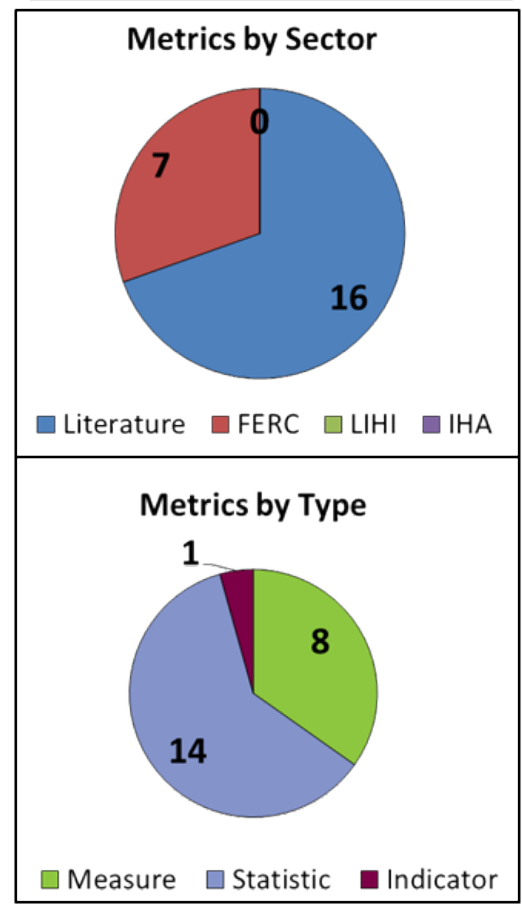

$\square$ Measure $\square$ Statistic $\square$ Indicator

\section{BB_Life history trait \\ characteristics}

\begin{tabular}{|l|l|}
\hline $\begin{array}{c}\text { Metric } \\
\text { Type }\end{array}$ & \multicolumn{1}{|c|}{ Metric Name } \\
\hline Measure & Fish life history characteristic - spawn 2 \\
\hline Measure & Fish morphology characteristic 1 \\
\hline Measure & Mussel life history characteristic 1 \\
\hline Measure & Non-native Mussel life history characteristic 2 \\
\hline Measure & Non-native Mussel life history characteristic 3 \\
\hline Statistic & Date downstream migration \\
\hline Statistic & Date upstream migration \\
\hline Statistic & Duration downstream migration \\
\hline Statistic & Duration upstream migration \\
\hline Statistic & Fish life history characteristic - early development (incubation rate) \\
\hline Statistic & Fish life history characteristic - spawn 3 \\
\hline Statistic & Fish life history characteristic - spawn 4 \\
\hline Statistic & Fish life history characteristic - spawn duration \\
\hline Statistic & Fish morphology characteristic 2 \\
\hline Indicator & Mussel-Host Fish Strength Indicator \\
\hline
\end{tabular}

Life history trait characteristics and their values, such as duration of spawning, fecundity, reproductive mode (note that this category deals only with characteristics themselves and not the composition of the community. See Functional group, or species or trait composition) 


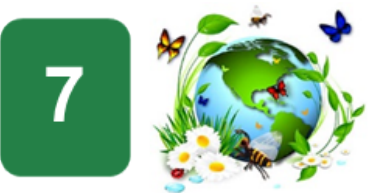

BB Biota and Biodiversity

\begin{tabular}{|l|r|r|}
\hline Sector & studies metrics \\
\hline IHA & 4 & 13 \\
\hline LIHI & 5 & 19 \\
\hline FERC & 5 & 22 \\
\hline
\end{tabular}

Peer-

Reviewed

\begin{tabular}{|c|l|l|}
\hline Literature & 247 & 12 \\
\hline Totals & 261 & 66 \\
\hline
\end{tabular}

\begin{tabular}{|l|}
\hline \\
\hline 261
\end{tabular}
BB_Presence, absence, occupancy, or detection
Organism presence/absence in an area (including pseudo-absence), occupancy, and detection probability

\begin{tabular}{|c|c|}
\hline Metric Type & Metric Name \\
\hline Measure & Coldwater Fish - presence-absence \\
\hline Measure & $\begin{array}{l}\text { Diadromous fish - historical presence- } \\
\text { absence }\end{array}$ \\
\hline Measure & Diadromous fish - presence-absence \\
\hline Measure & $\begin{array}{l}\text { Federally listed species - pre sence- } \\
\text { absence }\end{array}$ \\
\hline Me asure & $\begin{array}{l}\text { Fe de rally listed species of concern - } \\
\text { presence-absence }\end{array}$ \\
\hline Measure & Fish detection \\
\hline Measure & Fish range \\
\hline Measure & Fish species presence-absence \\
\hline Measure & Goat distribution \\
\hline Measure & Goat range \\
\hline Measure & IEPP - presence-absence \\
\hline Measure & Invasive macrophyte presence/absence \\
\hline Measure & Native macrophyte presence/absence \\
\hline Measure & Non-native fish - presence-absence \\
\hline Measure & Non-native vegetation - presence-absence \\
\hline Measure & Nusiance macrophyte presence-absence \\
\hline Measure & Phytoplankton species presence-absence \\
\hline Measure & $\begin{array}{l}\text { River/Potadromous fish - presence- } \\
\text { absence }\end{array}$ \\
\hline Me asure & Sensitive species - presence-absence \\
\hline Measure & $\begin{array}{l}\text { Sensitive vegetation species - presence- } \\
\text { absence }\end{array}$ \\
\hline Measure & Vegetation flowering - presence \\
\hline Measure & Wetland Habitat - presence-absence \\
\hline
\end{tabular}

\begin{tabular}{|l|l|}
\hline Metric Type & \multicolumn{1}{|c|}{ Metric Name } \\
\hline Statistic & Deer detection \\
\hline Statistic & Deer distribution - patch occupancy \\
\hline Indicator & Baseline aquatic flora and fauna \\
\hline Indicator & $\begin{array}{l}\text { Baseline survey of potentially impacted } \\
\text { species }\end{array}$ \\
\hline Indicator & Baseline terrestrial wildlife \\
\hline Indicator & Identification of target species \\
\hline Indicator & $\begin{array}{l}\text { Identificaton of endangered, vulnerable \& } \\
\text { rare species }\end{array}$ \\
\hline Indicator & Monitoring of biodiversity impacts \\
\hline Indicator & Monitoring of biological trends \\
\hline Indicator & Quarterly survey of terrestrial species \\
\hline Indicator & $\begin{array}{l}\text { Quarterly surveys of plankton, benthos } \\
\text { and fish }\end{array}$ \\
\hline Indicator & $\begin{array}{l}\text { Rapid assessment of cumulative } \\
\text { biodiversity impacts }\end{array}$ \\
\hline Indicator & Special survey of key fish species \\
\hline Indicator & Special surveys of key terrestrial species \\
\hline Indicator & Transmission line impacts on biodiversity \\
\hline
\end{tabular}

ORNL Catalog of Environmental Metrics for Hydropower - 90 
3.7.14 BB_Richness, diversity, evenness, or IBI types of measures

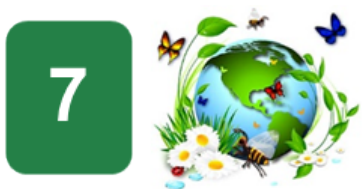

BB Biota and

Biodiversity

\begin{tabular}{|c|c|c|}
\hline Sector & studies & metrics \\
\hline IHA & 4 & 0 \\
\hline LIHI & 5 & 0 \\
\hline FERC & 5 & 21 \\
\hline $\begin{array}{l}\text { Peer- } \\
\text { Reviewed }\end{array}$ & & \\
\hline Literature & 247 & 35 \\
\hline Totals & 261 & 56 \\
\hline
\end{tabular}
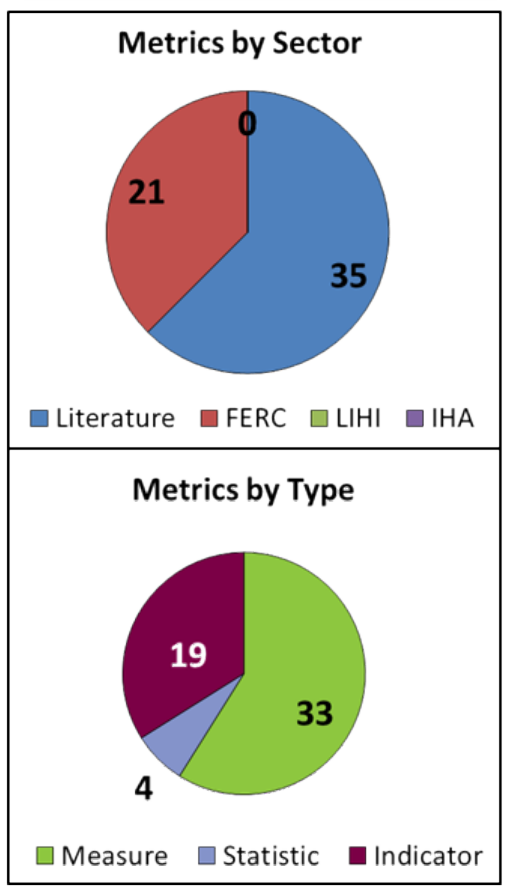

BB_Richness, diversity,

evenness, or IBI types of

measures

\begin{tabular}{|l|l|}
\hline Metric Type & \multicolumn{1}{|c|}{ Metric Name } \\
\hline Me asure & Amphibian Species Richness \\
\hline Measure & Aquatic Mammal Species Richness \\
\hline Me asure & Aquatic Reptile Species Richness \\
\hline Me asure & Bird Species Richness \\
\hline Me asure & Bufferly Species Richness \\
\hline Me asure & Fish species richness \\
\hline Measure & Fish Species Richness - historical \\
\hline Measure & IEPP - richness \\
\hline Me asure & Macroinvertebrate species richness \\
\hline Measure & Macrophyte species richness \\
\hline Measure & Mussel species richness \\
\hline Measure & Phytoplankton species richness \\
\hline Measure & Terrestrial Mammal Wildlife Species \\
& Mammal Richness \\
\hline Me asure & Warmwater Fish Species Richness \\
\hline Measure & Wetland Vegetation Species Richness \\
\hline Statistic & Fish species density \\
\hline Statistic & Fish species richness \\
\hline Statistic & Invasive macrophyte species richness \\
\hline Statistic & Native macrophyte species richness \\
\hline Indicator & Aquatic Resources Quality Indicator \\
\hline Indicator & Diversity in the Environmental Indicator \\
\hline Indicator & Ecological Benefit Indicator \\
\hline Indicator & Ecological condition \\
\hline Indicator & Macroinvertebrate Community Health \\
\hline Indicator & Macroinvertebrate Pielou evenness \\
\hline Indicator & Macroinverte brate Shannon-Weiner \\
diversity
\end{tabular}

Species richness, diversity, evenness, or indices-of-biotic-integrity metrics used to characterize one or more components of the biotic community

\begin{tabular}{|l|l|}
\hline Metric Type & \multicolumn{1}{|c|}{ Metric Name } \\
\hline Indicator & Phytoplankton Shannon-Wiener index \\
\hline Indicator & Terrestrial Resources Quality Indicator \\
\hline Indicator & Tree Berger-Parker Dominance Index \\
\hline Indicator & Tree Beta diversity \\
\hline Indicator & Tree Shannon-Weiner Diversity Index \\
\hline Indicator & Tree Simpson's Evenness index \\
\hline Indicator & Tree Simpson's Reciprocal Index \\
\hline Indicator & Tree Species Dominance Index \\
\hline
\end{tabular}

ORNL Catalog of Environmental Metrics for Hydropower - 91 


\section{REFERENCES:}

Hydropower Sustainability Assessment Protocol. November 2010 "Hydropower Sustainability Assessment Protocol"(220 page report) available at http://www.hydrosustainability.org/getattachment/7e212656-9d26-4ebc-96b8-1f27eaebc2ed/The-Hydropower-Sustainability-Assessment-Protocol.aspx

M.J. Sale, D. Hall, and J. Keil, 2016. Low Impact Hydropower Institute Certification Handbook, $2^{\text {nd }}$ Edition. Low Impact Hydropower Institute, Harrington Park, NJ.

Pracheil, Brenda M., Ryan A. McManamay, Esther S. Parish, Shelaine L. Curd, Brennan T. Smith. June 2016. Environmental Metrics for New Hydropower: Terminology and Categories ORNL TM-2016/720.

Schramm, M. P, M. S Bevelhimer, and C. R DeRolph. 2016. A Synthesis Of Environmental And Recreational Mitigation Requirements At Hydropower Projects In The United States. Environmental Science And Policy: 87-96. 


\title{
APPENDIXA. SYSTEMATIC REVIEW OF PEER-REVIEWED SCHOLARLY LITERATURE ON HYDROPOWER METRICS
}

\author{
Systematic review of peer-reviewed scholarly literature on hydropower metrics
}

\section{Generation of search strings}

This study employs a systematic review of scientific peer-review journal articles to summarize metrics that have been used to quantify the effects of hydropower. Systematic reviews set forth rigorous and repeatable study inclusion criteria and documentation (including stakeholder input, where appropriate) of search terms, search dates, and which studies were and were not included so that the body of literature discovered and included in the review is not only replicable, but is also unbiased with respect to point-ofview (CEE 2013). For example, one hydropower stakeholder conducting a systematic review may only choose a search string such as "hydropower impact" which suggests negative environmental consequences, whereas a different hydropower stakeholder may choose the search string "hydropower effect" which is impartial to the direction of change. While each of these search strings will likely discover some overlapping literature, it is also likely that there will be some peer-reviewed articles that appear in one search string and not the other. In this example, both search strings would be important to include because all stakeholder viewpoints are imperative for presenting an unbiased review and using search strings that discover all relevant bodies of literature are important. Therefore, obtaining stakeholder input on search strings is a critical component for obtaining an unbiased perspective of the literature.

Whenever possible, open-access or open-access compatible databases and tools were used to ensure transparency and availability to the entire stakeholder community with the understanding that many will not have access to proprietary search and reference management tools.

Also, although the importance and relevance of gray literature to hydropower regulatory decision making is recognized, it was excluded from the systematic review for three primary reasons. First, representing this body of literature in a consistent or representative way is difficult due to difficulties in securing and discovering texts. Second, reviewing gray literature presents a unique challenge compared to peer-review literature because peer-reviewed papers are, by definition, reviewed by other experts. Evaluation of the scientific rigor of gray literature can vary by the author/ authoring organization's policies and encompasses the spectrum from an equally or more rigorous process to that found in the peer-reindicview literature to no additional review. Third, a parallel review of hydropower regulation and certification documents will be ongoing as part of this project that will incorporate gray literature that has been used in hydropower decision-making. Furthermore, because gray literature included in hydropower regulation and certification documents has been scrutinized as part of the licensing or certification process, there is presumably some threshold of scientific rigor that has been surpassed.

Initial search strings (insert table of search strings) were obtained from discussion among four ORNL research staff with hydropower industry, hydropower environmental, and sustainability science expertise.

The intent of these discussions was to devise a list of search strings for the systematic review that would represent multiple stakeholder viewpoints and generate a comprehensive list of literature search results that was not overly duplicative, but still left as few gaps in overall search results as possible. These discussions resulted in the creation of 216 unique search strings containing one environmental term (e.g., "Land cover", biodiversity) and one hydropower term (e.g., dam, powerhouse). The "scientific categories" from the "Hydropower Environmental Terminology" paper produced as part of this project formed the basis for the environmental terms in addition to major biological groups and terms that ORNL staff thought would help yield unique and pertinent search results. Hydropower terms in search strings were named based on input from ORNL hydropower engineering staff. The Boolean term "OR" was used 
in cases where multiple forms of words searching for the same thing were used in search strings (e.g., "Hydropower OR Hydroelectric") and asterisks were used to enable searching for multiple endings on words (e.g., "Fragment*" would search for "fragment, fragmented, fragments, fragmentation, fragmenting, etc.). Because some of the search strings were quite general and yielded large amounts of information that did not pertain to hydropower or environmental sciences, an initial screening of search strings was conducted using Google Scholar searches for each strings recording the number of results (CEE 2013). The titles of the first seven results (the number of search results that fit on the average screen) were then surveyed as an indicator of whether the string would provide useful results for this review by noting whether the results contained environmental information. If any one of the first seven results pertained to environmental effects in the title, the search string was categorized as containing information potentially useful for this project and was retained for further use. If search strings did not contain information potentially useful for this project, the search string was discarded. For instance, the search string "metric* dam*" (see Table 1 caption for explanation of asterisks and quotations in search strings) was discarded because all seven results were related to the field of particle physics. In total, 150 unique search strings were retained from the initial search screen. It is this list of 150 search strings that will be presented to stakeholders for input.

\section{Study inclusion criteria}

The final list of search strings was searched using Google Scholar during 9 September, 2016 through 22 September, 2016. The first 200 search results were screened for potential further inclusion in the review. All peer-review papers that contained mention of environmental characteristics at hydropower facilities in the paper title, abstract, or executive summary in addition to papers that contained terms that signaled potential relevance to this project were retained for further review. An example of the type of paper that signaled potential relevance to this project that was retained would be a paper that discussed watershed land use change over a period of time because hydropower development and reservoir inundation is sometimes calculated in land use change statistics. Likewise, papers that discussed the response of an organism or group of organisms to flow or river regulation were retained for further review even if hydropower was not specifically mentioned. Papers that were further included in the review were entered into Mendeley (https://www.mendeley.com) — an open access web-tool for managing references and PDFs of literature. Papers that could not be definitively included or excluded based on the title and abstract were also retained for further review. Papers not including information on the environmental effects of hydropower were excluded and a Mendeley folder was created to document the citations of peer-reviewed papers that were screened but not included in the catalog. In this way, interested parties can gain a better understanding of why a study was or was not included in the review.

\section{Database creation}

Papers retained were searched for metrics characterizing the environmental effects of hydropower as well as for other information relevant to the metric such as values, methods of data collection, and temporal resolution of the metric for incorporation into the catalog using data in tables, figures, and text of papers (Appendix A). Metrics were considered as any means, either quantitative or qualitative, of characterizing the environment at, near, or associated with a hydropower plant. The database of metrics was then georeferenced and related to the NHAAP or GRanD hydropower databases where possible to create a spatially-related database of 
hydropower environmental characteristics. A bibliography of papers that passed the initial review filter but did not contain metrics relevant to this study are provided in Appendix B.

\section{Catalog creation}

To gain an understanding of what metrics have been most commonly used and most useful for describing the environmental effects of hydropower, a catalog indexed by metric was created. This catalog also contained summaries of metric usage including the number of times, locations, and studies that used each metric as well as a narrative description. Summarized metrics were also then coded with unique identifiers corresponding to metric type, category, and number.

\section{Data analysis}

All data analysis was conducted in R (Version 3.2.2, "Fire Safety"). A kappa statistic ( $\kappa)$ was calculated on a subset of $25 \%$ search results to quantify consistency in study inclusion between two article reviewers (ORNL staff Dr. Brenda Pracheil and Dr. Ryan McManamay), calculated as

$$
\kappa=\left(p_{\mathrm{o}}-\mathrm{p}_{\mathrm{e}}\right) /\left(1-\mathrm{p}_{\mathrm{e}}\right)
$$

where $\mathrm{p}_{\mathrm{o}}$ is the observed agreement of article inclusion among those rating articles and pe is the probability of random agreement between reviewers calculated from the data collected. Reviewers in complete agreement would yield a $\kappa=1$ and agreement no greater than that expected by random chance $\left(p_{e}\right)$ would yield a $\kappa<0$. A $\kappa>0.50$ was required between a pair of reviewers before searches were concluded to be of sufficient consistency. The k-value between the two in this study was 0.86 .

\section{LITERATURE CITED}

Collaboration for Environmental Evidence (CEE) (2013). Guidelines for systematic review and evidence synthesis in environmental management version 4.2. Environmental evidence: http://devtestsite.co.uk/cee/wp-content/uploads/ 2014/06/Review-guidelines-version-4.2final.pdf 
Table 1. Search terms forming initial search strings for systematic review. Each search string was comprised of one environmental term and one hydropower term. Quotations around compound terms such as "flow regime" or "stilling basin" were used to help restrict search results to those relevant to this review. Asterisks were used to include multiple forms of words. For example, "alter*" would search for "altered", "alteration", "alters", etc.

\begin{tabular}{|c|c|}
\hline Environmental Term & Hydropower Term \\
\hline Alter* & Conveyance \\
\hline Assess* & "Dam* OR Barrage*" \\
\hline Biodiversity & "Hydropower OR Hydroelectric" \\
\hline Biot* & Infrastructure \\
\hline "Communit* OR Community" & Powerhouse \\
\hline “Connect* OR Connectivity” & "Reservoir* OR Impound*” \\
\hline Effect* & "Tailrace* OR Tailwater*” \\
\hline Environment* & Turbine \\
\hline Fish* & \\
\hline Flow* & \\
\hline "Flow regime*" & \\
\hline "Fragment* OR Fragmentation" & \\
\hline Geomorph* & \\
\hline Impact* & \\
\hline "Land cover" & \\
\hline Limnolog* & \\
\hline Macroinvert* & \\
\hline Macrophyte* & \\
\hline Measur* & \\
\hline Metric* & \\
\hline Mussel* & \\
\hline Population & \\
\hline Quantif* & \\
\hline Sediment* & \\
\hline Sustain* & \\
\hline "Water quality" & \\
\hline "Water quantity" & \\
\hline
\end{tabular}




\section{APPENDIX B. BIBLIOGRAPY OF PEER-REVIEW LITERATURE INCLUDED IN THE DATABASE}

Appendix B. Papers in systematic review classified as relevant to environmental effects of hydropower and had metrics that were included in the metric database and catalog. As of 10 January 10, 2017, there were 97 studies included in the database.

Agostinho, A. A., Pelicice, F. M., \& Gomes, L. C. (2008). Dams and the fish fauna of the Neotropical region: impacts and management related to diversity and fisheries. Brazilian Journal of Biology, 68(4), 1119-1132.

Alonso-Gonzalez, C., \& Gort??zar, J. a. (2008). Dam function rules based on brown trout flow requirements: Design of environmental flow regimes in regulated streams. Hydrobiologia, 609(1), 253-262.

Anderson, E. P., \& Pringle, C. M. (2008). Quantifying the extent of river fragmentation by hydropower dams in the Sarapiqui River Basin, Costa Rica. Aquatic Conservation: Marine and Freshwater Ecosystems, 18(4), 408--417.

Andriolo, A., Piovezan, U., Costa, M. J. R., Torres, H. A., Vogliotti, A., Zerbini, A. N., \& Duarte, J. M. B. (2013). Severe population decline of marsh deer, Blastocerus dichotomus (Cetartiodactyla: Cervidae), a threatened species, caused by flooding related to a hydroelectric power plant. Zoologia, 30(6), 630-638.

Arias, M. E., Cochrane, T. A., Lawrence, K. S., Killeen, T. J., \& Farrell, T. A. (2011). Paying the forest for electricity: a modelling framework to market forest conservation as payment for ecosystem services benefiting hydropower generation. Environmental Conservation, 38(04), 473-484.

Armanini, D. G., Chaumel, A. I., Monk, W. A., \& Marty, J. (2014). Benthic macroinvertebrate flow sensitivity as a tool to assess effects of hydropower related ramping activities in streams in Ontario (Canada). Ecological, 46, 466--476.

Arnekleiv, J. V., Kraabl, M., \& Museth, J. (2007). Efforts to aid downstream migrating brown trout (Salmo trutta L.) kelts and smolts passing a hydroelectric dam and a spillway. Hydrobiologia, 582(1), 5--15.

Bacheler, N. M., Neal, J. W., \& Noble, R. L. (2004). Reproduction of a landlocked diadromous fish population: bigmouth sleepers Gobiomorus dormitor in a reservoir in Puerto Rico. Caribbean Journal of Science, 40(2), 223-231.

Bain, M. B., Finn, J. T., Booke, H. E., \& Brain, M. B. a. (1988). Streamflow Regulation And Fish Community Structure. Ecology, 69, 382-392.

Bambace, L. A. W., Ramos, F. M., Lima, I. B. T., \& Rosa, R. R. (2007). Mitigation and recovery of methane emissions from tropical hydroelectric dams. Energy, 32(6), 1038-1046. 
Bardossy, A., \& Molnr, Z. (2004). Statistical and geostatistical investigations into the effects of the Gabcikovo hydropower plant on the groundwater resources of northwest Hungary/Analyses. Hydrological Sciences Journal, 49(4), 623.

Bartholow, J. M., Campbell, S. G., \& Flug, M. (2004). Predicting the thermal effects of dam removal on the Klamath River. Environmental Management, 34(6), 8856-8874.

Bastien, J., Demarty, M., \& Tremblay, A. (2011). CO2 and CH4 diffusive and degassing fluxes from 2003 to 2009 at Eastmain 1 reservoir, Qu \' e bec, Canada. Inland Waters, 1(2), 113123.

Bates, J. M. (1962). The impact of impoundment on the mussel fauna of Kentucky Reservoir, Tennessee River. American Midland Naturalist, 68(1), 232-236.

Beamesderfer, R. C., \& Rieman, B. E. (1990). Management implications of a model of predation by a resident fish on juvenile salmonids migrating through a Columbia River reservoir. North American Journal of Fisheries Managemen, 10(3), 290-304.

Beghelli, F. G. S., Santos, A. C. A., \& Urso-Guimares, M. V. (2012). Relationship between space distribution of the benthic macroinvertebrates community and trophic state in a Neotropical reservoir (Itupararanga, Brazil). Biota, 12(4), 114-124.

Beilfuss, R. (2010). Modelling trade-offs between hydropower generation and environmental flow scenarios: a case study of the Lower Zambezi River Basin, Mozambique. International Journal of River Basin Management, 8(3-4), 331-347.

Bell, L. M. (1985). A Fish Passage Problem at the Seton Hydroelectric Project in Southwestern British Columbia. Canadian Water Resources Journal, 10(1), 32-39.

Benchimol, M., \& Venticinque, E. M. (2014). Responses of Primates to Landscape Change in Amazonian Land-bridge islands-a Multi-scale Analysis. Biotropica, 46(4), 470-478.

Benejam, L., Saura-Mas, S., Bardina, M., \& Sol. (2014). Ecological impacts of small hydropower plants on headwater stream fish: from individual to community effects. Ecology of Freshwater Fish.

Benjankar, R., Jorde, K., Yager, E. M., \& Egger, G. (2012). The impact of river modification and dam operation on floodplain vegetation succession trends in the Kootenai River, USA. Ecological, 46, 88-97.

Benn, P. C., \& Erskine, W. D. (1994). Complex channel response to flow regulation: Cudgegong River below Windamere Dam, Australia. Applied Geography, 14(2), 153-168.

Bennett, A. M., \& Keevil, M. (2010). Spatial ecology and population genetics of northern map turtles (Graptemys geographica) in fragmented and continuous habitats in Canada. Conservation and Biology, 9(2), 185-195. 
Bergman, N., Sholker, O., Roskin, J., \& Greenbaum, N. (2014). The Nahal Oz Reservoir dambreak flood: geomorphic impact on a small ephemeral loess-channel in the semi-arid Negev Desert, Israel. Geomorphology, 201, 83-97.

Berkes, F. (1982). Preliminary impacts of the James Bay hydroelectric project, Quebec, on estuarine fish and fisheries. Arctic, 35(4), 524-530.

Bhatt, R., \& Khanal, S. N. (2010). Vegetation analysis and differences in local environment variables in indrawati hydropower project areas in Nepal. International Research Journal: Plant Science, 14(83-94).

Bhatt, R. P., \& Khanal, S. N. (2012). A study on change in flow regime and discharge impacts on water quality of hydropower operation. International Journal of Ecology and Development, 21, 76-88.

Bhatt, R. P., Khanal, S. N., \& Maskey, R. K. (2011). Water Quality Impacts of Hydropower Project Operation in Bhotekoshi River Basin Sindhulpalchowk District in Nepal. ijpaes.com, 1(1), 88-101.

Bhutiani, R., Khanna, D. R., \& Tyagi, P. K. (2014). Application of CCME WQI to evaluate feasibility of potable water availability: A case study of Tehri dam reservoir. Environment Conservation Journal, 15(1-2), 13-19.

Bini, L. M., Thomaz, S. M., \& Murphy, K. J. (1999). Aquatic macrophyte distribution in relation to water and sediment conditions in the Itaipu Reservoir, Brazil. Biology, Ecology and Management of Aquatic Plants, 147, 147-154.

Black, A. R., Rowan, J. S., Duck, R. W., Bragg, O. M., \& Clelland, B. E. (2005). DHRAM: A method for classifying river flow regime alterations for the EC Water Framework Directive. Aquatic Conservation: Marine and Freshwater Ecosystems, 15(5), 427-446.

Bond, W. J., Coe, N., \& Jackson, P. B. N. (1978). The limnology of Cabora Bassa, Moçambique, during its first year. Freshwater Biology, 8(5), 433-447.

Branco, P., Segurado, P., Santos, J. M., \& Pinheiro, P. (2012). Does longitudinal connectivity loss affect the distribution of freshwater fish? Ecological Engineering, 48, 70-78.

Bravard, J. P., Landon, N., Peiry, J. L., \& Pigay, H. (1999). Principles of engineering geomorphology for managing channel erosion and bedload transport, examples from French rivers. Geomorphology, 31(1-4), 291-311.

Budhu, M., Contractor, D. N., \& Wu, C. S. (1994). Modelling groundwater changes due to fluctuating dam discharge. Applied Mathematical Modelling, 18(12), 665-671. 
Calles, O., Karlsson, S., Vezza, P., \& Comoglio, C. (2013). Success of a low-sloping rack for improving downstream passage of silver eels at a hydroelectric plant. Freshwater Biology, 58(10), 2168-2179.

Callisto, M., Goulart, M., \& Barbosa, F. A. R. (2005). Biodiversity assessment of benthic macroinvertebrates along a reservoir cascade in the lower Sao Francisco river (northeastern Brazil). Brazilian Journal of Biology, 65(2), 229-240.

Carley, J. K., Pasternack, G. B., Wyrick, J. R., \& Barker, J. R. (2012). Significant decadal channel change 58-67years post-dam accounting for uncertainty in topographic change detection between contour maps and point cloud models. Geomorphology, 179, 71-88.

Chicharo, L., Chicharo, M. A., \& Ben-Hamadou, R. (2006). Use of a hydrotechnical infrastructure (Alqueva Dam) to regulate planktonic assemblages in the Guadiana estuary: basis for sustainable water and ecosystem services. Estuarine, Coastal and Shelf Science, 70(1-2), 3-18.

Chiu, M. C., Yeh, C. H., Sun, Y. H., \& Kuo, M. H. (2013). Short-term effects of dam removal on macroinvertebrates in a Taiwan stream. Aquatic Ecology, 47(2), 245-252.

Churchill, C. J. (2013). Spatio-temporal spawning and larval dynamics of a zebra mussel (Dreissena polymorpha) population in a North Texas Reservoir: implications for invasions in. Aquatic Invasions, 8(4), 389-406.

Craven, S. W., Peterson, J. T., Freeman, M. C., Kwak, T. J., \& Irwin, E. (2010). Modeling the relations between flow regime components, species traits, and spawning success of fishes in warmwater stream. Environmental Management, 46(2), 181-194.

Dai, Z., Chu, A., Stive, M., Du, J., \& Li, J. (2011). Is the Three Gorges Dam the cause behind the extremely low suspended sediment discharge into the Yangtze (Changjiang) Estuary of 2006? Hydrological Sciences Journal, 56(7), 1280-1288.

Dauble, D. D. (1986). Life history and ecology of the largescale sucker (Castostomus macrocheilus) in the Columbia River. American Midland Naturalist, 116(2), 356-367.

de Almeida, A. T., Moura, P. S., \& Marques, A. S. (2005). Multi-impact evaluation of new medium and large hydropower plants in Portugal centre region. Renewable and Sustainable Energy Reviews, 9(2), 149-167.

de Almeida, F. S. a. (2003). Population structure analysis of Pimelodus maculatus (Pisces, Siluriformes) from the Tiet and Paranapanema Rivers (Brazil). Genetics and Molecular Biology, 26(3), 301-305.

Dean, D. J., \& Schmidt, J. C. (2013). The geomorphic effectiveness of a large flood on the Rio Grande in the Big Bend region: Insights on geomorphic controls and post-flood geomorphic response. Geomorphology, 201, 183-198. 
Duchemin, E., Lucotte, M., \& Canuel, R. (1995). Production of the greenhouse gases CH4 and $\mathrm{CO} 2$ by hydroelectric reservoirs of the boreal region. Global Biogeochemical Cycles, 9(4), 529-540.

Ebel, W. J. (1969). Supersaturation of nitrogen in the Columbia River and its effect on salmon and steelhead trout. Fishery Bulletin, 68(1), 1-11.

Effler, S. W., Perkins, M. G., \& Johnson, D. L. (1998). The optical water quality of Cannonsville Reservoir: Spatial and temporal patterns, and the relative roles of phytoplankton and inorganic tripton. Lake and Reservoir Management, 14(2-3), 238-253.

Englund, D., Brunberg, A., \& Jacks, G. (2008). A case study of a freshwater pearl mussel (Margaritifera margaritifera) population in central Sweden. Geografiska Annaler. Series A, Physical, 90(4), 251-258.

Foster, B. R., \& Rahs, E. Y. (1985). A study of canyon-dwelling mountain goats in relation to proposed hydroelectric development in northwestern British Columbia, Canada. Biological Conservation, 33(3), 209-228.

Froebrich, J., Bauer, M., Ikramova, M., \& Olsson, O. (2007). Water quantity and quality dynamics of the THC-Tuyamuyun Hydroengineering Complex-And implications for reservoir operation. Environmental Science and Pollution Research-- International, 14(6), 435-442.

Gain, A. K., Apel, H., Renaud, F. G., \& Giupponi, C. (2013). Thresholds of hydrologic flow regime of a river and investigation of climate change impact - the case of the Lower Brahmaputra river Basin. Climatic Change, 120(1), 463--475.

Galbraith, H. S., Blakeslee, C. J., \& Lellis, W. A. (2015). Behavioral responses of freshwater mussels to experimental dewatering. Freshwater Science, 34(1), 42--52.

Galbraith, H. S., \& Vaughn, C. C. (2009). Temperature and food interact to influence gamete development in freshwater mussels. Hydrobiologia, 636(1), 35--47.

Gelwick, F. P., \& Matthews, W. J. (1990). Temporal and spatial patterns in littoral-zone fish assemblages of a reservoir (Lake Texoma, Oklahoma-Texas, USA). Environmental Biology of Fishes, 27(2), 107--120.

Gobo, A. E., Etiga, G. E., \& Amangabara, G. T. (2014). Flow effect of Kainji Dam on the Distribution of Water Hyacinth in Kolo Creek, Bayelsa State of Nigeria. International Journal of Environment and Resource, 3(2).

Graf, W. L. (2006). Downstream hydrologic and geomorphic effects of large dams on American rivers. Geomorphology, 79(3-4), 336-360. 
Grill, G., Dallaire, C. O., Chouinard, E. F., \& Sindorf, N. (2014). Development of new indicators to evaluate river fragmentation and flow regulation at large scales: a case study for the Mekong River Basin. Ecological Applications, 45, 148-159.

Guo, Z., Xiao, X., \& Li, D. (2000). An assessment of ecosystem services: water flow regulation and hydroelectric power production. Ecological Applications, 10(3), 925-936.

Hay, C. H., Franti, T. G., Marx, D. B., Peters, E. J., \& Hesse, L. W. (2008). Macroinvertebrate drift density in relation to abiotic factors in the Missouri River. Hydrobiologia, 598(1), 175-189.

Heidari, A., Mousavi-Sabet, H., Khoshkholgh, M., Esmaeili, H. R., \& Eagderi, S. (2013). The impact of Manjil and Tarik dams (Sefidroud River, southern Caspian Sea basin) on morphological traits of Siah Mahi Capoeta gracilis (Pisces: Cyprinidae). International Journal of Aquatic Biology, 1(4), 195-201.

Hughes, J. S., Deng, Z. D., Weiland, M. A., \& Martinez, J. J. (2011). Water Velocity Measurements on a Vertical Barrier Screen at the Bonneville Dam Second Powerhouse. Energies, 4(11), 2038-2048.

Humborg, C., Pastuszak, M., Aigars, J., \& Siegmund, H. (2006). Decreased silica land-sea fluxes through damming in the Baltic Sea catchment-significance of particle trapping and hydrological alterations. Biogeochemistry, 77(2), 265-281.

Huo, J. X., Song, H. Z., \& Luo, L. (2015). Investigation of groundwater chemistry at a dam site during its construction: a case study of Xiangiiaba Dam, China. Environmental Earth Sciences, 74(3), 2451-2461.

Huraut, J. P., Cazaillet, O., \& Ducos, X. (2002). Ambuklao hydroelectric scheme-sedimentation of the reservoir and rehabilitation program. In Dam Maintenance and Rehabilitation: Proceedings of the International Congress on Conservation and Rehabilitation of Dams, Madrid, 11-13 November 2002 (p. 449). CRC Press.

Istvnovics, V., Honti, M., Vrs, L., \& Kozma, Z. (2010). Phytoplankton dynamics in relation to connectivity, flow dynamics and resource availability - the case of a large, lowland river, the Hungarian Tisza. Hydrobiologia, 637(1), 121-141.

Jepsen, N., Aarestrup, K., \& kland, F. (1998). Survival of radio-tagged Atlantic salmon (Salmo salar L.) and trout (Salmo trutta L.) smolts passing a reservoir during seaward migration. Advances in Invertebrates and Fish Telemetry, 130, 347-353.

Jones, J., Ahlstedt, S., Ostby, B., \& Beaty, B. (2014). Clinch River freshwater mussels upstream of Norris Reservoir, Tennessee and Virginia: a quantitative assessment from 2004 to 2009. JAWRA Journal of the American Water Resources Association, 50(4), 820-836. 
Kaster, J. L., \& Jacobi, G. Z. (1978). Benthic macroinvertebrates of a fluctuating reservoir. Freshwater Biology, 8(3), 283-290.

Kemenes, A., \& Forsberg, B. R. (2007). Methane release below a tropical hydroelectric dam. Geophysical research, 34(12).

Klaver, G., van Os, B., Negrel, P., \& Petelet-Giraud, E. (2007). Influence of hydropower dams on the composition of the suspended and riverbank sediments in the Danube. Environmental Pollution, 148(3), 718-728.

Kotut, K., Krienitz, L., \& Muthuri, F. M. (1998). Temporal changes in phytoplankton structure and composition at the Turkwel Gorge Reservoir, Kenya. Hydrobiologia, 368(1), 41-59.

Kumar, A., \& Sharma, M. P. (2016). A modeling approach to assess the greenhouse gas risk in Koteshwar hydropower reservoir, India. Human and Ecological Risk Assessment:, 22(8), 1651-1664.

Laine, A., Kamula, R., \& Hooli, J. (1998). Fish and lamprey passage in a combined Denil and vertical slot fishway. Fisheries Management and, 5(1), 31-44.

Larinier, M. (2008). Fish passage experience at small-scale hydro-electric power plants in France. Hydrobiologia, 609(1), 97-108.

Lehman, J. T. (2011). Nuisance cyanobacteria in an urbanized impoundment: interacting internal phosphorus loading, nitrogen metabolism, and polymixis. Hydrobiologia, 661(1), 277287.

Ma, Q., Liang, R., Li, R., Feng, J., \& Li, K. (2016). Operational regulation of water replenishment to reduce supersaturated total dissolved gas in riverine wetlands. Ecological Engineering, 96, 162-169.

Malini, B. H., \& Rao, K. N. (2004). Coastal erosion and habitat loss along the Godavari delta front- a fallout of dam construction. Current Science, 87(9), 1232-1236.

Meile, T., Boillat, J. L., \& Schleiss, A. J. (2011). Hydropeaking indicators for characterization of the Upper-Rhone River in Switzerland. Aquatic Sciences, 73(1), 171-182.

Milošković, A., Branković, S., Simić, V., Kovačević, S., Ćirković, M., \& Manojlović, D. (2013). The accumulation and distribution of metals in water, sediment, aquatic macrophytes and fishes of the Gruza Reservoir, Serbia. Bulletin of environmental, 90(5), 563-569.

Mims, M. C., \& Olden, J. D. (2013). Fish assemblages respond to altered flow regimes via ecological filtering of life history strategies. Freshwater Biology, 58(1), 50-62. 
Mistak, J. L., Hayes, D. B., \& Bremigan, M. T. (2003). Food habits of coexisting salmonines above and below Stronach Dam in the Pine River, Michigan. Environmental Biology of Fishes, 67(2), 179-190.

Muir, W. D., Smith, S. G., \& Williams, J. G. (2001). Survival estimates for migrant yearling Chinook salmon and steelhead tagged with passive integrated transponders in the lower Snake and lower Columbia rivers. North American Journal of Fisheries Management, 21(2), 269-282.

Politano, M., Amado, A. A., Bickford, S., \& Murauskas, J. (2012). Evaluation of operational strategies to minimize gas supersaturation downstream of a dam. Computers \& Fluids, $68,168-185$.

Ribi, J. M., Boillat, J. L., Peter, A., \& Schleiss, A. J. (2014). Attractiveness of a lateral shelter in a channel as a refuge for juvenile brown trout during hydropeaking. Aquatic Sciences, $76(4), 527-541$.

Ribolli, J., Melo, C. M. R., \& Zaniboni-Filho, E. (2012). Genetic characterization of the neotropical catfish Pimelodus maculatus (Pimelodidae, Siluriformes) in the Upper Uruguay River. Genetics and molecular biology, 35(4), 761-769.

Scruton, D. A., Pennell, C. J., \& Robertson, M. J. (2005). Seasonal response of juvenile Atlantic salmon to experimental hydropeaking power generation in Newfoundland, Canada. North American Journal of Fisheries Management, 25(3), 964-974.

Smith, N. D., Morozova, G. S., Prez-Arlucea, M., \& Gibling, M. R. (2016). Dam-induced and natural channel changes in the Saskatchewan River below the EB Campbell Dam, Canada. Geomorphology, 269, 186-202.

Soltani, F., Kerachian, R., \& Shirangi, E. (2010). Developing operating rules for reservoirs considering the water quality issues: Application of ANFIS-based surrogate models. Expert Systems with Applications, 37(9), 6639-6645.

Song, K., Yan, E., Zhang, G., Lu, S., \& Yi, Q. (2015). Effect of hydraulic properties of soil and fluctuation velocity of reservoir water on landslide stability. Environmental Earth Sciences. 74(6), 5319-5329

Stevens, L. E., Schmidt, J. C., \& Ayers, T. J. (1995). Flow regulation, geomorphology, and Colorado River marsh development in the Grand Canyon, Arizona. Ecological Applications, 5(4), 1025-1039.

Tamene, L., Park, S. J., \& Dikau, R. (2006). Reservoir siltation in the semi-arid highlands of northern Ethiopia: sediment yield-catchment area relationship and a semi-quantitative approach for predicting. Earth Surface Processes, 31(11), 1364-1383. doi:10.1002/esp.1338 
Thomaz, S. M., Carvalho, P., Mormul, R. P., \& Ferreira, F. A. (2009). Temporal trends and effects of diversity on occurrence of exotic macrophytes in a large reservoir. Acta Oecologica, 35(5), 614-620.

Thompson, L. C., Cocherell, S. A., \& Chun, S. N. (2011). Longitudinal movement of fish in response to a single-day flow pulse. Environmental Biology of, 90(3), 253-261.

Tufford, D. L., \& McKellar, H. N. (1999). Spatial and temporal hydrodynamic and water quality modeling analysis of a large reservoir on the South Carolina (USA) coastal plain. Ecological Modelling, 114(2-3), 137-173. 


\section{APPENDIX C. SUMMARY OF STUDY LOCATIONS AND ENVIRONMENTAL METRIC CATEGORIES EXTRACTED FROM THE 97 PEER-REVIEWED JOURNAL ARTICLES}

\begin{tabular}{|c|c|c|c|c|c|c|}
\hline $\begin{array}{l}\text { Reference } \\
\text { Article }\end{array}$ & Continent & $\begin{array}{l}\text { Country } \\
\text { (or } \\
\text { countries) }\end{array}$ & $\begin{array}{l}\text { Dam(s) or Study } \\
\text { Location }\end{array}$ & River(s) & Metrics & $\begin{array}{l}\text { Cate- } \\
\text { gories }\end{array}$ \\
\hline $\begin{array}{l}\text { Agostinho_e } \\
\text { tAl_2008 } \\
{[23]}\end{array}$ & S. America & $\begin{array}{l}\text { Brazil, } \\
\text { Paraguay }\end{array}$ & $\begin{array}{l}\text { Tres Irmaos, Corumba, } \\
\text { Itaipu, and Porto } \\
\text { Primavera Dams }\end{array}$ & $\begin{array}{l}\text { Corumba, } \\
\text { Parana, } \\
\text { Tiete, } \\
\text { Parana } \\
\text { Yacyreta, } \\
\text { Parana }\end{array}$ & 4 & BB \\
\hline $\begin{array}{l}\text { AlonsoGonz } \\
\text { alez_etAl_2 } \\
008 \text { [24] }\end{array}$ & Europe & Spain & Proposed dam & Tormes & 26 & $\begin{array}{l}\mathrm{BB}, \\
\mathrm{W} 1\end{array}$ \\
\hline $\begin{array}{l}\text { Anderson_et } \\
\text { al_2008 } \\
{[25]}\end{array}$ & S. America & Costa Rica & $\begin{array}{l}\text { Suerkata, Toro I, Toro } \\
\text { II, Volcan, Segundo, } \\
\text { El Angel, Dona Julia, } \\
\text { Cariblanco, and Don } \\
\text { Pedro Dams }\end{array}$ & $\begin{array}{l}\text { Sarapiqui, } \\
\text { Toro, } \\
\text { Volcan, } \\
\text { Agnel, } \\
\text { Tuolumne }\end{array}$ & 9 & W1 \\
\hline $\begin{array}{l}\text { Andriolo_et } \\
\text { Al_2013 } \\
{[2 \overline{6}]}\end{array}$ & S. America & Brazil & Porto Primovera Dam & Paraná & 4 & $\mathrm{BB}$ \\
\hline $\begin{array}{l}\text { Arias_etAl_ } \\
2011[27]\end{array}$ & Asia & Cambodia & Proposed dam & Mekong & 6 & $\begin{array}{l}\mathrm{GM} \\
\mathrm{LC}\end{array}$ \\
\hline $\begin{array}{l}\text { Armanini_et } \\
\text { Al_2014 } \\
{[28]}\end{array}$ & N. America & Canada & $\begin{array}{l}\text { Steephill Falls } \\
\text { Generating Station }\end{array}$ & $\begin{array}{l}\text { Magpie, } \\
\text { Batchawan } \\
\text { a }\end{array}$ & 3 & $\mathrm{BB}$ \\
\hline $\begin{array}{l}\text { Arnekleiv_e } \\
\text { tAl_2007 } \\
{[29]}\end{array}$ & Europe & Norway & Mjosa Dam & $\begin{array}{l}\text { Gudbrands } \\
\text { dalslågen }\end{array}$ & 5 & $\begin{array}{l}\text { BB, } \\
\text { W1, } \\
\text { W2 }\end{array}$ \\
\hline $\begin{array}{l}\text { Bacheler_et } \\
\text { Al_2004 } \\
\text { [30] }\end{array}$ & N. America & $\begin{array}{l}\text { United } \\
\text { States }\end{array}$ & Carite Dam & La Plata & 4 & $\mathrm{BB}$ \\
\hline $\begin{array}{l}\text { Bain_etAl_1 } \\
988[31]\end{array}$ & N. America & $\begin{array}{l}\text { United } \\
\text { States }\end{array}$ & $\begin{array}{l}\text { Stream reach flow } \\
\text { modified by four dams } \\
\text { (no names provided) }\end{array}$ & $\begin{array}{l}\text { Connecticu } \\
\mathrm{t}\end{array}$ & 12 & $\begin{array}{l}\mathrm{BB}, \\
\mathrm{GM}\end{array}$ \\
\hline $\begin{array}{l}\text { Bambace_et } \\
\text { al_2007[32] }\end{array}$ & S. America & Brazil & $\begin{array}{l}\text { Serra da Mesa and } \\
\text { Tucurui Dams }\end{array}$ & Toncatins & 3 & W1 \\
\hline $\begin{array}{l}\text { Bardossy_et } \\
\text { al_2004[33] }\end{array}$ & Europe & Slovakia & Gabicikovo Dam & Danube & 18 & W1 \\
\hline $\begin{array}{l}\text { Bartholow_- } \\
\text { etal_2004 } \\
{[34]}\end{array}$ & N. America & $\begin{array}{l}\text { United } \\
\text { States }\end{array}$ & $\begin{array}{l}\text { Iron Gate, John C. } \\
\text { Boyle, Keno, and } \\
\text { Copco Dams }\end{array}$ & Klamath & 20 & W1 \\
\hline $\begin{array}{l}\text { Bastien_etA } \\
1 \text { 1_2011 [35] }\end{array}$ & N. America & Canada & Sarcelle Dam & Eastmain & 4 & W2 \\
\hline $\begin{array}{l}\text { Bates_1962 } \\
{[36]}\end{array}$ & N. America & $\begin{array}{l}\text { United } \\
\text { States }\end{array}$ & Kentucky Dam & Tennessee & 2 & $\mathrm{BB}$ \\
\hline
\end{tabular}




\begin{tabular}{|c|c|c|c|c|c|c|}
\hline $\begin{array}{l}\text { Beamesderf } \\
\text { er_et_al_19 } \\
90[37]\end{array}$ & N. America & $\begin{array}{l}\text { United } \\
\text { States }\end{array}$ & John Day Dam & Columbia & 22 & W1 \\
\hline $\begin{array}{l}\text { Beghelli_et } \\
\text { Al_2012 } \\
{[38]}\end{array}$ & S. America & Brazil & Itupararanga Dam & Sorocaba & 9 & $\begin{array}{l}\text { BB, } \\
\text { W2 }\end{array}$ \\
\hline $\begin{array}{l}\text { Beilfuss_et } \\
\text { al_1994[39] }\end{array}$ & Oceania & Australia & Windamere Dam & Cudgegong & 12 & W1 \\
\hline $\begin{array}{l}\text { Bell_1985 } \\
{[40]}\end{array}$ & N. America & Canada & Seton Dam & Seton & 2 & W1 \\
\hline $\begin{array}{l}\text { Benchimol_- } \\
\text { et_al_2014 } \\
\text { [41] }\end{array}$ & S. America & Brazil & Balbina Dam & Uatuma & 6 & W1 \\
\hline $\begin{array}{l}\text { Benejam_et } \\
\text { Al_2016 } \\
{[4 \overline{2}]}\end{array}$ & Europe & Spain & $\begin{array}{l}\text { Sixteen small } \\
\text { hydropower plants: } \\
\text { Brutau 1, Brutau 2, } \\
\text { Pardines, El Molí } \\
\text { Rialp, Feitús, } \\
\text { Cruanyes, Molí de } \\
\text { Sart, Matabosch, } \\
\text { Montagut, Molí Gran } \\
\text { Pont Vell, Cal Gat, } \\
\text { Surribes, L'Escala, La } \\
\text { Cubia, Fàbrica Tomàs, } \\
\text { Crous }\end{array}$ & Upper Ter & 9 & $\begin{array}{l}\mathrm{BB}, \\
\mathrm{GM}\end{array}$ \\
\hline $\begin{array}{l}\text { Benjankar_e } \\
\text { tAl_2012- } \\
{[42]}\end{array}$ & N. America & $\begin{array}{l}\text { United } \\
\text { States }\end{array}$ & Libby Dam & Kootenai & 3 & $\begin{array}{l}\mathrm{BB}, \\
\mathrm{W} 1\end{array}$ \\
\hline $\begin{array}{l}\text { Benn_et_al_ } \\
1994[43]\end{array}$ & Africa & $\begin{array}{l}\text { Zambia, } \\
\text { Zimbabwe, } \\
\text { Mozambiq } \\
\text { ue }\end{array}$ & $\begin{array}{l}\text { Kariba, Kafue George, } \\
\text { and Cahora Bassa } \\
\text { Dams }\end{array}$ & $\begin{array}{l}\text { Zambezi, } \\
\text { Kafue }\end{array}$ & 9 & W1 \\
\hline $\begin{array}{l}\text { BennettAnd } \\
\text { Keevil_201 } \\
0 \text { [44] }\end{array}$ & N. America & Canada & $\begin{array}{l}\text { Trent-Severn } \\
\text { Waterway locks and } \\
\text { dams }\end{array}$ & $\begin{array}{l}\text { Trent- } \\
\text { Severn } \\
\text { Waterway }\end{array}$ & 1 & $\mathrm{BB}$ \\
\hline $\begin{array}{l}\text { Bergman_et } \\
\text { al_2014 } \\
\text { als] }\end{array}$ & Asia & Israel & Nahal Oz Dam & $\begin{array}{l}\text { Nahal } \\
\text { Yare'akh } \\
\text { River }\end{array}$ & 9 & W1 \\
\hline $\begin{array}{l}\text { Berkes_198 } \\
2[46]\end{array}$ & N. America & Canada & La Grande 1 Dam & La Grande & 5 & $\mathrm{BB}$ \\
\hline $\begin{array}{l}\text { Bhatt_and } \\
\text { Khanal_201 } \\
0[47]\end{array}$ & Asia & Nepal & Indrawati III Dam & Indrawati & 15 & $\begin{array}{l}\text { W1, } \\
\text { W2 }\end{array}$ \\
\hline $\begin{array}{l}\text { Bhatt_et_al_- } \\
2011 \text { [48] }\end{array}$ & Asia & Nepal & $\begin{array}{l}\text { Upper Bhotekoshi } \\
\text { Hydropower Project }\end{array}$ & Bhotekoshi & 19 & W1 \\
\hline $\begin{array}{l}\text { BhattAndKh } \\
\text { anal_2012 } \\
\text { [49] }\end{array}$ & Asia & Nepal & $\begin{array}{l}\text { Upper Bhotekoshi } \\
\text { Hydropower Project }\end{array}$ & Bhotekoshi & 18 & $\begin{array}{l}\text { W1, } \\
\text { W2 }\end{array}$ \\
\hline $\begin{array}{l}\text { Bhutiani_et } \\
\text { Al_2014 } \\
\text { [50] }\end{array}$ & Asia & India & Tehri Dam & Bhagirathi & 20 & W2 \\
\hline
\end{tabular}




\begin{tabular}{|c|c|c|c|c|c|c|}
\hline $\begin{array}{l}\text { Bini_etAl_1 } \\
999 \text { [51] }\end{array}$ & S. America & Paraguay & Itaipu Dam & $\begin{array}{l}\text { Parana } \\
\text { Yacyreta }\end{array}$ & 13 & $\begin{array}{l}\mathrm{BB}, \\
\mathrm{W} 1\end{array}$ \\
\hline $\begin{array}{l}\text { Black_et_al } \\
\text { _2005[52] }\end{array}$ & Europe & Scotland & Megget Dam & $\begin{array}{l}\text { Allt a' } \\
\text { Chireachai } \\
\text { n, Megget }\end{array}$ & 333 & $\begin{array}{l}\text { BB, } \\
\text { CF, } \\
\text { GM, } \\
\text { ID, LC, } \\
\text { W1, } \\
\text { W2 }\end{array}$ \\
\hline $\begin{array}{l}\text { Bond_et_al } \\
1978[53]\end{array}$ & Africa & $\begin{array}{l}\text { Mozambiq } \\
\text { ue }\end{array}$ & Cahora Bassa Dam & Zambezi & 53 & $\begin{array}{l}\text { BB, } \\
\text { GM, } \\
\text { ID, LC, } \\
\text { W1, } \\
\text { W2 }\end{array}$ \\
\hline $\begin{array}{c}\text { Branco_etAl } \\
2012[54]\end{array}$ & Europe & Portugal & $\begin{array}{l}\text { A total of } 196 \text { stream } \\
\text { sampling sites in three } \\
\text { river basins. }\end{array}$ & $\begin{array}{l}\text { Tagus, } \\
\text { Mondego, } \\
\text { Vouga }\end{array}$ & 27 & $\begin{array}{l}\text { BB, } \\
\text { CF, } \\
\text { GM, } \\
\text { ID, } \\
\text { W1, } \\
\text { W2 }\end{array}$ \\
\hline $\begin{array}{l}\text { Bravard_et } \\
\text { al_1999[55] }\end{array}$ & Europe & France & $\begin{array}{l}\text { River reaches (no } \\
\text { named dams) }\end{array}$ & $\begin{array}{l}\text { Rhone, } \\
\text { Drôme, } \\
\text { Drac, Ain }\end{array}$ & 6 & $\begin{array}{l}\text { CF, } \\
\text { GM, } \\
\text { W1 }\end{array}$ \\
\hline $\begin{array}{l}\text { Budhu_et_al } \\
\text { 1994[56] }\end{array}$ & N. America & $\begin{array}{l}\text { United } \\
\text { States }\end{array}$ & Glen Canyon Dam & Colorado & 5 & $\begin{array}{l}\text { GM, } \\
\text { W1 }\end{array}$ \\
\hline $\begin{array}{l}\text { Calles_et_al } \\
2013[57]\end{array}$ & Europe & Sweden & Ätrafors HEP & Atran & 6 & $\begin{array}{l}\mathrm{BB}, \\
\mathrm{W} 1\end{array}$ \\
\hline $\begin{array}{l}\text { Callisto_et } \\
\text { al_2005[58] }\end{array}$ & S. America & Brazil & $\begin{array}{l}\text { Paulo Afonso, Xingo, } \\
\text { and Moxoto Dams }\end{array}$ & $\begin{array}{l}\text { Sao } \\
\text { Francisco }\end{array}$ & 22 & $\begin{array}{l}\mathrm{BB}, \\
\mathrm{W} 1 \\
\mathrm{~W} 2\end{array}$ \\
\hline $\begin{array}{l}\text { Carley_et_al } \\
\text { _2012[59] }\end{array}$ & N. America & $\begin{array}{l}\text { United } \\
\text { States }\end{array}$ & $\begin{array}{l}\text { Daguerre Point and } \\
\text { Harry L. Englebright } \\
\text { Dams }\end{array}$ & Yuba & 12 & $\begin{array}{l}\text { GM, } \\
\text { W1 }\end{array}$ \\
\hline $\begin{array}{l}\text { Chicharo_et } \\
\text { al_2006 } \\
{[60]}\end{array}$ & Europe & Portugal & Alqueva Dam & Guadiana & 23 & $\begin{array}{l}\mathrm{BB}, \\
\mathrm{W} 1 \\
\mathrm{~W} 2\end{array}$ \\
\hline $\begin{array}{l}\text { Chiu_et_al_- } \\
2013[61]\end{array}$ & Asia & Taiwan & Unnamed dam & Dajia & 7 & $\begin{array}{l}\mathrm{BB}, \\
\mathrm{GM}\end{array}$ \\
\hline $\begin{array}{l}\text { Churchill_2 } \\
013 \text { [62] }\end{array}$ & N. America & $\begin{array}{l}\text { United } \\
\text { States }\end{array}$ & Denison Dam & Red & 21 & $\begin{array}{l}\mathrm{BB}, \\
\mathrm{W} 1 \\
\mathrm{~W} 2\end{array}$ \\
\hline $\begin{array}{l}\text { Craven_et_a } \\
1 \_2010[63]\end{array}$ & N. America & $\begin{array}{l}\text { United } \\
\text { States }\end{array}$ & $\begin{array}{l}\text { Flint River and R L } \\
\text { Harris Dams }\end{array}$ & $\begin{array}{l}\text { Flint, } \\
\text { Tallapoosa }\end{array}$ & 44 & $\begin{array}{l}\mathrm{BB}, \\
\mathrm{W} 1\end{array}$ \\
\hline $\begin{array}{l}\text { Dai_et_al_2 } \\
011[64]\end{array}$ & Asia & China & Three Gorges Dam & Yangtze & 12 & $\begin{array}{l}\text { W1, } \\
\text { W2 }\end{array}$ \\
\hline $\begin{array}{l}\text { Dauble_198 } \\
6[65]\end{array}$ & N. America & $\begin{array}{l}\text { United } \\
\text { States }\end{array}$ & Priest Rapids Dam & Columbia & 7 & $\begin{array}{l}\mathrm{BB}, \\
\mathrm{CF} \\
\mathrm{W} 2\end{array}$ \\
\hline
\end{tabular}




\begin{tabular}{|c|c|c|c|c|c|c|}
\hline $\begin{array}{l}\text { de_Almeida } \\
\text { etal_2005 } \\
\text { [66] }\end{array}$ & Europe & Portugal & $\begin{array}{l}\text { Fourteen hydropower } \\
\text { plants: Ribeiradio, Sr.a } \\
\text { do Monforte, Alvito, } \\
\text { Pêro Martins, Atalaia, } \\
\text { Asse-Dasse, Castelo } \\
\text { de Paiva, Castro Daire, } \\
\text { Póvoa, Midões, } \\
\text { Alvarenga, Pinhosão, } \\
\text { Portela, and } \\
\text { Girabolhos }\end{array}$ & $\begin{array}{l}\text { Vouga, } \\
\text { Côa, } \\
\text { Ocreza, } \\
\text { Mondego, } \\
\text { Paiva }\end{array}$ & 2 & W1 \\
\hline $\begin{array}{l}\text { deAlmeida_ } \\
\text { et_al_2003 } \\
\text { [67] }\end{array}$ & S. America & Brazil & $\begin{array}{l}\text { Barra Bonita, Ibitinga, } \\
\text { Mario Lopes Leao, } \\
\text { Tres Irmaos, } \\
\text { Taquarucu, Nova } \\
\text { Avanhadava, Rosana, } \\
\text { Capivara, and Bairi } \\
\text { Dams }\end{array}$ & $\begin{array}{l}\text { Tiete, } \\
\text { Paranapem } \\
\text { a }\end{array}$ & 3 & $\mathrm{BB}$ \\
\hline $\begin{array}{l}\text { Dean_and_S } \\
\text { chmidt_201 } \\
3 \text { [68] }\end{array}$ & N. America & $\begin{array}{l}\text { United } \\
\text { States }\end{array}$ & $\begin{array}{l}\text { Caballo, Elephant } \\
\text { Butte, Luis L. Leon, } \\
\text { La Boquilla, and } \\
\text { Francisco I. Madera } \\
\text { Dams }\end{array}$ & $\begin{array}{l}\text { Rio } \\
\text { Grande, } \\
\text { Rio } \\
\text { Conchos }\end{array}$ & 10 & $\begin{array}{l}\text { GM, } \\
\text { W1 }\end{array}$ \\
\hline $\begin{array}{l}\text { Duchemin_e } \\
\mathrm{t} \text { al_1995 } \\
{[69]^{-}}\end{array}$ & N. America & Canada & $\begin{array}{l}\text { Laforge } 1 \text { and La } \\
\text { Grande } 2 \text { Dams }\end{array}$ & $\begin{array}{l}\text { LaForge, } \\
\text { La Grande }\end{array}$ & 20 & $\begin{array}{l}\text { W1, } \\
\text { W2 }\end{array}$ \\
\hline $\begin{array}{l}\text { Ebel_1969 } \\
{[70]}\end{array}$ & N. America & $\begin{array}{l}\text { United } \\
\text { States }\end{array}$ & $\begin{array}{l}\text { Priest Rapids, } \\
\text { McNary, Ice Harbor, } \\
\text { Grand Coulee, } \\
\text { Bonneville, Chief } \\
\text { Joseph, The Dalles, } \\
\text { Wanupum, Rocky } \\
\text { Reach, and Rock } \\
\text { Island Dams }\end{array}$ & $\begin{array}{l}\text { Columbia, } \\
\text { Snake, }\end{array}$ & 18 & $\begin{array}{l}\text { BB, } \\
\text { CF, } \\
\text { W1, } \\
\text { W2 }\end{array}$ \\
\hline $\begin{array}{l}\text { Effler_et_al } \\
\text { _1988[71] }\end{array}$ & N. America & $\begin{array}{l}\text { United } \\
\text { States }\end{array}$ & Cannonsville Dam & Delaware & 25 & W2 \\
\hline $\begin{array}{l}\text { Englund_et } \\
\text { al_2008 [72] }\end{array}$ & Europe & Sweden & Mallengan & Lillian & 16 & $\begin{array}{l}\text { BB, } \\
\mathrm{LC}, \\
\mathrm{W} 2\end{array}$ \\
\hline $\begin{array}{l}\text { FosterAndR } \\
\text { ahs_1985 } \\
{[73]}\end{array}$ & N. America & Canada & Proposed dam & Stikine & 9 & BB \\
\hline $\begin{array}{l}\text { Frobrich_et } \\
\text { al_2007 } \\
{[74]}\end{array}$ & Asia & $\begin{array}{l}\text { Turkemeni } \\
\text { stan }\end{array}$ & Kaparas Dam & $\begin{array}{l}\text { Amu } \\
\text { Darya }\end{array}$ & 10 & $\begin{array}{l}\text { W1, } \\
\text { W2 }\end{array}$ \\
\hline $\begin{array}{l}\text { Gailbraith_a } \\
\text { nd_Vaughn } \\
2009[75]\end{array}$ & N. America & $\begin{array}{l}\text { United } \\
\text { States }\end{array}$ & $\begin{array}{l}\text { Pine Creek and Broken } \\
\text { Bow Dams }\end{array}$ & $\begin{array}{l}\text { Little, } \\
\text { Mountain } \\
\text { Fork }\end{array}$ & 13 & $\begin{array}{l}\mathrm{BB}, \\
\mathrm{W} 1\end{array}$ \\
\hline $\begin{array}{l}\text { Gain_et_al__ } \\
2013[76]\end{array}$ & Asia & Tibet & Zangmu Dam & $\begin{array}{l}\text { Brahmaput } \\
\text { ra }\end{array}$ & 28 & W1 \\
\hline
\end{tabular}




\begin{tabular}{|c|c|c|c|c|c|}
\hline $\begin{array}{l}\text { Galbraith_et } \\
\text { al_2015 } \\
{[77]}\end{array}$ & N. America & $\begin{array}{l}\text { United } \\
\text { States }\end{array}$ & $\begin{array}{l}\text { Parr Shoals, Upper } \\
\text { Androscoggin, } \\
\text { William O'Huske Lock } \\
\text { and Dam, New } \\
\text { Savannah Bluff Lock } \\
\text { and Dam, Adam T. } \\
\text { Bower Memorial } \\
\text { (inflatable) Dam, } \\
\text { Amoskeag, Falls } \\
\text { Village, Holts Pond } \\
\text { Dam, Holyoke, } \\
\text { Sinclair, Lloyd Shoals, } \\
\text { Tillery, Oxford, } \\
\text { Santee, Tar River } \\
\text { Reservoir Dam, John } \\
\text { H. Kerr }\end{array}$ & $\begin{array}{l}\text { Broad, } \\
\text { Androscog } \\
\text { gin, Cape } \\
\text { Fear, } \\
\text { Savannah, } \\
\text { Susquehan } \\
\text { na, } \\
\text { Merrimack } \\
\text { Housatonic } \\
\text {, Neuse, } \\
\text { Connecticu } \\
\text { t, Oconee, } \\
\text { Ocmulgee, } \\
\text { Pee Dee, } \\
\text { Catawba, } \\
\text { Santee, } \\
\text { Tar, } \\
\text { Roanoke }\end{array}$ & 9 \\
\hline $\begin{array}{l}\text { Gelwick_an } \\
\text { d_Mathews } \\
\text { _1990 [78] }\end{array}$ & N. America & $\begin{array}{l}\text { United } \\
\text { States }\end{array}$ & Denison Dam & Red River & 15 \\
\hline $\begin{array}{l}\text { Gobo_etAl_- } \\
2014 \text { [79] }\end{array}$ & Africa & Nigeria & Kainji Dam & Kainji & 5 \\
\hline $\begin{array}{l}\text { Graf_2006 } \\
{[80]}\end{array}$ & N. America & $\begin{array}{l}\text { United } \\
\text { States }\end{array}$ & $\begin{array}{l}\text { Albeni Falls, Beaver, } \\
\text { Blakely Mountain, } \\
\text { Buford, Center Hill, } \\
\text { Coolidge, Dnison, } \\
\text { Douglas, Eufaula, } \\
\text { Flaming Gorge, } \\
\text { Folsom, Fontana, } \\
\text { Grand Coulee, Greers } \\
\text { Ferry, Hartwell, } \\
\text { Hungry Horse, John H. } \\
\text { Kerr, Keystone, } \\
\text { Kinzua, Monticello, } \\
\text { Navajo, Norfork, } \\
\text { Norris, Oologah Lake, } \\
\text { Owyhee, Palisades, } \\
\text { Pine Flat, Sam } \\
\text { Rayburn, Sanford, } \\
\text { Sardis, Shasta, } \\
\text { Tenkiller Ferry, Tiber, } \\
\text { Tuttle Creek, Whitney, } \\
\text { and Wright Patman } \\
\text { Dams }\end{array}$ & $\begin{array}{l}\text { Allegheny, } \\
\text { American, } \\
\text { Angelina, } \\
\text { Arkansas, } \\
\text { Big Blue, } \\
\text { Brazos, } \\
\text { Canadian, } \\
\text { Caney } \\
\text { Fork, } \\
\text { Chattahooc } \\
\text { hee, } \\
\text { Clinch, } \\
\text { Columbia, } \\
\text { Flathead, } \\
\text { French } \\
\text { Broad, } \\
\text { Gila, } \\
\text { Green, } \\
\text { Illinois, } \\
\text { Kings, } \\
\text { Little Red, } \\
\text { Little } \\
\text { Tallahatchi } \\
\text { e, Little } \\
\text { Tennessee, } \\
\text { Marias, }\end{array}$ & 52 \\
\hline
\end{tabular}




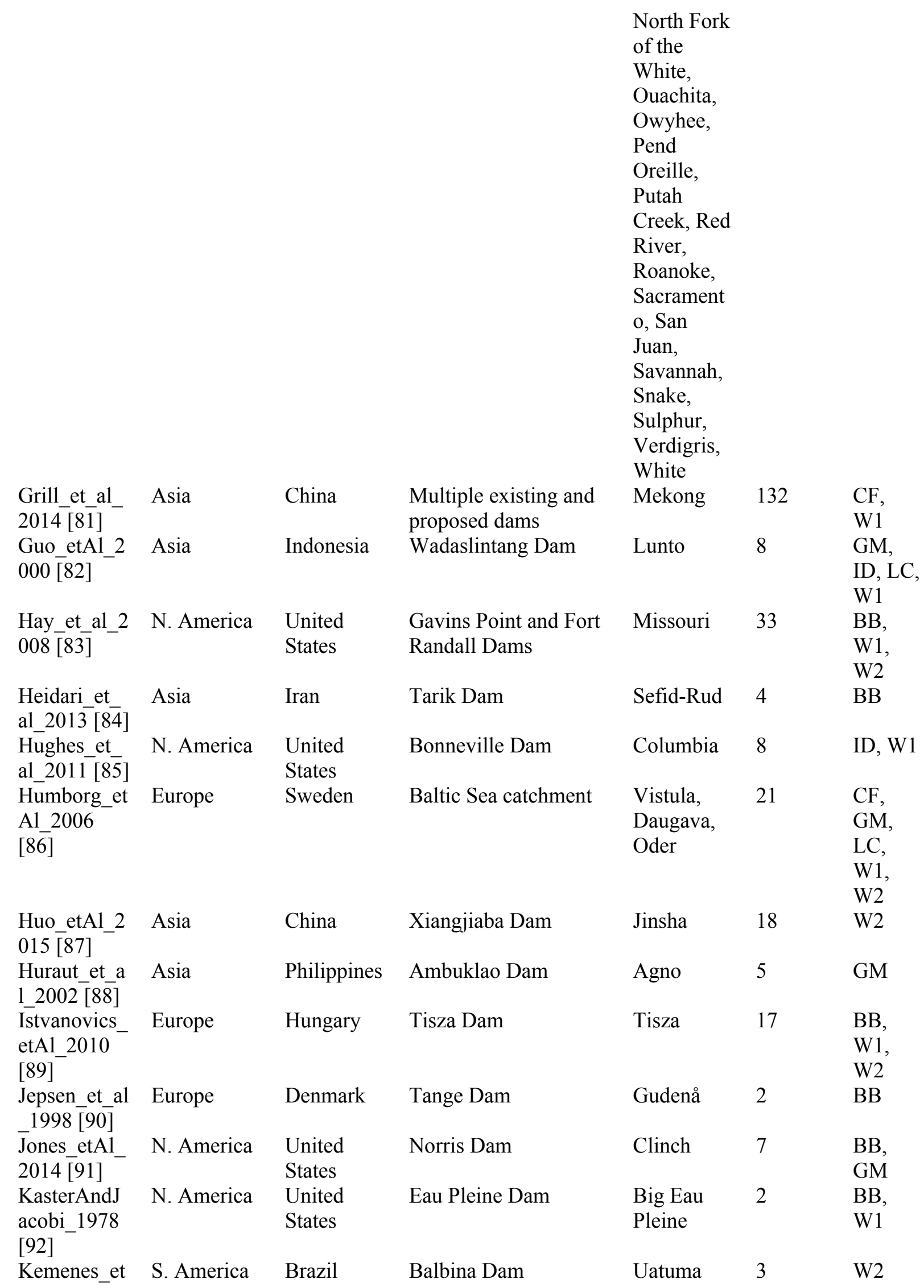




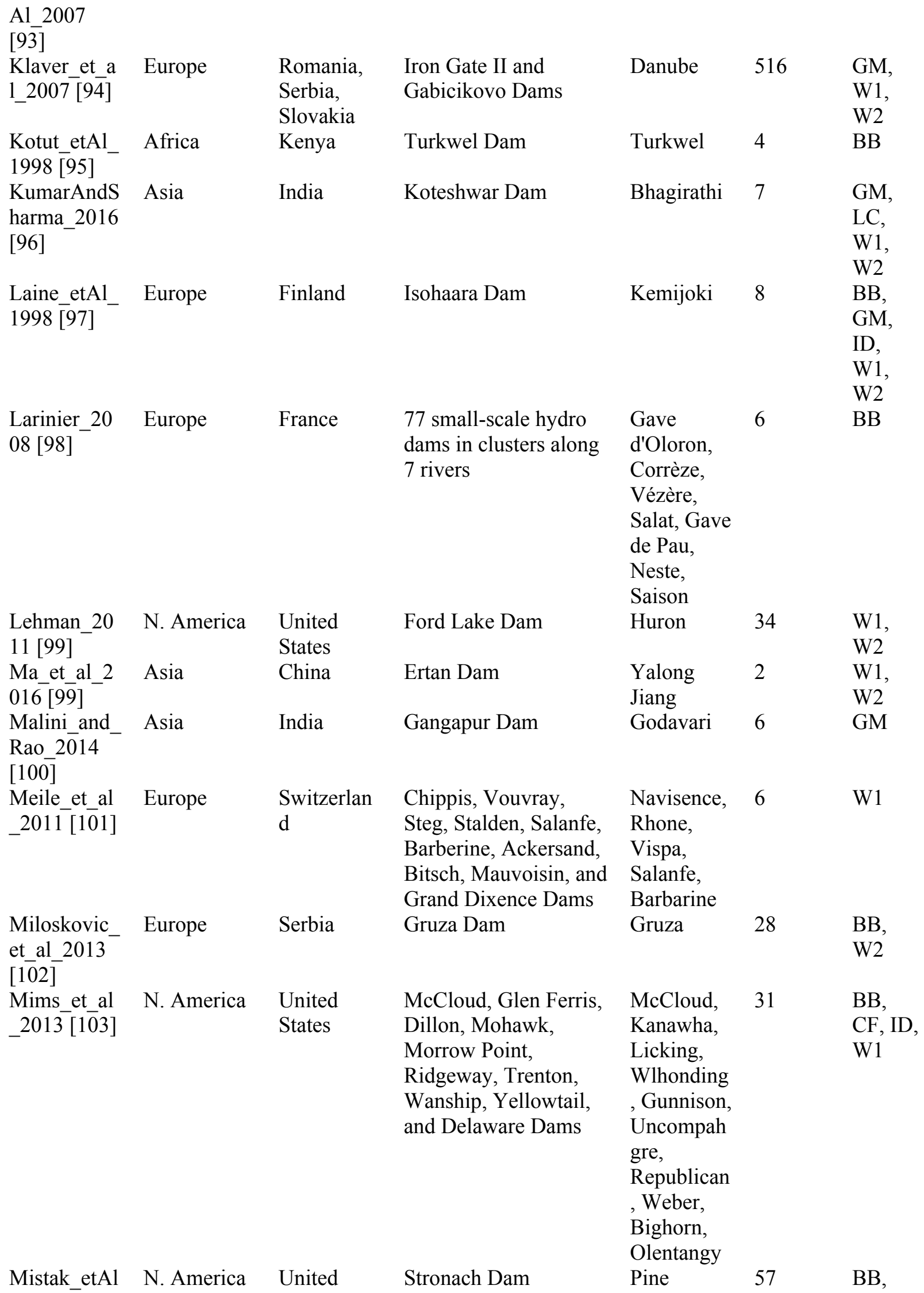




\begin{tabular}{|c|c|c|c|c|c|c|}
\hline 2003 [104] & & States & & & & $\begin{array}{l}\text { GM, } \\
\text { W2 }\end{array}$ \\
\hline $\begin{array}{l}\text { Muir_et_al_- } \\
2001[105]\end{array}$ & N. America & $\begin{array}{l}\text { United } \\
\text { States }\end{array}$ & $\begin{array}{l}\text { Lower Granite, Lower } \\
\text { Monumental, and } \\
\text { McNary Dams }\end{array}$ & $\begin{array}{l}\text { Snake, } \\
\text { Columbia }\end{array}$ & 7 & $\begin{array}{l}\mathrm{BB}, \\
\mathrm{W} 1\end{array}$ \\
\hline $\begin{array}{l}\text { Politano_et } \\
\text { Al_2012 } \\
{[106]}\end{array}$ & N. America & $\begin{array}{l}\text { United } \\
\text { States }\end{array}$ & Wells Dam & Columbia & 2 & $\mathrm{~W} 2$ \\
\hline $\begin{array}{l}\text { Ribi_etAl_2 } \\
014 \text { [107] }\end{array}$ & Europe & $\begin{array}{l}\text { Switzerlan } \\
\text { d }\end{array}$ & Maigrauge Dam & Sarine & 4 & $\begin{array}{l}\text { BB, ID, } \\
\text { W2 }\end{array}$ \\
\hline $\begin{array}{r}\text { Ribolli_etAl } \\
2012[108]\end{array}$ & S. America & Brazil & Machadinho Dam & Pelotas & 7 & BB \\
\hline $\begin{array}{l}\overline{\text { Scruton_etA }} \\
1 \_2005 \\
{[109]}\end{array}$ & N. America & Canada & West Salmon Dam & $\begin{array}{l}\text { West } \\
\text { Salmon }\end{array}$ & 8 & $\begin{array}{l}\mathrm{BB}, \\
\mathrm{GM}, \\
\mathrm{W} 1 \\
\mathrm{~W} 2\end{array}$ \\
\hline $\begin{array}{l}\text { Smith_etAl } \\
2016[110]\end{array}$ & N. America & Canada & E.B. Campbell Dam & $\begin{array}{l}\text { Saskatche } \\
\text { wan }\end{array}$ & 6 & GM \\
\hline $\begin{array}{l}\text { Soltani_et_a } \\
1 \_2010 \\
{[111]}\end{array}$ & Asia & Iran & 15-Khordad Dam & Ghomrud & 11 & $\begin{array}{l}\text { ID, LC, } \\
\text { W1, } \\
\text { W2 }\end{array}$ \\
\hline $\begin{array}{l}\text { Song_et_al_ } \\
2015[112]\end{array}$ & Asia & China & Three Gorges Dam & Yangtze & 8 & $\begin{array}{l}\text { GM, } \\
\text { W1 }\end{array}$ \\
\hline $\begin{array}{l}\text { Stevens_etA } \\
1 \_1995 \\
{[113]}\end{array}$ & N. America & $\begin{array}{l}\text { United } \\
\text { States }\end{array}$ & Glen Canyon Dam & Colorado & 7 & $\begin{array}{l}\mathrm{BB}, \\
\mathrm{GM}, \\
\mathrm{LC}\end{array}$ \\
\hline $\begin{array}{l}\text { Tamene_et_- } \\
\text { al_2006 } \\
{[114]}\end{array}$ & Africa & Ethiopia & $\begin{array}{l}\text { Group of micro dams } \\
\text { for supplemental } \\
\text { household irrigation. }\end{array}$ & $\begin{array}{l}\text { Tekeze } \\
\text { River } \\
\text { Basin }\end{array}$ & 18 & $\begin{array}{l}\text { GM, } \\
\text { ID, LC, } \\
\text { W1 }\end{array}$ \\
\hline $\begin{array}{l}\text { Thomaz_et } \\
\text { Al_2009 } \\
{[115]}\end{array}$ & S. America & Paraguay & Itaipu Dam & $\begin{array}{l}\text { Parana } \\
\text { Yacyreta }\end{array}$ & 7 & $\begin{array}{l}\mathrm{BB} \\
\mathrm{W} 2\end{array}$ \\
\hline $\begin{array}{l}\text { Thompson } \\
\text { etAl_2011 } \\
{[116]}\end{array}$ & N. America & $\begin{array}{l}\text { United } \\
\text { States }\end{array}$ & Camino Dam & $\begin{array}{l}\text { Silver } \\
\text { Creek }\end{array}$ & 13 & $\begin{array}{l}\mathrm{BB}, \\
\mathrm{GM}, \\
\mathrm{W} 1 \\
\mathrm{~W} 2\end{array}$ \\
\hline $\begin{array}{l}\text { Tufford_et_- } \\
\text { al_1999 } \\
\text { [117] }\end{array}$ & N. America & $\begin{array}{l}\text { United } \\
\text { States }\end{array}$ & Santee Dam & Santee & 37 & $\begin{array}{l}\text { BB, } \\
\text { W1, } \\
\text { W2 }\end{array}$ \\
\hline
\end{tabular}




\section{APPENDIX D. BIBLIOGRAPHY OF PEER-REVIEW LITERATURE THAT PASSED THE INITIAL REVIEW FILTER BUT WAS NOT INCLUDED IN THE DATABASE}

Appendix C. Papers in systematic review classified as relevant to environmental effects of hydropower and did not have metrics to be included in the metric database and catalog.As of 10 January 10, 2017, there were 148 studies that were reviewed but did not contain metrics.

Abell, R. (2002). Conservation biology for the biodiversity crisis: a freshwater follow-up. Conservation Biology, 16(5), 1435-1437.

Acaroglu, E. R., \& Graf, W. H. (1968). TRANSPORT IN CONVEYANCE SYSTEMS (Part 2) The modes of sediment transport and their related bed forms in conveyance.

Hydrological Sciences Journal, 13(3), 123-135.

Acua, P., Vila, I., \& Marn, V. H. (2008). Short-term responses of phytoplankton to nutrient enrichment and planktivorous fish predation in a temperate South American mesotrophic reservoir. Hydrobiologia, 600(1), 131-138.

Ĉada, G. F., \& Sale, M. J. (1993). Status of fish passage facilities at nonfederal hydropower projects. Fisheries, 18(7), 4-12.

Adams, S. M., Kimmel, B. L., \& Ploskey, G. R. (1983). Sources of organic matter for reservoir fish oroduction: a trophic-dynamics analysis. Canadian Journal of Fisheries and Aquatic Sciences, 40, 1480-1495.

Adhikary, P. (2014). Renovation modernization uprating $\backslash \&$ Llife extension : optimal solution for small hydropower development. International Journal of Engineering Science I\& Advanced Technology, 4(3), 300-306.

Adhikary, P., \& Kundu, S. (2014). Small Hydropower Project: Standard Practices. International Journal of Engineering Science \& Advanced Technology, 4(2), 241-247.

Afshar, A., \& Saadatpour, M. (2009). Reservoir Eutrophication Modeling, Sensitivity Analysis, and Assessment: Application to Karkheh Reservoir, Iran. Environmental Engineering Science, 26(7), 1227-1238.

Agrawal, D. K., \& Lodhi, M. S. (2010). Are EIA studies sufficient for projected hydropower development in the Indian Himalayan region? Current Science(Bangalore), 98(2), 154161.

Alemn-Nava, G. S., \& Casiano-Flores, V. H. (2014). Renewable energy research progress in Mexico: A review. Renewable and Sustainable Energy Reviews, 32, 140-153.

Allan, J. D. D. (2004). Landscapes and riverscapes: the influence of land use on stream ecosystems. Annual Review of Ecology, Evolution, and Systematics, 35(1), 257-284. 
Ansar, A. A., Flyvbjerg, B., Budzier, A., \& Lunn, D. (2014). Title : Should we build more large dams ? The actual costs of mega-dam development. Energy Policy, 69(1), 43--56.

Aman, N., \& Mawengkang, H. (2014). A Sustainable Forest Management Optimization Model to Preserve Water Allocation for Hydroelectric Power Plant. International Journal of Science and Advanced Technology, 4(1), 5--9.

Avoaja, D. A., Inyang, N. M., \& Mgbenka, B. O. (2007). Macrobenthic fauna of a humid tropical water reservoir, Abia State, Nigeria. Animal Research International, 4(2), 677-679.

Babbitt, B. (2002). What Goes Up, May Come Down Learning from our experiences with dam construction in the past can guide and improve dam removal in the future. BioScience, 52(8), 656-658.

Bailey, R. G., \& Churchfield, S. (1978). Observations on the zooplankton and littoral macroinvertebrates of Nyumba ya Mungu reservoir, Tanzania. Biological Journal of the, $10(1), 93-107$.

Bakis, R., \& Demirbas, A. (2004). Sustainable development of small hydropower plants (SHPs). Energy Sources, 26(2), 1105-1118.

Ballabh, H., Pillay, S., \& Negi, G. C. S. (2014). Relationship between Selected Physiographic Features and Landslide Occurrence around Four Hydropower Projects in Bhagirathi Valley of Uttarakhand, Western. International Journal of Geosciences, 5(10), 1088-1099.

Barbieri, R., \& Esteves, F. d. A. (1991). The chemical composition of some aquatic macrophyte species and implications for the metabolism of a tropical lacustrine ecosystem-Lobo Reservoir, Sao Paulo. Hydrobiologia, 213(2), 133-140.

Barthem, R. B., Ribeiro, M. C. L. d. B., \& Petrere, M. (1991). Life strategies of some longdistance migratory catfish in relation to hydroelectric dams in the Amazon Basin. Biological Conservation, 553(3), 339-345.

Bednarek, A. T. (2001). Undamming rivers: a review of the ecological impacts of dam removal. Environmental Management, 27(6), 803-814.

Benko, K. L., \& Drewes, J. E. (2008). Produced water in the Western United States: geographical distribution, occurrence, and composition. Environmental Engineering Science, 25(2).

Bianco, P. G., \& Ketmaier, V. (2015). Nature and status of freshwater and estuarine fisheries in Italy and Western Balkans. Freshwater Fisheries Ecology, 283-291.

Bicudo, D. D. C., Fonseca, B. M., Bini, L. M., Crossetti, L. O., Bicudo, C. E., \& Araujo-Jesus, T.A.T.I.A.N.E. (2007). Undesirable side-effects of water hyacinth control in a shallow tropical reservoir. Freshwater Biology, 52(6), 1120-1133. 
Bielańska-Grajner, I., \& Poznańska, M. (2010). The biodiversity of psammon rotifers in Włocławek Reservoir. Oceanological and Hydrobiological Studies, 39(1), 111-117.

Bolger, T., Bracken, J. J., \& Dauod, H. A. (1990). The feeding relationships of brown trout, minnow and three-spined stickleback in an upland reservoir system. Hydrobiologia, 208(3), 169-185.

Born, S. M., Genskow, K. D., \& Filbert, T. L. (1998). Socioeconomic and institutional dimensions of dam removals: the Wisconsin experience. Environmental Management, 22(3), 359-370.

Boschilia, S. M., \& de Oliveira, E. F. (2012). The immediate and long-term effects of water drawdown on macrophyte assemblages in a large subtropical reservoir. Freshwater Biology, 57(12), 2641-2651.

Bosnia, A. S., Kaisin, F. J., \& Tablado, A. (1990). Population dynamics and production of the freshwater snail Chilina gibbosa Sowerby 1841 (Chilinidae, Pulmonata) in a NorthPatagonian reservoir. Hydrobiologia, 190(2), 97-110.

Bouvy, M., Nascimento, S. M., Molica, R. J. R., \& Ferreira, A. (2003). Limnological features in Tapacura reservoir (northeast Brazil) during a severe drought. Hydrobiologia, 493(1), $115-130$.

Brdossy, A., Wagner, S., Kunstmann, H., \& Brdossy, A. (2006). Model based distributed water balance monitoring of the White Volta catchment in West Africa through coupled meteorological-hydrological simulations. Advances in Geosciences, 9, 39-44.

Bremigan, M. T., \& Stein, R. A. (2001). Variable gizzard shad recruitment with reservoir productivity: causes and implications for classifying systems. Ecological Applications, $11(5), 1425-1437$.

Briscoe, J. (1999). The financing of hydropower, irrigation and water supply infrastructure in developing countries. International Journal of Water Resources Development, 15(4), $459-491$.

Brown, P. H., Magee, D., \& Yilin, X. U. (2008). Socioeconomic vulnerability in China's hydropower development. China Economic Review, 19(4), 614-627.

Buhe, A., Tsuchiya, K., Kaneko, M., \& Ohtaishi, N. (2007). Land cover of oases and forest in XinJiang, China retrieved from ASTER data. Advances in Space, 39(1), 39-45.

Bunn, S. E., \& Arthington, a. H. (2002). Basic Principles and Ecological Consequences of Altered Flow Regimes for Aqatic Biodiversity (0026700227370

Carr, G. M. (1998). Macrophyte growth and sediment phosphorus and nitrogen in a Canadian prairie river. Freshwater Biology, 39(3), 525-536. 
Carr, G. M., Duthie, H. C., \& Taylor, W. D. (1997). Models of aquatic plant productivity: a review of the factors that influence growth. Aquatic Botany, 59(3-4), 195-215.

Carrasco, L., Benejam, L., Benito, J., \& Bayona, J. M. (2011). Methylmercury levels and bioaccumulation in the aquatic food web of a highly mercury-contaminated reservoir. Environment, 37(7), 1213-1218.

Cavendish, M. G. M. G., \& Duncan, M. I. M. I. (1986). Use of the Instream Flow Incremental Methodology : a Tool for Negotiation. Environmental Impact Assessment Review, 6(4), 347-363.

Chandel, S. S., Shrivastva, R., \& Sharma, V. (2016). Overview of the initiatives in renewable energy sector under the national action plan on climate change in India. Renewable and Sustainable Energy Reviews, 54, 866-873.

Cho, H. J., \& Jang, C. L. (2014). Numerical Simulation for Estimating Fish Shelter at the Downstream of Gumi Weir. Ecology and Resilient Infrastructure, 1(1), 8-18.

Choubey, V. K. (1998). Laboratory experiment, field and remotely sensed data analysis for the assessment of suspended solids concentration and secchi depth of the reservoir surface water. International Journal of Remote Sensing, 19(17), 3349-3360.

Chu, Q. H., Li, W. H., \& Sun, C. H. (2012). Research of Economic Operation in Hydropower Station Based on Recursive Optimization. 524-527, 2905-2910.

Chu, Y. S., Cho, H., \& Cho, K. H. (2016). Response of Vegetation to Shoreline Alternation in a Large Reservoir. Ecology and Resilient Infrastructure, 3(2), 143-151.

Clifford, H. F., Wiley, G. M., \& Casey, R. J. (1993). Macroinvertebrates of a beaver-altered boreal stream of Alberta, Canada, with special reference to the fauna on the dams. Canadian Journal of Zoology, 71(7), 1439-1447.

Clifford, N. J., Harmar, O. P., \& Harvey, G. (2006). Physical habitat, eco-hydraulics and river design: a review and re-evaluation of some popular concepts and methods. Aquatic Conservation: Marine and Freshwater Ecosystems, 16(4), 189-408.

Cochrane, K. L. (1987). The biomass and yield of the dominant fish species in Hartbeespoort Dam, South Africa. Hydrobiologia, 146(1), 89-96.

Colby, J. D., \& Keating, P. L. (1998). Land cover classification using Landsat TM imagery in the tropical highlands: the influence of anisotropic reflectance. International Journal of Remote Sensing, 19(8), 1479-1500.

Colotelo, A. H., Pflugrath, B. D., Brown, R. S., \& Brauner, C. J. (2012). The effect of rapid and sustained decompression on barotrauma in juvenile brook lamprey and Pacific lamprey: Implications for passage at hydroelectric facilities. Fisheries, 129-130, 17-20. 
Conner, W., Barton, C., Nelson, E. A., Kolka, R. K., McLeod, K. W., Lakly, M., Martin, D., Wiggington J., Trettin, C., \& Wisniewski, J. (2000). Restoration of a severely impacted riparian wetland system-The Pen Branch Project. Ecological Engineering.

Crawford, K., Allen, D., \& Aharon, A. (2009). Irrigation Research: Making Connections about Connectivity. Irrigation Australia: The Official Journal of Irrigation Australia, 24(3), 25.

Crossetti, L. O., Bicudo, D. C., \& Bicudo, C. E. M. (2008). Phytoplankton biodiversity changes in a shallow tropical reservoir during the hypertrophication process. Brazilian Journal of Biology, 6(4), 1061-1067.

Ḉullaj, A., Duka, S., Emiri, A., Koni, E., \& Miho, A. (2011). Limnological study on a newly built drinking water reservoir near Tirana, Albania. Environmental Monitoring and Assessment, 182(1), 215-232.

Curtarelli, M. P., Alcântara, E. H., Rennó, C. D., \& Stech, J. L. (2013). Modeling the effects of cold front passages on the heat fluxes and thermal structure of a tropical hydroelectric reservoir. Hydrology \& Earth System Sciences Discussions, 10(7).

Daldorph, P. W. G. (1999). A reservoir in management-induced transition between ecological states. Hydrobiologia, 395(0), 325-333.

Dam, R. A. V., Camilleri, C., \& Finlayson, C. M. (1998). The potential of rapid assessment techniques as early warning indicators of wetland degradation: A review. Environmental Toxicology and Water Quality, 13(4), 297-312.

Darrigran, G. (2002). Potential impact of filter-feeding invaders on temperate inland freshwater environments. Biological Invasions, 4(1), 1145-1156.

Davies, B. R., Hall, A., \& Jackson, P. B. N. (1975). Some ecological aspects of the Cabora Bassa dam. Biological Conservation, 8(3), 189-201.

Davoren, A., \& Mosley, M. P. (1986). Observations of bedload movement, bar development and sediment supply in the braided Ohau River. Earth Surface Processes and, 11(6), 643-652.

de la Croix, J. F. (2011). Moving metaphors we live by: water and flow in the social sciences and around hydroelectric dams in Kyrgyzstan. Central Asian Survey, 30(3-4), 487-502.

del Nordesie, I. D. I. (2000). Fish community variation below Yacyretá Dam (Paraná River, Argentina): the relative contribution of microhabitat, hydrology and limnology. Acta Llmnologica Bias, 12(2338), 23.

Demars, B. O. L., Potts, J. M., \& Tremolieres, M. (2012). River macrophyte indices: not the Holy Grail! Freshwater, 57(8), 1745-1759. 
Demirbaş, A. (2002). Sustainable developments of hydropower energy in Turkey. Energy Sources, 24(1), 27-40.

Denny, P., \& Bowker, D. W. (1978). The importance of the littoral epiphyton as food for commercial fish in the recent African man-made lake, Nyumba ya Mungu reservoir, Tanzania. Biological Journal of the, 10(1), 139-150.

Dhar, A., \& Datta, B. (2008). Optimal operation of reservoirs for downstream water quality control using linked simulation optimization. Hydrological Processes, 22(6), 842-853.

Diomanda, D., Kpai, N. N., \& Kouadip, K. N. (2013). Spatial distribution and structure of benthic macroinvertebrates in an artificial reservoir: Taabo Lake (Côte d'Ivoire). International Journal of, 17(4), 1503-1514.

Domingues, R. B., \& Galvao, H. (2007). Phytoplankton and environmental variability in a dam regulated temperate estuary. Hydrobiologia, 586(1), 117-134.

Doyle, M. W., Harbor, J. M., \& Stanley, E. H. (2003). Toward policies and decision-making for dam removal. Environmental Management, 31(4), 0453-0465.

Doyle, M. W., Stanley, E. H., Orr, C. H., Selle, A. R., \& Sethi, S. A. (2005). Stream ecosystem response to small dam removal: lessons from the Heartland. Geomorphology, 71(1-2), 227-244.

Dudgeon, D. (2000). Riverine biodiversity in Asia: a challenge for conservation biology. Hydrobiologia, 418(1), 1-13.

Egr. (2002). The diversity of hydropower projects. Energy Policy, 30(14), 1225-1230.

Falkenmark, M., \& Folke, C. (2002). The ethics of socio-ecohydrological catchment management: towards hydrosolidarity. Hydrology and Earth System, 6(1), 1-10.

Fang, Y., \& Deng, W. (2011). The critical scale and section management of cascade hydropower exploitation in Southwestern China. Energy, 36(10), 5944-5953.

Fleischer, T. (1993). Jaws on the Danube: water management, regime change and the movement against the Middle Danube Hydroelectric Dam. International Journal of Urban and Regional, 17(3), 429-443.

France, R. L. (1997). Macroinvertebrate colonization of woody debris in Canadian Shield lakes following riparian clearcutting. Conservation Biology, 11(2), 513-521.

Franklin, P. A., \& Bartels, B. (2012). Restoring connectivity for migratory native fish in a New Zealand stream: effectiveness of retrofitting a pipe culvert. Aquatic Conservation: Marine and, 22(4), 489-497. 
Freitas, C. E. C., \& Petrere, M. (2005). Natural and artificially-induced habitat complexity and freshwater fish species composition. Fisheries Management and, 12(1), 63-67.

Fron, P., Cury, P., \& Shannon, L. (2005). Sustainable exploitation of small pelagic fish stocks challenged by environmental and ecosystem changes: a review. Bulletin of Marine Science, 76(2), 385-462.

Gahalaut, K., \& Gahalaut, V. K. (2010). Effect of the Zipingpu reservoir impoundment on the occurrence of the 2008 Wenchuan earthquake and local seismicity. Geophysical Journal International, 183(1), 277--285. doi:10.1111/j.1365-246X.2010.04715.x

Ghrefat, H., \& Yusuf, N. (2006). Assessing Mn, Fe, Cu, Zn, and Cd pollution in bottom sediments of Wadi Al-Arab Dam, Jordan. Chemosphere, 65(11), 2114--2121.

Gillespie, B. R., Desmet, S., Kay, P., Tillotson, M. R., \& Brown, L. E. (2015). A critical analysis of regulated river ecosystem responses to managed environmental flows from reservoirs. Freshwater Biology, 60(2), 410-425.

Gogoi, T. R. (2016). The Golden River Hydro Electric Power Project and its impacts on Downstream. Imperial Journal of Interdisciplinary Research, 2(9).

Goodwin, R. A., Nestler, J. M., Anderson, J. J., \& Weber, L. J. (2006). Forecasting 3-D fish movement behavior using a Eulerian-Lagrangian-agent method (ELAM). Ecological, 192(1-2), 197--223.

Goretti, E., Ceccagnoli, D., \& Giovanni, M. V. D. (2006). Reservoir management and its effects on the trophic organization of the macrobenthic community in a river in central Italy. Italian Journal of Zoology, 73(1), 33-41.

Graf, W. L. (2005). Geomorphology and American dams: the scientific, social, and economic context. Geomorphology, 71(1-2), 3-26.

Grafton, R. Q., \& Kompas, T. (2007). Pricing sydney water. Australian Journal of Agricultural and Resource Economics, 51(3), 227-241.

Grant, G. E. (2001). Dam removal: Panacea or Pandora for rivers? Hydrological Processes, 15(8), 1531--1532. doi:10.1002/hyp.473

Griffin, J. B. (1971). Archeological Investigations in the Grand Rapids, Manitoba, Resevoir 1961-62, by William J. Mayer-Oakes. Arctic, 24(4), 309-310.

Ha, K., Jang, M. H., \& Joo, G. J. (2003). Winter Stephanodiscus bloom development in the Nakdong River regulated by an estuary dam and tributaries. Hydrobiologia, 506(1), 221227. 
Harrison, G. P., Whittington, H. W., \& Wallace, A. R. (2006). Sensitivity of hydropower performance to climate change. International journal of power \& energy systems, 26(1), 42.

Hart, R. C. (1987). Population dynamics and production of five crustacean zooplankters in a subtropical reservoir during years of contrasting turbidity. Freshwater Biology, 18(2), 287-318.

Harvey, R. M., Pickett, J. R., \& Bates, R. D. (1987). Environmental factors controlling the growth and distribution of submersed aquatic macrophytes in two South Carolina reservoirs. Lake and Reservoir Management, 3(1), 243-255.

Hayakawa, Y. S., \& Matsukura, Y. (2009). Factors influencing the recession rate of Niagara Falls since the 19th century. Geomorphology, 110(3-4), 212-216.

Heiskary, S. A., Wilson, C. B., \& Larsen, D. P. (1987). Analysis of regional patterns in lake water quality: Using ecoregions for lake management in Minnesota. and Reservoir Management, 3(1), 337-344.

Heitfeld, K. H., \& Krapp, L. (1981). The problem of water permeability in dam geology. Bulletin of the International Association of Engineering Geology-Bulletin de l'Association Internationale de Géologie de l'Ingénieur, 23(1), 79-83.

Hocking, G. C., \& Patterson, J. C. (1994). Modelling tracer dispersal and residence time in a reservoir. Ecological Modelling, 74(1-2), 63-75.

Idowu, R. T., Inyang, N. M., \& Ezenwaji, H. M. (2004). Heavy Metal Concentrations In A West African Sahel Reservoir. Animal Research International, 1(1), 12-15.

Igliski, B., Igliska, A., Koziski, G., \& Skrzatek, M. (2016). Wind energy in Poland-History, current state, surveys, Renewable Energy Sources Act, SWOT analysis. Renewable and Sustainable Energy Reviews, 64, 19-33.

Ikebuchi, S., \& Kojiri, T. (1992). Multi-Objective Reservoir Operation Including Turbidity Control1. JAWRA Journal of the American Water Resources Association, 28(1), 223-231.

Johnson, R. L., \& Simmons, M. A. (2001). A new multibeam sonar technique for evaluating fine-scale fish behavior near hydroelectric dam guidance structures. 161-170.

Júnior, I. B., \& da Cunha-Santino, M. B. (2014). Dynamics of colonization and the collapse of a macrophyte community during the formation of a tropical reservoir. Fundamental and Applied Limnology/Archiv für Hydrobiologie, 184(2), 141-150.

Kelly, M. S. (1989). Distribution and biomass of aquatic macrophytes in an abandoned nuclear cooling reservoir. Aquatic Botany, 35(2), 133--152. 
Kikuchi, R., \& do Amaral, P. B. (2008). Conceptual schematic for capture of biomethane released from hydroelectric power facilities. Bioresource technology, 99(13), 5967-5971.

King, S. L., Allen, J. A., \& McCoy, J. W. (1998). Long-term effects of a lock and dam and greentree reservoir management on a bottomland hardwood forest. Forest Ecology and Management, 112(3), 213-226.

Lanta, V., Mach, J., \& Holcov. (2006). The effect of dam construction on the restoration succession of spruce mires in the Giant Mountains (Czech Republic). Annales botanici fennici, 43(4), 260-268.

Li, X., Wei, X., \& Wei, N. (2016). Correlating check dam sedimentation and rainstorm characteristics on the Loess Plateau, China. Geomorphology, 265, 84-97.

Limburg, K. E., \& Hattala, K. A. (2003). American shad in its native range. Biodiversity, Status, and Conservation of the World's Shads, 125--140.

Liu, B., Yan, H., Wang, C., Li, Q., Guédron, S., Spangenberg, J. E., ... \& Dominik, J. (2012). Insights into low fish mercury bioaccumulation in a mercury-contaminated reservoir, Guizhou, China. Environmental pollution, 160, 109-117.

Magilligan, F. J. (1992). Thresholds and the spatial variability of flood power during extreme floods. Geomorphology, 5(3-5), 373-390.

Magoulick, D. D., \& Lewis, L. C. (2002). Predation on exotic zebra mussels by native fishes: effects on predator and prey. Freshwater Biology, 47(10), 1908-1918.

Mark, A. F., \& Johnson, P. N. (1985). Ecologically derived guidelines for managing two New Zealand lakes. Environmental Management, 9(4), 355--363.

Martel, P., Kovacs, T., Voss, R., \& Megraw, S. (2003). Evaluation of caged freshwater mussels as an alternative method for environmental effects monitoring (EEM) studies. Environmental Pollution, 124(3), 471-483.

Martin-Ortega, J., Ojea, E., \& Roux, C. (2013). Payments for water ecosystem services in Latin America: a literature review and conceptual model. Ecosystem Services, 6, 122-132.

Marzin, A., Archaimbault, V., Belliard, J., Chauvin, C., Delmas, F., \& Pont, D. (2012). Ecological assessment of running waters: do macrophytes, macroinvertebrates, diatoms and fish show similar responses to human pressures?. Ecological indicators, 23, 56-65.

McCullough, D. A. (2010). Are coldwater fish populations of the United States actually being protected by temperature standards? Freshwater reviews, 3(2), 147--199. 
Merritt, D. M., Scott, M. L., LeRoy, P., \& Auble, G. T. (2010). Theory, methods and tools for determining environmental flows for riparian vegetation: riparian vegetation-flow response guilds. Freshwater, 55(1), 206--225.

Moser, M. L., Zabel, R. W., Burke, B. J., Stuehrenberg, L. C., \& Bjornn, T. C. (2005). Factors affecting adult Pacific lamprey passage rates at hydropower dams: using "time to event" analysis of radiotelemetry data. Aquatic telemetry: advances and applications. Food and Agriculture Organization of the United Nations and COISPA TECONOLOGIA \& RICERCA, Rome, 61-70.

Neely, B. C., Pracheil, B. M., \& Lynott, S. T. (2014). Influence of river discharge on recreational harvest of paddlefish. Fisheries Management and Ecology, 21(4), 259-263. d

Novikmec, M., \& Svitok, M. (2003). Influence of the operation of small hydropower station on the communities of selected groups of macrozoobenthos (Ephemeroptera, Plecoptera, Trichoptera) of the submountain section of Hucava river (Polana Mts., Slovakia)preliminary results. Acta Facultatis Ecologiae, 10(suppl. 1), 175-178.

Otahelová, H., \& Valachovic, M. (2003). Distribution of macrophytes in different water-bodies (habitats) influenced by the Gabčíkovo hydropower station (Slovakia): present status. Archiv für Hydrobiologie. Supplementband. Large rivers, 14(1-2), 97-115.

Palus, J., Dziubatowska, E., \& Staczyk, M. (2007). Biomonitoring of cyanobacterial blooms in Polish water reservoir and the cytotoxicity and genotoxicity of selected cyanobacterial extracts. International Journal of Occupational Medicine and Environmental Health, $20(1)$.

Parkinson, S. C., \& Djilali, N. (2015). Robust response to hydro-climatic change in electricity generation planning. Climatic Change, 130(4), 475-489.

Pegg, M. A., \& Pierce, C. L. (2002). Fish community structure in the Missouri and lower Yellowstone rivers in relation to flow characteristics. Hydrobiologia, 479(1), 155-167.

Petticrew, E. L., Krein, A., \& Walling, D. E. (2007). Evaluating fine sediment mobilization and storage in a gravel-bed river using controlled reservoir releases. Hydrological Processes, 21(2), 198-210.

Piegay, H., Thevenet, A., \& Kondolf, G. M. (2000). Physical and human factors influencing potential fish habitat distribution along a Mountain River, France. Annaler: Series A, ldots, 82(1), 121--136.

Poff, N. L. R., Bledsoe, B. P., \& Cuhaciyan, C. O. (2006). Hydrologic variation with land use across the contiguous United States: geomorphic and ecological consequences for stream ecosystems. Geomorphology, 79(3-4), 264-285. 
Pringle, C. (2003). What is hydrologic connectivity and why is it ecologically important? Hydrological Processes, 17(13), 2685--2689. doi:10.1002/hyp.5145

Pringle, C. M., Freeman, M. C., \& Freeman, B. J. (2000). Regional effects of hydrologic alterations on riverine macrobiota in the new world: tropical-temperate comparisons . BioScience, 50(9), 807--823.

Reddy, M. J., \& Kumar, D. N. (2008). Evolving strategies for crop planning and operation of irrigation reservoir system using multi-objective differential evolution. Irrigation Science, 26(2), 177--190.

Rehmann, C. R., \& Stoeckel, J. A. (2003). Effect of turbulence on the mortality of zebra mussel veligers. Canadian Journal of, 81(6), 1063-1069.

Richmond, M. C., Serkowski, J. A., Ebner, L. L., \& Sick, M. (2014). Quantifying barotrauma risk to juvenile fish during hydro-turbine passage. Fisheries, 154, 152--164.

Salafsky, N., Salzer, D., \& Stattersfield, A. J. (2008). A standard lexicon for biodiversity conservation: unified classifications of threats and actions. Conservation, 22(4), 897-911.

Santi, P. M., Ey, J., \& Gartner, J. E. (2008). Effective mitigation of debris flows at Lemon Dam, La Plata County, Colorado. Geomorphology, 96(3-4), 366--377.

Sanu, P., \& Newport, J. K. (2010). Invasive alien species dispersal: the millennium biodiversity disaster. Disaster Prevention and Management:, 19(3), 291-297.

Schwarz, U., Bloesch, J., Chen, Y., \& Takara, K. (2004). GIS-supported mitigation of the impact of hydropower dams on the flood plains of the Drava-Mura Rivers in Croatia/Hungary. 178--187.

Shrestha, H. S. (2012). Application of Hydrosuction Sediment Removal System (HSRS) on Peaking Ponds. Hydro Nepal: Journal of Water, Energy and Environment, 11, 43-48.

Shukla, S. K., \& Pandey, D. N. (2012). Envrionmental restoration around the Rihand Dam. International Journal of Science and Research Publications, 2(11), 1-7.

Sissakian, V. K., Al-Ansari, N., \& Knutsson, S. (2014). Karstification effect on the stability of Mosul Dam and its assessment, North Iraq. Engineering, 6(2).

Smakhtin, V. (2008). Basin Closure and Environmental Flow Requirements. International Journal of Water Resources Development, 24(2), 227-233.

Soncini-Sessa, R., Villa, L., \& Weber, E. (1999). TwoLe: a software tool for planning and management of water reservoir networks. Hydrological Sciences, 44(4), 619-631. 
Stovin, V. R., Jorgensen, A., \& Clayden, A. (2008). Street trees and stormwater management. Arboricultural Journal, 30(4), 297--310.

Sulis, A., Buscarinu, P., \& Sechi, G. M. (2011). Using reservoir trophic-state indexes in optimisation modelling of water-resource systems. Environmental Modelling \& Software, 26(6), 731-738.

Tanaka, S. K., Zhu, T., Lund, J. R., Howitt, R. E., \& Jenkins, M. W. (2006). Climate warming and water management adaptation for California. Climatic Change, 76(3), 361--387.

Tarkan, A. S., \& Gaygusuz. (2012). Circumstantial evidence of gibel carp, Carassius gibelio, reproductive competition exerted on native fish species in a mesotrophic reservoir. Fisheries, 19(2), 167-177.

Taylor, C. M., Millican, D. S., Roberts, M. E., \& Slack, W. T. (2008). Long-term change to fish assemblages and the flow regime in a southeastern US river system after extensive aquatic ecosystem fragmentation. Ecography, 31(6), 787-797.

Truffer, B., Bratrich, C., Markard, J., Peter, A., West, A., Wuest, a., \& Wehrli, B. (2003). Green Hydropower: The contribution of aquatic science research to the promotion of sustainable electricity. Aquatic Sciences, 65(2), 99--110.

Zolotov, L. A., Karelin, V. Y., \& Stroganov, E. M. (1973). Current trends in the design, investigation, and operation of hydraulic machines for pumped-storage plants. Hydrotechnical Construction, 7(8), 784-787. 